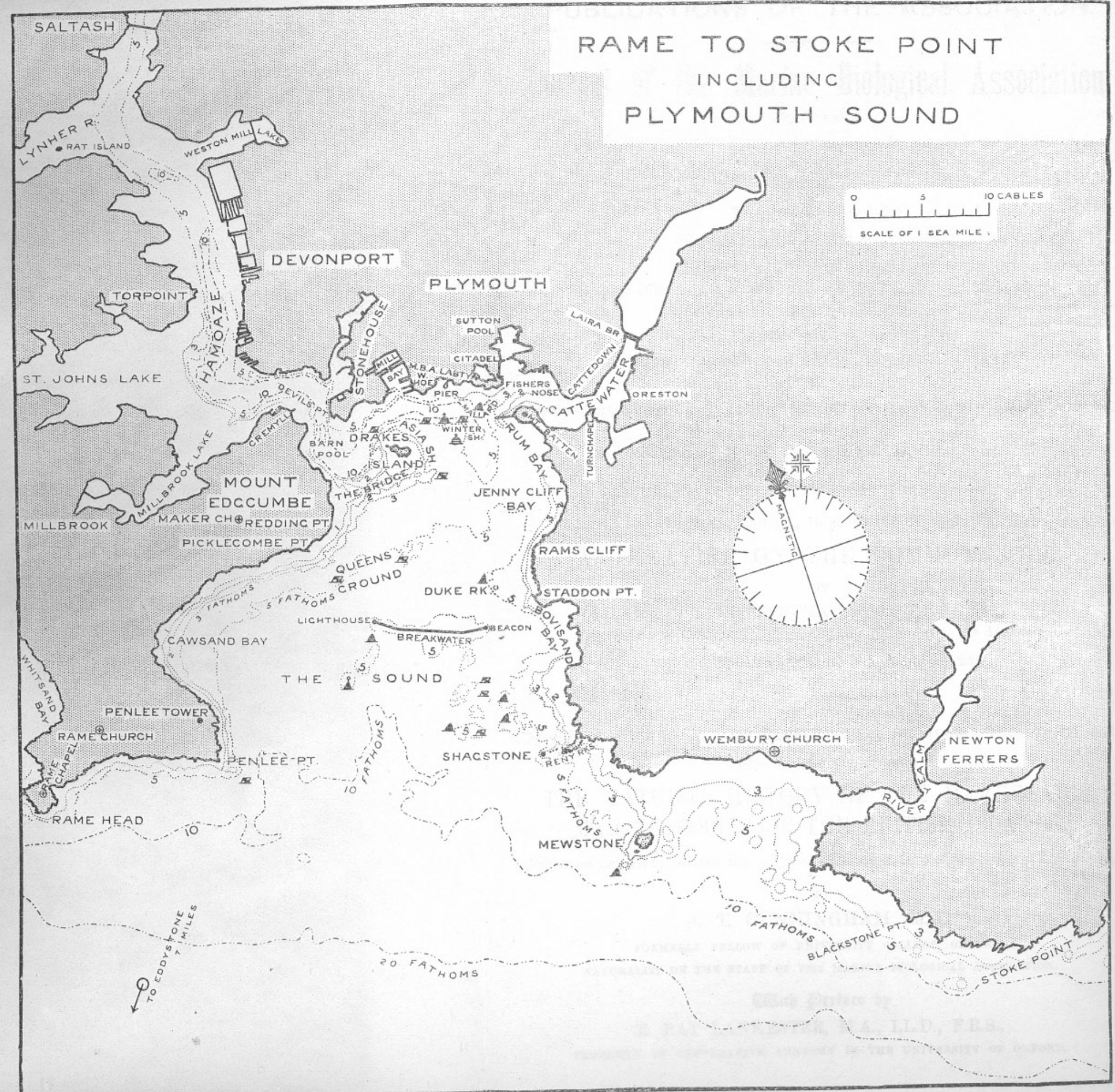

Jour. Mar. Biol, Assoc. Vol, vii, 


\title{
Plymouth Marine Invertebrate Fauna
}

\author{
Being Notes of the Local Distribution of Species occurring in the \\ Neighbourhood.
}

\author{
COMPILED FROM THE RECORDS OF THE LABORATORY \\ OF THE
}

\section{Marine Biological Association.*}

(With one Chart.)

\section{TABLE OF CONTENTS.}

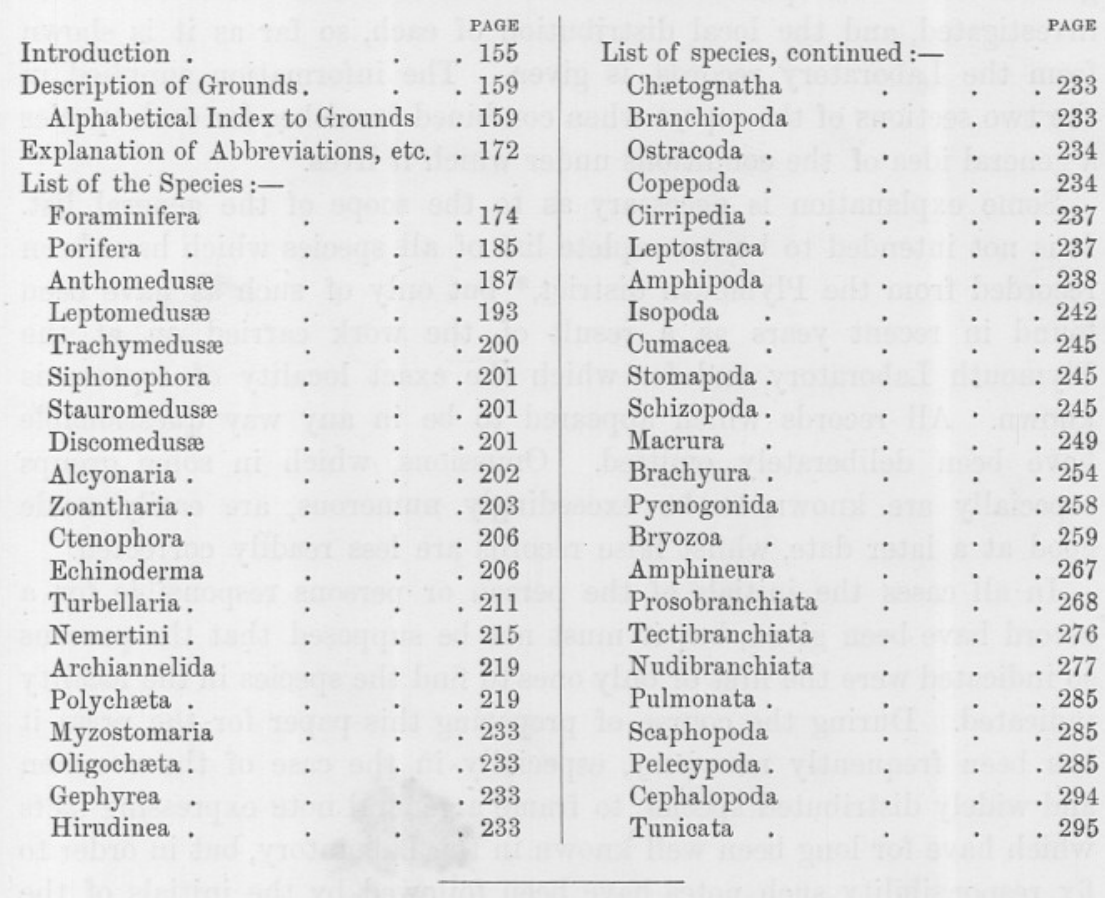

\section{INTRODUCTION.}

AFTER the publication in this Journal in 1899 of the paper "On the Fauna and Bottom-Deposits near the Thirty-Fathom Line from the Eddystone Grounds to Start Point" (Journ. Mar. Biol. Assoc., vol. v. pp. 365-542), observations were undertaken for the purpose of pre-

* It is hoped that main references to this paper may be made under " Marine Biological Association," and not under an author's name.-ED.

NEW SERIES.-VOL. VII. NO. 2. DEC. 1904. 
paring a similar account of the fauna of the inshore grounds in the neighbourhood of Plymouth. As the work proceeded, however, it was realised that, owing to the great damage which has been caused to the grounds within easy reach of the harbour by the constant discharge of mud and refuse from barges working in connection with the new dockyard extension at Devonport and other harbour works, any attempt to prepare a consecutive account of the fauna and bottom-deposits over the whole area, such as was given for the grounds from Eddystone to Start, would be a matter of extreme difficulty and of little use.

The present report has therefore been arranged in a somewhat different way. A general account is first given of certain typical areas, their physical conditions are briefly described, and a short list is added of the more common and characteristic species which are found in them. This description of the grounds is followed by a general list of the species which have been recorded in the whole area investigated, and the local distribution of each, so far as it is shown from the Laboratory records, is given. The information supplied in the two sections of the report when combined furnishes for each species a general idea of the conditions under which it lives.

Some explanation is necessary as to the scope of the general list. It is not intended to be a complete list of all species which have been recorded from the Plymouth district,* but only of such as have been found in recent years as a result of the work carried on at the Plymouth Laboratory and for which the exact locality of capture is known. All records which appeared to be in any way questionable have been deliberately omitted. Omissions, which in some groups especially are known to be exceedingly numerous, are easily made good at a later date, whilst false records are less readily corrected.

In all cases the initials of the person or persons responsible for a record have been given, but it must not be supposed that the persons so indicated were the first or only ones to find the species in the locality indicated. During the course of preparing this paper for the press it has been frequently necessary, especially in the case of the common and widely distributed species, to frame a general note expressing facts which have for long been well known in the Laboratory, but in order to fix responsibility such notes have been followed by the initials of the person by whom they were framed. In editing the notes, care has been taken that the collection of records for each species shall, as far as possible, give a not incorrect indication of the abundance and distribution of the species in the Plymouth district.

* Records for Plymouth previous to 1888 are summarised by Heape, "Preliminary Report upon the Fauna and Flora of Plymouth Sound," Journ. Mar. Biol. Assoc., Old Series, ii. 
Many of the records have already been printed in the Journal of the Association and elsewhere. It has not, however, been thought necessary in such cases to give detailed references, as a classified list of papers dealing with work done in the Laboratory has already been published (Journ. Mar. Biol. Assoc., vol. vi. p. 115).

As regards the question of nomenclature, whilst the names used are in general those which it is thought will be finally adopted by zoologists, we have not attached undue importance to this aspect of the matter, but by supplying in each case one reference to a good description (preference being given to one accompanied by a figure or to one in a recognised monograph of the group), we have endeavoured to leave no room for doubt as to the precise species which the name is intended to indicate.

The vast amount of detailed work which the preparation of this paper has entailed has been carried out chiefly by Mr. R. A. Todd and by Mr. S. Pace. A large amount of preliminary work in bringing the records together was carried out by $\mathrm{Mr}$. Todd. The whole, excepting Foraminifera and Worms, has been critically reviewed, extended, and prepared for the press by Mr. Pace, who has also paid special attention to the nomenclature and references employed.

Each group when completed has been submitted for critical examination to one or more specialists, to whom, as well as to the authors of the records, the best thanks of the Association are due for the help given.

Taking the groups in the order in which they are printed, the following notes as to the relative completeness of the lists, and as to those who have chiefly assisted in their preparation, may be of service.

Foraminifera. Our records for this group are almost all due to Mr. R. H. Worth, who has also arranged the list for publication. It has been submitted to Mr. J. J. Lister, who has made some valuable additions.

Porifera. The list is a very imperfect one, many common species not having been identified and recorded. The records that we have are chiefly due to Prof. E. A. Minchin and Mr. G. P. Bidder, both of whom have assisted in the revision.

HydrozoA. Most of the species which occur at all frequently are believed to have been included. The lists have all been revised by Mr. E. T. Browne, to whom we are indebted for many additions and suggestions.

Alcyonaria. Probably gives all the local species.

ZOANTHARIA. An incomplete list, no specialist on the group having worked at Plymouth.

Ctenophora. The only frequent species are included, but the number of records is not large. 
Echinoderma. Mr. Pace has devoted particular attention to this group, and the list given is an almost complete one, except for the Holothuria.

TurbelLaria. The records are almost all derived from Dr. F. W. Gamble's list published in this Journal (vol. iii. p. 30), and has been submitted to Dr. Gamble and Dr. E. G. Gardiner.

Nemertini. The list is based on that published in this Journal (vol. iii. p. 1) by Mr. T. H. Riches. It has been revised and added to by Mr. Riches, Mr. R. C. Punnett, and Mr. W. I. Beaumont.

Polycheta, ARchiannelida. The majority of the records are by Dr. E. J. Allen, by whom the list has been prepared. It is believed to be fairly complete for species which occur at all frequently, excepting in the families Syllidae and Maldanidae.

The list of Polynoidae is based on that published by Mr. T. V. Hodgson in this Journal (vol. vi. p. 218).

Myzostomaria, Gephyrea, Hirudinea, Oligocheta, Chetognatha. The lists of these groups are known to be incomplete.

Crustacea. The list of Branchiopoda is probably complete, that of the Ostracoda contains only a few records kindly supplied by the Rev. Dr. A. M. Norman, of which no account of the local distribution can be given, whilst that of the COPEPODA is confined to species for which Plymouth records have already been published by Mr. G. C. Bourne (Journ. Mar. Biol. Assoc., N.S., i. p. 144) and by Prof. P. T. Cleve (Svenska. Akad. Handl., vol. xxxiv., No. 2; Stockholm, 1900, etc.). The parasitic Copepoda recorded by. Dr. Bassett-Smith as occurring at Plymouth have been omitted, as they were probably not found upon fish caught within the area dealt with in this paper. The list of CirRIPEDIA is fairly complete.

The AMPHIPODA and IsOPODA are incomplete as regards the smaller forms, and do not contain records by anyone who has worked exhaustively at the groups in this district. CUMACEA are also incomplete. The Schizopoda, the account of which has been prepared by Mr. W. I. Beaumont, probably includes all the species which occur in any abundance.

The MACRURA and BrachyURA are fairly complete.

PyCNOGONIDA have not been revised by a specialist, and the list is imperfect.

BryozoA. The list has been submitted to Dr. S. F. Harmer, who has added many records.

Mollusca. The lists, which are chiefly based on the work of Mr. Todd and Mr. Pace, include all the more prominent forms, but are incomplete as regards the smaller and more critical species. The Tectibranchiata have been revised by Mr. W. I. Beaumont. 
Tunicata. The account is chiefly based upon the work of Mr. W. Garstang, but is by no means complete, as many of his records could not be made available in time for publication.

The description of the grounds has been prepared by Dr. E. J. Allen, with the assistance of Mr. S. Pace.

An alphabetical index of the grounds will be found below, and a list of all those zoologists by whom records have been made on p. 172 .

E. J. A.

\section{DESCRIPTION OF GROUNDS.}

\section{ALPHABETICAL INDEX.}

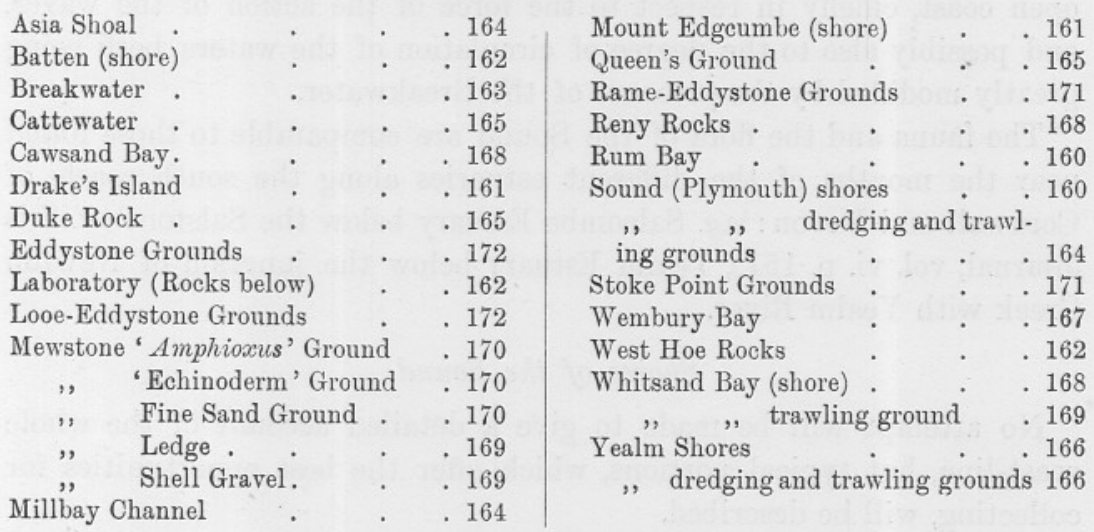

The area dealt with in this report extends from the shore to a depth of from 30 to 35 fathoms and may be considered as limited on the seaward side by a line running westwards from Bolt Head, passing a little outside the Eddystone Rocks and Hand Deeps, and then drawing northwards and meeting the coast at Looe. The length of such a line is about 35 miles, and the greatest breadth of the sea area included within it (from Plymouth to beyond the Eddystone) is from 14 to 15 miles. Roughly speaking, this area may be said to lie within a radius of 15 miles from the Laboratory. The area contains a number of typical tidal rivers (Hamoaze, Cattewater, Yealm R.), the large sheets of enclosed and sheltered sea represented by Plymouth Sound and the mouth of the Yealm, and a considerable stretch of coastal water exposed to the full force of the waves of the English Channel.

Before giving the detailed list of the fauna inhabiting the area, it will be convenient to review the general conditions prevailing in its different parts. In this way it is hoped that some conception, even if it be an imperfect one, of the nature of the influences which limit the distribution of the different species may be formed by those having no personal knowledge of the neighbourhood. 


\section{PLYMOUTH SOUND AND THE ADJACENT TIDAL RIVERS.}

Plymouth Sound must be regarded as an enclosed and sheltered arm of the sea, into which the two tidal rivers, the Tamar (with its estuary the Hamoaze) on the west, and the Plym (with its estuary the Cattewater) on the east, discharge their waters. The fauna and the flora of the Sound, in their general nature, are marine rather than estuarine. The typical estuarine species of the Hamoaze and Cattewater occupy no predominant place in their composition, and the effect of the fresh water entering the area does not appear to be great. On the other hand the conditions in the Sound differ from those obtaining on the open coast, chiefly in respect to the force of the action of the waves, and possibly also to the degree of circulation of the waters, both being greatly modified by the presence of the Breakwater.

The fauna and the flora of the Sound are comparable to those found near the mouths of the different estuaries along the south coasts of Cornwall and Devon: e.g. Salcombe Estuary below the Salstone ( $c f$. this Journal, vol. vi. p. 151), Yealm Estuary below the junction of Newton Creek with Yealm River.

\section{Shores of the Sound.}

No attempt will be made to give a detailed account of the whole coast-line, but typical portions, which offer the best opportunities for collecting, will be described.

The shores of the Sound are for the most part rocky, with gravel and stones between the rocks. Fine sand in small patches is exposed here and there at extreme low water, but there are no stretches of sandy shore.

Rum Bay. This term is used as a general name for the shore from Batten Breakwater to Jennycliff Bay.* It is a moderately sheltered stretch of coast, with low rocks of a very friable shale, which dip seawards and form ridges parallel to the line of coast. Near low-water mark the ground between the rocks is for the most part very stony, but fine sand and gravel are found at intervals. The low shale rocks are covered, between tide-marks, with Fucus, Pelvetia, Ascophyllum, and other brown weeds, occurring in definite zones, whilst at dead low water Laminaria is plentiful. The weeds and rocks are the home of the usual shore Gastropods of the district (Purpura lapillus, Patella vulgata, Littorina neritoides, L. rudis, L. littoralis, Gibbula cineraria, G. umbilicata, Calliostoma zizyphinus, Ocinebra evinacea), occurring each at its proper tidal level, as well as of the Polychætes Nereis pelagica

* The term Rum Bay, as here used, includes both Batten Bay and Rum Bay of the Admiralty charts. 
and Eulalia viridis. The overhanging ledges of rock give shelter to colonies of encrusting sponges (Hymeniacidon sanguineum, Halichondria panicea, and Sycon compressum), compound Ascidians (Botryllus violaceus, Botrylloides rubrum), and Polyzoa (Umbonula verrucosa, Crisia), upon, which Nudibranchs (Archidoris tuberculata, Aiolidia papillosa) feed.

On the Fucus the Hydroid Sertularia pumila, the Polyzoa Flustrella hispida and Membranipora pilosa, and Spirorbis borealis occur in quantity, and, on the Laminaria, Membranipora membranacea in large patches. Clava squamata and Coryne vaginata are also often plentiful.

The cracks and crevices between the layers of shale shelter a characteristic and typical fauna, of which Terebella lapidaria, Amphitrite gracilis, Polydora flava, Potamilla reniformis, and Petalostoma minutum are representative species of the higher and intermediate tidal zones, whilst Marphysa sanguinea and Polymnia nebulosa occupy a similar situation near low-water mark.

The patches of gravel and coarse sand contain at the higher tidal levels Audouinia tentaculata in large numbers, and the intermediate and lower zones are characterised by the presence of large Nephthys caeca, Sthenelais boa, Nereis cultrifera, and Glycera convoluta.

Patches of fine sand, the most productive of which lies immediately south of Batten Castle, are found to contain Arenicola marina, Lanice conchilega (in sheltered situations near rocks), Nephthys caca and $N$. Hombergi, Pectinaria Koreni, Scoloplos armiger, Magelona Fapillicornis, Pcecilochatus serpens, Scolelepis vulgaris (in black muddy sand), and a number of other sand-burrowing Polychætes, the burrowing brittle star Ophiocnida brachiata, and the Lamellibranchs Cultellus pellucidus and Tapes pullastra.

On stony ground the following species are met with in considerable abundance beneath the stones: Gammarus marinus, Porcellana platycheles, Cancer pagurus, Carcinus monas, Portunus puber, Nebalia bipes, Galathea squamifera, Lepidonotus clava and L. squamata, Amphivira elegans, Asterina gibbosa, Ophiothrix fragilis, Leptoplana tremellaris, Botryllus violaceus, Actinia equina, and during the winter months Goniodoris nodosa.

At the western end of Rum Bay, under Batten Castle, the shale joins the Plymouth limestone, and there is a mass of high rocks of the latter kind, with a fauna similar to that described in a subsequent paragraph.

Drake's Island and Mount Edgcumbe. From their position off the mouth of the Hamoaze Estuary, the shores of Drake's Island and Mount Edgcumbe are under the influence of tidal streams of considerable force, whilst at the same time they are sheltered from the southwest, and hence not exposed to the most violent wave action. The 
shores of Drake's Island are for the most part rocky, the rocks, composed of a hard grit, being generally steep and high. Between the rocks are small patches of stony ground, and of sand and gravels of various textures. The shores of Mount Edgeumbe resemble the stony patches on Drake's Island, and may be treated with them.

The fauna on the rock faces resembles that at Rum Bay, and needs no further detailed description beyond a notice of the fact that owing to the steeper character of the shore and the height of the rocks, the extent of surface exposed at each of the tidal levels becomes very much reduced, so that those animals which are restricted to a particular zone are represented by a relatively smaller number of individuals.

The overhanging ledges of rock are larger and more profusely covered than those at Rum Bay, but the same species are plentiful. Perhaps the most important addition to be made to the list given for Rum Bay is the Ascidian Clavelina lepadiformis, which during some summers has been very abundant beneath these ledges.

The characteristic fauna in the crevices of the soft shale at Rum Bay is not so marked at Drake's Island, though Marphysa sanguinea and Polymnia nebulosa are plentiful in crevices at dead low water.

On stony ground between the rocks at Drake's Island and along the Mount Edgcumbe shore, in addition to the species found under similar circumstances at Rum Bay, there may be found beneath the stones, more plentifully than at the latter locality, specimens of Cucumaria saxicola, Cucumaria Normani, and Echinus miliaris, whilst Myriothela phrygia is very frequent attached to the under surface of the stones.

On the south side of Drake's Island a patch of clean shell gravel is exposed, which is probably continuous with the shell gravel of Queen's Ground (cf. p. 165). In addition to numerous specimens of Carcinus monas, which are often of variegated colours matching the colour of the shell gravel, the crab Pirimela is found, whilst in the gravel Glycera gigantea occurs.

On the north-east side is a patch of sand, which is the most characteristic bit of clean, even-grained sand in the district. Its fauna includes Ammodytes, Natica Alderi, and Spisula (Mactra) solida.

A Zostera-bed exposed on the north side of the Island contains numerous Solen, occasional specimens of Echinocardium, whilst a patch of a few square yards of harder sand is crowded with Lutraria elliptica.

Limestone Shores (Rocks below Laboratory and under West Hoe; Rocks at Batten). The rocks on the limestone shores differ from the shale rocks which have been described at Rum Bay, in being much higher, in forming a large number of rock pools at the higher tidal levels, and in being of a much harder and closer texture, with few cracks and crevices. The rock fauna at the higher tidal levels, there- 
fore, shows special features on the limestone shores, whilst the fauna characteristic of the cracks and crevices between the layers of the shale is not represented. The upper portions of the rocks are densely covered with barnacles, amongst which the molluses Otina otis and Leuconia bidentata are plentiful. The sides of the rock pools are covered by growths of Coralline, red and green seaweeds, Hydroids such as Syncoryne gravata, Clava squamata, small Tubularia, and the sponges Leucosolenia botryoides, L. complicata, L. variabilis, and Clathrina coriacea. In certain of these pools the Archiannelid Dinophilus tceniatus is found in great numbers. Saxicava rugosa and Cliona celata are abundant, boring in the limestone.

In other respects the fauna on the limestone rocks does not greatly differ from that on the shale, the free-living animals being almost exactly the same. Some of the overhanging ledges are densely covered with the Ascidian, Styelopsis grossularia and Potamilla reniformis is very abundant.

The Breakwater. The most interesting feature of the fauna of the Breakwater is the collection of animals which are found boring in the limestone of which it is built. To such an extent is the stone eaten into by various animals that considerable damage is done to the structure, and constant repairs are called for. In a stone which has been injured through this cause the outer surface, to the depth of about a quarter of an inch, is converted into a honey-combed, friable mass through the ravages of the boring sponge Cliona celata, whilst at frequent intervals larger holes, each of which may have a diameter of a quarter of an inch, and may pierce the stone to the depth of one inch, are formed by the boring mollusc, Saxicava mugosa. To these two animals most of the damage is due, but in addition there are found a few holes formed by the molluse Gastrochana dubia, and many by the Polychætes Dodecaceria concharum, Polydora ciliata, Polydora hoplura, and Potamilla reniformis (the Sabella saxicava of Quatrefages). Dodecaceria forms holes of oval or figure of eight section, which may penetrate for a depth of several inches into the heart of the stone; Polydora ciliata forms small U-shaped burrows, open at each end, whilst $P$. hoplura makes similar burrows of larger size.*

Other features of the fauna of the Breakwater are the abundance of the anemone Corynactis viridis, and of a small salmon-red anemone at present undescribed, of Caryophyllia Smithi and of Galathea strigosa. Large nests of Lima hians have also been found there.

* The facts here recorded were investigated at the request of the Government engineers responsible for the repair of the Breakwater, and a report was furnished on the subject.-E. J. A. 


\section{Dredging and Trawling Grounds of the Sound.}

The greater part of Plymouth Sound consists of comparatively shallow water (4-6 fms.) with a bottom-deposit of fine muddy sand. Winding through this is a channel of much deeper water, which represents the old river-bed of the Tamar. After leaving the Hamoaze (the estuary of the Tamar) this deep channel turns northwards until it strikes the northern shores of the Sound close to Millbay, where, bending sharply to the eastward, it attains a depth of 23 fathoms. The channel continues to run eastwards, keeping within a short distance of the shore but gradually diminishing in depth until it reaches the Mallard Shoal, where, after passing southward between this and the Winter Shoal, it becomes lost in the shallow water forming the central part of the Sound (4-6 fms.). It is in this channel, and in the two channels at the eastern and western ends of the Breakwater, that stony ground suitable for dredging occurs. The greater part of the rest of the Sound, having a bottom of fine sand and mud, is better worked with trawls. The principal grounds referred to in the records are the following:-

Millbay Channel (14-23 fms.). The deep channel off Millbay is one of the most productive dredging grounds in the Sound. The dredge brings up masses of stones of varying size (chiefly limestone), with a few shells, all free from any growth of red or brown seaweeds. The stones are generally covered with a good deal of brownish mud, and are much honeycombed by the boring sponge Cliona celata, and by Saxicava rugosa, Polydora ciliata, and Dodecaceria concharum. Sponges, compound Ascidians, Antennularia antennina, and small Hydroids and Polyzoa are numerous; a great variety of small Polychætes, more especially Phyllodocids and Syllids, are hidden amongst the cavities on the surface of the stones, and masses of tubes of Filograna implexa are frequent.

The deepest part of the channel, which forms a deep pit or hole, is remarkable for the abundance of Antedon bifida, the dredge often coming up half full of these Echinoderms. From the sides of the pit Tubularia indivisa is often obtained in quantity.

Asia Shoal. Another productive dredging ground lies along the northern edge of the Asia Shoal, which is really the southern margin of the deep channel. The water is here shallower $(5-7 \mathrm{fms}$.) than in the Millbay Channel, the stones are chiefly the shales and grits of Drake's Island, instead of the honeycombed limestones found at Millbay, and they are generally covered with more or less mud. Red seaweeds grow on them in small quantities, and large Hydroids, especially Antenmularia antennina and Tubularia indivisa, are often abundant. 
Alcyonidium gelatinosum is sometimes present in large quantities, as well as Bowerbankia. Sponges also are numerous, and large numbers of the smaller Polychætes (Phyllodoce maculata, especially, may occur in great quantity) take refuge amongst the fixed organisms. Calyptroea chinensis is frequently found.

Queen's Ground. This term is used in the records to indicate the area extending from the Queen's Ground Buoy to the New Ground's Buoy and the ground around the latter. It is really the inner margin of the channel at the western entrance of the Sound. The depth is $5-6$ fms. The soil is mainly a coarse shell gravel, amongst which are a number of large shells and rather small flat stones. The ground is very clean, there being little mud covering the stones and shells, and the water is clearer and purer than the estuarine waters from the Hamoaze which run through Millbay Channel. The stones and shells afford attachment to occasional pieces of red seaweed (the rare Stenogramma may be especially noted) and to many of the larger Calyptoblastic Hydroids and branching Polyzoa (especially Bowerbankia). Lamellibranchs (especially Spisula) live amongst the shell gravel, whilst Portunus depurator is abundant on it. The Polychæte fauna differs considerably from that found on the edge of the Asia Shoal and in Millbay Channel.

Duke Rock. Depth 4-5 fms. The grounds around the Duke Rock form the western border of the channel at the eastern entrance of the Sound. In recent years the Admiralty have carried out extensive dredging operations in this neighbourhood, and have to a large extent diminished its value as a dredging ground for scientific purposes. The stones and shells which are taken here carry a similar collection of animals to that found at Queen's Ground, but the shell gravel, with the animals which inhabit it on the latter ground, is replaced by finer muddy sand.

Trawling Grounds of the Sound. The fine sand and mud grounds of the centre of the Sound and of Jennycliff Bay are best worked with small trawls of either shrimp mesh or mosquito mesh. Shrimps, prawns, and small crustacea, small flat-fishes, pipe-fishes, Sepiola atlantica, and Philine aperta, are the characteristic species taken. The dog-whelk (Nassa reticulata) and shore crab (Carcinus manas) are abundant species on these grounds, but are best taken in traps.

The Cattewater. The soil in the Cattewater (estuary of the Plym) below Turnchapel is all soft mud, which can be worked with a shrimp trawl. The characteristic local forms are almost exclusively shrimps, prawns (especially Palcemonetes), Mysis flexuosa, and Carcinus monas. The Cattewater is chiefly useful, however, as a collecting ground, from the fact that the Plymouth trawlers often throw a good deal of their-refuse 
overboard there, and many of the species from outside amongst this refuse are capable of surviving for a time.

\section{THE YEALM ESTUARY.}

In the Yealm Estuary is a large body of enclosed and sheltered water, with a fauna which is essentially marine for a considerable distance above the mouth. The mouth is almost closed by a bar of sand, a deep channel being left only on the southern side. At a distance of about a mile from the mouth, the estuary divides into two branches, the Yealm River proper and Newton Creek. The Yealm possesses a number of rich collecting grounds, which would well repay a more careful and detailed study than they have yet received.

\section{Shores.}

Yealm Sand-bank. This name has been used in the records to indicate a bank of fine to medium sand on the left bank of the Yealm River above the junction with Newton Creek, which is uncovered at low spring tides. The fauna is characterised by the presence in the sand of large numbers of Ensis ensis. By digging may also be obtained in more or less considerable numbers Synapta inhcerens, Tapcs pullastra and T. decussatus, Spisula solida, Gari depressa, Sigalion boa, Amphitrite gracilis, and large specimens of Nephthys cceca. On the surface of the bank are found Calliostoma zizyphinus, and, during the summer months, Aplysia punctata, both in considerable quantities.

Eastern Shore below junction of Yealm River and Newton Creek. Along this shore the soil is composed of a coarse, muddy gravel, the most striking feature of the fauna of which is the abundance of the large Terebellid Amphitrite Johnstoni, with its commensal Polynoid, Gattyana cirrosa. Scalibregma inflatum is also found here.

Zostera Bed near the Mouth of the Yealm. Along the southern shore a Zostera bed is just exposed close to the mouth of the estuary. The muddy sand in which the Zostera is rooted contains an abundant Polychæte fauna, of which the two most numerous species are Aonides oxycephala and Marphysa Belli, whilst Notomastus rubicundus and $N$. latericeus are also found.

\section{Dredging and Trawling Grounds.}

The channel of the river just below the junction of the Yealm and Newton Creek is the best dredging ground in the estuary. The bottom is covered with stones and shells (chiefly oyster-shells), to which red and brown seaweeds are attached in considerable quantities. Echinus 
miliaris is often abundant, and both Asterias rubens and A. glacialis are generally taken as well as Ophiothrix fragilis. In addition to the ordinary shallow-water crabs, Pilumnus hirtellus, Portunus arcuatus, Macropodia rostratus, Inachus dorynchus, and Hyas araneus are usually found. Large specimens of Archidoris tuberculata are met with, and Acmcea virginea, Calyptrcea chinensis, and Acanthochites fascicularis, each in considerable numbers, are characteristic. Of Hydroids, large colonies of Plumularia pinnata are the most abundant, whilst Hydractinia echinata is plentiful on shells inhabited by Eupagurus Bernhardus. Large specimens of Phallusia mamillata are frequent, as well as specimens of Ascidiella aspersa. Polycirrus aurantiacus is very plentiful, whilst numbers of Phyllodocids and Syllids, as well as other small Polychætes, hide amongst the stones and shells. Eurylepta cornuta, Lineus marinus, and Prosthecerceus vittatus are also generally to be found.

In the Yealm River itself, above the junction with Newton Creek, there is a large oyster bed.

Zostera Bed along the Southern Shore.-A Zostera bed lies close to the southern shore at the mouth of the harbour, which can be most usefully worked with a shrimp trawl. Its fauna resembles that of the Zostera bed in Cawsand Bay, to be mentioned later. In addition to pipe-fishes, wrasse, and other small fishes, Macromysis flexuosa is abundant, as well as Hippolyte varians, Calliostoma striatum, Lacuna divaricata, and Haliclystus auricula, whilst small Anemonia sulcata are abundant attached to the Zostera.

\section{OUTSIDE GROUNDS.}

Shores.

Wembury Bay. The shores of this bay form one of the best collecting grounds on the open coast in the neighbourhood of Plymouth. A reef of high rocks (Church Reef and Blackstone Rocks) runs seawards in a south-westerly direction from in front of Wembury Church, forming deep overhanging ledges on the landward side, and leaving at low water many tide-pools both large and small. To the westward of this reef the shore is formed of stretches of low, weed-covered rocks alternating with patches of gravel and sand of different textures. The whole shore is exposed to almost the full force of the Channel waves, and the greater part of the fauna is found beneath the overhanging ledges and in other sheltered situations amongst the rocks and seaweeds, The fauna of the sand and gravel is not very extensive.

The general character of the fauna of the rock ledges and of the rock pools is similar to that found in corresponding situations within the Sound (e.g Rum Bay, Drake's Island), but there is greater profusion 
both of individuals and of species. The same shore Gastropoda are found in their respective tidal zones, and the ledges of rocks and under sides of stones are covered with the same species of sponges, ascidians, polyzoa, anemones, and hydroids. Of echinoderms the representative species, as on the shores of the Sound, are Amphiura elegans, Ophiothrix fragilis Echinus miliaris, and Cucumaria saxicola, all of which are found under stones, the two latter only at extreme low water.

Reny Rocks. A reef of exposed, weed-covered, low rocks running from the Shagstone to the mainland. The fauna resembles that of the rocks in Wembury Bay.

Whitsand Bay. An exposed shore which consists chiefly of fine shifting sand of a not very productive character. At intervals small reefs of low rocks run out amongst the sand, which form the home of a few ordinary rock-haunting species, and are specially characterised in places by the great abundance of the reef-building polychæte, Sabellaria alveolata. The rocky foundation in close proximity to an abundant supply of sand evidently furnishes to these worms the special conditions necessary for the formation of their masses of sand-built tubes.

\section{Dredging and Trawling Grounds.}

Cawsand Bay. Depth 3-5 fms. A characteristic inshore shallow bay with a bottom of fine sand. Being sheltered from the south-west, it is protected from the most violent and frequent gales, which in this district come from that direction, and is only visited by heavy seas during gales from the east. In the shallowest parts of the bay is an extensive bed of Zostera, with a characteristic fauna, this being one of the chief features which distinguishes the trawling grounds of Cawsand Bay, from those of the more exposed Whitsand Bay, to be presently mentioned.

The smaller Crustacea (Hippolyte varians, Macromysis flexuosa, Cumacea, etc.) are abundant, and specimens of Maia squinado, the common edible crab (Cancer pagurus), and the shrimp (Crangon vulgaris) are generally obtained. Sepiola atlantica is always present and often numerous, and in the summer months Sepia officinalis is often abundant. On the Zostera, small specimens of Anemonia sulcata are frequent, and Foraminifera, especially Polystomella crispa and Discorbina rosacea, are generally abundant. Nassa reticulata oceurs in quantity, often covered with Hydroids. Spisula elliptica and Ensis ensis are common buried in the sand.

The most characteristic feature of the fauna of this bay is, however, the fishes, which are not dealt with in the present report. These consist of flat-fishes (Soles, Plaice, Dabs, etc.), Skates and Rays, and several species of pipe-fish. 
Whitsand Bay. Depth 4-8 fms. Another example of a shallow sandy bay, but being open to the south and west, it is subject to much more disturbance from the waves than Cawsand Bay. There is here also a great variety of the smaller Crustacea and Sepiola atlantica is abundant. Corystes cassivelaunus and Astropecten irregularis, characteristic sand-dwelling species, are often taken in this bay, though much less frequently than in deeper water.

Mewstone Ledge. Depth $10-15$ fims. A ridge of soft, red conglomerate rock runs seawards in a southerly direction from the Mewstone. Over this ridge at a depth of 10 to $12 \mathrm{fms}$. it is possible to work a dredge, the dredge often breaking away and bringing to the surface pieces of the rock of considerable size. The rock is free from the growth of any seaweeds, but is well covered with Hydroids, Polyzoa, and sponges. The following species are common and typical of the fauna associated with this red rock: Eunicella verrucosa with Gephyra Dohrni and Tritonia plebeia living upon it; Alcyonium digitatum with Ovula patula; Caryophyllia Smithi with its associated barnacle Pyrgoma anglicum; Antennularia antennina and A.ramosa with Scalpellum vulgare ; small colonies of Sertularella Gayi and S.polyzonias; Plumularia pinnata and P. Catharina; Lafoea dumosa with occasional specimens of Myzomenia banyulensis attached; Cucumaria brunnea on the rock itself and on the attached Hydroids; occasional specimens of Antedon rosacea, Ophiothrix fragilis, Henricia sanguinolenta, and of the large Holothurian, Holothuria nigra; Ophiopsila aranea concealed in holes and crevices of the rock; Phallusia mamillata, Ascidiella scabra, and Ciona intestinalis (small specimens); the Polyzoa Crisia cornuta and Bugula flabellata in abundance, Alcyonidium gelatinosum, Bicellaria ciliata, small colonies of Cellaria fistulosa and C. sinuosa, and occasional large masses of Lepralia foliacea, amongst which a number of small Crustacea, especially large numbers of Porcellana longicornis, are to be found. The large Phyllodoce Paretti is also often found here. The red rock itself is bored by numbers of Pholadidea loscombiana.

Mewstone Shell Gravel. On either side of the Mewstone ledge, and probably in patches between the rocks and the ledge, the bottom soil is composed of a coarse shell gravel. In working a dredge over the ledge a mixed fauna, comprising the animals from the rocks and from the gravel, is generally obtained. The gravel itself may also be worked with a small trawl, the Agassiz trawl having been generally used in our work.

Species characteristic of this shell gravel are Holothuria nigra (often in considerable numbers), Cardium norvegicum, Spatangus purpureus, Glycimeris glycimeris, Lumbriconereis impatiens, Glycera gigantea, Aglaophenia myriophyllum, and Eurynome aspera. 
Mewstone 'Amphioxus' Ground. Depth 10-12 fms. About $1 \frac{1}{2}$ miles to the southward of the Mewstone (off Yealm Head) is a patch of shell gravel of finer texture than that last described, which is one of the few localities in the Plymouth district where Amphioxus lanceolatus has been found in numbers. The fauna of this shell gravel is limited, but very characteristic. In addition to Amphioxus, the following are typical species: Anapagurus lavis and Eupagurus cuanensis, inhabiting chiefly the shells of Turritella communis and Aporrhais pes-pelecani, associated with which Epizoanthus incrustatus is very frequent and the Hydroid Merona cornucopice is often found, especially on the Aporrhais shells; Phascolion strombi, not uncommon living in empty Aporrhais shells; Ebalia tumefacta and E. tuberosa, Glycera lapidum, and Onuphis conchilega.

Mewstone 'Echinoderm' Ground. 2-4 miles south of Mewstone. Depth 23-24 fms. A few years ago this was one of the most profitable grounds in the neighbourhood on which to shoot a trawl when it was desired to obtain a good collection of invertebrates. Recently it has been almost entirely ruined owing to the amount of mud and refuse tipped upon it by barges from Plymouth and Devonport. The bottom soil consists of a coarse muddy gravel. The trawl, after a successful haul, will contain large numbers of Echinus esculentus, together with a few $E$. acutus; numbers of Solaster papposus, Buccinum undatum, Eupagurus Bernhardus in Buccinum shells, some carrying Adamsia polypus, others Hydractinia echinata, Eupagurus Prideauxi, with Adamsia palliata; Pecten opercularis often in quantity, P. tigerinus not uncommon, and an occasional specimen of $P$. maximus; Galathea dispersa, Inachus dorsettensis, Macropodia longirostris, Ascidiella scabra, a few Asterias glacialis and $A$. rubens, and varying quantities of Sertularella Gayi and Cellaria sinuosa and C. fistulosa, according to the exact position of the haul.

Inside the 'Echinoderm' ground, between that ground and Yealm Head, in rather shallower water, there is a stretch of coarse, muddy, gravel ground, which is covered almost exclusively with the brittlestar Ophiothrix fragilis. A dredge hauled on this ground comes up full of these Echinoderms, a few specimens of large Ophiocoma nigra being mixed with them.

Fine Sand south of Mewstone. Depths 27-30 fms. From 5 to 7 miles south of the Mewstone is a frequently worked trawling ground with a bottom of fine clean sand. The fauna here closely resembles that found on the Inner Eddystone Trawling Ground ( $c f$. this Journal, vol. v. p. 389). Characteristic and typical species are: Astropecten irregularis, Aphrodite aculeata, Corystes cassivelaunus, Ophiura ciliaris, Dentalium entalis, Alcyonium digitatum attached to 
shells, Pecten opercularis (abundant in patches), Sertularella Gayi and S. polyzonias, Aglaophenia myriophyllum, Cellaria sinuosa and C. fistulosa, Ascidiella scabra, and Macropodia longirostris.

Stoke Point Grounds. Western boundary, Blackstone Point; eastern boundary, Revelstoke Church Cove; seaward extension, about $1 \frac{1}{2}$ miles. The ground shelves very regularly outside the $10-\mathrm{fm}$. line; inside this line it is very uneven; maximum depth, $22 \mathrm{fms}$.

In their general features these grounds present much similarity to the 'Mewstone grounds,' but they offer rather greater diversity of type within a given area; and perhaps partly as the result of this and also of the fact that they are exposed to the full sweep of the Channel tide, the fauna is considerably richer than it is on the Mewstone grounds.

The friable red rock characteristic of the Mewstone Ledge is met with again; and forms numerous more or less detached reefs, off Stoke Point. It is abundantly perforated by Pholadidea, and in the disused crypt of this molluse, the remarkable Ophiurid Ophiopsila aranea is plentiful. As at the Mewstone, the surface of the rock is very clean, and it affords attachment to Eunicella, Antennularia, Aglaophenia, Alcyonium digitatum, Caryophyllia, Tethya, etc.

Between the reefs of red rock are patches of very rich shell sand and gravel.

The grounds include an eastward extension of the Mewstone 'Echinoderm' ground, together with patches where Ophiothrix and Ophiocoma are abundant. In deeper water this gives place to typical 'Choetopterus' ground.

The Rame-Eddystone Grounds. This name has been used to indicate the grounds lying for 3 to 4 miles on either side of the line from Rame to Eddystone and at depths of from 25 to $30 \mathrm{fms}$. Two typical classes of grounds can be recognised in this area: (1) coarse grounds with a bottom soil of muddy gravel, on which Choetopterus is one of the most striking forms, and the chief Hydroids are Halecium halecinum and H. Beani; and (2) fine grounds with a bottom soil of fine sand, characterised by the abundance of Cellaria sinuosa and C. fistulosa and by the Hydroid Sertularella Gayi. The grounds are very patchy, and the two typical faunas are much intermingled, so that it is only occasionally and after a short haul that a fair representation of either of the two types of fauna is obtained.

Both classes of ground can be profitably worked with both the dredge and trawl. On both, Echinus esculentus and Pecten opercularis may be met with in large numbers in particular spots, and Asterias rubens and A. glacialis are generally distributed over the area. 
The following are typical species occurring on the two classes of grounds:-

COARse Grounds. Chatopterus variopedatus, Hyalincecia tubicola, Halecium halecinum and H. Beani, Atelecyclus septemdentatus, Ophiura albida, Ophiactis Balli, Venus fasciata, Tapes virgineus, and Ebalia tuberosa and E. tumefacta.

Fine Grounds. Cellaria sinuosa and C. fistulosa, Ophiura ciliaris, Sertularella Gayi and S. polyzonias, Echinocardium cordatum, Corystes cassivelaunus.

From the above description it will be seen that the fauna of the Rame-Eddystone grounds resembles very closely that of the grounds in the neighbourhood of the Eddystone, already described in this Journal (vol. v. p. 365 et seq.).

The Looe-Eddystone Grounds. This name has been applied to an extension westwards of the Rame-Eddystone Grounds. Depths 25-30 fms. The fauna is of a similar general character to that of the latter grounds, but is particularly rich, as the result probably of the presence of much rough and rocky ground intermingled with trawling ground.

The Eddystone Grounds. For a detailed description of these grounds see this Journal (vol v. p. 365).

\section{LIST OF THE SPECIES.}

\section{Explanation of Abbreviations.}

The authorities for the various records are indicated by their initials, of which the following is a complete list; those of members, or late members, of the staff are marked with an asterisk :-

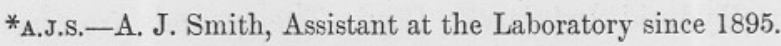

A.M.N.-The Rev. A. M. Norman, LL.D., F.R.s.

A.o.w.-A. O. Walker.

c.C.N.-Professor C. C. Nutting, University of Iowa.

c.S.-Professor Charles Stewart, LL.D., F.R.S., Conservator of the Royal College of Surgeons.

E.A.M.-Professor E. A. Minchin, M.A., Professor of Zoology, University College, London.

E.B.-Émile Brumpt, Lic. ès Sci., Paris.

E.G.G.-Dr. E. G. Gardiner, Woods Holl, Mass. 


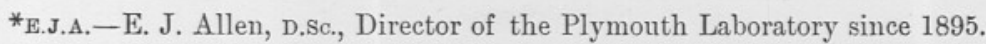

*E.J.B.-E. J. Bles, B.Sc., Director of the Plymouth Laboratory 1893-1894.

E.T.B.-E. T. Browne, B.A., University College, London.

${ }^{*}$ E.w.L.H.-E. W. L. Holt, Naturalist in Charge of North Sea Investigations 18921894; Hon. Naturalist at the Plymouth Laboratory 1897-1898; Scientific adviser to the Fisheries Branch of the Board of Agriculture for Ireland.

F.E.B.-F. E. Beddard, F.R.S., Prosector of the Zoological Society of London.

F.J.B.-Professor F. Jeffrey Bell, M.A., Assistant Keeper British Museum Natural History.

F.w.G.--F. W. Gamble, D.Sc., Lecturer in Zoology, Owen's College, Manchester.

*G.C.B. -G. C. Bourne, M.A., Director of the Plymouth Laboratory, 1888-1890.

G.P.B.-G. P. Bidder, M.A., Cambridge.

J.C.S.-J. C. Sumner, A.R.c.s.

J.J.L.-J. J. Lister, M.A., F.R.S., Fellow of St. John's College, Cambridge.

*J.T.c.-J. T. Cunningham, M.A., Naturalist in Charge of Fishery Investigations at the Plymouth Laboratory 1887-1894; ditto in charge of North Sea Investigations $1895-1896$.

${ }^{*}$ L н.G.--L. H. Gough, Рн.D., Assistant Naturalist in charge of Plankton Observations since November, 1902.

M.F.W.-The late Martin F. Woodward, A.R.C.S., Demonstrator in Zoology, Royal College of Science, London.

P.T.c.-Professor P. T. Cleve, University of Upsala.

R.c.P.--R. C. Punnett, M.A., Cambridge.

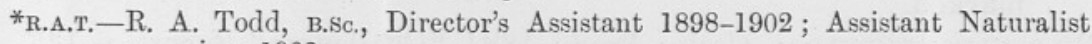
since 1902 .

R.G.-Robert Gurney, M.A.

R.H.w.-R. Hansford Worth, Plymouth.

R.M.P.-Mrs. S. Pace (Miss R. M. Clark).

S.F.H.--S. F. Harmer, D.Sc., F.R.S., Curator of the Museum of Zoology, Cambridge University.

*s.P.-S. Pace, Assistant Naturalist at the Plymouth Laboratory since Nov. 1902.

T.H.R.-T. H. Riches, M.A.

т.н.T.-T. H. Taylor, M.A., Lecturer in Zoology at the Yorkshire College, Leeds.

*T.V.H.-T. V. Hodgson, Director's Assistant at the Plymouth Laboratory 1895-1897.

w.B.B.-Professor W. B. Benham, D.sc., University of Otago, New Zealand.

w.E.H.--W. E. Hoyle, M.A., F.R.s., Curator of the Manchester Museum.

w.F.R.w.-Professor W. F. R. Weldon, F.R.s., Professor of Comparative Anatomy in the University of Oxford.

*w.G.-W. Garstang, M.A., Director's Assistant 1888-1890 ; Naturalist 1892-1894; ditto in charge of Fishery Investigations since 1897.

*w.H.-W. Heape, M.A., Superintendent of the Marine Biological Association, 1886-1888.

W.I.B.-.W. I. Beaumont, B.A.

w.P.M. -W. P. Marshall.

In some cases where there is only a single record, this has been indicated by a superior numeral, e.g. (J.T.c. ${ }^{1}$ ), or Queen's Gd. ${ }^{1}$

In the notes on Breeding Periods only those months are named for which definite records exist at the Laboratory. 


\section{FORAMINIFERA.}

BY

R. H. WORTH.

Miliolinæ.

(Biloculina Ringens (Lamarek): Williamson, 1858, Rec. For. Gt. Br., p. 79 , pl. vi, figs. 169,170 , pl. vii, fig. 171 .

Biloculina RINGEns, var. patagonica, Williamson, 1858, Rec. For. Gt. Br., p. 80 , pl. vii, figs. 175,176 . See also Biloculina elongata.

Cawsand B.; Drake's I.; Rame-Eddystone, generally distributed, but with unexplained preferences for certain localities, only occasionally plentiful; Eddystone-Looe, same remarks as last; Bolt, the species seems to be poorly represented.

Biloculina Depressa, d'Orbigny: Williamson, 1858, Rec. For. Gt. Br., p. 79 , pl. vii, figs. $172-174$ (as Biloculina ringens, var. carinata).

Rame-Eddystone, distribution similar to that of Biloculina ringens, but decidedly less numerous; Eddystone-Looe, same remarks as last; the species also occurs around the Bolt; [Drake's I. (J.J.L.)].

Biloculina elongata, d'Orbigny: Williamson, 1858, Rec. For. Gt. Br., p. 80 , pl. vii, figs. 175,176 (as Biloculina ringens, var. patagonica).

Five miles W. $\frac{1}{4}$ S. from Rame, characteristics exceptionally marked.

Biloculina tubulosa, Costa: Brady, 1884, "Challenger" Report, p. 147, pl. iii, fig. 6.

A few individuals have been somewhat doubtfully attributed to this species.

Spiroloculina Planulata (Lamarck): Williamson, 1858, Rec. For. Gt. Br., p. 82, pl. vii, fig. 178 (as Spiroloculina depressa, var. rotundata). Rame-Eddystone, generally distributed and fairly plentiful in places; Eddystone-Looe, same remarks as last; Bolt, an occasional spec.

Spiroloculina limbata, d'Orbigny: Williamson, 1858, Rec. For. Gt. Br., p. 82, pl. vii, fig. 177 (as Spiroloculina depressa).

Rame-Eddystone, follows distribution of last, but is less numerous; Eddystone-Looe, here the conditions seem somewhat changed, and this form is more numerous than planulata; [Drake's I. (J.J.L.)].

Spiroloculina tenuiseptata, Brady: Brady, 1884, "Challenger" Report, p. 153 , pl. $\mathrm{x}$, figs. 5,6 .

Rame-Eddystone, an occasional spec. only; Eddystone-Looe, an occasional spec. only.

Spiroloculina excavata, d'Orbigny: Brady, 1884, "Challenger" Report, p. 151 , pl. ix, figs. 5,6 .

Rame-Eddystone, generally distributed, but rare; EddystoneLooe, as above.

Spiroloculina fragilissima, Brady: Brady, 1884, "Challenger" Report, p. 149 , pl. ix, figs. $12-14$.

Found on the eastern slope of the Hand Deeps. 
[ Foraminifera-contd. ]

Miliolina trigonula (Lamarck): Williamson, 1858, Rec. For. Gt. Br., p. 84 , pl. vii, figs. $180-182$.

Drake's I.; Rame-Eddystone, generally distributed, but not numerous; Eddystone-Looe, scarce in the dredgings examined; Bolt, an occasional spec.

Miliolina tricarinata (d'Orbigny): Brady, 1884, "Challenger" Report, p. 165 , pl. iii, fig. 17 .

Rame-Eddystone, an uncommon form; Eddystone-Looe, none found as yet.

Miliolina oblonga (Montagu): Williamson, 1858, Rec. For. Gt. Br., p. 86, pl. vii, 186, 187, as M. seminulum, var. oblonga.

Rame-Eddystone, generally distributed, numerous everywhere, sometimes the dominant species in a dredging, one of the commonest Foraminifera; Eddystone-Looe, much as above, but the species is a little less frequent; Bolt, the species is less common in the dredgings examined; [Drake's I. (J.J.L.)].

Miliolina seminulum (Linné): Williamson, 1858, Rec. For. Gt. Br., p. 85 , pl. vii, figs. $183-185$.

Cawsand B.; Drake's I.; Rame-Eddystone, universally present, common everywhere, but numbers fluctuate considerably from point to point, not unusually one of the dominant species in a dredging; Eddystone-Looe, much as last, but never the dominant species so far as dredgings have been examined; Bolt, extremely common, the dominant species of the ground.

Miliolina subrotunda (Montagu): Brady, 1884, "Challenger" Report, p. 168 , pl. v, figs. 10,11 .

Drake's I.; Rame-Eddystone, common and almost universally distributed, but more plentiful in shallower water. These remarks apply to whole district.

Miliolina circularis (Bornem): Brady, 1884, "Challenger" Report, p. 169 , pl. v, figs. 13,14 .

Drake's I.; Rame-Eddystone, as last species, but less common; Bolt, occasional specimens.

Miliolina secans (d’Orbigny): Williamson, 1858, Rec. For. Gt. Br., p. 86 , pl. vii, figs. 188,189 (as M. seminulum, var. disciformis).

From the shallower dredgings throughout the district, seems to prefer clean sands, and is often common on beaches.

(Miliolina biconnis (Walker \& Jacob): Williamson, 1858, Rec. For. Gt. Br., p. 87, pl. vii, figs. 190-192.

Miliolina BICORnis, var. elegans: Williamson, 1858, Rec. For. Gt. Br., p. 88 , pl. vii, fig. 195 .

Cawsand B.; Drake's I.; Rame-Eddystone, generally distributed, always present, sometimes plentiful. The same remarks may safely be applied to the whole district.

Miliolina boueana (d'Orbigny): Brady, 1884, "Challenger" Report, p. 173 , pl, vii, fig. 173 .

As last species, but a trifle less common. 
[ Foraminifera-contd. ]

Miliolina agglutinans (d'Orbigny): Brady, 1884, "Challenger" Report, p. 180, pl. viii, figs. 6,7 .

Drake's I.; Rame-Eddystone, but commoner in shallow water; by no means an infrequent species anywhere in the district; occurs on Eddystone-Looe Gds., Bolt Gds., and all areas examined.

\section{Peneroplidinæ.}

Cornuspira foliacea (Philippi): Williamson, 1858, Rec. For. Gt. Br., p. 91, pl. vii, fig. 199 (as Spirillina).

Rame-Eddystone, generally distributed, some localities yield more specs. than others, but the form is very sparingly represented anywhere; Eddystone-Looe, rare.

Cornuspira involvens (Reuss): Brady, 1884, "Challenger" Report, p. 200 , pl. xi, figs. $1-3$.

Drake's I.; Rame-Eddystone, generally distributed, in some dredgings numbers were found adherent to Hydroids; EddystoneLooe, same remarks as last.

\section{Astrorhizidæ.}

HaLiphysema tumanowicziI, Bowerbank: Brady, 1884, "Challenger" Report, p. 281, pl, xxvii A, figs. 4, 5 .

Occurs but sparingly in the deeper dredgings everywhere in the district, is probably much commoner near the shore: [Queen's Gd., abundant at times (S.P.): Duke Rk. (E.J.A.)]

\section{Lituolinæ.}

Haplophragmium canariense (d'Orbigny): Williamson, 1858, Rec. For.

Gt. Br., p. 34, pl. iii, figs. 72, 73 (as Nonionina jeffreysii).

Drake's I.; Rame-Eddystone and all grounds, not uncommon anywhere, but shows a preference for more silty areas, at times quite plentiful.

Haplophragmium fontinense, Terquem: Brady, 188t, "Challenger" Report, p. 305, pl. xxxiv, figs. 1-4.

Occurs near the Eddystone, and possibly elsewhere on the outer grounds, but is nowhere common.

HAPlophragmiUm GLobigeriniforme (Parker \& Jones): Brady, 1884, "Challenger" Report, p. 312, pl. xxxv, figs. 10, 11.

Found in small numbers on the Rame-Eddystone Gd.

\section{Trochammininæ.}

Ammodiscus incertus (d'Orbigny): Williamson, 1858, Rec. For. Gt. Br., p. 93, pl. vii, fig. 203 (as Spirillina arenacea).

Rame-Eddystone, uncommon.

Ammodiscus GoRdialis (Jones \& Parker): Brady, 1884, "Challenger" Report, p. 333, pl. xxxviii, 7-9. Rame-Eddystone, rare.

Trochammina ochracea: Williamson, 1858, Rec. For. Gt. Br., p. 55, pl. iv, fig. 112, pl. v, fig. 113 (as Rotalina).

Found over the whole area, but is not plentiful anywhere. 
[ Foraminifera-contd. ]

Trochammina inflata (Montagu): Williamson, Rec. For. Gt. Br., p. 50, pl. iv, figs. 93, 94 (as Rotalina).

Distributed much like Haplophragmium canariense, which in some estuarine waters it largely replaces; it also occurs, however, well outside the 30 -fathom line.

\section{Textularinæ.}

Textularia sagittula, Defrance: Brady, 1884, "Challenger" Report, p. 361 , pl. xlii, figs. 17,18 .

The difficulty of precisely defining the limits of each species of the arenaceous textularia on these grounds is almost insuperable. Textularia sagittula is one of the less frequent forms, but it may occur anywhere where either gramen or agglutinans is found. The most typical specimens are found in estuarine waters.

Textularia trochus, d'Orbigny: Williamson, 1858, Rec. For. Gt. Br., p. 75 , pl. vi, figs. 160,161 (as T. cuneiformis, var. conica).

This form, like the last, is infrequent, but while some specimens of sagittula may be divided from agglutinans by fairly marked features, trochus, if it really occurs on these grounds, is always most suspiciously like a somewhat unorthodox gramen.

Textularia agglutinans, d'Orbigny: Brady, 1884, "Challenger" Report, p. 363, pl. xliii, figs. $1-3$.

Even between Textularia agglutinans and Textularia gramen there may arise difficulties of discrimination. Textularia agglutinans is every where the less common, but is generally distributed, and follows gramen in its preference of localities.

Textularia AgGlutinans, var. porrecta, Brady : Brady, 1884, "Challenger" Report, p. 364, pl. xliii, fig. 4.

This variety is occasionally found in all parts of the district.

Textularia Gramen, d'Orbigny: Brady, 1884, "Challenger" Report, p. 365 , pl. xliii, figs. 9,10 .

Cawsand B.; Drake's I. ; and the district generally.

Textularia gramen is much the most common of the Textularia; it frequently rises to from third to fifth in numerical order of the foraminifera taken in a dredging, and on the eastern slope of the Hand Deeps at one station it is only excelled by Miliolina seminulum.

Verneuilina polystropha (Reuss): Williamson, 1858, Rec. For. Gt. Br., p. 65 , pl. v, figs. 136, 137 [as Bulimina scabra (arenacea)].

Drake's I.; also sparingly present throughout the district, but this species shows a preference for shallow water. In estuaries it sometimes practically replaces the Textularia.

\section{Bulimininæ.}

Bulimina pupoides, d'Orbigny: Williamson, 1858, Rec. For. Gt. Br., p. 62 , pl. v, figs. 124,125 .

Drake's I. A common species throughout the district, a little more plentiful within than without the 25 -fathom line. 
[ Foraminifera-contd. ]

Bulimina marginata, d'Orbigny: Williamson, 1858, Rec. For. Gt. Br., p. 62 , pl. v, figs. $10-12$ (as B. pupoides, var. marginata).

Drake's I. Distribution precisely similar to that of Butimina pupoides, but individuals of the latter species average twice as numerous.

Bulimina aculeata, d'Orbigny: Williamson, 1858, Rec. For. Gt. Br., p. 62 , pl. v, fig. 128 (as B. pupoides, var. spinulosa).

Common throughout the district, follows closely the distribution of Bulimina pupoides, on the average one specimen of aculeata occurs to every twenty of pupoides. Williamson is probably right in making Bulimina marginata and Bulimina aculeata mere varieties of Bulimina pupoides, all intermediate forms occur.

Bulimina elegans, d'Orbigny: Brady, 1884, "Challenger" Report, p. 398 , pl. 1, figs. $1-4$.

Drake's I. Occurs sparingly wherever Bulimina pupoides is found, probably it should be regarded as a variety only.

Bulimina elegans, var. exilis, Brady: Brady, 1884, "Challenger" Report, p. 399, pl. l, figs. 5, 6.

5 miles W. $\frac{1}{4}$ S. from Rame; $4 \frac{1}{4}$ miles W. $\frac{1}{4}$ S. from Rame.

Bulimina elegantissima, d’Orbigny: Williamson, 1858, Rec. For. Gt. Br., p. 64 , pl. v, figs. 134,135 .

A minute form which is easily overlooked. On floating the silts, however, it is found in practically all dredgings; never numerically strong, it is none the less generally distributed.

Virgulina schreibersiana, Czjzek: Goës, 1894, Syn. Arctic and Scandinavian For., p. 48, pl. ix, fig. 459; Williamson, 1858, Rec. For. Gt. Br., p. 63, pl. v, fig. 131 (as Bulimina pupoides, var. compressa).

Generally distributed throughout the district. A small species liable to be underestimated as to numerical prevalence, unless the sands are floated; it is really present in considerable numbers almost everywhere.

Bolivina punctata, d'Orbigny: Brady, 1884, "Challenger" Report, p. 417 , pl. lii, figs. $18,19$.

Drake's I., and generally distributed throughout the district. See also note to Bolivina dilatata.

Bolivina textilaroides, Reuss: Williamson, 1858, Rec. For. Gt. Br., p. 77, pl. vi, fig. 168 (as Textularia variabilis, var. lovigata).

Drake's I., and generally distributed throughout the district, nowhere very numerous, nowhere rare.

I regard this and Williamson's Textularia variabilis, typica, as one species.

Bolivina DIFFormis (Williamson): Williamson, 1858, Rec. For. Gt. Br., p. 77, pl. vi, figs. 166, 167 (as Textularia variabilis, var. difformis).

A small form, easily overlooked, but well defined. It is generally distributed, but nowhere numerous. The prevalence of this species in surface townettings from the mouth of the Channel is a feature which must influence its apparent distribution on the sea-bottom. 
[ Foraminifera-contd. ]

Bolivina dilatata, Reuss: Williamson, 1858, Rec. For. Gt. Br., p. 76, pl. vi, figs. 164, 165 (as Textularia variabilis, var. spathulata).

Drake's I., and generally distributed over the district.

This species and Bolivina punctata both flourish on almost any bottom soil and are both happier on genuine muddy silts than most foraminifera. The relative prevalence of the two species obeys some so far undiscoverable law and is subject to wide variations.

Bolivina anariensis (Costa): Brady, 1884, "Challenger" Report, p. 423, pl. liii, figs. 10,11 .

Drake's I., and generally distributed throughout the district.

This is a species which will most probably be overlooked unless the silts are floated. Although common, it is not numerically well represented.

\section{Cassidulininæ.}

Cassidulina crassa, d'Orbigny: Williamson, 1858, Rec. For. Gt. Br., p. 69 , pl. vi, figs. 143,144 (as C. obtusa).

Drake's I. Universally distributed and fairly numerous everywhere.

Cassidulina Bradyi, Norman: Brady, 1884, "Challenger" Report, p. 431 , pl. liv, figs. $6-10$.

Distributed over whole district, but distinctly in less numbers than Cassidulina crassa.

\section{Lageninæ.}

Lagena globosa (Montagu): Williamson, 1858, Rec. For. Gt. Br., p. 8, pl. i, figs. 15, 16 (as Entosolenia).

A common form of universal occurrence.

Lagena apiculata, Reuss: Brady, 1884, "Challenger" Report, p. 453, pl. lvi, figs. $15-18$.

Somewhat rare.

Lagena Botelliformis, Brady: Brady, 1884, "Challenger" Report, p. 454 , pl. lvi, fig. 6 .

Not uncommon.

Lagena Levis (Montagu): Williamson, 1858, Rec. For. Gt. Br., p. 4, pl. i, figs. 5, $5 a$ (as L. vulgaris).

Present everywhere, but in less numbers than either Lagena orbignyana, globosa, marginata, or probably sulcata. Small specs. with either two or three chambers arranged in nodosarian form are not rare; some large individuals are entosolenian at one extremity and ectosolenian at the other.

Lagena clavata (d'Orbigny): Williamson, 1858, Rec. For. Gt. Br., p. 5, pl. i, fig. 6 (as L. vulgaris, var. clavata).

Generally distributed, but in considerably less number than Lagena lavis.

Lagena Gracillima (Seguenza): Brady, 1884, "Challenger" Report, p. 456 , pl. lvi, figs. $19-28$.

A not unusual form, has not been found in all dredgings, but probably is very generally distributed. 
[ Foraminifera-contd. ]

Lagena ASPERA, Reuss: Brady, 1884, "Challenger" Report, p. 457, pl. lvii, figs. 7-12.

Rare, but seems to exhibit a preference for the shallower waters.

Lagena Hispida, Reuss: Brady, 1884, "Challenger" Report, p. 459, pl. lix, figs. $2-5$.

Somewhat rare in the district.

Lagena lineata (Williamson): Williamson, 1858, Rec. For. Gt. Br., p. 9, pl. i, fig. 17 (as Entsolenia globosa, var. lineata).

Rame-Eddystone and Eddystone-Looe Gds., not a common form.

Lagena distoma, Parker \& Jones: Brady, 1884, "Challenger" Report, p. 461 , pl. lviii, figs. $11-15$.

Generally distributed, but in small numbers.

Lagena sulcata (Walker \& Jacobs): Brady, 1884, "Challenger" Report, p. 462 , pl. lvii, figs. $23,26,34$.

Drake's I., and throughout the district, a common species, occurring in moderate numbers.

LAGENA SULCATA, var. interrupta, Williamson.

[Drake's I. (J.J.L.).]

Lagena striata (d'Orbigny): Williamson, 1858, Rec. For. Gt. Br., p. 7, pl. i, fig. 14 (as L. vulgaris, var. substriata).

Drake's I., and throughout the district; a common species, and sometimes numerically strong.

Lagena semistriata, Williamson: Williamson, 1858, Rec. For. Gt. Br., p. 6, pl. i, fig. 9 (as L. vulgaris, var. semistriata).

Drake's I., and throughout the district; common.

LAGENA Gracilis, Williamson: Williamson, 1858, Rec. For. Gt. Br., p. 7, pl. i, figs. 12,13 (as $L$. vulgaris, var. gracilis).

Generally distributed.

Lagena squamosa (Montagu): Williamson, 1858, Rec. For. Gt. Br., p. 12 , pl. i, fig. 29 .

Drake's I., and throughout the district. Common and sometimes prominent in point of numbers.

Lagena Hexagona (Williamson): Williamson, 1858, Rec. For. Gt. Br., p. 13, pl. i, fig. 32 (as Entosolenia squamosa, var. hexagona).

Drake's I., and throughout the district. Common, perhaps not quite so plentiful as Lagena squamosa.

Lagena marginata, Walker \& Boys: Brady, 1884, "Challenger" Report, p. 476 , pl. lix, figs. $21,23$.

Generally distributed and in considerable numbers; small forms are sometimes difficult to discriminate from Lagena orbignyana.

LAGENa Marginata, var. ornata (Williamson): Williamson, 1858, Rec.

For. Gt. Br., p. 11, pl. i, fig. 24 (as Entosolenia).

Rare, but possibly many specs. have been overlooked.

Lagena trigono-marginata, Parker \& Jones: Brady, 1884, "Challenger"

Report, p. 482, pl. lxi, figs. 12, 13.

Rame-Eddystone, not infrequent, but represented by single specs. in the quantities of sand examined from the different dredgings. 
[ Foraminifera-contd. ]

Lagena quadrata (Williamson): Williamson, 1858, Rec. For. Gt. Br., p. 11, pl, i, figs. 27, 28 (as Entosolenia marginata, var. quadrata).

Present everywhere, but in small numbers. A mere variety of Lagena marginata.

Lagena orbignyana (Seguenza): Brady, 1884, "Challenger" Report, p. 484, pl. lix, figs. 24-26, etc.

Cawsand B.; Drake's I., and generally distributed over the whole district. This is distinctly the most common of the Lagenæ, and is well represented everywhere. The larger specimens are well characterised, but the smaller forms are sometimes extremely difficult to discriminate from Lagena marginata.

Lagena lagenoides (Williamson): Williamson, 1858, Rec. For. Gt. Br., p. 11, pl. i, figs. 25, 26 (as Entosolenia marginata, var. lagenoides).

Generally distributed and present everywhere, but not numerically strong in any dredging.

\section{Nodosariinæ.}

Nodosaria pyrula, d'Orbigny: Williamson, 1858, Rec. For. Gt. Br., p. 17 , pl. ii, fig. 39 .

Generally distributed, but somewhat scarce, and rarely represented by more than a few chambers of the shell

Nodosaria communis, d’Orbigny: Williamson, 1858, Rec. For. Gt. Br., p. 18, pl. ii, figs. 40, 41 (as Dentalina subarcuata).

Generally distributed, in moderate numbers. The larger specs. are the largest foraminifera of the district; one in especial, from one mile S.E. of Eddystone, measures $4 \mathrm{~mm}$. in length.

Nodosaria scalaris (Batsch): Williamson, 1858, Rec. For. Gt. Br., p. 15 , pl. ii, figs. $36-38$ (as $N$. radicula).

Drake's I., and generally distributed. Somewhat scarce at places.

Nodosaria calomorpha, Reuss: Brady, 1884, "Challenger" Report, p. 497 , pl. lxi, figs. $23-27$.

A small species, and very easily overlooked. Brady does not include it in his Synopsis of recent British Species. It is by no means uncommon in this district.

Cristellaria crepidula (Fichtel \& Moll): Williamson, 1858, Rec. For. Gt. Br., p. 29, pl. ii, figs. 56, 57 (as C. subarcuatula).

Drake's I., and generally distributed in the district; a common form, but not numerically strong.

Cristellaria rotulata (Lamarck): Williamson, 1858, Rec. For. Gt. Br., p. 27, pl. ii, figs. 52, 53 [as C. calcar (typica)].

Generally distributed, and somewhat more plentiful than $\mathrm{Cr}$ istellaria crepidula.

Cristellaria vortex (Fichtel \& Moll): Brady, 1884, "Challenger" Report, p. 548, pl. lxix, figs. 14-16.

Brady's only British locality is off the west coast of Scotland, and he states that the specs. are doubtfully referrible to this species. Occasional specs., the characteristics of which are well marked, are found in this district. 
[Foraminifera-contd. ]

Cristellaria italica (Defrance): Brady, 1884, "Challenger" Report, p. 544, pl. lxviii, figs. $17,18,20-23$.

Generally distributed, but scarce.

Amphiconyne FalX (Jones \& Parker): Brady, 1884, "Challenger" Report, p. 556, pl. lxv, figs. 7-9. Rare.

\section{Polymorphininæ.}

Polymorphina lactea (Walker \& Jacob): Brady, 1884, "Challenger" Report, p. 559, pl. lxxi, figs. 11, 14.

Drake's I., and generally distributed throughout district. Not a prominent species.

POLYMORPHINA GIBBA, d'Orbigny.

Included for present purposes in last-named species.

Polymorphina oblonga, Williamson: Williamson, 1858, Rec. For. Gt.

Br., p. 71, pl. vi, figs. $149,149 a$ (as P. lactea, var. oblonga). An occasional spec. from Rame-Eddystone Gds.

According to Spence Bate, this species was abundant near the

Eddystone in or before 1858. I should not call it other than somewhat scarce now; [on Zostera, Drake's I. (J.J.L.)].

Polymorphina COMPRessa, d'Orbigny: Brady, 1884, "Challenger" Report,

p. 565 , pl. lxxii, figs. $9-11$.

Occasionally found; [Drake's I. (J.J.L.)].

Uvigerina angulosa, Williamson: Williamson, 1858, Rec. For. Gt. Br., p. 67 , pl. v, fig. 140 .

Generally distributed and not uncommon, but never abundant.

\section{Globigerinidæ.}

Globigerina Bulloides, d'Orbigny: Williamson, 1858, Rec. For. Gt. Br., p. 56, pl. v, figs. 116-118.

Drake's I., and generally distributed throughout the district.

Universally present, and if never abundant at least never scarce.

Globigerina inflata, d'Orbigny: Brady, 1884, "Challenger" Report,

p. 601 , pl. lxxix, figs. $8-10$.

An occasional spec. only.

Orbulina universa, d'Orbigny: Williamson, 1858, Rec. For. Gt. Br., p. 2, pl. i, fig. 4 .

An occasional spec. only.

Spharoidina dehiscens, Parker \& Jones: Brady, 1884, "Challenger" Report, p. 621, pl. lxxxiv, figs. 8-11. Rare.

\section{Spirillininæ.}

SpIRILliNa vivipara, Ehrenberg: Williamson, 1858, Rec. For. Gt. Br., p. 92, pl. vii, fig. 202 (as S. perforata).

Drake's I. ; Rame-Eddystone, everywhere, but not plentiful.

Spirillina margaritifera, Williamson: Williamson, 1858, Rec. For.

Gt. Br., p. 93, pl, vii, fig. 204.

Rame-Eddystone, an occasional spec. 


\section{Rotalinæ.}

Patellina corrugata, Williamson: Williamson, 1858, Rec. For. Gt. Br., p. 46 , pl. iii, figs. $86-89$.

Generally distributed, but in small numbers.

Discorbina globularis (d'Orbigny): Williamson, 1858, Rec. For. Gt. Br., p. 53, pl. iv, figs. 104, 105 [as Rotalina concamerata (young)].

Cawsand B., at times abundant on the Zostera; [Drake's I. (J.J.L.)]. Generally distributed throughout the district, and in places common.

Discorbina rosacea (d'Orbigny): Williamson, 1858, Rec. For. Gt. Br., p. 54, pl. iv, figs. 109-111 (as Rotalina mamilla).

Cawsand B., very plentiful; Drake's I., and generally distributed. A common species, universally present, and frequently from fifth to sixth of all species in abundance. But both Discorbina globularis and Discorbina orbicularis appear to merge in this species, and few specimens are so well characterised as Williamson's figure.

Discorbina orbicularis (Terquem): Brady, 1884, "Challenger" Report, p. 647 , pl. lxxxviii, figs. $4-8$.

Common, and of universal distribution, but less abundant than either of the preceding Discorbinæ.

Discorbina PARISIEnsis (d'Orbigny): Brady, 1884, "Challenger" Report, p. 648 , pl. xc, figs. $5,6,9-12$.

Cawsand B., and sparingly represented in most dredgings, but at places apparently scarce.

Discorbina Bertheloti (d’Orbigny): Brady, 1884, "Challenger" Report, p. 650 , pl. lxxxix, figs. $10-12$.

Much as Discorbina parisiensis.

Planorbulina mediterranensis, d'Orbigny: Williamson, 1858, Rec. For. Gt. Br., p. 57, pl. v, figs. 119, 120 (as P. vulgaris).

Cawsand B.; Drake's I., and generally distributed through the district, but in very varying abundance. Sometimes third or fourth in numerical precedence, and sometimes very low indeed. There is no present key to its preferences of locality.

Truncatulina lobatula (Walker \& Jacob): Williamson, 1858, Rec. For. Gt. Br., p. 59, pl. v, figs. 121-123.

Cawsand B.; Drake's I., generally distributed throughout the district. Present practically everywhere, but in very varying abundance. Long ago Montagu noted it adherent to Hydroids, and this is the clue to its distribution; wherever Hydroids are present, this is one of the commonest foraminifera. It also exhibits an apparent preference for Sertularella gayi as a host.

Pulvinulina RePanda (Fichtel \& Moll): Williamson, 1858, Rec. For. Gt. Br., p. 52, pl, iv, figs. 101-103 [as Rotalina concamerata (mature)].

Cawsand B., and generally distributed, but not a species which occurs in abundance.

Pulvinulina Menardi (d’Orbigny): Brady, 1884, "Challenger" Report, p. 690 , pl. ciii, figs. $1,2$.

Somewhat uncommon, and doubtfully an inhabitant of the district, but rather a casual visitor. 
[ Foraminifera-contd. ]

Rotalia Beccaris (Linné): Williamson, 1858, Rec. For. Gt. Br., p. 48, pl. iv, figs. 90-92.

Cawsand B.; Drake's I.; everywhere throughout the district competes with Miliolina seminulum and, at places, Truncatulina lobatula for first place in abundance. On fine sands it seems best at home, and one dredging, about $4 \mathrm{~m}$. W. of Rame, in $20 \mathrm{fms}$., yielded close on 12,000 individuals of this species to each 10 grms. of bottom deposit, while Miliolina seminulum was present in equal numbers.

Rotalia nitida, Williamson: Williamson, 1858, Rec. For. Gt. Br., p. 54, pl. iv, figs. 106-108.

A common form of general distribution; never attaining the occasional extreme abundance of Rotalia beccarii, it none the less runs that species very nearly at places.

\section{Polystommellinæ.}

Nonionina depressula (Walker \& Jacob) : Brady, 1884, "Challenger" Report, p. 725, pl. cix, figs. 6, 7 .

Cawsand B.; Drake's I., and generally distributed. A common species, present everywhere, and frequently in some abundance.

Nonionina umbilicatula (Montagu): Williamson, 1858, Rec. For. Gt. Br., p. 32, pl. iii, figs. 68,69 (as $N$. barlecana).

Apparently prefers the deeper waters of the area examined; is nowhere a prominent species. Since, however, it also occurs in estuaries, it evidently is not restricted to deeper waters only.

Nonionina tURgida (Williamson): Williamson, 1858, Rec. For. Gt. Br., p. 50, pl. iv, figs. 95-97 (as Rotalina).

Cawsand B., and generally distributed. A small form probably present everywhere, but not abundant.

NONIONINA SCAPHA (Fichtel \& Moll): Brady, 1884, "Challenger" Report, p. 730, pl. cix, figs. 14-16.

Occurrence similar to that of Nonionina turgida.

Nonionina stelligera, d'Orbigny: Brady, 1884, "Challenger" Report, p. 728 , pl. cix, figs. $3-5$.

Cawsand B.; Drake's I., generally distributed, with an apparent preference for the shallower waters. Nowhere present in such numbers as Nonionina depressula.

Polystomella crispa (Linné): Williamson, 1858, Rec. For. Gt. Br., p. 40 , pl. iii, figs. $78-80$.

Cawsand B.; Drake's I., and generally distributed within the $15 \mathrm{fm}$. line; outside the $20 \mathrm{fm}$. line this form is practically unknown; never abundant outside 10 fms., but within that limit sometimes the dominant species. The Zosterc of Cawsand B. is at times crowded with this species.

Polystomella subnudosa (Münster): Brady, 1884, "Challenger" Report, p. 734, pl. cx, fig. 1.

Not infrequent outside $28 \mathrm{fms}$.

Polystomella macella (Fichtel \& Moll): Brady, 1884, "Challenger" Report, p. 737, pl. ex, figs. 8, 9, 11.

Drake's I., and probably wherever Polystomella crispa flourishes. 
[Foraminifera-contd: : Porifera ]

Polystomella striatopunctata (Fichtel \& Moll): Williamson, 1858, Rec. For. Gt. Br., p. 42, pl, iii, figs. 81,82 (as P. umbilicaluta).

Cawsand B.; Drake's I., and generally distributed, by no means uncommon at the $30-\mathrm{fm}$. line and outside.

Polystomella arctica, Parker \& Jones: Brady, 1884, "Challenger" Report, p. 735, pl. cx, figs. 2-5.

Drake's I.; Cawsand B., and throughout the district, but much less abundant than either Polystomella crispa or Polystomella striatopunctata; a complete series of intermediate forms connects these three species.

\section{PORIFERA.}

\section{Leucosoleniidæ.}

Leucosolenia botryoides (Ellis \& Solander): J. S. Bowerbank, Monogr. Brit. Spongiadæ, vol. iii, pp. 7, etc., fig.

On the shore between tide-marks, not abundant except in certain localities (E.A.M.): rocks under the Hoe, in abundance (G.P.B.): Wembury B., very abundant, on seaweeds, together with Sycon compressum (E.A.M.).

Leucosolenia complicata (Montagu).

Fairly common in rock-pools between tide-marks; also in deeper water off the Mewstone and elsewhere (E.A.M.).

Leucosolenia variabILIS, Haeckel.

Common everywhere in rock-pools between tide-marks (E.A.M.).

\section{Clathrinidæ.}

Clathrina coriacea (Fleming): J. S. Bowerbank, Monogr. Brit. Spongiadæ, vol. iii, pp. 8, etc., fig. (as Leucosolenia).

Found in small quantities nearly everywhere on rocks between tide-marks; near ladies' bathing-place; on rocks under the pier, etc. (E.A.M.): rocks under the Hoe, in abundance (G.P.B.): Reny Rks. (W.G. \& R.A.T.).

Clathrina lacunosa (Johnston): J. S. Bowerbank, Monogr. Brit. Spongiadæ, vol. iii, p. 9, fig. (as Lencosolenia).

Occasionally (w.G.): Rame-Eddystone Gds., not uncommon (s.P.): only below tide-marks and in deep water (E.A.M.).

\section{Syconidæ.}

Sycon COMPressum (Fleming): J. S. Bowerbank, Monogr. Brit. Spongiadæ, vol. iii, p. 1, fig. (as Grantia).

Common at low water on most rocky stations (s.P.): Millbay Docks, large specs. (E.J.A., R.A.T., S.P.).

This form appears to be an annual. The ova develope in Feb., free larvæ occur in July, and sponges $3 \mathrm{~mm}$. long in Sept. (G.P.B.).

Sycon cononatum, Ellis \& Solander: J. S. Bowerbank, Monogr. Brit. Spongiadæ, vol. iii, pp. 3, ete., fig. (as Grantia ciliata).

Common at most rocky stations at low water (s.P., E.A.M.): occurs in less exposed situations than $S$. compressum; common under the Hoe except in autumn (G.P.B.). 


\section{Halisarcidæ.}

Halisarca DuJardini, Johnston : F. E. Schulze, Zeitschr. wiss Zool,, vol. xxviii, p. 36, fig.

Millbay Ch. (G.P.B.).

\section{Haploscleridæ.}

Chalina oculata (Pallas): J. S. Bowerbank, Monogr. Brit. Spongiadæ, vol. iii, pp. 169 , etc., fig.

Millbay Pit; Wembury B. (т.V.H.).

Halichondria panicea (Pallas): J. S. Bowerbank, Monogr. Brit. Spongiadæ, vol. iii, pp. 97 , etc., fig.

Common; Rum B. ; Drake's I. (R.A.T.).

Apparently an annual (G.P.B.).

\section{Pœciloscleridæ.}

Leptolabis Luciensis, Topsent: E. Topsent, Result. Sci. Monaco, pt. $\mathrm{xxv}$, p. 185 , fig.

Plymouth (E.A.M.). Axinellidæ.

Hymeniacidon sanguineum (Grant): J. S. Bowerbank, Monogr. Brit. Spongiadæ, vol. iii, pp. 81, etc., fig.

Rocks under the Hoe, in abundance (G.P.B.).

Apparently an annual (G.P.B.).

Tragosia infundibuliformis (Johnston): J. S. Bonverbank, Monogr. Brit. Spongiadæ, vol. iii, pp. 137, etc., fig. (as Isodictya).

Looe-Eddystone Gds. (S.P.).

Tragosia polypoides, O. Schmidt: J. S. Bowerbank, Monogr. Brit. Spongiadæ, vol. iii, pp. 137, etc., fig. (as Isodictya dissimilis).

Mewstone Ledge (G.P.B.).

\section{Suberitidæ.}

Suberites domuncula, Olivi: J. S. Bowerbank, Monogr. Brit. Spongiadæ, vol. iii, pp. 91, etc., fig. (as Hymeniacedon suberea).

Millbay Ch. (т.V.H., R.A.T., G.P.B.) : Cattewater (т.v.H.) : Mewstone Gds. (т.v.H., R.A.T., G.P.в.): Eddystone Gds., Rame-Eddystone Gds. (E.J.A.): Yealm R. (E.J.A., T.V.H.).

\section{Polymastiidæ.}

Polymastia mamillaris (O. F. Müller): G. C. J. Vosmaer, Bijdr. Dierk., vol. xii, pt. v, p. 14.

Millbay Ch.; Mewstone Ledge ; Yealm R. (т.v.H.) : $4 \frac{1}{2}$ m. S.E. of the Mewstone (G.P.B.). Clionidæ.

Cliona celata, Grant: J. S. Bowerbank, Monogr. Brit. Spongiadæ, vol. iii, pp. 95 , etc., fig.

Millbay Ch.; Queen's Gd. (т.v.H.): Millbay Dock, common on piles (R.A.T.): Mewstone Gds. (T.V.H., R.A.T.): Eddystone Gds. (E.J.A.): Rame-Eddystone Gds. (E.J.A.).

\section{Tethyidæ.}

Tethya lyncurium (Linnæus): J. S. Bowerbank, Monogr. Brit. Spongiadæ, vol. iii, pp. 38, etc., fig.

Mewstone Ledge (T.V.H., S.P.). 


\section{ANTHOMEDUS尼.}

( $H Y D R O I D$ STAGE.)

\section{Clavidæ.}

Clava (sp.?) Leptostyla, Agassiz: W. Garstang, Journ. Mar. Biol. Assoc., ser. 2, vol. iii, p. 212.

Under the Hoe (w.G.).

Bearing gonophores in March (w.G.).

Clava multicornis (Forskål): G. J. Allman, Monogr. Gymnobl. Hydr., p. 246 , fig.

Common in tide-pools under the Hoe (G.c.B., w.G.): common

Wembury B., and inside Penlee (G.c.B.) : Millbrook (т.v.H.).

Bearing gonophores: Feb. (R.A.T.): Mar.-Apr. (w.G.): Nov. (T.V.H.).

Clava squamata (O. F. Müller): G. J. Allman, Monogr. Gymnobl. Hydr., p. 243, fig.

Fairly common between tide-marks; under the Hoe; Drake's I.;

Rum B.; etc. (E.J.A., R.A.T.): Jennycliff B. (A.J.S.): Barn Pool (E.T.B.):

St. Germans R., abundant on Fucus (w.G.).

Breeding: Feb., May (w.G.): Sept. (Е.т.в.).

Merona cornucopia, Norman: T. Hincks, Hist. Brit. Hydr. Zooph., p. 11, fig. (as Tubiclava).

Not uncommon on shells of Aporrhais, Dentalium, and Turritella, tenanted by Phascolion strombi (R.A.T., S.P.): Mewstone Gds. (W.G., R.A.T., S.P.): Stoke Pt. Gds. (R.A.T., S.P.): on the fine sand S. of the Eddystone (E.J.A.): Rame-Eddystone Gds. (R.A.T., S.P.).

Breeding: May (w.G., R.A.T.): June (W.G.).

Tubiclava Lucerna, Allman: G. J. Allman, Monogr. Gymnobl. Hydr., p. 256, fig.

Millbay Ch., on stones (E.J.A.).

\section{Hydractiniidæ.}

Hydractinia echinata, Fleming: G. J. Allman, Monogr. Gymnobl. Hydr., p. 345, fig.

Moderately common, on Buccinum and other shells inhabited by

Eupagurus Bernhardus (R.A.T., S.P.): occasionally taken on living Buccinum (R.A.T.): Cattewater (R.A.T., S.P.): Asia Sh., Jennycliff B. (R.A.T.): Cawsand B. (G.C.B., R.A.T., S.P.): Yealm R. (т.v.H., R.A.T.): 'Eddystone Gds. (E.J.A., S.P.): Mewstone Gds., Rame-Eddystone Gds, (R.A.T., S.P.).

Breeding: Aug. (w.G.): Nov. (s.P.).

\section{Podocorinidæ.}

Podocoryne carnea, M. Sars: G. J. Allman, Monogr. Gymnobl. Hydr., p. 349, fig.

Occasionally on old shells, 10-20 fms, (G.C.B.): Cawsand B., small colonies common on living $N$ assa reticulata (R.A.T.).

Medusæ liberated in May, 1896 (E.T.B.). 
[ Anthomedusæ-contd. ]

\section{Laridæ.}

Lar Sabellarum, Gosse [Medusa $=$ Willia stellata]: T. Hincks, Hist. Brit. Hydr. Zooph., p. 36, fig.

Millbay Ch., on Sabellid tubes (A.J.S.): on Potamilla Torelli (E.T.B.). Attached medusæ, Feb. (E.T.B., A.J.S.).

\section{Corynidæ.}

Coryne vaginata, Hincks: G. J. Allman, Monogr. Gymnobl. Hydr. p. 268 , fig.

Under the Hoe, fairly abundant (E.J.A.): common in tide-pools;

Rum B.; Cawsand B. (R.A.T.): Drake's I. (G.C.B., т.V.H.): inside

Bovisand Pier (w.G.): Bovisand B. (G.C.B.): Wembury B. (G.C.B., E.J.A.). Breeding: Apr. (R.A.T.): May-Aug. (G.C.B.).

Syncoryne eximia, Allman: G. J. Allman, Monogr. Gymnobl. Hydr. p. 282 , fig.

Single colony on piece of old rope, $1 \mathrm{~m}$. S. of the Mewstone,

May, 1889 (G.c.B.): Penlee Pt., Sept., 1897 (E.T.B.).

Medusa buds in September (E.T.B.).

Syncoryne (sp.?) gravata (T. S. Wright): T. Hincks, Hist. Brit. Hydr. Zooph., p. 53, fig.

Rocks under the Hoe, occasionally; Mt. Edgcumbe (W.G., R.A.T.):

Drake's I. ; Devil's Pt. ; Garden Battery (E.J.A.).

With attached medusæ: Mar. (W.G., R.A.T.): Apr. (E.J.A.).

Zanclea implexa (Alder): G. J. Allman, Monogr. Gymnobl. Hydr., p. 290, fig. (as Gemmaria).

A single colony on an encrusting Polyzoan dredged from rocky ground between Penlee Pt. and Rame Hd., Aug. 1899 (в.т.в.).

Medusæ liberated: Aug. (E.T.B.).

\section{Stauridiidæ.}

Stauridium productum, Wright: G. J. Allman, Monogr. Gymnobl. Hydr., p. 371, tig.

A small colony in a Laboratory tank, June, 1899 (E.T.B.).

\section{Myriothelidæ.}

Mrriothela phrygia, Fabricius: T. Hincks, Hist. Brit. Hydr. Zooph., p. 77 , fig.

Common, under stones, at low tide (G.C.B.): Drake's I. (G.C.B., R.A.T., S.P.): Millbay Ch. (E.J.A., A.J.S., T.V.H., R.A.T., S.P.): shore below the Lab., Mt. Edgeumbe (E.T.A.): Rame Hd. (т.V.H.): Asia Sh., Reny Rks. (R.A.T.): Picklecombe, Bovisand B., Mewstone (G.c.B.): Rum B. (A.J.S.): Wembury B. (G.C.B., T.V.H., A.J.S., R.A.T.).

Gonophores ripe: Jan., Mar., Apr. (R.A.t.): May-Aug. (G.c.в.).

\section{Eudendriidæ.}

Eudendrium album, Nutting: C. C. Nutting, Ann. Mag. Nat. Hist., ser. 7 , vol. i, p. 362, fig.

One of the most abundant Hydroids at Plymouth during the spring; on stones from Millbay Ch., often covering the stones 
[ Anthomedusæ-contd. ]

with a dense growth of white cottony tufts (C.C.N.): Millbay Ch. (E.J.A., T.V.H., A.J.S., R.A.T., S.P.): Asia Sh., Duke Rk. (R.A.T.).

Bearing gonophores: Jan., Feb. (A.J.s.): Mar. (т.v.H.): Apr. (c.c.N.): May (R.A.T.).

Eudendrium capillare, Alder: G. J. Allman, Monogr. Gymnobl. Hydr., p. 335 , fig.

Off Stoke Pt. on worm tubes, and on Antennularia antennina (G.C.B.): Eddystone Gds. (E.J.A.).

Eudendrium rameum (Pallas): T. Hincks, Hist. Brit. Hydr. Zooph., p. 80 , fig.

Eddystone, 30 fms., not common (G.C.B.).

Eudendrium ramosum (Linnæus): G. J. Allman, Monogr. Gymnobl. Hydr., p. 232, fig.

Not uncommon in dredgings from the Sound (R.A.T.): Reny Rks., at low tide (T.V.H.) : off the Mewstone, very common (G.c.B.) : RameEddystone Gds. (R.A.T.): Eddystone Gds., generally growing on shells or on other Hydroids, most frequent on sandy grounds (E.J.A.).

Breeding : Feb. (w.G.): Mar. (w.G., R.A.T., A.J.s.): Apr., May, Nov. (R.A.T.).

\section{Bougainvilliidæ.}

Bougainvillia ramosa (van Beneden): G. J. Allman, Monogr. Gymnobl. Hydr., p. 311, fig.

Drake's I., at low tide (G.C.B., R.A.T.): Millbay Pit; Millbay Dock, on piles; Asia Sh. (R.A.т.): Cawsand B., on old tin can (в.т.в.): Eddystone Gds., practically confined to the fine sand ground, where it is frequently met with, generally on Polychæte tubes or on other hydroids (E.J.A.).

Medusæ: Apr. (w.F.R.w.) : May (E.J.A.).

Perigonimus repens (T. S. Wright): T. Hincks, Hist. Brit. Hydr. Zooph., p. 90, fig.

Drake's I., on living Nassa reticulata (т.V.H.): Cawsand B., on Nassa ; Cattewater, large colony on abdomen of Carcinus (R.A.T.): Mewstone 'Echinoderm' Gd., inside Buccinum shell (E.T.B., R.A.T.) : Rame-Eddystone Gds. on Turritella shells (G.C.B.): Eddystone Gds., on claw of Eupagurus Bernhardus (E.J.A.).

Breeding: Aug. (w.G.) : medusæ in April (R.A.T.).

Perigonimus serpens, Allman: G. J. Allman, Monogr. Gymnobl. Hydr., p. 327 , fig.

$3 \mathrm{~m}$. S. of the Mewstone, on an old rope, Oct. 1897 ; Eddystone Buoy, Apr. 1898 (E.T.B.).

Medusa-buds present: Oct. (E.T.B.).

\section{Bimeriidæ.}

Garveia nutans, T. S. Wright: G. J. Allman, Monogr. Gymnobl. Hydr., p. 295 , fig.

Rocks under the Hoe (E.J.A., T.v.H., R.A.T.): on the piles of the Promenade Pier, occasionally (R.A.T.): Millbay Ch. and Pit (W.G., E.J.A., T.V.H., R.A.T.): Asia Sh. (R.A.T.).

Bearing gonophores: Feb.-Apr. (R.A.T.). 
[Anthomedusæ-contd. ]

Heterocordyle Conybearei, Allman: G. J. Allman, Monogr. Gymnobl. Hydr., p. 307, fig.

Abundant in the Sound on living Nassa reticulata (E.T.B., R.A.T.) :

Drake's I., on Nassa (T.V.H.) : Cawsand B., on Nassa (R.A.T., S.P.) :

Eddystone Gds., with Eupagurus (E.J.A.): near the East Rutts, on

Buccinum shells containing Eupagurus Bernhardus (E.T.B.).

With gonophores: Aug., Oct. (Е.т.в.).

\section{Tubulariidæ.}

Tubularia indivisa, Linnæus: G. J. Allman, Monogr. Gymnobl. Hydr., p. 400 , fig.

Rocks under the Hoe (E.J.A.): Drake's I., in low tide rock-pools (G.C.B.): Millbay Ch. and Pit, moderately common (W.G., E.J.A., R.A.T., S.P.): Asia Sh. (E.J.A., R.A.T.): Mt. Edgcumbe (E.J.A.): Duke Rk. (R.A.T.): Eddystone Rk. (E.J.A.).

Breeding: Feb.-Apr. (w.G., R.A.T.) : May (R.A.T.).

Tubularia (Thamnocnidia) humilis, Allman: $W$. Garstang, Journ.

Mar. Biol. Assoc., ser. 2, vol, iii, p. 212.

Tide-pools below the Hoe (w.G., T.V.H.): Barn Pool (w.G.).

Breeding in March (w.G.).

Tubularia (Thamnocnidia) larynx, Ellis \& Solander: G. J. Allman, Monogr. Gymnobl. Hydr., p. 406, fig.

Growing profusely on the Duke Rk. Buoy, and on other buoys in the East Ch., 1889 (G.c.B.): Millbay Ch. (w.G., R.A.T.): not taken in 1895 (E.J.A.).

Breeding: Apr.-May (w.G., R.A.T.) : Dec. (S.P.).

\section{Corymorphidæ.}

Corymorpha nutans, M. Sars [Medusa=Steenstrupia rubra, Forbes]: G. J. Allman, Monogr. Gymnobl. Hydr., p. 388, fig.

The hydroid generation has only been taken very occasionally, although its medusa is common (E.J.A.): off Ft. Tregantle, 5 specs. in about 3 fm., May 1887 (w.H.), 3 specs., May 1895 (E.J.A.): sought for unsuccessfully, 1889-90 (G.C.B.): two specs. on patch of sand in East Ch., May 1895 (E.J.A.): Queen's Gd., single spec., June 1904 (s.P.): Cawsand B., single spec., June 1904 (s.P.).

Attached medusæ in May.

\section{(MEDUSA STAGE.)}

\section{Codonidæ.}

Sarsia Eximia, Allman [Hydroid=Syncoryne]: G. J. Allman, Monogr. Gymnobl. Hydr., p. 282, fig.

Plymouth, some specimens in July (w.G.).

Sarsia Gemmifera: E. Forbes, Monogr. Brit. Naked-eyed Med., p. 57, fig. Plymouth; Sept., 1897; May, 1898; 1899, fairly common in June, few in July; Cawsand B., a young stage with medusa-buds, Sept. 1897 (E.T.B.). 
[ Bnthomedusæ-contd. ]

Sarsia Prolifera: E. Forbes, Monogr. Brit. Naked-eyed Med., p. 59, fig. Plymouth (w.G.): single spec. Aug., 1895; Whitsand B., about 100 specs., May 1896; May 1898, few ; July 1899, very abundant off Rame Hd. (E.T.B.).

Sarsia tubulosa (Sars): E. Forbes, Monogr. 'Brit. Naked-eyed Med., p. 55, fig.

Plymouth, rare (w.G.): very abundant about Saltash Bridge, Apr. 1898; occasionally taken in the Sound during May (E.T.B.).

Medusæ appeared in a Laboratory tank April 1898, and were reared to the adult stage (Е.T.в.).

Ectopleura Dumortieri, L. Agassiz: T. Hincks, Hist. Brit. Hydr. Zooph., p. 124, fig.

The Sound, a single spec. of an early stage (Е.т.в.).

Dipurena halterata: E. Forbes, Monogr. Brit. Naked-eyed Med., p. 53, fig. (as Slabberia).

Plymouth, Sept. 1893 ; July 1899, two early stages (E.T.B.).

Dipurena ophiogaster, Haeckel: G. J. Allman, Monogr. Gymnobl. Hydr., p. 45, fig. (as Sarsia strangulata).

Single spec., July 1897 (E.T.B.).

Perigonimus repens (T. S. Wright): T. Hincks, Hist. Brit. Hydr. Zooph., p. 90 , fig.

Plymouth (E.T.B.).

Medusæ budded off in tank in March (E.T.B.).

Steenstrupia Rubra, Forbes [Hydroid=Corymorpha nutans, M. Sars]: E. T. Browne, Proc. Zool. Soc., vol. 1896, p. 463, fig.

Abundant in spring and early summer (E.J.A.).

Hybocodon Prolifer, Agassiz: E. T. Browne, Proc. Zool. Soc., vol. 1896, p. 466.

Not uncommon Apr. 1898 (E.T.B.): few specs. with numerous buds, Apr. 1894 (w.G.).

Euphysa aurata : E. Forbes, Monogr. Brit Naked-eyed Med., p. 71, fig. Single spec. 4 m. S. of Breakwater, Sept. 1897; Apr.-June 1898, few specs. (Е т.в.).

\section{Tiaridæ.}

Amphinema dinema (Péron \& Lesueur): E. Haeckel, Syst. Medus., Denkschr. med.-naturw. Ges. Jena, vol. i, p. 50, fig. (as A. Titania).

Plymouth (w.G., E.T.B.) : Sept. 1893, fairly common; Sept. 1895, scarce; Sept. 1897, several specs. from the Sound and outside grounds; May 1898, two specs. near the Eddystone; June 1898, single spec. near the Mewstone; July 1899, common (Е.т.в.).

Tiara pileata, A. Agassiz: E. Haeckel, Syst. Medus., Denkschr. med.naturw. Ges. Jena, vol. i, p. 58, fig.

Plymouth (w.G.): Sept. 1897, 2 spees. 4 m. S. of the Breakwater and 2 specs. $5 \mathrm{~m}$. E. of the Eddystone, all young stages; MayJune 1898; June 1899, one or two specs. occasionally (Е.т.в.). 


\section{Margellidæ.}

[Anthomedusæ-contd. ]

Lizzia Blondina : E. Forbes, Monogr. Brit. Naked-eyed Med., p. 67, fig. Plymouth, Sept. 1897, of varying frequency in the Sound and ontside grounds, a large shoal off the Eddystone on Sept. 15th; May 1898, a large shoal in the Sound on May 2nd, it disappeared from the Sound on the 12th, and was off the Eddystone from the 16th to the 26th, but no specimens were then seen in the Sound or near the Mewstone; 1899, June-Aug., very scarce (E.T.B.).

Podocoryne carnea, M. Sars: G. J. Allman, Monogr. Gymmobl. Hydr., p. 349 , fig.

Free-swimming medusæ in March (w.F.R.w.); July-Aug. (w.G.).

Cyteandra areolata, Haeckel: E. T. Browne, Proc. Zool. Soc., vol. 1897, p. 817 , fig.

Sept. 1897, intermediate stages occasionally met with in the Sound and outside; June 1898, single spec. $10 \mathrm{~m}$. S. of the Mewstone (Е.T.B.).

Margelis autumnalis: C. Hartlaub, Wiss. Meeresunters., ser. 2, vol. ii, p. 465, fig. (as Bougainvillia).

Sept. 1897, very scarce; Sept. 1898, scarce (E.T.B.).

Margelis Bella: C. Hartlaub, Wiss. Meeresunters., ser. 2, vol. ii, p. 470, fig. (as Bougainvillia).

1898, a young stage in May, 4 specs. in June (E.T.B.).

Margelis britannica (Forbes): E. T. Browne, Proc. Liverp. Biol. Soc., vol. ix, p. 267.

Plymouth, end of April (E.T.B.).

Margelis PRinciPis, Steenstrup: E. Haeckel, Syst. Medus., Denkschr. med.-naturw. Ges. Jena, vol. i, p. 88, fig.

Plymouth, end of April (w.G.).

Margellium octopunctatum (M. Sars): E. Forbes, Monogr. Brit. Nakedeyed Med., p. 64, fig. (as Lizzia).

Numerous specs. during latter half of Feb. and March, 1893; less abundant in 1894 (w.G.): 1898, few specs. in Apr. and May (Е.T.в.).

\section{Cladonemidæ.}

Gemmaria implexa (Alder): T. Hincks, Hist. Brit. Hydr. Zooph., p. 59, fig. (as Zanclea).

Plymouth, 2 specs., Aug. 1895; Cawsand B., single spec. of an early stage, Sept. 1897 (E.T.B.).

Willia stellata [Hydroid=Lar Sabellarum, Gosse]: E. Forbes, Monogr. Brit. Naked-eyed Med., p. 19, fig.

Single spec., May 1896; Sept. 1897, earlier and intermediate stages frequently taken, adult very scarce; June-Aug. 1899, very scarce, only 2 specs. taken (Е.T.в.). 


\section{I.EPTOMEDUS㘴.}

(HYDROID STAGE.)

\section{Campanulariidæ.}

Clytia Johnstoni (Alder): T. Hincks, Hist. Brit. Hydr. Zooph., p. 143, fig.

Ubiquitous on Algæ and on other Hydroids (G.C.B.): L.W.35 fms. (R.A.T., S.P.).

Breeding: Mar. (R.A.T.): Apr. (A.J.S.): July (E.T.в.). Medusæ produced in Laboratory tanks, May 1895 (c.C.N.). Medusæ bearing gonothecæ, July (E.T.B.).

Agastra Caliculata (Hincks) $[$ Medusa $=A$. mir $\alpha$ (Hartlaub)]: $T$. Hincks, Hist. Brit. Hydr. Zooph., p. 164, fig. (as Campanularia).

The Hydroid form has not yet been recorded (s.P.).

Obelia dichotoma (Linnæus): T. Hincks, Hist. Brit. Hydr. Zooph., p. 156 , fig.

Rame Hd. (w.H.): Rame-Eddystone Gds., on Antennularia antennina and Sertularella Gayi (R.A.T.): Whitsand B., on wormtubes (G.C.B.): Eddystone Gds., on the fine sand area, growing on Pecten opercularis shells, Hydrallmania, Bougainvillia, Sertularia argentea, and Cellaria (E.J.A.).

Obelia geniculata (Linnæus): T. Hincks, Hist. Brit. Hydr. Zooph., p. 149 , fig.

Very common, generally on Laminaria (G.C.B., W.H., R.A.T., S.P.): Millbay Dock, on the piles (R.A.T.): Rame-Eddystone Gds., on Lepralia (R.A.T.).

Bearing gonophores: Mar.-Sept. (w.G.).

Obelia longissima (Pallas): T. Hincks, Hist. Brit. Hydr. Zooph., p. 154, fig.

From trawl refuse from outside the Eddystone (G.C.B.): S. of Eddystone (E.J.A.).

Obelaria gelatinosa (Pallas): T. Hincks, Hist. Brit. Hydr. Zooph., p. 151, fig. (as Obelia).

Lynher R., large colonies, up to 13 inches, in deep water under Sheviock Wood, July 1898 (E.w.L.H., W.I.B., E.T.B.): Bovisand B. (T.V.H.).

Campanularia angulata, Hincks: T. Hincks, Hist. Brit. Hydr. Zooph., p. 170 , fig.

Bovisand B., at low tide (G.C.B.).

Campanularia flexuosa (Hincks) : T. Hincks, Hist. Brit. Hydr. Zooph., p. 168, fig.

Very common on weeds and in rock-pools below the Hoe (G.C.B.): common on the shore between tide-marks; on hulks and buoys in the Sound and Cattewater; Phœnix Wharf and Millbay Dock, on piles: Millbay Pit (R.A.T.): Mt. Edgcumbe (E.J.A.): Saltash Pier, very abundant Oct. 1897 (E.T.B.).

Gonosomes well developed in May (c.C.N.). 
[ Leptomedusæ-contd. ]

Campanularia Hincksi, Alder: T'. Hincks, Hist. Brit. Hydr. Zooph., p. 162 , fig.

Common in 10-30 fms. on shells, Hydroids, Cellaria, etc.; RameEddystone Gds.; Mewstone Gds. (R.A.T.) : Eddystone Gds. (E.J.A.).

Campanularia neglecta (Alder): T. Hincks, Hist. Brit. Hydr. Zooph., p. 171, fig.

On stems of Tubularia indivisa; Millbay (C.C.N.).

Campanularia verticillata (Linnæus): T. Hincks, Hist. Brit. Hydr. Zooph., p. 167, fig.

Mewstone Gds.; Eddystone Gds., occasionally on the fine sand areas (E.J.A.).

Campanularia volubilis (Linnæus) : T. Hincks, Hist. Brit. Hydr. Zooph., p. 160 , fig.

Duke Rk., on shells (G.C.B.): N. of Breakwater, $5 \frac{1}{2}$ fms., plentiful (w.H.).

Gonothyraea Lovḱni, Allman: T. Hincks, Hist. Brit. Hydr. Zooph., p. 181, fig.

Millbay Ch.; Hamoaze (E.J.A.): Millbay Dock, on piles; on hulks in Cattewater; between tide-marks Turnchapel, Rum B., and Yealm R.; Asia Sh. (R.A.T.): Mt. Edgcumbe, at low tide (C.C.N., E.J.A.); Barn Pool on Fucus ; Saltash Pier (E.T.B.).

Breeding: Mar. (R.A.T.): Apr. (w.G., R.A.T.) : Sept. (в.T.в.) : Oct. (w.G., E.T.B.) : Nov. (W.G.)

Sometimes grows in great profusion in the Laboratory tanks (E.J.A.).

\section{Campanulinidæ.}

Campanulina Repens, Allman: T. Hincks, Hist. Brit. Hydr. Zooph., p. 189 , fig.

Winter Sh., ${ }^{1}$ abundant on Delesseria; between Penlee Pt. and Rame Hd., on Algæ and stems of Tubularia; Mewstone Gds., ${ }^{1}$ many colonies on a piece of rope (E.T.B.).

Gonophores: July (E.T.B.).

Opercularella hispida : C. C. Nutting, Ann. Mag. Nat. Hist., ser. 7, vol. i, p. 363 , fig.

The type specimen from Plymouth, on a stone associated with Clava multicornis (C.C.N.).

Opercularella lacerata (Johnston): 'T'. Hincles, Hist. Brit. Hydr. Zooph., p. 194, fig.

Millbay Dock, on young stems of Tubularia indivisa and on Eudendrium (C.C.N.).

\section{Lafoëidæ.}

LAFö̈a dumosa (Fleming) [cf. Coppinia arcta (Dalyell)]: T. Hincks, Hist. Brit. Hydr. Zooph., p. 200, fig.

Common in 15-35 fms., on shells, worm-tubes, other Hydroids, etc. (R.A.T., S.P.): Eddystone Gds., both the creeping and branched varieties, the latter especially upon the fine sand areas where it is attached to shells or Polychæte tubes (E.J.A.).

The Neomenian Myzomenia ( = Dondersia) banyulensis is frequently associated with the erect form of $L$, dumosa (E.J.A., S.P.). 
[ Leptomedusæ-contd. ]

Lafoëa fruticosa, M. Sars: T. Hincks, Hist. Brit. Hydr. Zooph., p. 202, fig.

Not uncommon, $15-30$ fms. (R.A.T., S.P.): Rame-Eddystone Gds. (E.T.B. \& R.A.T., S.P.) : Eddystone Gds. (E.J.A.): Stoke Pt. Gds. (G.C.B., S.P.).

Calycella syringa (Linnæus): T. Hincks, Hist. Brit. Hydr. Zooph., p. 206, fig.

Abundant on roots of Laminaria (G.C.B.): abundant on young stems of Tubularia indivisa (c.c.N.): Penlee Pt.; Rame Hd. (G.C.B.): Saltash Pier, on Sertularia (E.T.B.).

Calycella fastigiata (Alder): T. Hincks, Hist. Brit. Hydr. Zooph., p. 208, fig.

Eddystone Gds. (E.J.A.).

Cuspidella costata: T. Hincks, Hist. Brit. Hydr. Zooph., p. 210, fig.

Inner Eddystone trawling grounds (E.J.A.): growing on weed, trawl refuse (G.C.B.).

Cuspidella Grandis: T. Hincks, Hist. Brit. Hydr. Zooph., p. 210, fig.

On stems of Halecium tenellum (c.C.N.).

\section{Trichydridæ.}

Trichydra pudica, T. S. Wright: T. Hincles, Hist. Brit. Hydr. Zooph., p. 216, fig.

Eddystone Gds., fine gravel (E.T.B. \& E.J.A.): Eddystone Buoy (E.T.B.).

\section{Coppiniidæ.}

COPPINIA ARCTA (Dalyell) [?=gonosome of Lafoëa dumosa (Fleming)]: T. Hincks, Hist. Brit. Hydr. Zooph., p. 219, fig.

Eddystone Gds., abundant on the fine sand grounds; growing on Hydroids, especially Lafö̈a dumosa var. robusta and Sertularia abietina (E.J.A.): Mewstone Gds. (A.J.s.): Rame-Eddystone Gds.; Mewstone Ledge, very occasionally (R.A.T.).

\section{Haleciidæ.}

Halecium Beani (Johnston): T. Hincks, Hist. Brit. Hydr. Zooph., p. 224, fig.

Not uncommon in the Sound, on stones and shells, and outside, in 15-30 fms., on shells, other Hydroids, and on Chcetopterus tubes. Not so abundant as H. halecinum (R.A.T., S.P.): Eddystone Gds. (E.J.A.).

With gonophores: Jan. (w.G.) : Mar., Apr. (R.A.T.): May (s.P.): July (w.G.) : Oct. (R.A.T.).

Halecium halecinum (Linnæus): T. Hincks, Hist. Brit. Hydr. Zooph., p. 221, fig.

Not uncommon in the Sound, on stones and shells. Common outside, 15-30 fms., on Chotopterus tubes, stones, and shells (R.A.T., S.P.) : Eddystone Gds., abundant on certain grounds, and generally on Chatopterus tubes (E.J.A., S.P.): Rame-Eddystone Gds.; Mewstone Gds. (R.A.T., S.P.); Stoke Pt. Gds.; Looe-Eddystone Gds. (s.P.).

Breeding: Jan. (w.G.): Feb.-June (R.A.T.) : July (w.G.). 
[ Leptomedusæ-contd. ]

Halecium labrosum, Alder: T. Hincks, Hist. Brit. Hydr. Zooph., p. 225 , fig.

Eddystone Gds. (E.T.B.).

Halecium tenellum, Hincks: T. Hincks, Hist. Brit. Hydr. Zooph., p. 226 , fig.

Plymouth, 18 fms. (c.C.N.).

Breeding: Apr. (c.C.N.).

Haloikema Lankesteri: G. C. Bourne, Jour. Mar. Biol. Assoc., ser. 2, vol. i, p. 395, fig.

Duke Rk., on flat stones (G.C.B., E.T.B.): S. end of Jennycliff B. (G.C.B., W.G.).

\section{Sertulariidæ.}

Sertularella Gayi (Lamouroux) : Tr. Hincks, Hist. Brit. Hydr. Zooph., p. 237 , fig.

Not uncommon in the Sound (R.A.T., S.P.): Eddystone Gds., one of the most characteristic features of the fauna of the fine sand grounds (E.J.A., S.P.): Rame-Eddystone Gds.; Mewstone Gds. (R.A.T., S.P.): Stoke Pt. Gds.; Looe-Eddystone Gds. (s.P.).

Breeding: Feb. (w.G.): Mar. (R.A.T.) : Aug.-Oct. (w.G.).

Sertularella polyzonias (Linnæus): T. Hincks, Hist. Brit. Hydr. Zooph., p. 235, fig.

Occasionally in the Sound (R.A.T., S.P.): Eddystone Gds., with S. Gayi, but much less common (E.J.A., S.P.): Mewstone Gds.; RameEddystone Gds. (R.A.T., S.P.): Stoke Pt. Gds. (s.P.).

Breeding: Aug. (s.P.) : Sept. (R.A.T.)

Diphasia attenuata (Hincks): T. Hincks, Hist. Brit. Hydr. Zooph., p. 247 , fig.

On the fine sand of the 'Outer' Trawling Gds. (E.J.A.).

Diphasia pinaster (Ellis \& Solander): T. Hincks, Hist. Brit. Hydr. Zooph., p. 252, fig.

Very common in trawl refuse; S. of Eddystone (G.C.B.).

Diphasta Pinnata (Pallas): T. Hincks, Hist. Brit. Hydr. Zooph., p. 255 , fig.

5 m. S. of Eddystone (G.C.B.).

Breeding: Apr. (w.G.): gonophores in May (G.C.B.).

Diphasia rosacea (Linnæus): T. Hincks, Hist. Brit. Hydr. Zooph., p. 245 , fig.

Millbay Ch., common on stones (w.G.): Millbay Dock, on the piles (W.G., R.A.T.) : $2 \mathrm{~m}$. S. of the Mewstone, small colony on piece of rope (E.T.B.): Eddystone Gds., oceasionally met with (E.J.A.).

Breeding: Mar. (w.G., R.A.T.): Apr. (w.G.).

Diphasia tamarisca (Linnæus): T. Hincks, Hist. Brit. Hydr. Zooph., p. 254 , fig.

Eddystone Gds. (E.J.A.).

Sertularia abietina, Liunæus: T. Hincks, Hist. Brit. Hydr. Zooph., p. 266 , fig.

Common in trawl refuse (G.C.B.): abundant in depths below $30 \mathrm{fms}$. on the 'Outer' Trawling Gds. (E.J.A.): Millbay Ch., occasionally (R.A.T., S.P.). 
[ Leptomedusæ-contd. ]

Sertularia argentea, Ellis \& Solander: T. Hincles, Hist. Brit. Hydr. Zooph., p. 268, fig.

Queen's Gd.; Asia Sh.; Millbay Ch. and Pit (R.A.T., s.P.): Eddystone Gds., not infrequent on Pecten opercularis shells, and on other Hydroids (E.J.A.): Yealm R. (R.A.T.): Stoke Pt. Gds. (R.A.T., S.P.): Saltash Pier (E.T.B.).

Breeding: Feb. (w.G.): Mar., Apr. (R.A.T.).

Sertularia cupressina, Linnæus: T. Hincks, Hist. Brit. Hydr. Zooph., p. 270 , fig.

Common in trawl refuse from the Eddystone (G.C.B.): Queen's Gd. (R.A.T., S.P.).

Gonophores ripe: March (R.A.T.).

Sertularia operculata, Linnæus: T. Hincks, Hist. Brit. Hydr. Zooph., p. 263, fig.

Wembury B.; Eddystone Rk., abundant Apr. 1898; Eddystone Buoy (E.T.B.).

Sertularia pumila, Linnæus: T. Hincks, Hist. Brit. Hydr. Zooph., p. 260 , fig.

Abundant on rocks and weeds, especially Fucus, between tidemarks (G.C.B., R.A.T., S.P.): buoy near Breakwater (R.A.T.).

Breeding: Mar.-July (w.G.).

Hydrallmania falcata (Linnæus): T. Hincks, Hist. Brit. Hydr. Zooph., p. 273 , fig.

Not uncommon in the Sound; common, $15-30$ fms., on stony ground, and on sand with stones and shells; always attached to stones or shells (R.A.T.): abundant in the Hamoaze (E.J.A.): Millbay Pit; Duke Rk.; Mewstone Gds.; Cawsand B.; Rame-Eddystone Gds. (R.A.T.) : Eddystone Gds., occasional specs. (E.J.A.).

Breeding: Jan. (w.G.) : Feb.-Apr. (R.A.T.).

Thuiaria articulata (Pallas): T. Hincks, Hist. Brit. Hydr. Zooph., p. 277 , fig.

Wembury B.; stones and shells off Mewstone (G.c.B.): Mewstone Ledge (R.A.T.).

\section{Plumulariidæ.}

Antennularia antennina (Linnæus): T. Hincks, Hist. Brit. Hydr. Zooph., p. 280, fig.

Not uncommon in the Sound; common outside, 15-30 fms., especially on medium gravel and muddy sand (R.A.T., S.P.): Eddystone Gds. (E.J.A., S.P.): Cawsand B ; Rame-Eddystone Gds.; Mewstone Gds. (R.A.T., S.P.): Stoke Pt. Gds.; Looe-Eddystone Gds. (s.P.).

Breeding: Apr. (R.A.T.): May (w.G., R.A.T., S.P.): June-July (w.G.).

Antennularia ramosa (Lamouroux): T. Hincks, Hist. Brit. Hydr. Zooph., p. 282, fig.

With $A$. antennina, but less abundant (E.J.A., R.A.T., S.P.).

Breeding: Apr. (R.A.T.): May (w.G., R.A.T., S.P.).

Aglaophenia Helleri, Marktanner-Turneretscher: C. C. Nutting, Journ. Mar. Biol. Assoc., ser. 2, vol. iv, p. 153.

Eddystone Rk. (C.C.N., E.T.B.). 
[ Leptomedusæ-contd. ]

Aglaophenia myriophyldum (Linnæus): T. Hincks, Hist. Brit. Hydr. Zooph., p. 290, fig.

Moderately common, 15-30 fms., on fine sand and sand with gravel (R.A.T., S.P.) : Eddystone Gds. (E.J.A., R.A.T., G.C.B., S.P.) : RameEddystone Gds. (R A.T., S.P.): Stoke Pt. Gds. (s.P.).

Breeding: Apr.-June (R.A.T.): Aug. (w.G.).

The Aplacophoran Rhopalomenia Aglaophenice is very commonly met with twined round the base of the stem of this form (E.J.A., R.A.T., S.P.).

Aglaophenia pluma (Linnæus): T. Hincks, Hist. Brit. Hydr. Zooph., p. 286, fig.

Common on the fronds of Halidrys siliquosa; Bovisand B.; off Mewstone (G.c.B.): Rame-Eddystone Gds. (R.A.T.): Yealm R. (т.V.H., A.J.S.): Penlee-Rame Gds.; N. of Mewstone (E.J.A.): Millbay Ch.; Queen's Gd.; Wembury B.; occasional specs. (E.J.A., R.A.T.).

Breeding: May (E.J.A., R.A.T.): Aug. (w.G.).

Aglaophenia tubulifera (Hincks): T. Hincks, Hist. Brit. Hydr. Zooph., p. 288 , fig.

Not uncommon, Aug.-Oct.; Wembury B.; off the Mewstone (G.c.B): Eddystone Gds. (E.J.A., E.T.B.): Rame-Eddystone Gds.; Mewstone Ledge (R.A.T.): Bovisand B. (G.с.B.).

Breeding: Sept. (R.A.T.): Oct. (w.G.).

Plumularia Alleni, Nutting: C. C. Nutting, Ann. Mag. Nat. Hist., ser. 7, vol. i, p. 364, fig.

Growing on Antennularia ramosa (c.c.n.).

Bearing gonophores: Apr. or May (c.c.n.).

Plumularia Catharina, Johnston: T. Hincks, Hist. Brit. Hydr. Zooph., p. 299 , fig.

Not uncommon; Duke Rk.; Winter Sh.; off Stoke Pt. (G.c.B.): Queen's Gd., occasionally (T.V.H., R.A.T., s.P.): Eddystone Gds., the most abundant species on both the fine sands on other Hydroids, and on the gravels, where Hincks' green variety is often very abundant on Chatopterus tubes (E.J.A.): Rame-Eddystone Gds., on Chatopterus and Terebellid tubes, Cellaria, Antennularia, Ascidiella scabra, etc. (R.A.T.).

With gonophores: Apr.-May (R.A.T.): Aug. (E.J.A.).

Plumularia echinulata, Lamarck : T. Hincks, Hist. Brit. Hydr. Zooph., p. 302 , fig.

On weed, not very common (G.C.B.): Winter Sh., on Delesseria; Millbay Dock, on the piles; Yealm Est., on Laminaria (E.T.в.).

Plumularia frutescens (Ellis \& Solander): T. Hincks, Hist. Brit. Hydr. Zooph, p. 307, fig.

Eddystone Gds., single colony on shell of Pecten maximus (E.J.A.):

Wembury B. (G.c.B.): Stoke Pt. Gds. (s.P.).

Plumularia halecioides, Alder: T. Hincks, Hist. Brit. Hydr. Zooph, p. 306 , fig.

Parasitic on P. setacea and on Antennularia (C.C.N.). 
[ Leptomedusæ-contd. ]

Plumularia pinnata (Linnæus): T. Hincks, Hist. Brit. Hydr. Zooph., p. 295, fig.

By far the most abundant Plumularian at Plymouth (c.c.N.): Millbay Ch. (E.J.A., R.A.T., S.P.): Millbay Dock, on piles; Asia Sh.; Rum B, occasionally (R.A.T.): common; Duke Rk.; Barn Pool; off Mewstone (G.C.B.) : Rame-Eddystone Gds. (E.T.B. \& R.A.T., s.P.) : Eddystone Gds., generally distributed, particularly on the gravel W. of the Eddystone, where it is usually attached to Chotopterus tubes or to other Hydroids (E.J.A.): Yealm R. (E.J.A., R.A.T.).

Asexual 'stoloniferous' reproduction has been observed in Apr. (c.c.n.). Gonophores in Apr. (w.G., R.A.T.): May (E.J.A., R.A.T.): June (E.J.A.).

Plumularia setacea (Ellis): T. Hincks, Hist. Brit. Hydr. Zooph., p. 296, fig.

Common in the Sound; Hincks' branched variety is very common, generally on Halichondria panicea (G.c.B.): Millbay Ch. and Pit, not urreommon; Millbay Dock, on piles; Tinside, occasionally; Asia Sh., occasionally; Rame-Eddystone Gds., on Chotopterus tubes (R.A.T.): Eddystone Gds., generally on other Hydroids or on Polychæte tubes (E.J.A.).

Breeding: Feb.-June (w.G.).

Plumularia similis, Hincks: T. Hincks, Hist. Brit. Hydr. Zooph., p. 303, tig.

Common; rocks below Lab.; Jennycliff B. (G.C.B.): under the Hoe; Bovisand B.; Yealm R. (т.v.H.): Church Reef, Wembury B., very occasional; Millbay Ch., occasional specimens (R.A.T.).

\section{(MEDUSA STAGE.)}

\section{Eucopidæ.}

Agastra mira (Hartlaub) [Hydroid $=A$. caliculata (Hincks)]: $E . T$. Browne, Proc. Zool. Soc., vol. 1897, p. 832., fig.

A single spec., in the Sound, Aug. 1897 (E.T.B.).

Obelia lucifera : E. Forbes, Monogr. Brit. Naked-eyed Med., p. 52, fig. (as Thaumantias).

Very plentiful, June 1892 (Е.J.B.): by far the most abundant Leptomedusan in the Sound and outside, Sept. 1897 (E.T.B.).

Obelia nigra: E. T. Browne, Proc. Irish Acad., ser. 3, vol. v, p. 721.

Common; 1898, very abundant Apr.-May ; 1899, abundant in June (E.T.B.).

Tiaropsis multicirrata (Sars); E. Haeckel; Syst. Medus., Denkschr. med.-naturw. Ges. Jena., vol, i, p. 179.

Apr. 1895 (E.J.A.).

Euchilota pilosella (Forbes): E. T. Browne, Proc. Zool. Soc., vol. 1896 , p. 484 , fig.

Plymouth (E.T.B., E.J.A.); common every summer (W.G.).

Mitrocomella fulva: E. T. Browne, Bergens Mus. Aarb., vol. 1903, No. 4 , p. 17 , fig.

Single spec., off the Eddystone, May 1898 (Е.т.в.). 
Philatidum buskianum (Gosse): E. T. Browne, Proc. Zool. Soc., vol. 1896 , p. 488 , fig.

Sept. 1893 ; Sept. 1895 ; Sept. 1897 , few specs. nearly always present in townettings; June 1898, only once taken (Е.т.в.).

Phialidium cymbaloideum (van Beneden) [Hydroid ?=Campanulina repens]: E. T. Browne, Proc. Zool. Soc., vol. 1896, p. 491, fig.

Sept. 1893; Sept. 1897; Apr.-May 1898; June-July 1899; never very abundant, but a few specimens generally taken (в.т.в.).

Phialidium temporarium : E. T. Browne, Proc. Zool. Soc., vol. 1896, p. 489.

Nearly always present from the spring until autumn (E.т.в.).

Eutima insignis (Keferstein); E. T. Browne, Proc. Zool. Soc., vol. 1896, p. 492 .

Oct. 1893 (W.G., E.T.B. ${ }^{1}$ ): Sept. 1895, single adult; June 1898, S. of the Mewstone, two specs.; Aug. 1899, single spec. (Е.т.в.).

SAPhenta mirabilis (Wright): E. T. Browne, Proc. Zool. Soc., vol. 1896, p. 493 , fig.

Near the Eddystone, July 1891, some hundreds (J.T.c. ${ }^{1}$ ): off Penlee, at bottom in 9 fms., July 1892 (E.J.B. ${ }^{1}$ ): 1897, single specs. in Aug. and Sept.; Apr.-June 1898, few specs.; June-July 1899, few (E.T.B.).

Octorchis Gegenbauri, Haeckel: E. Haeckel, Syst. Medus., Denkschr. med.-naturw. Ges. Jena, vol. i, p. 197, fig.

Sept. 1895, single spec.; 1899, four specs. in July, one in Aug. (E.T.B.).

Irene pelludida (Will): E. Haeckel, Syst. Medus., Denkschr. med.naturw. Ges. Jena, vol. i, p. 201, fig.

Oct. 1892, several specs. (w.G.); Sept. 1897, single spec. from the Sound, two specs. $3 \mathrm{~m}$. S.W. of the Mewstone, all young stages (E.T.B.).

\section{TRACHYMEDUS㞋.}

\section{Geryonidæ.}

Liriantha appendiculata: E. Forbes, Monogr. Brit. Naked-eyed Med., p. 36, fig. (as Geryonia).

Exceedingly abundant Sept.-Oct., 1893; absent Sept. 1895; scarce and only early stages in the Sound and outside grounds, Sept. 1897 (E.T.B.): Jan. 1896 (E.J.A.).

\section{Solmaridæ.}

Solmaris coronanthe, Haeckel: E. Haeckel, Syst. Medus., Denkschr. med.-naturw. Ges. Jena, vol. i, p. 359, fig.

Plymouth, Sept., 1895 (E.T.B.). 


\section{SIPHONOPHORA.}

\section{Monophyidæ.}

Muggifa atlantica: J. T. Cunningham, Journ. Mar. Biol. Assoc., ser. 2, vol. ii, p. 212 , fig.

First met with $5 \mathrm{~m}$. S. of the Eddystone, afterwards in great abundance close to the Breakwater, and even inside the Sound, Sept. 1891 (J.T.C.): 1892, appeared near end of Aug. (E.J.B.): Sept. 1893 , fairly abundant early part of month; Sept. 1895, exceedingly abundant (E.T.в.): 1895, middle Aug.-mid. Dec. (т.v.H.): 1903, occasionally met with in Feb. (w.G.).

\section{Agalmidæ.}

Agalmopsis Sarsi, Haeckel: E. T. Browne, Proc. Irish Acad., ser. 3, vol. v, p. 678 .

Mar., 1902, single specimen from $\frac{1}{2} \mathrm{~m}$. S.W. of the Mewstone and from West Channel (R.A.T.).

\section{STAUROMEDUSR.}

\section{Lucernariidæ.}

Lucernaria Campanulata, Lamouroux: E. Haeckel, Syst. Medus., Denkschr. med.-naturw. Ges. Jena, vol. i, p. 392.

Reny Rks., several small specs. with Haliclystus; single specs. from Cawsand B. and Whitsand B. (w.I.B.).

Haliclystus auricula (Fabricius): W. I. Beaumont, Proc. Irish Acad, ser. 3, vol. v, p. 806.

Yealm Est., on Zostera, fairly common (s.P.): Reny Rks., on Enteromorpha, in high tide rock-pools (A.J.S.): Cawsand B., on Zostera, occasionally (S.P.).

Depastrum cyathiforme: M. Sars, Fauna Litt. Norvegiæ, pt. i, p. 26, fig. (as Lucernaria).

Mount Edgcumbe; Batten (E.J.A.) : Drake's I. ; Rame Hd. (w.G.).

\section{DISCOMEDUS尼.}

\section{Pelagiidæ.}

Chrysaora isosceles (Linnæus): E. Haeckel, Syst. Medus., Denkschr. med.-naturw. Ges. Jena, vol. i, p. 513.

Occasionally during the summer months (s.P.).

Planulæ liberated in Aug. and reared to Scyphistoma stage (E.T.B.).

\section{Cyaneidæ.}

Cyanea capillata (Linnæus): E. Haeckel, Syst. Medus., Denkschr. med.naturw. Ges. Jena, vol. i, p. 529.

Plymouth (E.T.B.).

Cyanea Lamarcki, Péron \& Lesueur: E. Haeckel, Syst. Medus., Denkschr. med.-naturw. Ges. Jena, vol. i, p. 530.

Occasionally, every summer (A.J.S.). 


\section{Ulmaridæ.}

Aurelia Aurita, Lamarck: E. Haeckel, Syst. Medus., Denkschr. med.naturw. Ges. Jena, vol. i, p. 552.

Most abundant in the estuaries in spring and summer; R. Tamar; Hamoaze; Yealm R.; carried out into the Channel towards the end of the summer (E.J.A.): Saltash (E.T.B.).

The ephyræ appear in February and may be taken in shoals during the first fortnight of March, they metamorphose towards the end of the month and begin to disappear. The young medusæ reappear towards the end of May and reach their maximum abundance during June (w.G.).

\section{Pilemidæ.}

Rhizostoma octopus (Linnæus): E. Haeckel, Syst. Medus., Denkschr. med.-naturw. Ges. Jena, vol. i, p. 593 (as Pilema).

Occasionally (S.P.).

\section{ALCYONARIA.}

\section{Cornulariidæ.}

Sarcodictyon catenata, Forbes: W. A. Herdman, Proc. Liverp. Biol. Soc., vol. 1x, p. 163, fig.

Eddystone Gds.; the red form is found, often in abundance, on old shells, and is generally most plentiful on clean shell-gravel (E.J.A., S P.) : Stoke Pt. Gds. ; Rame-Eddystone Gds. ; etc. (s.P.).

\section{Alcyonidæ.}

Alcyonium digitatum, Linnæus: S. J. Hickson, Quart. Journ. Micr. Sci., ser. 2, vol. xxxvii, p. 349, fig.

Generally present in dredgings from the Sound and outside grounds, but the colonies are usually small (R.A.T., S.P.): Promenade Pier, large colonies are occasionally common, at extreme low water (E.J.A., R.A.T., S.P.): Eddystone Gds., large colonies abundant on the fine sand of the 'Outer' Trawling Gds. and S. of the Eddystone, attached to valves of Cardium echinatum, etc. (E.J.A.).

Breeding: Oct.; Jan. (R.A.T.); Nov.-Feb. (w.G.).

Alcyonium glomeratum (Hassall): S. J. Hickson, Quart. Journ. Micr. Sci., ser. 2, vol. xxxvii, p. 353, fig.

Mewstone Ledge (R.A.T.)

Alcyonium Palmatum, Pallas: G. v. Koch, Mitth. Stat. Neapel, vol, ix, p. 663 , fig.

Mewstone Ledge (s.P.): 3 m. S.E. of the Mewstone (E.J.A.).

\section{Plexauridæ.}

Eunicella Cavolini: G. v. Koch, Fauna Flora Neapel, vol, xv, p. 58, fig.

More or less common everywhere on rocky ground, 10-25 fms. (s.P.): Mewstone Ledge, very common (E.J.A., R.A.T., S.P.): Queen's Gd., rare (R.A.T., S.P.): Rame-Eddystone Gds. ; Stoke Pt. Gds. (s.P.).

\section{Virgulariidæ.}

Virgularia mirabilis, O. F. Müller: A. M. Marshall \& W. P. Marshall, Rept. Pennatulida Oban, p. 51, fig.

Single spec., Nr. Eddystone (W.P.M.): $6 \mathrm{~m}$. W.S.W. of Penlee Pt. (R.A.T. ${ }^{1}$ ): Stoke Pt. Gds. (S.P.). 


\section{ZOANTHARIA.}

\section{Cerianthidæ.}

Cerianthus Lloydi, Gosse: P. H. Gosse, Hist. Brit. Sea-Anem., p. 268, fig.

The adult form has only once been taken, in muddy sand on the N. side of Drake's I., but the free-swimming larva (Arachnactis albida) is common during April (R.A.T.).

\section{Zoanthidæ.}

Epizonanthus Couchi (Johnston): A. C. Haddon \& Shackleton, Trans. Dublin Soc., ser. 2, vol. iv, p. 644, fig.

Duke Rk., common (w.G.): Millbay Ch., not uncommon (w.G., R.A.T.).

Epizoanthus incrustatus, Düben \& Koren : A. C. Haddon \& Shackleton, Trans. Dublin Soc., ser. 2, vol. iv, p. 636, fig.

Mewstone Gds., common on shells inhabited by Anapagurus laevis (R.A.T.) : Eddystone Gds., a characteristic species of, and confined to, the 'Outer' Trawling Gds. (E.J.A.).

\section{Edwardsiidæ.}

Edwardsia Beautempsi, Quatrefages: A. C. Haddon, Trans. Dublin Soc., ser. 2, vol. iv, p. 327, fig.

Yealm Sand-bank, single spec. (R.A.T.).

Edwardsia carnea, Gosse: P. H. Gosse, Hist. Brit. Sea-Anem., p. 259, fig. Millbay Ch. (S.P.): Church Reef, Wembury B. (R.A.T.).

\section{Ilyanthidæ.}

Halcampa chrysanthellum (Peach): A. C. Haddon, Proc. Dublin Soc., ser. 2, vol. v, p. 1, fig.

Yealm Sand-bank, common (A.J.S., R.A.T.).

The larvæ parasitic on Medusæ (Irene, Phialidium, etc.), are common in May (w.G., E.J.A., R.A.T.).

Eloactis Mazeli (Jourdan): W. Garstang, Trans. Devon. Assoc., vol. xxiv, p. 380 .

Single spec., few miles off the Mewstone, 20 fms. (w.G.).

\section{Actiniidæ.}

Actinia equina, Linnæus: P. H. Gosse, Hist. Brit. Sea-Anem., p. 175, fig. (as $A$. mesembryanthemum).

Common on rocks between tide-marks (R.A.T., S.P.).

Breeding: Jan.-Aug. (R.A.T.).

Anemonia sulcata (Pennant): P. H. Gosse, Hist. Brit. Sea-Anem., p. 160, fig. (as Anthea cereus).

More or less common everywhere on rocks between tide-marks and on the Zostera beds. The slate-coloured variety is more abundant than the typical form with violet-tipped, green tentacles, and on the Zostera it is alone present. A flesh-coloured variety is occasionally met with. Yealm Sand-bank; brownish var. only (S.P.). 
[Zoantharia-contd. ]

Metridium senilis (Linnæus): P. H. Gosse, Hist. Brit. Sea-Anem., p. 12, fig. (as Actinoloba dianthus).

Promenade Pier, very common on the piles at extreme low water (R.A.T., S.P.): Millbay Ch., occasional specimens (R.A.T.): Yealm Sandbank, occasionally (R.A.T.): Yealm Estuary, young specs, common on the rocks between tide-marks (S.P.).

Breeding, in tanks, Aug.-Sept. (s.P.).

Sagartia miniata (Gosse): P. H. Gosse, Hist. Brit. Sea-Anem., p. 41, fig. Asia Sh. (A.J.S., R.A.T.): Millbay Ch. (R.A.T.).

Sagartia nivea (Gosse): P. H. Gosse, Hist. Brit. Sea-Anem., p. 67, fig. Rocks below the Laboratory (G.C.B. ${ }^{1}$ ).

Cereus pedunculatus (Pennant): $P$. $H$. Gosse, Hist. Brit. Sea-Anem., p. 27, fig. (as Sagartia bellis).

Common on the shore where the ground is suitable, this form seeming to prefer muddy sand with stone. Not uncommon in dredgings from Millbay Ch., Mallard Sh., etc. (R.A.T.). Especially common in the estuaries of the Yealm, Tamar, and Plym (E.J.A.).

Breeding: Jan.-Feb.; Dec. (w.G.).

Chondractinia digitata (O. F. Müller): A. C. Haddon, Trans. Dublin Soc., ser. 2, vol. iv, p. 306, fig.

Eddystone Gds., few specimens on the 'outer' trawling grounds, and on the fine sand S. of the Eddystone, inside valves of Cardium echinatum (E.J.A.).

Chitonactis coronata (Gosse): $P$. H. Gosse, Hist. Brit. Sea-Anem., p. 202, fig. (as Bunodes).

Occasionally in the deeper water outside the Breakwater, and in Millbay Ch. (w.G.): Rame-Eddystone Gds., single spec. on Hydroid stem (R.A.T.).

Breeding: Jan.-Apr. (w.G.).

Paraphellia expansa (Haddon): A. C. Haddon, Trans. Dublin Soc., ser. 2, vol. iv, p. 321, fig.

Eddystone Gds., few specimens, apparently living buried in the sand (E.J.A.): Rame-Eddystone Gds. (R.A.T.).

Cylista undata (O. F. Müller): P. H. Gosse, Hist. Brit. Sea-Anem., p. 88, fig. (as Sagartia troglodytes).

Common at Plymouth (w.G.).

Cylista viduata (O. F. Müller): $P$. H. Gosse, Hist. Brit. Sea-Anem., p. 105, fig. (as Sagartia).

Rare within the Sound, but common in the neighbourhood (w.G.) : Promenade Pier, not uncommon on the piles at extreme low tide (R.A.T., A.J.S.) : Millbay Ch., not uncommon (R.A.T.).

Adamsia palliata (Bohadsch): P. H. Gosse, Hist. Brit. Sea-Anem., p. 125 , fig.

Generally distributed and common, 10-30 fms., associated with Eupagurus Prideauxi. Eddystone Gds., Rame-Eddystone Gds., Mewstone Gds., Cawsand B,, Yealm R., ete. (s.P.). 
[ Zoantharia-contd. ]

Adamsia polypus (Forskal): P. H. Gosse, Hist. Brit. Sea-Anem., p. 112, fig. (as Sagartia parasitica).

More or less common everywhere 15-30 fms., associated with Eupagurus Bernhardus (S.P.).

Aiptasia Couchi (Cocks): P. H. Gosse, Hist. Brit. Sea-Anem., p. 152, fig. Rocks below the Lab., occasionally (G.C.B., R.A.T.) : Reny Rks., not uncommon (R.A.T.).

Thoe sphyrodeta (Gosse): P. H. Gosse, Hist. Brit. Sea-Anem., p. 73, fig. (as Sagartia).

Drake's I., at low tide (w.H.): Mallard Sh. (J.c.s.): Millbay Ch., occasionally (R.A.T., E.J.A.).

Bunodes Balli (Cocks): P. H. Gosse, Hist. Brit. Sea-Anem., p. 198, fig.

The Breakwater; the typical form is rare, being replaced by Gosse's varieties "dealbata" and "livida" (w.G.).

Bunodes gemmacea, Ellis : P. H. Gosse, Hist. Brit. Sea-Anem., p. 190, fig.

Caves under the Lab. (G.C.B.): Drake's I., common on rocks (E.J.A., R.A.T., S.P.) : Mt. Edgeumbe (E.J.A.): Rum B., not uncommon between tide-marks (R.A.T., E.J.A.): Jennycliff B., between tide-marks, rare (R.A.T.): Bovisand Cove (R.A.T.): Whitsand B., common between tide-marks (R.A.T.).

Breeding: Mar. (R.A.t.) : Apr.; Sept. (w.G.).

\section{Corallimorphidæ.}

Corynactis viridis, Allman: P. H. Gosse, Hist. Brit. Sea-Anem., p. 289, fig.

More or less common in crevices and under stones on all rocky stations, L.W.-15 fms. (S.P.): The Breakwater (E.J.A., R.A.T., S.P.): Millbay Ch. (R.A.T., S.P.).

\section{Amphianthidæ.}

Gephyra Dohrni, v. Koch: A. C. Haddon, Trans. Dublin Soc., ser. 2, vol. iv, p. 325, fig.

Mewstone Ledge, on Eunicella, not uncommon (T.V.H., A.J.S., R.A.T., S.P.).

\section{Urticinidæ.}

Urticina felina (Linnæus): P. H. Gosse, Hist. Brit. Sea-Anem., p. 209, fig. (as Tealia crassicornis).

Not uncommon on rocks between tide-marks and occasionally in 10-20 fms. in the Sound. The specimens are not usually of great size, but large ones are occasionally trawled in 20-40 fms. (R.A.T.).

Breeding: May (w.G.).

\section{Turbinolidæ.}

Caryophyllia Smithi, Stokes: $P$. H. Gosse, Hist. Brit. Sea-Anem., p. 310 , fig.

More or less abundant on all rocky stations, L.W.-30 fms., under boulders and in rock-crevices (S.P.): The Breakwater; Mewstone Ledge, etc. (E.J.A., S.P.).

The Cirripede Pyrgoma anglicum is commonly found attached to the margin of the cup of this species; and frequently several occur upon a single coral (s.P.). 
[Ctenophora: Echinoderma]

\section{CTENOPHORA.}

Bolina infundibulum (Fabricius): E. Vanhöffen, Nordisches Plankt., pt. xi, p. 5, fig.

Abundant in May of particular years (w.G.): Sept,, 1900 (A.J.S.).

Beroe cucumis, Fabricius: E. Vanhöffen, Nordisches Plankt., pt. xi,

p. 7, fig.

Plymouth, few small specs. (E.T.B.).

Pleurobrachia pileus (Fabricius): E. Vanhöffen, Nordisches Plankt., pt. xi, p. 3, fig.

Always abundant towards the end of May (w.G.). Adults not seen after June; minute specs, appeared in Sept. (E.J.B.): Aug. (T.V.H.).

\section{ECHINODERMA.}

Synaptidæ.

Synapta digitata (Montagu): F. J. Bell, Catal. Brit. Echinod. Brit. Mus., p. 34, fig.

Rame-Eddystone Gds., occasional specs. (R.A.T.).

Synapta inharens (O. F. Müller): $F$. J. Bell, Catal. Brit. Echinod. Brit. Mus., p. 33, fig.

Drake's I., occasional specs.; Yealm R., not uncommon between tide-marks in coarse, loose sand (R.A.T.).

\section{Cucumariidæ.}

Cucumaria Brunnea (Thompson): E. Forbes, Hist. Brit. Starf., p. 229, fig. (as Ocnus).

Frequently in some numbers on Hydroids, Algæ, etc., from rocky ground and from the trawling grounds; below tide-marks to $25 \mathrm{fms}$. (s.P.): often abundant on rocky ground off the Mewstone (E.J.A.): Yealm R., not uncommon (R.A.T.).

Cucumaria Hyndmani (Thompson): This species may be readily recognised by the silvery appearance of its test.

Occasional specs. not uncommon on coarse ground; Stoke Pt. Gds.; Rame-Eddystone Gds.; Looe-Eddystone Gds. (s.P.).

Cucumaria Normani*: S. Pace, see p. 309.

Not uncommon on rocky ground in crevices and under stones, L.W.-10 fms. (s.P.).

Cucumaria saxicola $\dagger$, Brady \& Robertson: S. Pace, see p. 306.

Not uncommon on rocky ground, in crevices and under stones, L.W.-10 fms. (s.P.).

Thyone fusus (O. F. Müller): F. J. Bell, Catal. Brit. Echinod. Brit. Mus., p. 42, fig.

Occasional specs. from Millbay Ch., Cattewater, Cawsand B.; not uncommon on the Mewstone Gds., and occasionally met with in 15-35 fms. on other grounds (R.A.T.).

\footnotetext{
* Previously recorded as C. Planci.

+ Previonsly recorded as $C$. pentactes.
} 


\section{Holothuriidæ.}

Holothuria nigra, Gray: F. J. Bell, Catal. Brit. Echinod. Brit. Mus., p. 49 , fig.

Common on the Mewstone Gds. on clean gravel (E.J.A., R.A.T., S.P.): occasional specs. have been taken in Whitsand B., off Penlee, Melampus Sh. (R.A.T.): Stoke Pt. Gds., not uncommon (s.P.): Eddystone Gds., on the clean shell gravel immediately adjacent to the rocks (E.J.A.): Queen's Gd. (S.P.).

Gonads ripe: Apr. (s.P.): July, Dec. (R.A.T.).

\section{Antedonidæ.}

Antedon bifida (Pennant) [=A. rosacea, auctt.]: F. J. Bell, Catal. Brit. Echinod. Brit. Mus., p. 54, fig.

Extremely abundant at certain small areas, but practically restricted to these (S.P.): Millbay Pit (R.A.T., S.P.): Asia Sh., occasionally (R.A.T., S.P.): Mallard Sh. (G.C.B., J.T.C., S.P.): inside the Bridge (s.P.): the Cattewater, several specs. on bottom of coal hulk off Turnchapel (A.J.S., S.P.): Mewstone Ledge (s.P., R.A.T.).

The marsupia containing ova: Oct. (т.v.H.). Pentacrinoid larvæ in Feb. (A.J.S.): July (R.A.T., S.P.): Aug., abundant at all stages, chiefly on Cellaria (E.w.L.H.): Sept.-Oct. (w.G.).

The parasitic Polychæte Myzostoma cirriferum is common on the arms of this species (E.J.A.).

\section{Astropectinidæ.}

Astropecten irregularis (Pennant): E. Forbes, Hist. Brit. Starf., p. 130, fig. (as Asterias aurantiacus).

Common on fine, clean sand and moderately so on clean gravel and shell, 15-35 fms. (R.A.T., S.P.): Eddystone Gds. (E.J.A., S.P.): Mewstone Gds. (R.A.T., S.P.): Stoke Pt. Gds.; Rame-Eddystone Gds.; Looe-Eddystone Gds. (s.P.).

Luidia Sarsi, Düben \& Koren: F. J. Bell, Catal. Brit. Echinod. Brit. Mus., p. 72 .

Eddystone Gds. (E.J.A., J.T.C., R.A.T.).

\section{Gymnasteriidæ.}

Porania pulvillus (O. F. Müller): F. J. Bell, Catal. Brit. Echinod. Brit. Mus., p. 79, fig.

Occasionally at about $3 \mathrm{~m}$. S. of the Breakwater (E.J.A., S.P.): Eddystone Gds. (E.J.A., R.A.T.).

Breeding: Feb.-Apr. (R.A.T.).

\section{Asterinidæ.}

Asterina gibbosa (Pennant): $F$. $J$. Bell, Catal. Brit. Echinod. Brit. Mus., p. 82, fig.

Common under stones at all rocky shore stations (S.P.): single spec. $\frac{1}{2} \mathrm{~m}$. S. of the Breakwater in 7 fms. (R.A.T.).

Breeding: May-June (w.G.).

Palmipes Placenta (Pennant): E. Forbes, Hist. Brit. Starf., p. 116, fig. (as P. membranaceus).

Eddystone Gds., moderately common (E.J.A., S.P.): Rame-Eddystone Gds. (R.A.T., S.P.): Stoke Pt. Gds., not uncommon; about $3 \mathrm{~m}$. S. of Breakwater, fairly common (s.P.). 


\section{Solasteridæ.}

Solaster papposus (Fabricius): E. Forbes, Hist. Brit. Starf., p. 112, fig.

Fairly common on coarse sand and gravel, $15-35$ fms. (s.P.): the most plentiful starfish in 1892 (w.G.): Mewstone Gds., RameEddystone Gds. (R.A.T., S.P.): Eddystone Gds. (E.J.A., S.P.): Stoke Pt. Gds. (s.P.): Cattewater, probably from trawl refuse (R.A.T., S.P.). Gonads ripe in Mar. (т.V.H.).

\section{Echinasteridæ.}

Henricia sanguinolenta (O. F. Müller): E. Forbes, Hist. Brit. Starf., p. 100, fig. (as Cribella oculata).

Reny Rks., occasional specs. at extreme low water (R.A.T.): not uncommon on the Mewstone Gds. (R.A.T., S.P.): Eddystone Gds., occasionally on clean medium gravel (E.J.A., S.P.): Stoke Pt. Gds. (s.P.).

\section{Asteriidæ.}

Asterias glacialis, Linnæus: H. Ludwig, Fauna Flora Neapel, vol. xxiv, p. 364, fig.

More or less common at all stations below low-water mark, but somewhat uncertain in its occurrence (s.P.): large specs. generally from deeper water, $30 \mathrm{fms}$. and over (E.J.A.): the Breakwater, not uncommon; Yealm R., not uncommon in dredgings, large specs. occasionally on the Sand-bank (R.A.T.): Eddystone Gds., most abundant where Pecten opercularis is plentiful (E.J.A.).

Asterias rubens, Linnæus: E. Forbes, Hist. Brit. Starf., p. 83, fig. (as Uraster).

Occurs below tide-marks at most stations, but in very varying abundance in different years; it is generally plentiful, but occasionally it would seem to be almost absent; * a smaller, violet-coloured var. is met with on coarse ground in $15-30$ fms. (E.J.A., S.P.).

\section{Ophiolepidæ.}

Ophiura albida, Forbes: F. J. Bell, Catal. Brit. Echinod. Brit. Mus., p. 108.

Lives generally on coarser soil and is less abundant than 0 . ciliaris (E.J.A.): Mewstone Gds. (R.A.T.): Stoke Pt. Gds., fairly common; Rame-Eddystone Gds. (s.P.): Eddystone Gds. (E.J.A., S.P.).

Ophiura ciliaris (Linnæus): $F$. J. Bell, Catal. Brit. Echinod. Brit. Mus., p. 106.

Common, 15-35 fms., on gravel and sand (s.P.): most frequent on fine hard sand (E.J.A.): Mewstone Gds. (R.A.T., s.P.): Stoke Pt. Gds. (S.P.): Rame-Eddystone Gds. (E.J.A., R.A.T., S.P.): Eddystone Gds. (E.J.A., S.P.): Looe-Eddystone Gds. (s.P.).

Breeding: May (R.A.T.): Aug. (s.P.).

* Not a single spec. of $A$. rubens was obtainable from towards the end of Oct. 1903 until well on in the spring of 1904 , although repeated search was made for it both in the Sound and in Cawsand B. (s.P.). 


\section{Amphiuridæ.}

Ophiocnida brachiata (Montagu): F. J. Bell, Catal. Brit. Echinod. Brit. Mus., p. 116, fig.

Common in muddy sand on a Zostera bed S. of Batten Castle (E.J.A., R.A.T., S.P.) : Jennycliff B., single spec. (R.A.T.).

Amphiura Chiajir, Forbes: $F$. J. Bell, Catal. Brit. Echinod. Brit. Mus., p. 117: two mouth papillæ on each side, two tentacle scales, 5-8 arm spines.

Millbay Ch., single spec. (R.A.T.): 2 m. S. of the Breakwater, single spec. (E.J.A.).

Amphiura elegans (Leach): F. J. Bell, Catal. Brit. Echinod. Brit. Mus.,

p. 119: three mouth papillæ on each side, two tentacle scales.

Common at most stations in the Sound, L.W.-20 fms. (s.P.):

Rame-Eddystone Gds., 27 fms., single spec. (R.A.T.): Yealm R. (s.P.). Breeding: May-Sept. (w.G.).

Amphiura filiformis (O. F. Müller): $F$. J. Bell, Catal. Brit. Echinod. Brit. Mus., p. 119: two mouth papillæ on each side, no tentacle scales, arms very long.

Single spec., 2 m. W.S.W. of the Eddystone (E.J.A.).

Ophiactis Balli (Thompson): F. J. Bell, Catal. Brit. Echinod. Brit. Mus., p. 124.

Common, but rather local, 15-35 fms., in crevices of rock and stones and on Choetopterus tubes (s.P.): Millbay Ch. and Pit, not uncommon (R.A.T., S.P.): Asia Sh. (T.V.H., S.P.): Mewstone Gds.; Rame-Eddystone Gds. (R.A.T., S.P.) : abundant around the Eddystone (E.J.A.): Stoke Pt. Gds., not uncommon (s.P.).

Gonads ripe: Sept. (s.P.).

\section{Ophiocomidæ.}

Ophiocoma nigra (O. F. Müller): E. Forbes, Hist. Brit. Starf., p. 50, fig. (as 0 . granulata).

This form is almost invariably found associated with Ophiothrix fragilis, but is generally far less abundant than the latter species, although occasionally it is the predominant form; at most stations the specimens are brilliantly coloured, but sometimes, as on the Mallard Sh., it is possible to get a pure gathering of the typical black form (S.P.): Mallard Sh., in abundance together with Antedon (w.G., s.P.): Millbay Pit, common; Mewstone 'Echinoderm' Gd. (R.A.T., S.P.): Eddystone Gds., occasional specs. on stony and rocky ground, 15-30 fms. (E.J.A., S.P.): Rame-Eddystone Gds.; Stoke Pt. Gds. ; not uncommon but very local (s.P.).

Gonads ripe: Apr.; July. Spent individuals, Sept. (s.P.).

Ophiopsila aranea, Forbes: A. E. Grube, Die Insel Lussin (Breslau, 1864), p. 104.

Mewstone Ledge, not uncommon in rock crevices (E.J.A., R.A.T., S.P.): Stoke Pt. Gds., fairly common in crevices of the local red rock, especially in old Plioladidea crypts, $15-25$ fms. (s.P.).

Gonads ripe in Aug. (R.A.T.). 
[ Echinoderma-cond. ]

\section{Ophiothricidæ.}

Ophiothrix fragilis (O. F. Müller): E. Forbes, Hist. Brit. Starf., p. 60, fig. (as Ophiocoma rosula).

Generally distributed and enormously abundant at certain stations, L.W.-35 fms.; a small, solitary, greyish-coloured form occurs under stones on the shore; in deeper water it grows to a much larger size and is usually very brilliantly coloured; this latter form favours a coarse gravel ground and lives in such profusion where it occurs that the dredge will frequently come up completely filled with a practically pure gathering of this species alone (s.P.).

Gonads ripe: Mar.-June (R.A.T.) : Aug.-Sept. (s.P.). Spawning : Oct. (R.A.T.).

\section{Echinidæ.}

Echinus acutus, Lamarck: F. J. Bell, Catal. Brit. Echinod. Brit. Mus., p. 146.

Not uncommon in 15-35 fms., chiefly on the finer grounds (R.A.T., S.P.): more common in deeper water, where it replaces $E$. esculentus (E.J.A.): Mewstone Gds. (R.A.T.): Stoke Pt. Gds. (s.P.): RameEddystone Gds. (R.A.T., S.P.): Eddystone Gds. (E.J.A., S.P.): LooeEddystone Gds. (s.P.).

The Polychæte Siphonostoma affinis is often found associated with this form, crawling among its spines (R.A.T.).

Breeding in July (w.G.).

Echinus esculentus, Linnæus: A. Agassiz, Illustr. Catal. Mus. Harvard, No. vii, p. 491, fig.

Queen's Gd., occasionally; moderately common in 15-35 fms., especially on the Mewstone 'Echinoderm' Gd.; Rame-Eddystone Gds. (R.A.T., S.P.): Eddystone Gds. (E.J.A., S.P.): Mewstone Ledge; Stoke Pt. Gds., common (s.P.): Cattewater, probably from trawl refuse (R A.T., S.P.).

The Polychrte Scalesetosus assimilis is often found among the oral spines of this species (R.A.T.).

Gonads ripe: Mar. (R.A.T.): May (s.P.).

Echinus miliaris, Linnæus : $E$. Forbes, Hist. Brit. Starf., p. 161, fig.

Common under stones at all shore stations, and frequently met with in dredgings, L.W.-35 fms. (s.P.): specs. from the deeper water stations are usually small; largest ones from Church Reef, Wembury B. (R.A.T.): Yealm R., very common near the oyster beds (E.J.A.).

Breeding: May (w.G.).

\section{Clypeastridæ.}

Echinocyamus pusillus (O. F. Müller): F. J. Bell, Catal. Brit. Echinod. Brit. Mus., p. 160, fig.

Not uncommon on gravel bottom, 10-35 fms. (s.P.): Queen's Gd., occasionally (R.A.T., S.P.): Mewstone Ledge shell gravel, very occasionally; Mewstone 'Amphioxus' Gd., moderately common (R.A.T.): Eddystone Gds. (E.J.A.). 


\section{Spatangidæ.}

Spatangus purpureus, O. F. Müller: F. J. Bell, Catal. Brit. Echinod. Brit. Mus., p. 165.

Moderately common in coarse sand and gravel, $15-35$ fms. (s.P.): Eddystone Gds. (E.J.A., S.P.): Stoke Pt. Gds.; Rame-Eddystone Gds. (R.A.T., S.P.): Looe-Eddystone Gds. (S.P.): Mewstone Ledge (R.A.T.) : Queen's Gd. (s.P. $\left.{ }^{1}\right)$.

The Molluse Montacuta substriata is very commonly attached to the oral spines of this species (R.A.T., S.P.).

Echinocardium cordatum (Pennant): E. Forbes, Hist. Brit. Starf., p. 190, fig. (as Amphidotus): anterior ambulacrum in a deep groove.

Not uncommon buried in sand between tide-marks in the Yealm Estuary; and in Rum B., on the Zostera beds; occasionally dredged in Cawsand B., and outside in 15-35 fms. on fine sand (R.A.T.): Rame-Eddystone Gds. (R.A.T., S.P.): Eddystone Gds. (E.J.A., s.P.): Looe-Eddystone Gds.; common $6 \mathrm{~m}$. S. of Breakwater (s.P.).

The Mollusc Tellimya ferruginosa is found associated with this species (R.A.T.).

Echinocardium Pennatifidum, ${ }^{*}$ Norman: F. J. Bell, Catal. Brit. Echinod. Brit. Mus., p. 170, fig.: anterior ambulacrum not in a groove, postero-lateral ambulacrum with generally 14 and 12 pores.

Not uncommon 15-30 fms.; Rame-Eddystone Gds.; Stoke Pt. Gds.; Looe-Eddystone Gds. (s.P.).

Echinocardium flavescens (O. F. Müller): $F$. J. Bell, Catal. Brit. Echinod. Brit. Mus., p. 171, fig.: anterior ambulacrum not in a groove, postero-lateral ambulacrum with generally 7 and 6 pores, spines thick-set and silky.

Looe-Eddystone Gds., rare, associated with $E$. pennatifidum, but its habitat is probably on finer ground than that of the latter species (S.P.).

\section{TURBELLARIA.}

(Nomenclature that of F. W. Gamble, British Marine Turbellaria. Quart. Journ. Micr. Sci., xxxiv, 1893.)

\section{Proporidæ.}

Proporus venenosus (O. Schmidt).

Not uncommon at low water Wembury B. and Drake's I. (F.w.G.). Monoporus rubropunctatus (O. Schmidt).

Not uncommon at low water, Wembury B. and Drake's I. (F.w.G.).

\section{Aphanostomidæ.}

Aphanostoma Diversicolor, Oersted.

Various localities between tide-marks (F.w.G.).

Aphanostoma elegans, Jensen.

One specimen amongst Ulva at Redding Pt. (F.W.G.).

Convoluta saliens, v. Graff.

Among Zostera from Cawsand B., rare (F.w.G.).

* The specimen recorded, Journ. Mar. Biol. Assoc., ser. 2, vol. v, p. 530, as E. pennatifidum, was really E. flavescens (E.J.A.). 
Convoluta paradoxa, Oersted.

[ Turbellaria-contd. ]

Littoral zone, widely distributed, nowhere abundant (F.w.G.).

Convoluta flavibacillum, Jensen.

Among sand in creeks at Picklecombe Fort, Wembury B. and Bovisand B. (F.w.G.).

\section{Microstomidæ.}

Microstoma Grenlandicum, Lev.

Among Ulva, Redding Pt. (F.w.G.).

\section{Mesostomidæ.}

Promesostoma marmoratum (Schulze).

Not uncommon in tide-pools in Wembury B., Drake's I., and Redding Pt. (F.w.G.).

Promesostoma ovoideum (O. Schmidt).

Occasionally dredged near Duke Rk. (F.w.G.).

Promesostoma solea (O. Schmidt).

Abundant in dredging from all localities (F.w.G.).

Promesostoma agile (Levinsen).

Among Zostera in Cawsand B. (F.w.G.).

Byrsophlebs Graffi, Jensen.

Drake's I., low spring-tide; amongst algæ (F.w.G.).

Brysophlebs intermedia, v. Graff.

Drake's I., low spring-tide; amongst algæ (F.w.G.).

Proxenetes cochlear, v. Graff.

Plymouth Sound (E.G.G.).

Proxenetes flabellifer, Jensen.

Tide-pools on north side of Cawsand B. (F.w.G.).

Mesostoma neapolitanum, v. Graff (?).

One specimen among Fuci on inner side of Breakwater (F.w.G.).

Proboscidæ.

Pseudorhynchus bifidus (MeIntosh).

Plymouth Sound (E.G.G.).

ACrorhynchus Caledonicus (Claparède).

Tide-pools near Picklecombe and Redding Pt.; less commonly in Wembury B. (F.W.G.).

Macrorhynchus NaEgeli (Kölliker).

Plentiful in August on inner side of Breakwater (F.w.G.).

Macrorhynchus CRoceus (Fabricius).

Dredged once on New Gds. (F.w.G.).

Macrorhynchus helgolandicus (Metschnikoff).

Once on New Gds. (F.w.G.).

GyRATOR HERMAPHRODITUS, Ehrbg.

Tide pools on rocks in front of Laboratory: water sometimes brackish. In early winter, not in spring (E.G.G.).

HyPORHYNCHUS ARMATUS (Jensen).

Among Zostera in Cawsand B.; tide-pools Redding Pt. (F.w.G.).

Tanks in Laboratory (E.G.G.). 
[ Turbellaria-contd. ]

HyPORHYNCHUS PENICILLATUS (Schmidt).

One specimen among Zostera in Cawsand B. (F.w.G.).

Provortex balticus (Schultze).

\section{Vorticidæ.}

Between tide-marks, chiefiy at Wembury B. (F.w.G.).

Provortex AfFinis (Jensen).

Drake's I, among algæ (F.W.G.).

Provortex Rubrobacillus, Gamble.

Dredged off New Gds. (F.W.G.).

Fecampia erythrocephala: A. Giard, C. R. Acad. Paris, vol, ciii, p. 499.

Encysted stage common on all stony shores at low water(w.I.B., S.P.).

\section{Plagiostomidæ.}

Plagiostoma dioicum (Metschnikoff).

Duke Rk. and Wembury B. (F.w.G.).

Plagiostoma elongatum, Gamble.

Wembury B., among sand; Breakwater (F.w.G.).

Plagiostoma pseudomaculatum, Gamble.

Among weed-tubes of Polydora creca in Hamoaze (F.w.G.).

Plagiostoma sagitTa, Uljanin.

Tide-pool Redding Pt. (F.w.G.).

Plagiostoma caudatum, Levinsen.

One specimen among Zostera in Cawsand B. (F.w.G.).

Plagiostoma vittatum (Frey u. Leuckart)

Abundant littoral species in all localities. Egg capsules from Breakwater in September (F.w.G.).

The tanks in the Laboratory swarm with them (E.G.G.).

Plagiostoma Koreni, Jensen.

Breakwater and Redding Pt. Tank in Laboratory (F.W.G.).

? Plagiostoma SipHonophorum (Schmidt).

A specimen from Millbay Ch. (F.w.G.).

Plagiostoma Girardi (Schmidt).

Low spring-tides, Wembury B.; tide-pools north side of Cawsand B.

Not uncommon Duke Rk. and Millbay Ch. (F.w.G.).

Vorticeros auriculatum (O. F. Müller).

An abundant littoral species in all localities (F.w.G.).

Vorticeros LUteum, v. Graff.

One specimen New Gds.; one on inner side of Breakwater (F.w.G.).

Enterostoma austriacum, v. Graff.

Common in Sound below 5 fms. (F.w.G.).

Enterostoma fingalianum, Claparède.

Among Floridea, Wembury B. (F.w.G.).

Cylindrostoma QUADRIOCULATUM, (R. Leuckart).

Abundant among Florida, Wembury B. (F.w.G.).

Tanks in Laboratory (E.G.G.).

Cylindrostoma inerme (Hallez).

Duke Rk., Millbay Ch., Hamoaze (F.w.G.). 
[ Turbellaria-contd.]

Cylindrostoma elongatum, Levinsen.

Tide-pools, Wembury B. (F.w.G.).

MoNOOPHORUM STRIATUM (v. Graff).

Duke Rk., a single specimen (F.w.G.)

\section{Monotidæ.}

Monotus lineatus (O. F. Müller).

Not uncommon amongst Ulva, Redding Pt. (F.W.G.).

Monotus fuscus (Oersted).

Abundant among Balani, Ulva, and generally throughout the littoral zone (F.W.G.).

MoNotus albus, Levinsen.

Tide-pools below Picklecombe Fort (F.w.G.).

Automolos unipunctatus (Oersted).

Rarely among algæ, Duke Rk. (F.W.G.).

Automolos HORRIDUs, Gamble.

One specimen from Hamoaze (F.W.G.).

(?) Automolos ophiocephalus (Schmidt).

Millbay Ch. (F.W.G.).

Fovia AFFinis, Stimpson.

Planariidæ.

In a sandy creek, Wembury B. (F.W.G.): Cawsand B.; Whitsand B. ; on drift weed (w.I.B.).

Cryptocelis alba, Lang.

\section{Leptoplanidæ.}

Millbay Ch.; Mewstone Amphioxus Gd., Nov., 1899 (w.I.B.). Confirmed F. W. Gamble.

LEPTOPLANa TREMELLARIS (O. F. Müller).

Generally, under stones and shells, from littoral zone to $15 \mathrm{fms}$. (F.W.G., W.I.B.) : plentiful in July and August, searcer in September. Difficult to find in February (F.W.G.): Millbay Pit; about 2 m. S. of Mewstone; Yealm R. (w.I.B.).

LEPTOPLANA DRABACHENSIS, Oersted.

Plymouth Sound (F.W.G.).

LEPTOPLANA FALLAX (Quatrefages).

Millbay Ch. (F.W.G.).

\section{Planoceridæ.}

Stylochoplana maculata, Quatrefages.

Cawsand B., common on trawled weed, July, 1898 (w.I.B.).

\section{Euryleptidæ.}

Prosthecereus vittatus (Montagu).

Off Stoke Pt. (J.T.C.): Sound (w.G.) [F.w.G.]: Queen's Gd., occasionally; Yealm R., not uncommon, sometimes very large (w.I.B.).

CyCloporus PAPILLOSUS, Lang.

On Ascidians and sponges dredged in Cattewater and outside Sound (F.w.G.): on Botryllus under stones below the Laboratory (E.J.A.): Barn Pool, var. laevigatus; Mt. Edgeumbe; on Fucus with Botrylloids; Queen's Gd.; Duke Rk. (w.I.B.): common on Botryllus (S.P.). 
[ Turbellaria-contd. : Nemertini]

Eurylepta cornuta (O. F. Müller).

Occasionally dredged on Duke Rk. and in Yealm R. (F.w.G.): Drake's I., N. shore; Yealm R.; off the Mewstone, on gravel and rough ground; Rame-Eddystone Gds. (w.I.B.): Asia Sh.; Queen's Gd.; etc. (s.P.).

Oligocladus SANGUINOLENTUS (Quatrefages).

Duke Rk.; Millbay Ch.; Cawsand B.; Stoke Pt. (F.w.G.): Queen's Gd.; Mallard Sh.; Mewstone 'Cellaria' and 'Echinoderm' Gds.; Yealm R., common (w.I.B.).

Stylostomum variabile, Lang.

Estuary of Yealm, Duke Rk. Young stages between tide-marks at Redding Pt. and round Mallard Buoy in September (F.w.G.) : not uncommon on stony bottom in the Sound and Yealm R.; Cawsand B., on drift weed; Millbay Docks, on Piles with Ascidiella (w.I.B.).

\section{Prosthiostomidæ.}

Prosthiostomum siphunculus (Delle Chiaje): A. Lang, Die Polycladen d. Golf. Neapel, p. 595, fig.

Drake's I. (F.W.G.).

\section{NEMERTINI.}

Nomenclature :-Bürger. Nemertinen des Golfes von Neapel. 1895. (Unless otherwise stated.)

\section{Carinellidæ.}

Carinella linearis, MeIntosh.

Two specimens Duke Rk. (1892) (T.H.R.).

Carinella polymorpha (Renier).

One specimen, Stoke Pt., 25 fms. 22.3 .92 (т.H.r.): Mewstone Amphioxus ground, 10.9 .95 ; about half-way between Rame and Eddystone, 20.12.28; $4 \mathrm{~m}$. W. of Eddystone, 9.4.00, one specimen on each occasion (w.I.B.).

Carinella superba (Kölliker) (=C. annulata, Montagu of Riches and of Bürger's earlier works).

Six miles S.E. of Mewstone, one specimen (T.H.R.): sand-bank in Yealm (R.C.P., W.I.B.): Drake's I., Mewstone Gds., Rame to Eddystone and Eddystone Gds. (w.I.B.).

Carinella annulata, Montagu ( $=C$. MeIntoshii of Bürger's earlier papers and of Riches $;=C$. aragoi, Joubin).

Not uncommonly dredged 5-20 fms. (T.H.R.): Millbay Ch. (w.I.B., R.A.T.): Queen's Gd., Asia Sh. (W.I.B., R.A.T., A.J.s.): Duke Rk., Yealm R., Mewstone Gds., 6 m. S.W. of Rame: Eddystone Gds. (W.I.B.).

\section{Cephalothricidæ.}

Cephalothrix bioculata, Oersted.

Common between tide-marks, in clean, coarse sand and among corallines (т.H.R.): Drake's I., Rum B., Mt. Edgeumbe (E.J.A., W.I.B.): Millbay Ch. (w.I.B.).

Breeding: Apr. to Aug.; pelagic larvæ as late as Dec. (T.H.R.). 
[ Nemertini-contd. ]

Cephalothrix linearis (Rathke), Oersted.

Rum B. in sand between tide-marks; dredged outside Breakwater (T.H.R.).

Breeding in March (w.G.).

\section{Eunemertidæ.}

Eunemertes Neesi (Oersted).

Abundant on Breakwater, rare elsewhere (т.н.R.): the Bridge, Drake's I., common (R.A.T., W.I.B.): Wembury B. (E.J.A., T.V.H.).

Breeding: Feb. (R.A.T.) : March to Oct. (T.H.R.).

EUNEMERTES GRACILIS, Johnston.

Breakwater, among Laminaria roots (T.H.R.): Drake's I. (R.A.T., W.I.B.).

EUNEMERTES ECHINODERMa (Marion).

Yealm, one specimen dredged 25.1.99, one from sand-bank 1.2.00 (w.I.B.): Yealm at low water (R.c.P.).

(Nemertopsis Flavida (McIntosh) Beaumont (= Tetrastemma flavidum, MeIntosh, Riches, Joubin, nec Bürger).

Nemertopsis tenuIs, Bürger (= Tetrastemma flavidum, var. longissimum Joubin).

Beaumont: Fauna and Flora Valencia Harbour, Proc. Roy. Irish Acad., 1900,pp. 817 and 818, where reasons are given for relegating Tetrastemma flavidum to genus Nemertopsis Bürger.

Bürger, Naples Monograph.

The doubtful status of Nemertopsis tenuis as a species distinct from $N$. flavida, and the fact that they have rarely been distinguished with certainty make it expedient to consider them together (W.I.B.).

Very common in Plymouth Sound, between tide-marks and from dredgings, especially Duke Rk. and Millbay Ch. (T.H.R.).

Breakwater (between tide-marks), Asia Sh., Duke Rk., Millbay Ch., R. Yealm (dredging-ground); both forms occur in R. Yealm and Millbay Ch. (w.I.B.).

\section{Amphiporidæ.}

Amphiporus pulcher (Johnston): MeIntosh, Mon. Brit. Annel. i. Nemertines, Ray Soc., 1874. Beaumont, Proceed. Roy. Irish Acad., v, 1900 , p. 819. This is not the A. pulcher of Bürger's Monograph. Taken on one or two occasions on Eddystone Gds. (w.I.B.).

AMPHIPORUS LACTIFLOREUS (Johnston).

Common between tide-marks, under stones (T.H.R.): Drake's I. (E.J.A., T.V.H., R.A.T.): Rum B. (W.I.B.): Mt. Edgeumbe (E.J.A.); Wembury B. (T.V.H.).

Breeding in early spring (T.H.R.), March (w.G.).

Amphiporus dissimulans, Riches, Journ. Mar. Biol. Assoc., N.S., iii, p. 10. In considerable abundance, Millbay Ch. (T.H.R.).

Once between tide-marks, Drake's I.; dredged, Asia Sh., Millbay Ch. (common); R. Yealm (w.I.B.).

Breeding in spring and in Oct. (т.H.R.). 
[ Nemertini-contd. ]

Amphiporus bioculatus, MeIntosh.

One specimen dredged in Millbay Ch., 18.11.92, (т.H.R.).

Drepanophorus SPECTABILIS (Quatrefages).

Among weeds dredged in Cawsand B. (one specimen); dredged off Stoke Pt. (four) (T.H.R.).

Taken several times on Mewstone Ledge; Queen's Gd. (once); Eddystone Gds. (w.I.B.).

Queen's Gd.. Millbay Ch., Cawsand B. (R.A.T.): 5 m. S. of Mewstone (A.J.S.).

\section{Tetrastemmidæ.}

Tetrastemma ambiguum, Riches: Journ. Mar. Biol. Assoc., N.S., iii, p. 19.

Found Nov., 1902 (T.H.R.).

(Tetrastemma candidum (O. F. Müller).

T. VERMICULATUM (Quatrefages).

T. MELANOCEPHALUM (Johnston).

Until the British Tetrastemmidce have been thoroughly revised, any attempt to deal with the distribution of the so-called species, T. candidum, T. vermiculatum, and T. melanocephalum, is of doubtful value. Nemertines recorded under each of these names have been commonly dredged in Plymouth Sound, and occasionally found between tide-marks and among Zostera in Cawsand B. T. candidum-so-called-has also been found on the Mewstone ledge, on the inner trawling grounds, where it is abundant among Cellaria, and on the Eddystone Gds. But it may hereafter be found that two distinct species (perhaps more) have been confused as T. candidum (W.I.B.).

Tetrastemara cephalophorum, Bürger.

Millbay Ch., Duke Rk. (as Prosorhochmus Claparedii by mistake) (т.H.R.): dredged Millbay Ch., Queen's Gd., Duke Rk., R. Yealm (W.I.B.).

Oerstedia dorsalis (Zool. Dan.).

Very common in dredgings, especially in early summer among Zostera in Cawsand B. (T.H.R.): Queen's Gd., Millbay Ch. (R.A.T.): Asia Sh. (w.I.B., R.A.T.): Cawsand B. (R.A.T.): 5 m. S. of Penlee Pt. (R.A.T.): Eddystone Gds. (W.I.B., E.J.A.): found almost everywhere from shore down to $30 \mathrm{fms}$. or more-a yellow form (Bürger's var. cincta) abundant among Cellaria (w.I.B.).

Breeding in autumn (т.H.R.): Sept., Oct., Nov. (w.G.).

Oerstedia nigra (Riches): Journ. Mar. Biol. Assoc., N.S., iii, p. 14.

On Codium and other weeds from Laminarian zone (т.H.R.): Rum B. and Batten among corallines (w.I.B.).

Oerstedia immutabilis (Riches): Journ. Mar. Biol. Assoc. N.S., iii, p. 14.

Coralline pools in Wembury B., many specimens; among weeds on shore and dredged at Duke Rk. (T.H.R.).

\section{Malacobdellidæ.}

Malacobdella grossa (O. F. Müller).

In branchial cavity of Cyprina islandica, never more than one in a molluse (T.H.R., S.P.).

Ripe females in autumn (T.H.R.). 
[Nemertini-contd. ]

EUPOLIA CURTA, Hubrecht.

\section{Eupoliidæ.}

Off Borough I.; off Prawle Pt.; Eddystone Gds. (T.H.R., E.J.A.) : Millbay Ch., 2 specimens, October, 1900 (A.J.s.): Queen's Gd., one small (R.A.T.) : Mewstone Ledge; about $4 \mathrm{~m}$. S. of Mewstone; $2 \mathrm{~m}$. W. of Eddystone (W.I.B.).

Oxypolia beaumontiana, Punnett: Quart. Journ. Micr. Sci., vol. xliv, p. 555 .

Dredged off Mewstone 10.6.97 and 23.11.99, one specimen on each occasion (W.I.B., R.C.P.).

\section{Lineidæ.}

Micrella rufa, Punnett: Quart. Journ. Micr. Sci., vol. xliv, p. 548.

Yealm shore, low water, one (R.c.P.).

Lineus LoNgissimus, Gunn (=L. marinus of McIntosh).

Dredged in Yealm and outside Breakwater, occasionally on shore (T.H.R.): occasional specimens dredged and found on the shore from all parts of the Sound; Wembury B.; Yealm; Mewstone Ledge (W.I.B., R.A.T.): Eddystone Gds. (E.J.A.).

Lineus gesserensis (O. F. Müller) $=L$. obscurus, Desor.

Common everywhere in Sound between tide-marks (т.H.R., R.A.T.): Yealm (т.V.H.): Wembury B. (R.A.т.): common everywhere under stones at about mid-tide level (w.I.B.).

Breeding: Jan. (w.G.): Feb. (R.A.T.): March (T.H.R.).

Lineus LACteus (Grube).

North side of Drake's I., between tide-marks; Cawsand B., not common (T.H.R.).

Lineus Bilineatus (? Renier), MeIntosh.

Common 5 to $20 \mathrm{fms}$., especially at Duke Rk. (T.H.R.): occasionally in sand and gravel between tide-marks, Rum B. (w.I.B.): Drake's I. (R.A.T.) : Yealm Sand-bank (W.I.B., R.A.T., A.J.S.): dredged on all stony grounds in Sound (w.I.B., R.A.T.), Yealm R., S.W. of Penlee (1 $\frac{1}{2}$ miles), Eddystone Gds. (w.I.B.).

Micrura fasciolata, Ehrenberg.

Common in Sound, especially Duke Rk. (T.H.R.): dredged from stony ground in all parts of Sound (w.I.B., T.V.H., R.A.T.): RameEddystone Gds., Eddystone Gds. (w.I.B.).

Breeding: Oct. to end of year (T.H.R.).

Micrura purpurea (Dalyell), J. Müller.

Common in Sound, especially Duke Rk.; also outside Breakwater (т.H.R.): in dredgings from stony ground in all parts of Sound (w.I.B., R.A.T.) : Yealm R., Mewstone Ledge, Eddystone Gds. (W.I.B.).

Micrura aurantiaca (Grube).

Between tide-marks, Wembury B., one spec., 10.6 .92 (т.H.R.): Mewstone Ledge, Mallard, Queen's Gd., Yealm (dredged) (w.I.B.): Asia Sh. (A.J.S.), one spec. only from each locality.

Micrura lactea (Hubrecht) (= M. candida, Bürger).

Stoke Pt., 10.11.92, one dredged (T.H.R.). 
[Nemertini-contd:: Archiannelida: Polychaeta]

Cerebratulus fuscus (McIntosh).

Jennycliff B., Mallard and Cobbler Shoals, few (т.H.R.): Mallard, Queen's Gd., Drake's I., Mewstone Gds. (w.I.B.): Rum B.; Millbay Ch. (R.A.T.): Eddystone Gds., with Lepralia foliacea (w.I.B.).

Cerebratulus Pantherinus, Hubrecht (=C. marginatus (pars) Joubin). One dredged off Stoke Pt. (т.H.R.).

\section{ARCHIANNELIDA.}

Dinophilus taeniatus, Harmer: Journ. Mar. Biol. Assoc., N.S.. vol. i, p. 119, Pls. ix. and $x$.

Rock-pools in Sound far above low-water, in March and April; not found in June (S.F.H.): pools high up on limestone rock below the Laboratory and in front of West Hoe Terrace (E.J.A.).

Polygordius (APOGon MeIntosh?): Fraipont, Le Genre Polygordius, Faun. Flor. Neapel, 1887, p. 87.

Clean shell gravel off Mewstone (т.V.H., E.J.A.): Eddystone Gds. (E.J.A.).

Protodrilus Leuckarti, Hatschek: Arbeit. Zool. Inst. Wien., vol. iii, 1880, p. 79, Pl. 2.

Reared from townettings taken in September (E.J.B.).

Histriobdella homari, van Beneden: Foettinger, Archiv. Biologie, vol. v, 1884 , p. 43 อ.

Frequent on the eggs of lobsters taken by Plymouth fishermen.

Breeding during the summer months (E.J.A.).

\section{POLYCH疋TA.}

\section{Syllidæ.}

Typosyluis PROLIferA, Krohn: Langerhans, Wurmfauna v. Madeira. Zeitschr. wiss. Zool,, vol. xxxii, 1879, p. 531.

Drake's I., Millbay Ch., Queen's Gd., buoys in Sound, amongst weeds: Yealm, in red sponge (E.J.A.).

Typosyluis alternosetosa, de St. Joseph: Ann. Sci. Nat., Zool., i, 1886, p. 150 .

Eddystone Gds. (W.F.R.w.).

Syllis (EHlersia) CoRnuta, Rathke: Langerhans, Wurmfauna v. Madeira, Zeitschr. wiss Zool., vol, xxxii, 1879, p. 537.

Eddystone Gds. (w.F.R.w.).

Syldis GRacilis, Grube: Langerhans, Wurmfauna v. Madeira, Zeitschr. wiss. Zool., vol. xxxii., 1879 , p. 540 .

Amongst dredgings from Millbay and Queen's Gd. (E.J.A.).

Odontosyllis ctenostoma, Claparède: Marion et Bobretzky, Annél. Golfe Marseille, 1875, p. 42.

From the shore, under stones and amongst weeds, sponges, etc., on Drake's I., Rum B., and below Laboratory (E.J.A.).

Odontosyllis fulgurans, Claparède: Langerhans, Wurmfauna v. Madeira, Zeitschr. wiss. Zool. xxxii, 1879, p. 554.

Dredgings from Queen's Gd. (E.J.A.).

NEW SERIES,-VOL. VII. NO. 2. 
[ Polychæta--contd.]

Odontosyllis GibBa, Claparède: Marion et Bobretzky, Annél. Golfe. Marseille, 1875, p. 38.

In dredgings from (Queen's Gd. and Asia Sh. (E.J.A.).

Trypanosyllis zeBRA (Grube): Langerhans, Wurmfauna v. Madeira, Zeitschr. wiss. Zool., xxxii, 1879, p. 556.

Frequent in dredgings from Millbay Ch. and Asia Sh. Yealm dredging (E.J.A.).

Amblyosyllis spectabilis (Johnston): Catal. Brit. Non-paras. Worms, p. 195. Pl. xvi. (as Gattiola).

Common in dredgings from Millbay Ch.: less numerous Queen's Gd., Mallard, Asia (E.J.A., R.A.T., T.v.H.): Eddystone Gds., occasional (E.J.A.): sometimes in large numbers in sponges from Millbay Ch. (w.G.).

Sphaerosyllis ovigera, Langerhans: Wurmfauna v. Madeira, Zeitschr. wiss. Zool., xxxii, 1879 , p. 567.

One spec. from Queen's Gd. dredging (E.J.A.).

Autolytus PICtus (Ehlers): Borstenwürmer, 1863, p. 256.

Fairly common among stones from Millbay Ch. (w.G.): very plentiful in dredgings from Asia Sh., especially amongst sponges and Alcyonidium: occasionally dredged Millbay Ch. and Queen's Gd. (E.J.A.).

Autolytus rubropunctatus (Grube): Marion \& Bobretzky, Ann. Golfe Marseille, 1875 , p. 44 (as P. ornata): Langerhans, Wurmf. Madeira, p. 579.

Frequent in dredgings from Queen's Gd. (E.J.A.).

Myrianida pinnigera (Montagu): Trans. Linn. Soc., ix, p. 111 (M. fasciata, Aud. et Edw.).

Frequently met with in Plymouth Sound (w.G.): dredgings from Millbay Ch., Asia and Queen's Gd. (T.V.H., R.A.T., E.J.A.): amongst Ascidians and sponges from piles at Millbay Dock (R.A.T.).

\section{Hesionidæ.}

Kefersteinia cirrata (Keferstein): Claparède, Beobachtungen, 1863, p. 55, Taf. xiv.

On shore at low-water mark under stones, Drake's I., Mt. Edgeumbe, Rum B.: amongst dredgings from Millbay Ch. and Asia Sh. (E.J.A.).

Castalia punctata (Müller): Johnston, Brit. Mus. Cat. Worms, 1865,

p. 182: Grube, Jahresber. Schles. Gessellsch, 1879 (1880), p. 19.

Common in dredgings from Millbay Ch. and Asia Sb.; occasionally from Queen's Gd. (E.J.A.).

Magalia perarmata, Marion et Bobretzky: Annél. Golfe Marseille, 1875 , p. 54, Pl. 7.

Not uncommon in dredgings from Queen's Gd., Asia Sh., and Millbay Ch. (E.J.A.),

\section{Aphroditidæ.}

Aphrodita aculeata, Linn.: MeIntosh, Mon. Brit. Annel., ii, Ray Soc., 1900, p. 247.

On most fine-sand grounds off Plymouth, between 20 and 30 fms. (T.V.H., R.A.T., E.J.A.). 
[ Polychæta-contd.]

Hermione hystrix (Savigny): McIntosh, Mon. Brit. Annel. ii, Ray Soc., 1900 , p. 264.

Most frequently on gravel grounds in the neighbourhood of the Eddystone (T.v.H.) : occasionally on similar ground in about $20 \mathrm{fms}$. (R.A.T., E.J.A.).

Lepidonotus squamatus (Linn.): McIntosh, Mon. Brit. Annel. ii, Ray Soc., 1900 , p. 274.

Under stones and amongst weeds, Hydroids, Polyzoa, etc.: from low-tide mark to $30 \mathrm{fms}$. and over, common and widely distributed (T.v.H., E.J.A.).

Lepidonotus clava (Montagu): MeIntosh, Mon. Brit. Annel. ii, Ray Soc., 1900, p. 280.

Everywhere on the shore under stones, especially at extreme low water (т.V.H.): less frequently in dredgings from the Sound (E.J.A.).

Gattyana cirrosa (Pallas): McIntosh, Mon. Brit. Annel. ii, Ray Soc., 1900 , p. 285.

In dredgings from the neighbourhood of the Eddystone (т.v.H.): Yealm Sandbank and east shore, commensal in tubes of Amphitrite Johnstoni (E.J.A.).

Eunoa nodosa (M. Sars): McIntosh, Mon. Brit. Annel. ii, Ray Soc., 1900 , p. 292.

Stony ground off Prawle Point, 30 fms. (W.F.R.w.).

Lagisca floccosa (Savigny): MeIntosh, Mon. Brit. Annel. ii, Ray Soc., 1900 , p. 298.

Between tide-marks and in dredgings throughout the whole area to $30 \mathrm{fms}$. (т.V.H.).

Lagisca extenuata (Grube): McIntosh, Mon. Brit. Annel. ii, Ray Soc., 1900 , p. 307.

Not uncommon : most frequently under laminarian roots at the Breakwater, more rarely on Eddystone Gds. (т.V.H.).

Lagisca Rarispina (Sars): Malmgren, Nordiska Hafs-Annulater, 1865, p. 65 .

Eddystone Gds. (w.F.R.w., т.v.H.): Mewstone Gds. (т.v.н.).

Harmothö̈ imbricata (Linn.): McIntosh, Mon. Brit. Amnel. ii., Ray Soc., 1900 , p. 314.

Between tide-marks amongst Laminaria roots on the Breakwater: amongst Hydroids, Polyzoa, ete. on Eddystone Gds. (т.V.H.).

Harmothö̈ spinifera (Ehlers): McIntosh, Mon. Brit. Annel. ii., Ray 1900 , p. 327.

Amongst dredgings from Millbay, Queen's Gd., Asia Sh., etc., and Yealm R. : common (T.v.H., R.A.T., E.J.A.).

Harmothö̈ lunulata (Delle Chiaje): McIntosh, Mon. Brit. Annel. ii., Ray Soc., 1900 , p. 342.

On the Breakwater, among Laminaria roots and under stones at low-water mark (т.V.H.).

Harmothö̈ setosissima (Savigny): McIntosh, Mon. Brit. Annel. ii., Ray Soc., 1900 , p. 345 .

Among Polyzoa (Cellaria) and Chatopterus tubes from Eddystone Gds. (T.V.H.). 
[Polychæta-contd. .

Harmothö̈ areolata (Grube): McIntosh, Mon. Brit. Annel. ii., Ray Soc., 1900 , p. 349.

Amongst Polyzoa and Chatopterus tubes on Eddystone Gds. : not uncommon (T.V.H.).

Evarne Impar (Johnston): McIntosh, Mon. Brit. Annel, ii., Ray Soc., 1900 , p. 353.

Common between tide-marks and amongst dredgings throughout the Plymouth area (T.V.H., E.J.A.) : Eddystone Gds. (т.V.H.).

Scalisetosus communis (Delle Chiaje) : McIntosh, Mon. Brit. Annel. ii., Ray Soc., 1900, p. 372.

On the shore at Mt. Edgcumbe: amongst dredgings Millbay Ch. and Asia Sh. (R.A.T., E.J.A.).

Scalisetosus assimilis (McIntosh): McIntosh, Mon. Brit. Annel. ii., Ray Soc., 1900 , p. 377.

Amongst spines of Echinus esculentus from Mewstone and Eddystone Gds. (T.V.H., R.A.T.).

Malmgrenia castanea, MeIntosh: Mon. Brit. Annel. ii., Ray Soc., 1900 , p. 379.

Commensal on the surface of Spatangus purpureus, near the mouth of the Echinoderm : not uncommon (T.V.H.).

Halosydna gelatinosa (M. Sars): McIntosh, Mon. Brit. Annel, ii., Ray Soc., 1900 , p. 384.

On shore under stones; occasionally in deep water (т.v.н.): Wembury B., Drake's I., rocks below Laboratory (R.A.T.) : Millbay Ch., Asia Sh., Queen's Gd. (R.A.T.): Eddystone Gds. (T.v.H., E.J.A.).

Polynoe scolopendrina, Savigny: McIntosh, Mon. Brit. Annel. ii., Ray Soc., 1900 , p. 389.

Millbay Ch. and Eddystone Gds. (т.V.H.): Millbay. Ch. and Asia Sh., Mt. Edgeumbe, common in tubes of Polymnia nebulosa (R.A.T., E.J.A.). Breeding: March (R.A.T.).

Acholö̈ Astericola (Delle Chiaje): McIntosh, Mon. Brit. Annel. ii., Ray Soc., 1900 , p. 397.

In the ambulacral groove of Astropecten irregularis: common (T.V.H., R.A.T., E.J.A.)

Sthenelais boa (Johnston): McIntosh, Mon. Brit. Annel., ii., Ray Soc., 1900 , p. 408.

Common in sand between tide-marks, Rum B., Drake's I., Mt. Edgcumbe, Wembury B., Yealm R. (T.V.H., R.A.T., E.J.A.) : occasionally amongst dredgings from Millbay and Asia Sh. (T.V.H., E.J.A.) : Mewstone Gds. (T.V.H.).

Pholö minuta (Fabricius): MeIntosh, Mon. Brit. Annel. ii., Ray Soc., 1900 , p. 437.

Common amongst dredgings from Millbay Ch., less frequent from Asia Sh. and Queen's Gd. (E.J.A.).

\section{Amphinomidæ.}

Euphrosyne foliosa, Aud. et Edw.: McIntosh. Mon. Brit. Annel., part ii., Polychaeta, Ray Soc., 1900, p. 234. Pl. xxiv.

Occasional specimens in dredgings from Queen's Gd., Asia Sh., Millbay Ch. (T.V.H., R.A.T., E.J.A.) : Eddystone Gds. (E.J.A.). 


\section{Phyllodocidæ.}

Eulalia viridis (Müller): Johnston, Catal. Worms. Brit. Mus., 1865, p. 178. Pl. xvi.

Common on rocky shores all round the Sound (R.A.T., E.J.A.): Eddystone Gds. (E.J.A.).

Eulalia punctifera (Grube): de St. Joseph, Ann. Sci. Nat. Zool., v., 1888, p. 289 . Pl. xii.

In dredgings from Millbay Ch., Asia Sh., and Yealm; on the shore at Mt. Edgeumbe and in Yealm estuary (E.J.A.).

Eulalia aurea, Gravier: Recherches sur les Phyllodociens., Bull. Sci. France et Belg. xxix., 1896, p. 309.

Common in dredgings from Millbay Ch., Asia Sh., and Queen's Gd., occasionally from Yealm (E.J.A.).

Breeding: March, April, May, June, July (E.J.A.).

Eulalia Claparèdei, de St. Joseph: Ann. Sci. Nat. Zool., v., 1888, p. 285. Pl. xi.

Not infrequent in dredgings from Millbay Ch., Asia Sh., and occasionally Queen's Gd.

Breeding : May, eggs brick-red (E.J.A.).

Eulalia obtecta, Ehlers: Borstenwürmer, 1868, p. 169 . Pl. vii.

Frequent in dredgings from Queen's Gd.: occasionally from

Asia Sh., Millbay Ch., and Mewstone Echinoderm Gd. (E.J.A.).

Eulalia ornata, de St. Joseph: Ann. Sci. Nat. Zool., v., 1888, p. 291.

In dredgings from Millbay Ch. and Asia Sh. (E.J.A.).

Breeding: April-July (E.J.A.).

Eulalia Pallida, Claparède: de St. Joseph, Ann. Sci. Nat. Zool., v., 1888 , p. 294.

Very numerous in dredgings from Millbay Ch., Asia Sh., and Queen's Gd. (E.J.A.).

Breeding: May to July: eggs green (E.J.A.).

Pterocirrus macroceros (Grube): de St. Joseph, Ann. Sci. Nat. Zool,, v., 1888, p. 300 . Pl. xii.

Occasional specimens Queen's Gd. (E.J.A.).

Phyllodoce laminosa, Savigny, de St. Joseph, Ann. Sci. Nat. Zool. v., 1888 , p. 274 . Pl. xi.

Between tide-marks Drake's I., Breakwater : in dredgings Millbay Ch., Asia Sh., Duke Rock (T.V.H., E.J.A.).

Phyllodoce maculata (Müller): Johnston, Catal. Worms. Brit. Mus., 1865, p. 177.

Common in dredgings from Millbay Ch. and Asia Sh. (often in great numbers) (w.G., T.v.H., E.J.A.): shore at Drake's I. (E.J.A.) : Yealm dredgings (T.V.H.).

Breeding: Jan. Feb. (w.G.) : April, May. Eggs orange-brown (E.J.A.).

Phyllodoce rubiginosa, de St. Joseph: Ann. Sci. Nat. Zool. v, 1888, p. 282.

Frequent in dredgings from Millbay Ch., Asia Sh., and Drake's I. (E.J.A.). 
[Polychæta-contd.]

Phyllodoce Paretri, Blainville: Cuvier, Règne animal, Annélides. Pl. 13. Fig. 1.

Mewstone Ledge (E.J.A.): Stoke Pt. Gds. (S.P.).

Eteone PICta, Quatrefages: Hist. Nat. Annel. ii, p. 147. Pl. 18.

Occasional specimens from dredgings, Millbay Ch., Asia Sh., Queen's Gd., Barn Pool (E.J.A.).

\section{Tomopteridæ.}

Tomopteris helgolandica, Greef: Apstein, Alciopiden und Tomopteriden der Plankton-Expedition, Kiel, 1900, p. 38. Pl. x.

In townettings Oct. (T.V.H.): Dec. Jan. June (L.H.G.).

\section{Nereidæ.}

Nereis cultrifera, Grube: Ehlers, Borstenwürmer, 1868, p. 461. Pl. xxi.

Common on the shores, especially in muddy gravel, all round the Sound, Wembury B., Yealm Estuary (R.A.T., E.J.A.).

Nereis diversicolor, O. F. Müller: Ehlers, Borstenwürmer, 1868, p. 554. Pl. xxii.

Common in the mud flats of the Tamar and Plym estuaries, seldom in the Sound; found only where the density of the water is low (E.J.A.).

Nereis Dumerilli, Aud. et Edw. : Ehler's, Borstenwürmer, 1868, p. 535. Pl. $\mathrm{xx}$.

Small specimens common in all dredgings from the Sound, Millbay, Asia Sh., Queen's Gd., and from Yealm R.; larger specimens chiefly from Queen's Gd.; occasional specimens on the shore under Laboratory and Barn Pool (E.J.A.).

Nereis fucata, Savigny : Ehlers, Borstenwürmer, 1868, p. 546. Pl. xxi.

In shells of Buccinum undatum inhabited by Eupagurus Bernhardus; Mewstone Gds., Rame to Eddystone Gds., Eddystone Gds. (E.J.A.) : Yealm R. (s.P.).

Breeding: May (w.G.).

Nereis Irrorata, Malmgren: de St. Joseph, Ann. Sci. Nat., Zool., v, 1888 , p. 263 , and $x x, 1895$, p. 215.

Not uncommon in sand between tide-marks, Drake's I., Mt. Edgeumbe, Jennycliff (rare), Wembury B., Yealm Estuary south shore. Small specimens amongst dredgings Queen's Gd. and Asia Sh. (E.J.A.).

Nereis Longissima, Johnston: de St. Joseph, Ann. Sci. Nat., Zool., v, 1898 , p. 304. Pl. xvi, and xvii.

Occasional specimens in fine sand between tide-marks, Drake's I. and Mt. Edgeumbe; more common on shores of Yealm estuary; one specimen of Heteronereis stage taken in Cattewater (E.J.A.). In mud-bank of Cattewater near Oreston (J.T.C.).

Nereis pelagica, Linnæus : Ehlers, Borstenwürmer, 1868, p. 511. Pl. xx.

Frequent amongst weeds on rocky shores and from dredgings in all parts of the Sound; also. Wembury B. and Yealm R. (T.V.H., E.J.A.). 
[ Polychæta-contd.]

\section{Nephthydidæ.}

Nephthys C.æCA (Fabricius): de St. Joseph, Ann. Sci. Nat., Zool., xvii, 1894 , p. 16.

Large specimens on sandy shores, especially Drake's I., Rum B., and Yealm Sand-bank (T.V.H., R.A.T., E.J.A.).

Nephthys Hombergi, Aud, et Edw.: de St. Joseph, Ann. Sci. Nat., Zool., xvii, 1894 , p. 3.

Shore between tide-marks, Drake's I., Jennycliff, Bovisand (R.A.T., E.J.A.): dredged on Mewstone 'Amphioxus' Gd. (R.A.T.), and Eddystone Gds. (T.V.H.).

Nephthys cirrosa, Ehlers: Borstenwürmer, 1868, p. 624. Pl. xxiii.

In sand between tide-marks, Drake's I. and Yealm estuary (E.J.A.).

\section{Eunicidæ.}

Eunice Harassi, Aud. et Edw.: Ehlers, Borstenwürmer, 1868, p. 312. Pls. xiii \& xiv.

On the shore Drake's I., Rum B., Breakwater (R.A.T., E.J.A.): Wembury B. (E.J.A.) : in dredgings from Duke Rk. (W.G., T.V.H.) : Queen's Gd., Asia Sh., Millbay Ch. (R.A.T., E.J.A.): south of Breakwater Fort (w.G.) : Rame to Eddystone Gds. (E.J.A.).

Onuphis conchilega, M. Sars: Oersted, Grönlands Annulata Dorsibranchiata, 1843, p. 20, Pl. iii. (as O. Eschrichtii): ef. de St. Joseph, Ann. Sci. Nat. Zool., v, 1888, p. 194.

On coarse shell-gravel grounds, in depths from 20 to $30 \mathrm{fms}$. off the Mewstone, off Stoke Pt., S. of Rame Head, and off Eddystone (E.J.A., R.A.T.).

Hyalinecia tubicola (Müller): Ehlers, Borstenwürmer, 1868, p. 297.

Common on muddy-gravel grounds from 20 to $30 \mathrm{fms}$ : off Mewstone, Rame to Eddystone and Eddystone Gds. (E.J.A., R.A.T.)

Marphysa sanguinea (Montagu): de St. Joseph, Ann. Sci. Nat., v, 1888 , p. 201.

Frequent on the shore in crevices of rock, especially on the bridge between Drake's I. and Mt. Edgcumbe; also Rum B., Wembury B., and Yealm Estuary (W.G., T.V.H., R.A.T., E.J.A.).

Marphysa Belli, Aud. et Edw.: Hist. Nat. Lit. France, ii, p. 149, Pl. iii.

On the shore between tide-marks N. side Drake's I., Rum B.; most frequent in Zostera beds at mouth of Yealm R. (E.J.A.).

Lysidice ninetta, Aud. et Edw.: Hist. Nat. Lit. France, ii, p. 161, Pl. iiib.

Frequent in Plymouth Sound, on the shore between tide-marks (Rum B., Drake's I.), and in dredgings (Asia Sh., Millbay Ch., Queen's Gd.); on shore Wembury B. and Reny Rks.; dredged in Yealm R. (E.J.A.).

Nematonereis unicornis (Grube): de St. Joseph, Ann. Sci. Nat., v, 1888, p. 207.

Between tide-marks N. side of Drake's I. and Mt. Edgcumbe; amongst dredgings from Queen's Gd., Asia Sh., and Millbay Ch. (E.J.A.). 
[Polychæta-contd.]

Lumbriconereis impatiens, Claparède: de St. Joseph, Ann. Sci. Nat. Zool., v, 1898, p. 279.

Coarse grounds between Eddystone and Rame; very abundant on Queen's Gd. in spring of 1903 (E.J.A.).

Lumbriconereis Latreillei, Aud. et Edw.: de St. Joseph, Ann. Sci. Nat. Zool., v, 1898, p. 276.

Shores of the Sound, between tide-marks (Drake's I., Mt. Edgcumbe, Rum B.) ; amongst dredgings from Millbay Ch., Asia Sh., Queen's Gd., Yealm R., and Eddystone Gds. (E.J.A.).

Maclovia Iricolor (Montagu): Willey, Journ. Mar. Biol. Assoc., vi, 1900 , p. $98=M$. gigantea (Quatrefages).

Occasional specimens at low-water mark on shores of Sound (E.J.A.).

Staurocephalus rubrovittatus, Grube: Ehlers, Borstenwürmer, 1868, p. 424 .

Frequent in dredgings from Millbay Ch., Queen's Gd., and Asia Sh. (E.J.A.).

Staurocephalus ciliatus, Keferstein: Ehlers, Borstenwürmer, 1868, p. 440 .

Recorded once from Queen's Gd. dredging (E.J.A.).

Staurocephalus pallidus, Langerhans: Zeits. wiss. Zool., xxxiii, 1879, p. 300.

Recorded once from Asia Sh. dredging; resembles S. ciliatus, Kef., excepting for absence of eyes (E.J.A.).

Ophryotrocha puerilis, Clpd. et Meczn. : de St. Joseph, Ann. Sci. Nat. Zool., xx, 1895, p. 210 (as Paractius mutabilis, n. sp.).

Dredgings from Duke Rk. (т.V.H.): often common in Laboratory tanks (E.J.A.).

Breeding: Aug. (w.G.). Spawned in tanks, May (A.J.S.).

\section{Glyceridæ.}

Glycera convoluta, Keferstein : de St. Joseph, Ann. Sci. Nat. Zool., xvii, 1894 , p. 27 , Pl. ii.

Between tide-marks Drake's I., Jennycliff, Yealm Estuary; in dredgings from Millbay Ch., Rame to Eddystone and Eddystone Gds. (E.J.A.).

Glycera lapidum, Quatrefages: Arwidsson, Studien über die Familien Glyceridae und Goniaididae, Bergens Mus. Aarb., 1898, p. 15, Figs. 7, 8, and 55 .

Between tide-marks Wembury B. (T.v.H., E.J.A.): dredged in shell gravel off Mewstone (T.V.H., E.J.A.) and near Queen's Gd. (E.J.A.).

Glycera gigantea, Quatrefages: de St. Joseph, Ann. Sci. Nat. Zool, xvii, 1894 , p. 22 , Pl. ii.

Between tide-marks, Drake's I. (w.G., R.A.T.) : Mewstone Amphioxus Gd. (E.J.A.).

Goniada maculata, Oersted: Ehler's, Borstenwürmer, 1868, p. 704, Pl. xxiv.

One specimen from Plymouth Breakwater (E.J.A.). 
[Polychæta-contd.]

\section{Sphærodoridæ.}

Ephesia gracilis, Rathke: de St. Joseph, Ann. Sci. Nat. Zool., xvii, 1894, p. 38, Pl. iii=Spharodorum peripatus, Johnston.

Frequent in dredgings from Millbay Ch. and Asia Sh.; occasionally also from Queen's Gd. and from between tide-marks on Drake's I. and Mt. Edgeumbe (E.J.A.).

\section{Ariciidæ.}

Aricia Cuvieri, Aud. et Edw.: de St. Joseph, Ann. Sci. Nat. Zool, xvii, 1894, p. 91.

One specimen dredged off the Mewstone (E.J.A.).

Scoloplos ARmiger (Müller): de St. Joseph, Ann. Sci. Nat. Zool., xvii, 1894 , p. 94 , Pl. v.

In dirty sand and between layers of shale at Rat I. (Hamoaze) (w.G.): in sand at Drake's I., Rum B., and the Yealm Estuary (E.J.A.).

\section{Spionidæ.}

ScolelePIS VUlGARIS, Johnston (probably the same as $S$. fuliginosa Claparède, var. macrochata major of Mesnil: Bull. Sci. France et Belg., xxix, 1896, p. 138).

Mud between tide-marks, Rum B. and Wembury B.; numerous (E.J.A.).

Scolelepis Giardi (de Quatrefages): Mesnil, Bull Sci. France et Belg., xxix, 1896, p. 140.

One specimen from mud in Rum B. (E.J.A.).

Nerine cirratulus (Delle Chiaje): Mesnil, Bull. Sci. France et Belg., xxix, 1896 , p. 152.

Fine gravel between tide-marks on eastern side of Plymouth Sound (E.J.A.).

Nerine coniocephala, Johnston ( $=N$. foliosa, Aud. et Edw.: Mesnil, Bull. Sci. France et Belg., xxix, 1896, p. 165).

In sand between tide-marks, E. side of Drake's I. (E.J.A.) : Wembury B. (т.v.H.).

Aonides oxycephala (Sars): Mesnil, Bull. Sci. France et Belg., xxix, 1896, p. 242 , Pl. x.

In Zostera bed at low-water mark, eastern shore of Yealm mouth; very numerous (E.J.A.).

Polydora ciliata (Johnston): Mesnil, Bull. Sci. France et Belg., xxix, 1896, p. 210, Pl. xiv.

Boring in limestone of the Breakwater and in limestone dredged from Millbay Ch. (E.J.A.).

Polydora flava, Claparède: Mesnil, Bull. Sci. France et Belg., xxix, 1896 , p. 182 , Pl. xi.

At Rum B. and Rat I. (Hamoaze); common in crevices of shale (w.G.): Rum B. in crevices of shale (E.J.A.) : dredgings from Yealm R. (E.J.A.).

Breeding: Feb. (w.G.). 
[ Polychæta-contd.]

Polydora C.ecA, Oersted: Mesnil, Bull. Sci. France et Belg., xxix, 1896, p. 191, Pl. xii.

Eddystone Gds. (т.V.H.).

Polydora hoplura, Claparède: Annél. Chétop. Golfe Naples, 1868, p. 318, Pl. xxii.

Boring in the limestone of the Plymouth Breakwater (E.J.A.).

\section{Disomidæ.}

Pecilochetus serpens, Allen: Quart. Journ. Micr. Sci., vol. xlviii, 1904, p. 79 , Pls. $7-12$.

In sand at low tide, S. of Batten Castle; larvæ not uncommon in the plankton during the summer months (E.J.A.).

\section{Chætopteridæ.}

Chatopterus variopedatus, Renier: Joyeux-Laffice, Arch. Zool. Exp. et Gen., viii, 1890 , p. 244 , Pls. $15-20$.

Common in muddy gravel on Eddystone Gds. and between Rame and Eddystone (E.J.A.): occasional specimens Duke Rk. (T.V.H.), Asia Sh. (R.A.T.), Millbay Ch. (R.A.T., E.J.A.), and Mewstone Echinoderm Gd. (R.A.T.): Stoke Pt. Gds. (s.P.).

Larvæ in townettings July to October (w.G.).

\section{Magelonidæ.}

Magelona papillicornis, Fr. Müller: de St. Joseph, Ann. Sci. Nat. Zool., xvii, 1894, p. 83.

In fine sand near low-water mark at Jennycliff B. (R.A.T., E.J.A.), under Batten Castle, and on S. shore of Yealm Estuary (E.J.A.).

Larvæ in townets in July and August (E.J.A.): in September (E.J.B.).

\section{Ammocharidæ.}

Owenia fusiformis (Delle Chiaje): de St. Joseph, Ann. Sci. Nat. Zool., v, 1898, p. 397, Pl. xxii.

In fine sand near low-water mark at Jennycliff B. (R.A.T., E.J.A.) and under Batten Castle (E.J.A.).

\section{Cirratulidæ.}

Audouinia tentaculata (Montagu) : de St. Joseph, Ann. Sci. Nat. Zool., xvii, 1894, p. 49, Pl. iii.

Common in gravel and sand just below high-water mark on all shores, both inside and outside the Sound (т.v.H., R.A.T., E.J.A.): occasional small specimens from dredgings at Millbay Ch. (E.J.A.).

Dodecaceria concharum, Oersted: de St. Joseph, Ann. Sci. Nat. Zool., v, 1898, p. 346, Pl. xx.

Boring in limestone on Plymouth Breakwater, abundant; Millbay Ch. (E.J.A.).

\section{Terebellidæ.}

Amphitrite Gracilis, Grube: de St. Joseph, Ann. Sci. Nat. Zool., xvii, 1894, p. 198.

Common in sand between tide-marks and between layers of shale in Rum B. and at Mt. Edgeumbe, Yealm Sand-bank, and Wembury B.; dredged Millbay Ch. and Eddystone Gds. (E.J.A.). 
Polychæta_contd.]

Amphitrite Johnstoni, Malmgren : de St. Joseph, Ann. Sci. Nat. Zool., v, 1898 , p. 421 .

In sand and gravel near low-water mark along the S. shore of the Yealm R.; most common on east shore where the stream divides (R.A.T., E.J.A.).

Terebella lapidaria (Kähler), Linn.: de St. Joseph, Ann. Sci. Nat. Zool., xvii, 1894, p. 202, Pls. viii. and ix.

Common in crevices of shale at Rum B. (R.A.T., E.J.A.).

Polymnia nebulosa (Montagu): de St. Joseph, Ann. Sci. Nat. Zool., xvii, 1894 , p. 219 , Pl. ix.

Very common between tide-marks at Mt. Edgeumbe (R.A.T., E.J.A.): occasional specimens on the shore at Rum B. and in dredgings from Millbay Ch., Asia Sh., Queen's Gd., and Yealm R. (R.A.T., E.J.A.): Eddystone Gds. (T.V.H.).

Polymnia nesidensis (Delle Chiaje) : de St. Joseph, Ann. Sci. Nat. Zool., xvii, 1894 , p. 225 , Pl. x.

Between tide-marks, Rum B., Mt. Edgcumbe, Wembury B.; dredged at Asia Sh., Yealm R., and Cawsand B. (E.J.A.).

Lanice conchilega (Pallas): de St. Joseph, Ann. Sci. Nat. Zool., xvii, 1894 , p. 211.

Common on sandy shores inside and outside the Sound; occasionally dredged Queen's Gd.; Eddystone Gds. (T.v.H., R.A.T., E.J.A.).

Nicolea zostericola (Oersted): Malmgren, Nordiska Hafs-Annulater, Öfer. K. Vet. Akad. Förh., 1865, p. 381, Pl. 26.

Common between tide-marks on Reny Rks., less frequent Drake's I., N. side; also from dredgings in Yealm R. (E.J.A.).

Thelepus setosus (Quatrefages): de St. Joseph, Ann. Sci. Nat. Zool., xvii, 1894 , p. 230 , Pl. x.

Dredged at Millbay Ch., Queen's Gd, Eddystone Gds. (E.J.A.).

Polycirrus aurantiacus, Grube: de St. Joseph, Ann. Sci. Nat. Zool., xvii, 1894 , p. 239.

Common in dredgings from Millbay Ch. and Yealm R; less frequent in those from Asia Sh., Queen's Gd., and Duke Rk. (T.v.H., R.A.T., E.J.A.) : Eddystone Gds. (E.J.A.).

Breeding: July (w.G.); April (E.J.A.).

Polycirrus caliendrum, Claparède: de St. Joseph, Anu. Sci. Nat. Zool., xvii, 1894, p. 237.

In dredgings from Millbay Ch., Queen's Gd., and Asia Sh. (E.J.A.).

Loima medusa (Savigny): Malmgren, Nord. Hafs-Ann., p. 380, Pl. xxv; Annul. Polych., p. 217, Pl. xiv.

Amongst shell gravel near low-water mark on N. side of Drake's I.; tubes from Queen's Gd.; Yealm R. shore (E.J.A.).

Terebellides Stroemi, Sars: Malmgren, Nord. Hafs-Annul., 1865, p. 396, Pl. xix, Fig. 48.

One specimen dredged four miles S.W. by S. of Rame Head (E.J.A.). 
[Polychæta-contd.]

\section{Ampharetidæ.}

Melinna adriatica, v. Marenzeller: Adriatische Anneliden. Sitzb. Akad. Wien, lxix, 1874, p. 472, Pl. vii.

Common in soft mud in Plymouth Sound, on the shore and in deeper water (E.J.A.).

Amphicteis Curvipalea, Claparède: de St. Joseph, Ann. Sci. Nat. Zool., xvii, 1894, p. 163, Pl. vii.

One specimen dredged four miles S.W. by S. of Rame Head (E.J.A.).

\section{Amphictenidæ.}

Pectinaria (Lagis) Koreni, Malmgren: de St. Joseph, Ann. Sci. Nat. Zool., v, 1898, p. 405, Pls. xxii and xxiii.

Common in sand near low-water mark S. of Batten Castle (E.J.A.).

Pectinaria (Amphictene) auricoma (Müller): Malmgren, Nord. HafsAnnulat., 1865, p. 357, Pl. 18.

Eddystone Gds. (т.V.H.).

\section{Capitellidæ.}

Capitella capitata (Fabricius): Eisig, Die Capitelliden des Golfes von Neapel, 1887, p. 849.

Common in black mud from between tide-marks, Wembury B. and Rum B. (E.J.A.).

Notomastus rubicundus, Keferstein: Eisig, Die Capitelliden des Golfes von Neapel, 1887, p. 863.

South shore of Yealm Estuary (near the mouth), common (E.J.A.).

Notomastus latericeus, Sars: Sars, Koren, Danielsen, Fauna littoralis Norvegiae, ii, 1856, p. 9, Pl. ii.

Shore near low-water mark, Yealm Estuary; not frequent (E.J.A.).

\section{Opheliidæ.}

Ammotrypane aulogaster, Rathke: Beiträge zur Fauna Norwegens, 1840, p. 188 , Pl. x.

Dredged off Duke Rk. (w.G.): Mewstone Amphioons Gd. (E.J.A.). Polyophthalmus PICtus (Dujardin): de St. Joseph, Ann. Sci. Nat. Zool., v, 1898, p. 385 .

Between tide-marks Wembury Bay (A.J.s.): Reny Rks. (E.J.A.).

\section{Arenicolidæ.}

Arenicola marina, Linn.: Gamble, Anatomy and Classification of Arenicolidae: Quart. Journ. Micr. Sci., xliii, p. 419.

Fine sand between tide-marks Rum B., Drake's I., Mt. Edgcumbe, Wembury B., Yealm R. (T.V.H., R.A.T., E.J.A.).

Postlarval stages of Arenicola in townettings February (w.G., w.B.B.).

Arenicola Grubei, Claparède: Gamble, Anatomy and Classification of Arenicolidae, Quart. Journ. Micr. Sci, xliii, p. 419.

With $A$. ecaudata near the bases of rocks in a deposit composed of sand and small stones (F.W.G.): Rum B., Wembury B., Drake's I. (E.J.A.). 
[Polychæta-contd.]

Arenicola ecaudata, Johnston: Gamble, Anatomy and Classification of Arenicolidae, Quart. Journ. Micr. Sci., xliii, p. 419.

With $A$. Grubii near the bases of rocks in a deposit composed of sand and small stones (F.W.G.): Rum B., rocks below Laboratory (R.A.T.) : Wembury B. (R.A.T., E.J.A.) : Drake's I. (т.v.H.).

\section{Scalibregmidæ.}

Scalibregma Inflatum, Rathke: Ashworth, Quart. Journ. Micr. Sci, xlv, 1902 , p. 237.

In muddy gravel at low-water mark on southern shore of Yealm $\mathrm{R}$., just below the junction of the two rivers; two specimens, $10 / 9 / 00$ (E.J.A.).

Sclerocheilus minutus, Grube: de St. Joseph, Ann. Sci. Nat. Zool., xvii, 1894, p. 104, Pl. v.

Frequent in dredgings from Millbay $\mathrm{Ch}$; occasionally from Asia Sh. and off the Mewstone (E.J.A.).

Chlorhæmidæ.

Stylarioides (Trophonia) plumosa (Müller): de St. Joseph, Ann. Sci. Nat. Zool., xvii, 1894, p. 101.

Dredgings from Millbay Ch. and Asia Sh.; also $2 \frac{1}{2}$ miles off Stoke Pt. (E.J.A.).

Siphonostoma (Flabelligera) affinis, M. Sars: Newbigin, Ann. Mag. Nat. Hist., ser. 7, vol. v, 1900 , p. 190, Pl. iv.

Between tide-marks at Drake's I. (R.A.T., T.V.H., E.J.A.) : Renny Rks. (R.A.T., E.J.A.) : under Rame Head (т.V.H.) : Wembury B. (E.J.A., A.J.S.): in dredgings from Millbay Ch., Queen's Gd. (R.A.T., T.v.H.) : Mewstone Gds. (E.J.A., R.A.T.): on Echinus acutus (R.A.T.).

\section{Sabellidæ.}

Sabella pavonina (Savigny): de St. Joseph, Ann. Sci. Nat. Zool., xvii, 1894 , p. 267 , Pls. $x$. and xi.

Common on the shore in Yealm R. and also found in Yealm dredgings (R.A.T., E.J.A.): Eddystone Gds. (E.J.A.).

Branchiomma vesiculosum (Montagu): de St. Joseph, Ann. Sci. Nat. Zool., xvii, 1894 , p. 300, Pl. xi.

Occasional specimens Yealm Sand-bank, Rum B. (R.A.T.) : Drake's I., Yealm south shore near mouth, Barn Pool, and shore S. of Batten Castle (E.J.A.).

Dasychone вombyx (Dalyell): de St. Joseph, Ann. Sci. Nat. Zool, xvii, 1894 , p. 309 , Pls. xi and xii.

In dredgings from Duke Rk., Queen's Gd., Asia Sh., and Millbay Ch., occasionally (E.J.A.): Eddystone Gds. (т.v.H.).

Potamilla reniformis (Muiller): de St. Joseph, Ann. Sci. Nat. Zool., xvii, 1894, p. 292, Pl. xi.

Large specimens from shore in Jennycliff B.; found also in Wembury B. (shore); not uncommon boring in limestone of Breakwater (E.J.A.).

Potamilla Torelli, Malmgren: de St. Joseph, Ann. Sci. Nat. Zool., xvii, 1894, p. 296.

Common in dredgings from Millbay Ch. and from Yealm R. (E.J.A.). 
[ Polychrta-contd.]

Potamilia incerta, Langerhans: de St. Joseph, Ann. Sci. Nat. Zool., xvii, 1894, p. 299.

Recorded once from Yealm dredging (E.J.A.).

Bispira volutacornis (Montagu): de St. Joseph, Ann. Sci. Nat. Zool., xvii, 1894, p. 286.

In cracks between rocks at extreme low-tide mark on Renny Rks.; Jennycliff B. shore (R.A.T.).

Myxicola infundibulum (Renier): de St. Joseph, Ann. Sci. Nat. Zool., v, 1898 , p. 433 , Pl. xxiii.

North side Drake's I. (R.A.T.): Barn Pool (E.J.A.): Rams Cliff Point (s.P.).

\section{Serpulidæ.}

Serpula vermicularis, Linnæus: de St. Joseph, Ann. Sci. Nat. Zool,, xvii, 1894 , p. 328.

Occasionally in the Sound: Mewstone Gds. (R.A.T.): Rame to Eddystone Gds., and Eddystone Gds. (E.J.A.).

Pomatoceros triqueter (Linn.): de St. Joseph, Ann. Sci. Nat. Zool., xvii, 1894, p. 353, Pl. xiii.

Common attached to shells and stones on all grounds from the shore to $30 \mathrm{fms}$. (E.J.A.).

Hydroides norvegica (Gunn.): de St. Joseph, Ann. Sci. Nat. Zool., v, 1898, p. 440.

Common on stones and shells, 0-30 fms. (E.J.A.).

Filograna implexa (Berkeley): de St. Joseph, Ann. Sci. Nat. Zool., xvii, 1894 , p. 335 , Pls. xii and xiii.

In quantity Millbay Ch., piles of Promenade Pier (E.J.A.): on piles at entrance to Millbay Docks (R.A.T.): Breakwater (т.V.H.): occasionally met with in all dredgings from Sound and outside to Eddystone Gds. (E.J.A.).

SpIrorbis borealis, Daudin : de St. Joseph, Ann. Sci. Nat. Zool., xvii, 1894 , p. 345 , Pl. xiii.

Common on weeds, especially Fucus, and on stones on all shores. (E J.A.).

Protula tubularia (Montagu): de St. Joseph, Ann. Sci. Nat. Zool, xvii, 1894 , p. 362 , Pl. xiii.

Mewstone Gds., Rame-Eddystone Gds., Eddystone Gds. (R.A.T., E.J.A.).

\section{Hermellidæ.}

Sabellaria alveolata (Linn.): de St. Joseph, Ann. Sci. Nat. Zool., xvii, 1894, p. 160.

Common attached to rocks on sandy shores at Whitsand B. (E.J.A.).

Sabellaria spinulosa, Leuckart: de St. Joseph, Ann. Sci. Nat. Zool., xvii, 1894 , p. 154 .

Attached to shells, etc., in dredgings from Queen's Gd., Duke Rk., Asia Sh., Millbay Ch., and Eddystone Gds. (E.J.A.).

Breeding: May (w.G.); September (E.J.A.).

Pallasia murata, Allen: see p. 299.

One specimen from gravel off Stoke Pt.; tubes frequent on Mewstone shell-gravel Grounds (E.J.A.). 
[Myzostomaria: Oligochæta: Gephyrea: Hirudinea: Chætognatha: Branchiopoda]

\section{MYZOSTOMARIA.}

Myzostomum cirriferum, Leuckart: Benham, Cambridge Nat. Hist., Worms, etc., fig. 186.

Common on Antedon bifida from the Sound (E.J.A.).

\section{OLIGOCHÆTA.}

Clitellio arenarius, Claparède: Beddard, Journ. Mar. Biol. Assoc., N.S., i, p. 69.

Mud at Drake's I. and on the shores of the Sound (F.E.B.).

Clitellio Ater, Claparède: Beddard, Journ. Mar. Biol. Assoc., N.S., i, p. 70.

Mud on Drake's I. and on shores of Sound (F.E.B.).

Pachydrilus sp.: Beddard, Journ. Mar. Biol. Assoc., N.S., i, p. 69.

Common in gravel between tide-marks, Rum B. (F.E.B.).

\section{GEPHYREA.}

Nomenclature :-Selenka. E., Die Sipunculiden., Reisen. Archipel. Philipp. Semper., Bd. iv, 1883-1885.

Petalostoma minutum, Keferstein.

Rum B., common in crevices of shale (T.V.H., R.A.T., E.J.A.).

Phascolion strombi, Montagu.

Frequently met with in shells of Aporrhais pes-pelecani; Mewstone Gds. (T.V.H., E.J.A.).

Thalassema neptuni, Gaertner.

Common in holes in rock, Rum B., Wembury B., Drake's I.; one spec. Yealın Sand-bank; in stones dredged from Millbay Ch., Asia Sh., and (rarely) Queen's Gd. (R.A.T.).

Phoronis hippocrepia, Wright: Shipley, Cambridge Nat. Hist., 1896.

Abundant in the Sound (w.G.): Duke Rk. (т.v.H.): Millbay Ch. (T.v.H., E.J.A.): Asia Sh.; Queen's Gd. (s.P.).

\section{HIRUDINEA.}

Pontobdella muricata, Linnæus: W. C. McIntosh, Mar. Invert. \& Fishes St. Andrews, p. 114, fig.

Occasionally on the outside grounds; parasitic on the skate (s.P.).

Breeding: July (E.W.L.H.).

\section{CHÆTOGNATHA.}

SAGITTA BIPUnctata (Quoy \& Gaimard): Grassi, I Chetognati, Fauna u. Flora Golf. Neapel., v.

In townettings throughout the year (L.H.G.).

\section{BRANCHIOPODA.}

Evadne Nordmanni, Lovén: C. Apstein, Nordisches Plankt., pt. vii, Cladoc., p. 12, fig.

1892, appeared beginning of July and still present in Oct. (E.J.B.): 1895, constantly present in Aug., disappeared early Oct. (T.V.H.): Apr.-May, 1899 (P.T.c.): May, July, Sept., 1903; Mar.-June, 1904 (L.H.G.).

Carrying ova: Aug. (т.v.H.). Winter eggs produced from about mid-Sept. (E.J.B.). 
[Branchiopoda-contd. : 0stracoda: Copepoda]

Podon intermedius, Lilljeborg: C. Apstein, Nordisches Plankt., pt. vii, Cladoc., p. 15, fig.

1892, became less abundant in July and disappeared about mid-Sept. (E.J.B.): 1895, constantly present in Aug., disappeared early in Oct. (т.v.H.): Plymouth neighbourhond, Apr.-Sept., 1903 ; Apr.-July, 1904 (L.H.G.).

Carrying ova: Aug. (т.V.H.)

Podon Leuckarti, G. O. Sars: C. Apstein, Nordisches Plankt., pt. vii, Cladoc., p. 13, fig.

Plymouth neighbourhood, Apr. 1904, rare (L.H.G.).

\section{OSTRACODA.}

\section{Cypridinidæ.}

Philomedes interpuncta, Baird: G. S. Brady \& Norman, Trans. Dublin Soc., ser. 2, vol. v, p. 656, fig.

Plymouth (A.M.N.).

\section{Asteropidæ.}

Asterope Marie (Baird): G. S. Brady \& Norman, Trans. Dublin Soc., ser. 2 , vol. v, p. 630 , fig.

Plymouth (A.M.N.).

\section{Cytheridæ.}

Cythere oblonga, Brady: G. S. Brady, Trans. Linn. Soc., vol. xxvi, p. 400 , fig.

Plymouth (A.M.N.).

Cythere quadridentata, Baird: G. S. Brady, Trans. Linn. Soc., vol. xxvi, p. 413 , fig.

Plymouth (A.M.N.).

Cythere emaciata, Brady: G. S. Brady, Trans. Linn. Soc., vol. xxvi, p. 414, fig.

Plymouth (A.M.N.).

Cythere runcinata, Baird: G. S. Brady \& Norman, Trans. Dublin Soc., ser. 2, vol. iv, p. 160, fig.

Plymouth (A.M.N.).

\section{COPEPODA.}

\section{Calanidæ.}

Calanus finmarchicus (Gunnerus): G. O. Sars, Crust. Norway, vol. iv, p. 9, fig.

Plymouth, 1899, more or less common, Apr.-Aug., Oct. (P.т.C.) : $1903-4$, common in summer (L.H.G.).

\section{Paracalanidæ.}

Paracalanus parvus (Claus): G. O. Sars, Crust. Norway, vol. iv, p. 17 , fig.

Plymouth, 1888-9, in great abundance except from Sept.-Mar., when it appeared absent (G.C.B.): 1899; Mar.-Apr., fairly plentiful; June, very common; July-Aug., more or less rare; Sept.-Oct., more or less abundant; Nov., rare; Dec., more or less rare (P.T.c.): 1903, abundant all the year, except during May and July; 1904, abundant Jan.-July (L.H.G.). 
[Copepoda-contd.]

\section{Pseudocalanidæ.}

Pseudocalanus elongatus, Boeck: G. O. Sars, Crust. Norway, vol. iv, p. 20 , fig.

One of the commonest species, in immense numbers during autumn, winter, and spring, 1888-9 (G.C.B.): 1899, Feb.-Apr., more or less rare (P.T.C.) : very common in 1903 and 1904 (L.H.G.).

\section{Centropagidæ.}

Centropages typicus, Kröyer: G. O. Sars, Crust. Norway, vol. iv, p. 75 , fig.

Plymouth, 1888-9, abundant except during winter, when it is scarce (G.c.B.): 1899, Jan.-Feb., rare; Mar--Apr., in fair numbers; May, rare; June, more or less rare; July-Aug., rare; Sept., very rare (P.T.C.) : 1903, Jan.-Feb. ; Apr.-June; Aug.-Oct.; 1904, Jan.July (L.H.G.).

IsIAS ClaviPES, Boeck: G. O. Sars, Crust. Norway, vol. iv, p. 79, fig.

Plymouth, June 1899 (P.T.c.): Dec. 1902 ; Sept. 1903; Mar.June 1904 (L.H.G.).

\section{Temoridæ.}

Temora longicornis (O. F. Müller) : G. O. Sars, Crust. Norway, vol. iv, p. 97 , fig.

Plymouth, 1888-9; scarce during winter months, becoming more common in Apr., and reaching maximum abundance Aug.-Sept. (G.C.B.) : 1899 ; Mar., somewhat rare; Apr., very common ; JuneOct., rare (P.T.C.) : $1903-4$, rare in winter (L.H.G.).

\section{Pontellidæ.}

Anomalocera Patersoni, Templeton: G. O. Sars, Crust. Norway, vol. iv, p. 139, fig.

Plymouth, 1888-9, abundant in autumn and late summer, absent in winter (G.C.B.) : 1904, July (L.H.G.).

Labidocera Wollastoni, Lubbock: G. O. Sars, Crust. Norway, vol. iv, p. 142 , fig.

Plymouth; 1902, Dec.; 1904, Apr.-July (L.H.G.): near the Eddystone, in small numbers, Aug. and Oct., 1888 (G.c.B.).

\section{Parapontellidæ.}

Parapontella brevicornis (Lubbock): G. O. Sars, Crust. Norway, vol. iv, p. 145 , fig.

Near Eddystone, few specs. Sept. 1888; the Cattewater, Mar. 1889 (G.C.B.): Plymouth, rare, Mar.-Apr. 1899 (P.T.c.): 1903, MayJuly ; 1904, Feb.-May, July; rare in winter (L H.G.).

\section{Acartiidæ.}

Acartia Clausi, Giesbrecht: G. O. Sars, Crust. Norway, vol. iv, p. 150 , fig.

Plymouth, 1899; Jan., rare; Mar.-Apr., more or less abundant; June-Aug., common; Sept.-Oct., in fair numbers; Nov.-Dec., very rare (P.T.C.) : 1903, except during Mar. and May; 1904, Jan.-July (L.H.G.).

Acartia longiremis (Lilljeborg): G. O. Sars, Crust. Norway, vol. iv, p. 149 , fig.

One of the most common species at all seasons, 1888-9 (G.C.B.).

NEW SERIES, - VOL, VII. NO, 2 , 


\section{Longipediidæ.}

Longipedia Scotti: G. O. Sars, Crust. Norway, vol. v, p. 11, fig.

Plymouth (G.C.B.).

\section{Ectinosomidæ.}

Microsetella Norvegica (Boeck) [=Ectinosoma atlanticum, Brady]: G. O. Sars, Crust. Norway, vol. v, p. 44, fig.

Plymouth ; Jan.-Feb. 1899, rare (P.T.c.).

\section{Harpacticidæ.}

Euterpe acutifrons (Dana): C. Claus, Frei lebend. Copep., p. 110, fig. (as $E$. gracilis).

Plymouth, very abundant late winter and spring, 1889 (G.C.B.): 1899; Jan.-Feb., rare; Mar.-May, somewhat rare; July, fairly plentiful; Aug., rare; Sept., fairly common; Oct.-Dec., more or less rare (P.T.C.).

Thalestris mysis, Claus: G. S. Brady, Monogr. Free \& Semip. Copep. Brit. Is., vol, ii, p. 121, fig.

The Cattewater (G.c.B.).

\section{Cyclopidæ.}

Oithona plumifera, Baird: W. Giesbrecht, Fauna Flora Neapel, vol. xix, pp. 548, etc., fig.

Very abundant Feb.-Apr., practically absent during late summer and autumn, 1888-9 (G.C.B.).

Oithona similis, Claus.: $W$. Giesbrecht, Fauna Flora Neapel, vol. xix, pp. 548, etc., fig.

Plymouth, 1899, more or less common throughout the year (P.T.c.).

Cyclopina littoralis (Brady): G. S. Brady, Monogr. Free \& Semip.

Copep. Brit. Is., vol. i, p. 92, fig.

Plymouth, few specs. Apr. 1889 (G.c.b.).

\section{Corycæidæ.}

Corycaus anglicus, Lubbock: G. S. Brady, Monogr. Free \& Semip. Copep. Brit. Is., vol. iii, p. 34, fig.

Plymouth (G.C.B., P.T.C., T.V.H.).

Coryceus venustus, Dana: W. Giesbrecht, Fauna Flora Neapel, vol, xix, pp. 674 , etc., fig.

Plymouth, Nov. 1899, very rare (P.T.c.).

Oncma mediterranea (Claus): $W$. Giesbrecht, Fauna Flora Neapel, vol. xix, pp. 602, etc., fig.

Plymouth (G.C.B., P.T.C.).

Oncea minuta: $W$. Giesbrecht, Fauna Flora Neapel, vol. xix, pp. 603, etc., fig.

Plymouth, 1899, very rare Jan.-Mar., rare in Dec. (P.т.c.).

Oncæa subTILIS: $W$. Giesbrecht, Fauna Flora Neapel, vol. xix, pp. 603, etc., fig.

Plymouth, Mar. 1899, very rare (P.T.C.).

Saccopsis Alleni: É. Brumpt, C. R. Acad. Paris, vol. cxxiv, p. 1464, fig. Plymouth, on Polycirrus aurantiacus (E.B.). 
[Cirripedia: Leptostraca ]

\section{CIRRIPEDIA.}

Balanidæ.

Balanus balanoides (Linnæus): C. Darwin, Monogr. Cirrip., Balanidæ, p. 267 , fig.

Rocks between tide-marks in the Sound, etc. (s.P.).

Balanus crenatus, Bruguière: C. Darwin, Monogr. Cirrip., Balanidæ, p. 261 , fig.

Common in dredgings from the Sound, etc., on stones and shells (s.P.) : Eddystone Gds. (E.J.A.).

Balanus perforatus, Bruguière: C. Darwin, Monogr. Cirrip., Balanidæ, p. 271 , fig.

A bundant on rocks between tide-marks; rocks under the Laboratory; Millbay Dock, on piles; Drake's I.; etc. (s.P.).

Balanus spongicola, Brown: C. Darwin, Monogr. Cirrip., Balanidæ, p. 225 , fig.

Rame-Eddystone Gds., attached to the upper valve of Pecten opercularis; etc. (S.P.).

Pyrgoma anglicum, Leach: C. Darwin, Monogr. Cirrip., Balanidæ, p. 360 , fig.

Common on Caryophyllia Smithi, 15-35 fms. (R.A.T., S.P.).

Chthamalus stellatus (Poli): C. Darwin, Monogr. Cirrip., Balanidæ, p. 455 , fig.

Rocks between tide-marks in the Sound, etc. (S.P.).

Breeding: Jan.-Mar.; Aug.-Sept. (w.G.).

\section{Verrucidæ.}

Verruca Stroemia (O. F. Müller): C. Darwin, Monogr. Cirrip., Balanidæ, p. 518, fig.

Common on shells, stones, etc., L.W.-35 fms.; the Sound; Stoke Pt. Gds.; Rame-Eddystone Gds.; etc. (s.P.).

\section{Lepadidæ.}

Lepas anatifera, Linnæus: C. Darwin, Monogr. Cirrip., Lepadidæ, p. 73 , fig.

Occasionally on drift-wood, etc. (R.A.T., S.P.).

Scalpellum vulgare, Leach: C. Darwin, Monogr. Cirrip., Lepadidæ, p. 222 , fig.

Common on Halecium, Antennularia, Aglaophenia, etc., 15-35 fms. (R.A.T., S.P.).

Peltogastridæ.

Sacculina Carcini, Thompson: P. J. v. Beneden, Rech. Faune Litt. Belgique, Crust. [Bruxelles, 1861], p. 154, fig. Common, parasitic on Carcinus monas (S.P.). Breeding: May-Sept. (w.G.).

\section{LEPTOSTRACA.}

Nebalia BIPEs (Fabricius) : G. O. Sars, Fauna Norvegiæ, vol, i, p. 9, fig. Common under stones, at low tide; Drake's I.; Mt. Edgcumbe; Rum B., etc. (R.A.T., S.P.): Asia Sh., not uncommon (s.P.): Millbay Ch., occasionally; Whitsand B. (A.J.S.). Often abundant on the bait in lobster pots (E.J.A.).

Breeding: Apr.-July (w.G.). 


\section{AMPHIPODA.}

\section{Hyperiidæ.}

Hyperia Galba (Montagu): G. O. Sars, Crust. Norway, vol. i, p. 7, fig. Occasionally taken on Rhizostoma, Chrysaora, etc. (R.A.T.).

Parathemisto oblivia (Kröyer): G. O. Sars, Crust. Norway, vol. i. p. 10, fig.

Eddystone Gds. (G.C.B.).

\section{Orchestiidæ.}

Talitrus locusta (Pallas): G. O. Sars, Crust. Norway, vol, i, p. 23, fig. Abundant on all sandy shores, under weed and stones, at the high-water mark of spring tides (S.P.).

Orchestia LitTorea (Montagu): G. O. Sars, Crust. Norway, vol. i, p. 24, fig.

Abundant everywhere at high water, under weed and stones, and in rock crevices (s.P.).

Orchestia mediterranea, A. Costa: C. S. Bate \& Westwood, Brit. Sess.-eyed Crust., vol. i, p. 31, fig.

Drake's I.; Knap Buoy (т.v.H.).

\section{Lysianassidæ.}

Lysianax [= Lysianassa] Costæ (Milne-Edwards): G. O. Sars, Crust. Norway, vol. i, p. 42, fig.

Millbay Ch. (T.V.H. ${ }^{1}$ ).

Orchomene Batei, G. O. Sars: G. O. Sars, Crust. Norway, vol. i, p. 60 , fig.

Millbay Ch.; Queen's Gd. (T.V.H.).

Orchomenella nana (Kröyer): G. O. Sars, Crust. Norway, vol. i, p. 69, fig. (as 0 . ciliata).

Plymouth (T.V.H. $\left.{ }^{1}\right)$.

Tryphosa Sarsi (Bonnier): G. O. Sars, Crust. Norway, vol. i, p. 76, fig.

(as T. nana).

Queen's Gd. (T.V.H. ${ }^{1}$ ).

\section{Pontoporeiidæ.}

Bathyporeia pelagica, Spence Bate: G. O. Sars, Crust. Norway, vol, i, p. 129 , fig.

Whitsand B. (T.V.H. ${ }^{1}$ ).

\section{Ampeliscidæ.}

Ampelisca spinipes, Boeck: G. O. Sars, Crust. Norway, vol. i, p. 173, fig. Cawsand B., occasionally; Mewstone Gds., not uncommon on fine and medium gravel (R.A.T.).

Females bearing ova: Mar. (R.A.T.).

Ampelisca tenuicornis, Lilljeborg: G. O. Sars, Crust. Norway, vol, i, p. 167 , fig.

Duke Rk. (A,O.w. $\left.{ }^{1}\right)$ : near the Mewstone (A.M.N.). 
[Amphipoda-contd.]

\section{Amphilochidæ.}

Amphilochus manudens, Spence Bate: G. O. Sars, Crust. Norway, vol. i,

p. 217 , fig.

Inside Drake's I. (A.M.N., A.O.W.).

Amphilochoides odontonyx (Boeck): G. O. Sars, Crust. Norway, vol, i,

p. 221, fig.

Plymouth, 8 fms., 1887 (A.M.N.).

\section{Stenothoidæ.}

Stenothom marina (Spence Bate): G. O. Sars, Crust. Norway, vol. i, p. 236, fig.

Duke Rk. (A.O.w.1).

Stenothoe monoculoides (Montagu): G. O. Sars, Crust. Norway, vol, i, p. 240 , fig.

Plymouth (A.M.N.).

Stenothoe setosa: A. M. Norman, Ann. Mag. Nat. Hist., ser. 7, vol. vi, p. 39, fig.

Plymouth, single spec., the type (A.M.N.).

\section{Leucothoidæ.}

Leucothoe spinicarpa (Abildgaard): G. O. Sars, Crust. Norway, vol. i, p. 283, fig.

Millbay Ch., moderately common (T.V.H., R.A.T.): Cattewater; Queen's Gd.; Duke Rk. (т.V.H.): Yealm R., not uncommon (R.A.T.).

\section{Ediceridæ.}

Monoculodes carinatus, Spence Bate: G. O. Sars, Crust. Norway, vol. i,

p. 295, fig.

Plymouth (A.M.N.).

\section{Iphimediidæ.}

Iphimedia minuta, G. O. Sars: G. O. Sars, Crust. Norway, vol, i, p. 379 , fig.

Queen's Gd. (T.V.H., R.A.T.): Yealm R. (R.A.T.).

Iphimedia obesa, Rathke: G. O. Sars, Crust. Norway, vol. i, p. 377, fig. Plymouth Hr. (T.V.H. ${ }^{1}$ ).

\section{Calliopiidæ.}

Apherusa bispinosa (Spence Bate): G. O. Sars, Crust. Norway, vol. i, p. 439 , fig.

Inside Drake's I. (A.M.N. \& A.O.W. ${ }^{1}$ ): Yealm Estuary, very rare (R.A.T.).

\section{Atylidæ.}

Paratylus Swammerdami (Milne-Edwards): G. O. Sars, Crust. Norway, vol. i, p. 463, fig.

Cawsand B., not uncommon (R.A.T.): Rum B. (т.V.H.): Whitsand

B. (R.A.T.).

Females carrying ova: Jan., May (R.A.T.): Dec. (T.V.H.).

Dexamine spinosa (Montagu): G. O. Sars, Crust. Norway, vol. i, p. 475 , fig.

Inside Drake's I. (A.M.N. \& A.O.w.): Millbay; Cawsand B.; Knap Buoy; Whitsand B. (т.v.H.): Yealm Estuary, occasionally (R.A.T.). Breeding: July (R.A.T.). 
[Amphipoda-contd.]

\section{Gammaridæ.}

Gammarus Campylops, Leach: G. O. Sars, Crust. Norway, vol. i, p. 500, fig.

Queen's Gd. ${ }^{1}$; Duke Rk. ${ }^{1}$ (T.V.H.).

Gammarus locusta, Linnæus: G. O. Sars, Crust. Norway, vol. i, p. 499 , fig.

Very common under stones and among weeds, etc., between tidemarks to 5-6 fms. (R.A.T.).

Females with ova: Jan. (R.A.T.) : Dec. (R.A.T., т.v.H.).

Gammarus marinus, Leach: G. O. Sars, Crust. Norway, vol. i, p. 497, fig.

Common on the shore (R.A.T., S.P.) : Rum B. (R.A.T.): Drake's I.; the Breakwater; Mt. Edgcumbe; Wembury B. (т.V.H.): Reny Rks, (R.A.T.): Queen's Gd., occasionally (R.A.T., T.V.H., S.P.).

Females with ova: Feb. (R.A.T.): Mar. (т.v.H.).

Melita gladiosa, Spence Bate: T. R. R. Stebbing, Ann. Mag. Nat. Hist, ser. 4 , vol. v, p. 77 , fig.

Queen's Gd., occasionally (R.A.T.).

Melita obtusata (Montagu): A. M. Norman, Ann. Mag. Nat. Hist., ser. 6, vol. iv, p. 132.

Not uncommon, Millbay Ch. and Pit; Queen's Gd.; Cawsand B. (R.A.T.) : Duke Rk. (T.V.H.).

Females with ova: Jan., Apr., Dec. (R.A.T.).

Melita Palmata (Montagu): G. O. Sars, Crust. Norway, vol. i, p. 508, fig. Occasionally between tide-marks in the Sound (R.A.T.).

Mara grossimana (Montagu): C. S. Bate \& Westwood, Hist. Brit. Sess.-eyed Crust., vol, i, p. 350, fig. (o only); C. Heller, Denkschr. Akad. Wien, vol. xvi, p. 41, fig. ( $q$ only, as $M$. Donato $i$ ).

Occasionally taken between tide-marks (R.A.T.): Bovisand Cove (J.T.C.).

Mera Othonis (Milne-Edwards): G. O. Sars, Crust. Norway, vol. i, p. 518, fig.

Millbay Ch., occasionally (R.A.T.): Duke Rk. ${ }^{1}$; Yealm R. ${ }^{1}$ (т.v.H.).

Mara semiserrata (Spence Bate): C. S. Bate \& Westwood, Hist. Brit.

Sess.-eyed Crust., vol. i, p. 401, fig. (as Megamara).

Nr. Eddystone, 30 fms. (A.O.w.).

Gammarella brevicaudata (H. Milne-Edwards): T. R. R. Stebbing, Ann. Mag. Nat. Hist., ser. 4, vol. xiv, p. 13, fig.

Plymouth (A.O.W.' ${ }^{1}$ ).

Cheirocratus Sundewalli (Rathke): G. O. Sars, Crust. Norway, vol. i, p. 524, fig.

The Sound, occasionally (R A.T.).

\section{Photidæ.}

Aora Gracilis (Spence Bate): G. O. Sars, Crust. Norway, vol. i, p. 545, fig. The Sound, occasionally (R.A.T.).

Gammaropsis ERYthrophthalma (Lilljeborg): G. O. Sars, Crust. Norway, vol. i, p. 558, fig.

Millbay Ch. (T.V.H.). 
[ Amphipoda-contd.]

\section{Podoceridæ.}

Amphithoe rubricata (Montagu); G. O. Sars, Crust. Norway, vol. i, p. 579, fig.

Not uncommon in dredgings from the Sound and occasionally between tide-marks (R.A.T.): Yealm R. (т.v.H.).

Female carrying young: Feb. (R.A.T.).

Ischyrocerus minutus, Lilljeborg: G. O. Sars, Crust, Norway, vol. i, p. 589, fig.

Duke Rk., 5 fms. (T.v.H. ${ }^{1}$ ).

Podocerus falcatus (Montagu): G. O. Sars, Crust. Norway, vol. i, p. 594, fig.

Abundant on the buoys, hulks, and piles in the Sound, Millbay Docks, and Cattewater (R.A.T.).

A female with ova: Nov. (т.v.H.).

Erichthonius abditus (Templeton): G. O. Sars, Crust. Norway, vol. i, p. 602 , fig.

Millbay Ch.; Queen's Gd.; common (R.A.T., S.P.): Yealm R. (R.A.T.) : Eddystone Gds. (E.J.A.).

\section{Corophiidæ.}

Corophium Bonelli, Milne-Edwards: G. O. Sars, Crust. Norway, vol. i, p. 616 , fig.

Millbay ${ }^{1}$; Queen's Gd. ${ }^{1}$; Duke Rk. ${ }^{1}$ (T.V.H.).

Corophium crassicorne, Bruzelius: G. O. Sars, Crust. Norway, vol. i, p. 615 , fig.

Millbay Ch. and Pit, very common; Queen's Gd., not uncommon (R.A.T., т.V.H.): West Ch. : Yealm R. (т.V.H.).

Females with ova: Nov. (т.v.H.).

Unciola crenatipalma (Spence Bate): C. S. Bate \& Westwood, Hist. Brit. Sess.-eyed Crust., vol, i, pp. 488, 489, fig. (o as Dryope irrorata, o as $D$. crenatipalmata).

Plentiful among stones and shells on muddy bottom, $20 \mathrm{fms}$. (W.G., A.M.N.).

\section{Cheluridæ.}

Chelura terebrans, Philippi: G. O. Sars, Crust. Norway, vol. i, p. 627 , fig.

Common in drift-wood (R.A.T., S.P.).

\section{Caprellidæ.}

Phtisica marina, Slabber: G. O. Sars, Crust. Norway, vol. i, p. 646, fig. Inside Drake's I. (E.J.A.): N. of the Breakwater (T.v.H. ${ }^{1}$ ): Whitsand B., single spec. (J.T.C.): Eddystone Gds. (E.J.A.).

Protella phasma (Montagu): G. O. Sars, Crust. Norway, vol. i, p. 649, fig. Moderately common, Millbay Ch.; Asia Sh.; Drake's I.; Queen's Gd.; Yealm R. (R.A.T.): 4 miles W. of the Eddystone, single spec. (R.A.T.).

Females with ova: Mar.-Apr. (R.A.T.).

Pariambus typicus (Kröyer): G. O. Sars, Crust. Norway,vol. i, p. 655, fig.

Cawsand B.; Jennycliff B. (т.V.H.). 
[Aphipoda-contd. : Isopoda ]

Caprella acanthifera, Leach: G. O. Sars, Crust. Norway, vol. i, p. 666, fig.

Millbay Ch.; Eddystone, on sponge (т.v.н.).

CAPrella equUllibra, Say: G. O. Sars, Crust. Norway, vol. i, p. 663, fig. The Sound, common on buoys and hulks (R.A.T.).

Caprella linearis (Linnæus) : G. O. Sars, Crust. Norway, vol. i, p. 657, fig.

Drake's I., at low water (J.T.C. $\left.{ }^{1}\right)$ : Millbay Ch. ${ }^{1}$; Queen's Gd. ${ }^{1}$; Yealm R. ${ }^{1}$ (T.V.H.).

Caprella tuberculata, Guerin: C. S. Bate \& Westwood, Hist. Brit.

Sess.-eyed Crust., vol. ii, p. 68 , fig.

Occasionally in the Sound (R.A.T.).

\section{ISOPODA.}

Apseudidæ.

Apseudes Latreillei (Milne-Edwards): A. M. Norman \& Stebbing, Trans. Zool. Soc., vol. xii, p. 82, fig.

Drake's I., common among mud at roots of corallines (w.G.): common under stones and in rock crevices; Jennycliff B.; Drake's I. (S.P.).

Females carrying ripe ova in Aug. (s.P.).

Apseudes talpa (Montagu): A. M. Norman \& Stebbing, Trans. Zool. Soc., vol. xii, p. 81.

Not uncommon between tide-marks; Rum B.; Reny Rks.; Wembury B. (R.A.T.): Millbay Ch. (R.A.T.).

Ova hatching: Feb. (R.A.T.).

\section{Tanaidæ.}

Tanais tomentosus, Kröyer: G. O. Sars, Crust. Norway, vol. ii, p. 12 , fig.

Common in crevices of the limestone of Plymouth Breakwater (R.A.T.).

\section{Anthuridæ.}

Anthura gracilis (Montagu): A. M. Norman \& Stebbing, Trans. Zool. Soc., vol. xii, p. 122 , fig.

Occasional specimens in dredgings from the Sound; Asia Sh.; Queen's Gd.; Millbay Ch. (R.A.T.).

\section{Gnathiidæ.}

Gnathia maxillaris (Montagu): G. O. Sars, Crust. Norway, vol. ii, p. 52, fig.

Common under stones between tide-marks, and in dredgings from the Sound (R.A.T., S.P.): Mewstone Gds. (R.A.T.).

Females with ova: Jan.; Feb. (R.A.T.): March (s.P.): May (R.A.T.). Hatching out: Feb. (R.A.T.).

\section{Ægidæ.}

Rocinela damnoniensis, Leach: J. C. Schicedte \& Meinert, Naturhist.

Tidsskr., ser. 3, vol. xii, p. 383, fig.

Occasional specs., $20-30$ fms. (R.A.T., S.P.).

Breeding: Feb. (R.A.T.). 
[ Isopoda-contd.]

Rocinela Dumerili (Lucas): J. C. Schicedte \& Meinert, Naturhist.

Tidsskr., ser. 3, vol. xii, p. 391, fig.

Occasional specs., $20-30$ fms. (R.A.T., S.P.).

Hatching out: Aug. (R.A.T.).

\section{Cirolanidæ.}

Cirolana hirtipes, H. Milne-Edwards: H. J. Hansen, Danske vid. Selsk. Skr., ser. 6, Naturvid., vol. v, p. 326, fig.

Drake's I. (A.0.W.).

Conilera cylindracea (Montagu): H. J. Hansen, Danske vid. Selsk. Skr., ser. 6, Naturvid., vol. v, p. 358, fig.

West Entrance; Mewstone Ledge; occasionally (R.A.T.): Mewstone Gds, not uncommon (R.A.T., S.P.): Stoke Pt. Gds. (S.P.); Rame-Eddystone Gds. (R.A.T., S.P.).

Breeding: Apr. (R.A.T.): June-Aug. (S.P.).

A dead, but fresh specimen of Scyllium canicula brought to the Laboratory in Sept. 1899, contained about 400 C. cylindracea, of which over 300 were living; having eaten through the wall of the stomach of the dogfish, they were feeding on the heart and liver (R.A.T.).

\section{Cymothoidæ.}

Nerocila neapolitana: J. C. Schicedte \& Meinert, Naturhist. Tidsskr., ser. 3 , vol. xiii, p. 41 , fig.

Single spec., 5-6 m. S. of the Mewstone (A.M.N.).

Breeding: Sept. (A.M.N.).

Dynamene rubra, Leach: C. Spence Bate \& Westwood, Brit. Sess.-eyed Crust., vol. ii, p. 419, fig.

Rum B., common; Reny Rks., occasionally (R.A.T.).

Breeding: Feb. (R.A.T.).

\section{Sphæromidæ.}

Nesa bidentata (Adams): C. Spence Bate \& Westwood, Brit. Sess.-eyed Crust., vol. ii, p. 431, fig.

Not uncommon in empty barnacle shells, etc., between tide-marks in the Sound (R.A.T.): Jennycliff B., abundant in rock crevices; Mt. Edgcumbe, under stones; the Breakwater; etc. (s.P.).

Spharoma curtum, Leach : C. Spence Bate \& Westwood, Brit. Sess.-eyed Crust., vol. ii, p. 412, fig.

Drake's I. (A.o.W.).

Spharoma serratum (Fabricius): C. Spence Bate \& Westwood, Brit. Sess.-eyed Crust., vol. ii, p. 405, fig.

Under stones; Drake's I.; Rum B. (R.A.T., S.P.).

\section{Limnoriidæ.}

Limnoria lignorum (Rathke): G. O. Sars, Crust. Norway, vol. ii, p. 76, fig. Common in drift wood, etc., in the Sound (R.A.T., S.P.).

\section{Idotheidæ.}

IdotheA Baltica (Pallas) [=I. tricuspidata, Desmarest]: G. O. Sars, Crust. Norway, vol. ii, p. 80, fig.

Queen's Gd., on Antennularia ${ }^{1}$; Cawsand B., on Zostera; Whitsand B. (s.P.). 
Idothea emarginata (Fabricius): G. 0. Sars, Crust. Norway, vol. ii, p. 85 , fig.

Jennycliff B., common (т.V.H., R.A.T.): Whitsand B. (s.P.).

Idothea linearis (Linnæus): C. Spence Bate \& Westwood, Brit. Sess.eyed Crust., vol. ii, p. 388, fig.

Common among Zostera; Drake's I.; Jennycliff B.; Cawsand B.; Whitsand B.; Yealm Est. (s.P.).

Idothea Neglecta : G. O. Sars, Crust. Norway, vol. ii, p. 84, fig.

Drake's I., abundant under stones at low-water; Jennycliff B., Mt. Edgcumbe, etc., occasionally (s.P.).

Breeding: Mar. (s.P.).

Idothea prismatica (Risso): C. S. Bate \& Westwood, Brit. Sess.-eyed Crust., p. 391, fig. (as I. parallela).

Cawsand B., single spec. inhabiting a dead Zostera stem (w.G.).

IdotheA PELAGicA, Leach: G. O. Sars, Crust. Norway, vol. ii, p. 81, fig.

Single spec., between Breakwater Lt. and Queen's Gd. Buoy (J.T.C.). Carrying embryos: Dec. (J.T.C.).

\section{Arcturidæ.}

Astacilla longicornis (Sowerby): G. O. Sars, Crust. Norway, vol. ii, p. 88 , fig.

Not uncommon elinging to the spines of Echinus esculentus; Eddystone Gds. (R.A.T., S.P.): Mewstone Ledge, occasionally (R.A.T.). Breeding: Apr. (R.A.T.).

Astacilla intermedia (Goodsir): C. Spence Bate \& Westwood, Brit. Sess.-eyed Crust., vol. ii, p. 371, fig. (as Arcturus).

The Breakwater (w.н.): Queen's Gd. (т.v.н.).

Janiridæ.

Janira Maculosa, Leach: G. O. Sars, Crust. Norway, vol. ii, p. 99, fig. Common in Millbay Pit and between tide-marks at Mt. Edgcumbe and Drake's I.; less common in dredgings from Queen's Gd. and Asia Sh. and on the Reny Rks.; occasionally outside in 33 fms. (R.A.T.).

Females carrying ova: Mar.-Apr. (R.A.T.).

J ERA Marina (Fabricius) $[=J$. albifrons, Leach]: G. O. Sars, Crust. Norway, vol. ii, p. 104, fig.

Rocks under the Hoe; Queen's Gd.; the Breakwater; not uncommon (R.A.T.): Hollow Rock B., common under stones at lowwater (s.P.): Reny Rks., common (R.A.T.).

Breeding: Feb. (R.A.T.).

\section{Munnidæ.}

MunNa KröYeri, Goodsir: G. O. Sars, Crust. Norway, vol. ii, p. 109, fig. Millbay Ch.; Asia Sh.; Queen's Gd.; Yealm R.; not uncommon (R.A.T.).

\section{Ligiidæ.}

Ligia oceanica (Linnæus): G. O. Sars, Crust. Norway, vol. ii, p. 156, fig. Common in rock-crevices, etc., above high-water mark in the Sound (R.A.T., S.P.): abundant on quay walls in the Cattewater (S.P.) : the Breakwater (A.O.W., S.P.).

Breeding: June (A.J.S.). 


\section{Bopyridæ.}

Bopyrus Squillarum, Latreille: G. O. Sars, Crust. Norway, vol. ii, p. 197 , fig.

Not uncommon on Leander serratus; Cawsand B. ; etc. (R.A.T.).

Gyge Galathes: C. S. Bate \& Westwood, Brit. Sess.-eyed Crust., p. 225 , fig.

Rum B., on Galathea squamifera (R.A.T.).

\section{Cryptoniscidæ.}

Cryptothir Balani, Spence Bate: G. O. Sars, Crust. Norway, vol. ii, p. 236, fig.

Rocks below the Hoe, not uncommon among barnacles (R.A.T.).

\section{CUMACEA.}

\section{Cumidæ.}

I phinoe trispinosa (Goodsir): G. O. Sars, Crust. Norway, vol. iii, p. 14, fig. Abundant 1892 (w.G.): Whitsand B., May 1902, May 1903(A.M.N.).

\section{Leuconidæ.}

Eudorella truncatula, Spence Bate: G. O. Sars, Crust. Norway, vol. iii, p. 37, fig.

Cawsand B.; 1889 ; Apr. 1900 ; June 1903 (A.M.n.).

\section{Diastylidæ.}

Diastylis Bradyi: A. M. Norman, Ann. Mag. Nat. Hist., ser. 5, vol.iii,p. 59.

Cawsand B., Apr. 1900; Whitsand B., May 1902, and common in May 1903 (A.M.N.).

Diastylis laevis $[?=$ D. rostrata (Goodsir)]: A. M. Norman, Ann. Mag. Nat. Hist., ser. 5, vol. iii, p. 60.

Sept. 1889 ; 3 m. N.E. of the Eddystone, Sept. 1900 (A.M.N.).

Diastylis Rathkei (Kröyer): G. O. Sars, Crust. Norway, vol. iii, p. 44, fig.

Whitsand B. (A.J.S.).

\section{Pseudocumidæ.}

Pseudocuma cercaria (P. J. v. Beneden): G. O. Sars, Crust. Norway, vol. iii, p. 74 , fig.

Cawsand B., Apr. 1900; Whitsand B., May 1902, and abundant in May 1903 (A.M.N.).

\section{STOMAPODA.}

Squilla Desmaresti, Risso: T. Bell, Hist. Brit. Stalk-eyed Crust., p. 354, fig.

Single spec. near New Ground Buoy, Dec. 1900 (R.A.T.).

The larvæ are occasionally met with in townettings (E.J.A.).

\section{SCHIZOPODA.}

\section{Euphausiidæ.}

Nyctiphanes Couchi : T. Bell, Hist. Brit. Stalk-eyed Crust., p. 346, fig. (as Thysanopoda).

Occasionally taken in the townets, in the Sound and outside (R.A.T.): single spec., 3 m. off Rame Hd., June 1898 (E.w.L.H.): Cawsand B. and $2 \frac{1}{2} \mathrm{~m}$. S. of Breakwater, few immature specs., Feb. 1899 (w.I.B.). 


\section{Siriellinæ.}

[Schizopoda-contd.]

Siriella armata (Milne-Edwards): G. O. Sars, Arch. Math. Naturvid., vol. i, p. 96, fig.

June 1893, Drake's I. (w.G.): Cawsand B., few specs. Apr., May, June, Sept. 1899, Apr. 1900; Jennycliff B., few specs. Sept. and Oct. 1899; Whitsand B., abundant Aug. 1898 and Oct. 1899; Bovisand B., Oct. 1899; Yealm Est., Oct. 1899 and Apr. 1900 (w.I.B.).

Breeding: Apr.; Oct. (W.I.B., R.A.T.).

Siriella Clausi: G. O. Sars, Arch. Math. Naturvid., vol. i, p. 81, fig.

Cawsand B., few specs. Apr. June, and Sept.; Jennycliff B., few specs., June and Oct.; Yealm Est., several specs. among decaying Zostera, Oct.; $2 \frac{1}{2}$ m. S. of the Breakwater Fort, single spec. at surface, Feb. ; 1899 (w.I.B.).

Siriella Jaltensis, Czerniavsky: G. O. Sars, Arch. Math. Naturvid., vol. i, p. 89, fig. (as $S$. crassipes).

At the surface $1 \mathrm{~m}$. S. of the Breakwater Fort, Sept. 1892; Drake's I., abundant among weeds June 1893 (w.G.): Rum B., single spec. Feb. 1901 (R.A.T.) : Jennycliff B. (F.w.G. \& W.I.B.).

Breedirg: June (W.G., R.A.T.).

\section{Gastrosaccinæ.}

Gastrosaccus sanctus (v. Beneden): G. O. Sars, Arch. Math. Naturvid., vol. i, p. 56, fig.

Whitsand B., Aug. 1892 (w.G.): July 1898 (E.w.L.H.): Oet. 1899, abundant (w.I.B.): May 1903, abundant (s.P.): Cawsand B., immature spec. in Apr.; Yealm Est., single spec. among decaying Zostera, Oct.; 1899 (w.I.B.).

Breeding: July (E.w.L.H.): Aug. (w.G.): Oct. (w.I.B., R.A.T.). Hatching out: May (R.A.T.).

Haplostylus Normani: G. O. Sars, Arch. Math. Naturvid., vol. i, p. 65, fig. (as Gastrosaccus).

At the surface about $1 \mathrm{~m}$. S. of the Breakwater Fort, Sept. 1892 (w.G.): Mewstone 'Amphioxus' Gd., Oct. 1899 (w.I.B.).

Breeding: Sept. (w.G.): Oct. (w.I.B.)

Anchialis agilis: G. O. Sars, Arch. Math. Naturvid., vol. i, p. 70, fig.

Plymouth, 1889; 1890 (A.M.N.): Aug. 1898, 3 m. S. of Rame Hd. (E.w.L.H. \& w.I.B.) : 1899 ; Cawsand B., few specs. in Feb. ; Mewstone 'Amphioxus' Gd., several in Oct.; W. of Hand Deeps, $33 \mathrm{fms}$., sand, abundant in June; about $4 \mathrm{~m}$. S. of the Breakwater, abundant in Mar.; etc. (w.I.B.): Whitsand B., single spec., May 1903 (s.P.).

Breeding: June (w.I.B., R.A.T.).

\section{Heteromysinæ.}

Heteromysis formosa, S. I. Smith: A. M. Norman, Ann. Mag. Nat. Hist., ser. 6, vol. x, p. 158, fig.

Millbay Ch.; Queen's Gd.; Cawsand B.; Yealm R.; off Stoke Pt.; a few at a time (w.G.): Jan. 1899, Millbay Ch.; Feb. 1900, Asia Sh. (w.I.B.).

Breeding: Oct.-Nov. (w.G.). 


\section{Leptomysinæ.}

Erythrops elegans (G. O. Sars): G. O. Sars, Carcinol. Bidr. Norges Fauna, Mysider, Pt. i, p. 33, fig. (as E. pygmoea). 3 m. S.W. of Rame Hd., Aug. 1898 (e.w.L.H. \& w.I.B.): RameEddystone Gds.; Mewstone 'Amphioxus' Gds.; etc.; several specs. Mar., June, Oct. and Nov. 1899 (w.I.B.). Breeding: June; Oct. (w.I.B.): Oct., late embryos only (w.G.).

Mysidopsis angusta, G. O. Sars: G. O. Sars, Carcinol. Bidr. Norges Fauna, Mysider, Pt. ii, p. 27, fig. Occasionally in 15-33 fms. (R.A.T.): W. of Hand Deeps, June; 4 m. S.S.W. of Rame Hd., and Mewstone 'Amphioxus' Gd., Oct.; $1 \mathrm{~m}$. W. of Stoke Pt., Nov.; 1899 (w.I.B.). Breeding: June-Oct. (W.I.B., R.A.T.).

Mrsidopsis gibbosa, G. O. Sars: G. O. Sars, Carcinol. Bidr. Norges Fauna, Mysider, Pt. ii, p. 23, fig.

Cawsand B., several occasions 1893-4 (w.g.): Aug. 1898, few specs. 3 m. S.W. of Rame Hd. (E.W.L.H. \& w.I.B.) : 1899; single spec., Cawsand B. in Apr.; three specs. W. of Hand Deeps in June (w.I.B.): Apr. 1902, Jennycliff B. (F.w.G.).

Breeding: Apr. (w.I.B., R.A.T.) : July (w.G.).

Leptomysis gracilis (G. O. Sars): G. O. Sars, Carcinol. Bidr. Norges Fauna, Mysider, Pt. iii, p. 31, fig.

Aug. 1896, 3 m. W. by S. of the Eddystone (W.I.B.): Aug. 1898,

3 m. S.W. of Rame Hd. (E.W.L.H. \& W.I.B.): June 1899, fairly abundant W. of Hand Deeps (w.I.B.).

Breeding: June (W.I.B., R.A.T.).

Leptomysis lingvura (G. O. Sars): G. O. Sars, Carcinol. Bidr. Norges Fauna, Mysider, Pt. iii, p. 35, fig.

Cawsand B.; Rame Hd., on the shore among Fucus; Whitsand

B.; 4 m. S.S.W. of Rame Hd.; W. of Hand Deeps, on sand; Yealm

Est., among decaying Zostera; several specs., Feb.-Oct. 1899 (W.I.B.). Breeding: Apr.; Oct. (w.I.B., R.A.T.).

Leptomysis mediterranea: G. O. Sars, Arch. Math. Naturvid., vol. i, p. 37, fig.

Cawsand B.: common June 1893 (w.G.): Aug. 1898 (E.w.L.H. \& w.I.B.): several specs. Feb., Apr., May, June, Sept. Oct. 1899 (w.I.B.). Jennycliff B. : single specs. Oct. and Dec. 1899 (w.I.B.): Apr. 1902, single spec. (R.A.T.). Bovisand B.; Whitsand B.; Yealm Est., among decaying Zostera; Oct. 1899 (w.I.B.).

Breeding: Apr. (w.I.B., R.A.T.): June (w.G.): Oct.; Dec. (w.I.B., R.A.T.).

\section{Mysinæ.}

Hemimysis Lamorna (Couch): G. O. Sars, Carcinol. Bidr. Norges Fauna, Mysider, Pt. iii, p. 65, fig. (as Mysis).

Cawsand B.: July 1893 (w.G.): a doubtful spec. Apr. 1900 (w.I.B.). Drake's I., single spec. (R.A.T.). 
[Schizopoda-contd.]

Macromysts flexuosa (Müller) and M. nigra: F. Keeble \& Gamble, Phil. Trans. Roy. Soc., ser. B., vol. exevi, pp. 332, etc., fig.

Together these two species include, but they do not respectively coincide with, the Macromysis flexuosa and $M$. neglecta of Sars and of Norman. Both are known to occur in the Plymouth area, but they have only been quite lately differentiated, and consequently their distribution has not yet been fully determined. The evidence at present available points to the conclusion that $M$. flexuosa is essentially an estuarine form, and $M$. nigra more strictly marine. This is in agreement with the experience of Gamble. The Macromysis which swarms about the shores of the Tamar at Saltash (especially in the summer months) appears to be exclusively $M$. Alexuosa. In Plymouth Sound $M$. nigra is the predominant form; it occurs commonly in the shallows, especially in Cawsand Bay; but $M$. flexuosa has been found in small numbers also. Both species are known to occur in the R. Yealm. M. nigra has been found in Whitsand Bay once (w.I.B.).

Macromysis Inermis (Rathke): G. O. Sars, Carcinol. Bidr. Norges Fauna, Mysider, Pt. iii, p. 54, fig.

Plymouth, 1889 (A.M.N.): Sept. 1892 (w.G.): 1898; Cawsand B., Bigbury B. in July (w.I.B.): 1899; Cawsand B.; Jennycliff B.; Bovisand B.; Whitsand B.; Yealm Est. among decaying Zostera few specs., Feb.-Oct. : Apr. 1900 (w.I.B.).

Breeding: Apr.; Oct. (w.I.B.).

Schistomysis arenosa: G. O. Sars, Arch. Math. Naturvid., vol. i, p. 16, fig. (as Mysis).

Cawsand B.: 1893 (w.G.): Feb. 1899, four specs.; Apr. 1900, single spec. (w.I.B.). Whitsand B.: $1892-3$ (w.G.): 1896 (т.v.H.): Oct. 1899 (w.I.B.): May 1903, several specs. (s.P.). Bovisand B. : Yealm Est., on sand with decaying Zostera; Oct. 1899 (w.I.B.).

Breeding: June (w.G.): Oct. (W.I.B., R.A.T.).

Schistomysis ornata (G. O. Sars): G. O. Sars, Carcinol. Bidr. Norges Fauna, Mysider, Pt. iii, p. 62, fig. (as Mysis).

R. Tamar, Saltash: Mar.; Dec.; 1899 (w.I.B.): Feb. 1901 (w.I.B. \& R.A.T.). 3 m. S.W. of Rame Hd., few specs. Aug. 1898 (E.w.L.H. \& W.I.B.): Rame-Eddystone Gds.; Mewstone Gds. etc.; several specs. Mar.-Nov. 1899 (w.I.B.).

Breeding: Feb.; Apr.; Oct. (w.I.в., R.A.T.).

Schistomysis Parkeri: A. M. Norman, Ann. Mag. Nat. Hist., ser. 6, vol. $\mathrm{x}, \mathrm{p} .256$, fig.

Whitsand B., 1896 (т.v.H.): Tregantle, 1897 (E.w.L.H.).

Schistomysis spiritus, Norman: G. O. Sars, Carcinol. Bidr. Norges Fauna, Mysider, Pt. iii, p. 58, fig. (as Mysis).

Whitsand B.: abundant July 1892 (w.G.): 1897 (E.W.L.H.): Aug. 1898, abundant (E.W.L.H. \& w.I.B.): May 1903 (s.P.). Cawsand B., single specs. in Feb. and Apr. 1899 (w.I.B.). 3 m. S.W. of Rame Hd., Aug. 1898 (E.w.L.H. \& W.I.B.).

Breeding: Apr. (w.I.B., R.A.T.): June; July; Aug., late stages only (w.G.). Large individuals almost disappeared by Aug., young ones numerous (w.G.). 
[Schizopoda-contd.: Macrura]

Neomysis vulgaris (Thompson): G. O. Sars, Carcinol. Bidr. Norges Fauna, Mysider, Pt. iii, p. 80, fig. (as Mysis).

Laira, 1897 (E.W.L.H.): R. Lynher; tidal pool opposite Saltash ; Summer 1898 (E.W.L.H. \& w.I.B.): R. Tamar, just above Saltash Bridge, few specs. Mar. and Dec. 1899; Jennycliff B., single young spec. Sept. 1899 (W.I.B.): Saltash, Feb. 1901 (R.A.T.).

Breeding: Feb. (R.A.T.).

Dasymysis Longicornis (Milne-Edwards): G. O. Sars, Arch. Math. Naturvid., vol. i, p. 22, fig. (as Mysis).

W. of Hand Deeps, June $1899 ; 4$ m. S.S.W. of Rame Hd., Oct. 1899 ; several spees. (W.I.B.).

Breeding: June; Oct. (W.I.B.).

Macropsis Slabberi (v. Beneden): G. O. Sars, Arch. Math. Naturvid., vol. i, p. 28 , fig.

Saltash, very abundant Mar. and Dec. 1899; Cawsand B., single spec. Apr. 1900 (w.I.B.): Tregantle, single spec. Dec. 1897 (E.W.L.H.): Whitsand B., several spees. May 1903 (s.P.).

\section{MACRURA.}

\section{Palæmonidæ.}

Leander serratus (Pennant): T. Bell, Hist. Brit. Stalk-eyed Crust., p. 302, fig. (as Palamon).

More or less common everywhere among weed (R.A.T., S.P.).

Breeding: Jan.-Feb. (w.G., R.A.T.) : Mar.-Apr. (w.G., R.A.T., s.P.) : May (w.G., R.A.t.) : June (w.G.): Nov. (W.G., R.A.T.): Dec. (W.G.).

Leander squilla (Linnæus): T. Bell, Hist. Brit. Stalk-eyed Crust., p. 305, fig. (as Palcemon).

The Sound, not uncommon between tide-marks (R.A.T.).

Breeding: May (R.A.T.): July, and earlier (w.G.). Hatehed out in Aug. (R.A.T.).

Palmononetes varians (Leach): T. Bell, Hist. Brit. Stalk-eyed Crust., p. 309, fig. (as Palcemon).

Common in brackish water; Saltram; Laira; etc. (R.A.T.): Erth, R. Lynher, common in pools on a salt-marsh (W.I.B): tributaries of R. Plym, very common (w.F.R.w.).

Breeding: Apr. (R.A.T.): May-June (w.G., R.A.T.): July; Aug., late stages only (w.G.).

\section{Pandalidæ.}

Pandalus Montagui, Leach: T. Bell, Hist. Brit. Stalk-eyed Crust., p. 297, fig. (as P. annulicornis).

Occasionally abundant during short periods, at other times apparently quite absent (s.P.): Nov. 1887, large numbers in Batten B. (w.н.): Feb. 1896, single spec. Cawsand B. (т.v.н.): Aug. 1897; Sept. 1898 (E.W.L.H.): July-Aug. 1902 ; Aug. 1903 (S.P.).

Breeding: Jan. (w.G.): Feb. (w.G., T.v.H., R.A.T.): Nov. (w.G., R.A.T.): Dee. (w.G.). 
[Macrura-contd.]

Pandalus brevirostris, Rathke: C. Heller, Crust. südl. Europa, p. 247, fig. Common in the Sound, especially on clean coarse gravel and clinker grounds, 5-10 fms.; common on 'Cellaria' grounds, 15-20 fms. (R.A.T.).

Females in berry: Mar. (R.A.T., s.P.): Apr. (w.G., R A.T.): MayJuly (R.A.T.). Zoæa stage: Mar.-June (R.G.).

\section{Hippolytidæ.}

Hippolyte fascigera, Gosse: A. Wolleboek, Rept. Norweg. Fish. \& Mar.

Invest., vol. i, No. 4, p. 27 (as Virbius).

Cawsand B. (R.A.T.).

Breeding: Apr. (R.A.T.).

Hippolyte varians, Leach: C. Heller, Crust. südl. Europa, p. 288, fig. (as Virbius).

More or less common everywhere among weed, L.W.-5 fms.; occasionally taken outside in 15-30 fms. (R.A T.).

Breeding: Feb. (R.A.T.): Mar. (R.A.т., s.P.): Apr. (R.A.т.): MayJuly (w.G., R.A.T.): Aug.; Nov. (R.A.T.).

Hippolyte viridis (Otto): C. Heller, Crust. südl. Europa, p. 286, fig. (as Virbius).

Yealm Est., not uncommon (R.A.T.).

Breeding: July (R.A.T.).

Spirontocaris Cranchi (Leach): T. Bell, Hist. Brit. Stalk-eyed Crust., p. 288, fig. (as Hippolyte).

Moderately common in the Sound, L.W.-25 fms, and on the outside grounds, $15-30$ fms. (R.A.T.).

Breeding: Feb. (w.H.): Apr. (w.G., R.A.T.): May (w.G.): June; Aug. (R.A.T.). A female spawned in a Lab. tank in Apr. (R.A.T.).

\section{Alpheidæ.}

Alpheus ruber, Milne-Edwards: T. Bell, Hist. Brit. Stalk-eyed Crust., p. 271, fig.

Single spec., Church Reef, Wembury B., under a stone (R.A.T.).

Athanas nitescens (Leach): T. Bell, Hist. Brit. Stalk-eyed Crust., p. 281, fig.

Not uncommon in rock-pools and among corallines, Fucus, etc. (S.P.) : generally gregarious, $3-4$ specs. under one stone (R.A.T.) : Rum B.; Mt. Edgeumbe; Wembury B. (R.A.T., s.P.): Tinside (R.G., R.A.T.): Cawsand B., occasionally between tide-marks; occasional specs, on the outside grounds, $15-30$ fms. (R.A.T.).

Breeding: May (R.G.): July (s.P.): Aug. (R.A.T.).

\section{Crangonidæ.}

Crangon Allmanni, Kinahan: A. Wolleboe, Rept. Norweg. Fish. \& Mar. Invest., vol. i, No. 4, p. 21, fig.

Very rare, 15-30 fms. (R.A.T.): 3 m. S.S.W. of Rame Hd., common Aug. 1898 (w.I.B.)

Crangon vulgaris (Linnæus): T. Bell, Hist. Brit. Stalk-eyed Crust., p. 256 , fig.

More or less abundant everywhere on sand, L.W.-10 fms. (R.A T., S.P.).

Breeding: Jan.-Aug. (w.G., R.A.T.) : Nov. (R.A.T.): Dec. (w.G.). 
[ Macrura-contd.]

Egeon fasciatus, Risso: T. Bell, Hist. Brit. Stalk-eyed Crust., p. 259, fig. (as Crangon).

Rum B.; Jennycliff B.; Cawsand B.; sand-patch N. of Drake's I.; not uncommon, L.W.-5 fms. (R.A.T.): West Entrance (J.T.C.).

Breeding: Mar.-Apr. (R.A.т.) : May (w.G.). Hatching out: Apr. (R.A.T.). Zoæas: May; July-Sept. (R.G.).

Egeon sculptus: T. Bell, Hist. Brit. Stalk-eyed Crust., p. 263, fig. (as Crangon).

Occasionally, 15-30 fms. (R.A.T.).

Breeding: June (w.G.) : Aug. (R.A.T.).

Pontophilus spinosus, Leach: T. Bell, Hist. Brit. Stalk-eyed Crust., p. 261, fig. (as Crangon).

Mewstone Gds.; $1 \mathrm{~m}$. W. of Stoke Pt.; not uncommon on coarse sand and medium gravel (R.A.T.): Rame-Eddystone Gds. (E.W.L H.).

Females in berry: Jan.; Mar.-Apr. (R.A T.): Dec. (E.w.L.H.). Hatching out: Apr. (R.A.T.). Zoæa stage : Apr.-May, (R.A.T., R.G.).

Cheraphilus nanus (Kröyer): T. Bell, Hist. Brit. Stalk-eyed Crust., p. 268, fig. (as Crangon bispinosus).

At the surface in the Sound, Feb. 1887 (w.H.).

Females in berry: June-July (R.A.T.). Zoæa stage: June; Aug.Sept. (R.G.).

Cheraphilus trispinosus (Hailstone): T. Bell, Hist. Brit. Stalk-eyed Crust., p. 265, fig. (as Crangon).

The Sound, occasionally; Yealm Est., occasionally; Whitsand B., common (R.A.T.): Cawsand B. (W.I.B.).

Females in berry: Apr. (T.v.H.): May-June (R.A.T.): July (w.G.); Aug. (J.T.C.). Hatching: May (R.A.T.). Zoæa stage: Apr-May: Sept. (R.G.).

\section{Nikidæ.}

Nika edulis, Risso: T. Bell, Hist. Brit. Stalk-eyed Crust., p. 275, fig.

The Sound, occasionally, but rare (w.G.): Cawsand B., sometimes moderately common; occasionally outside in $20-30$ fms. (R,A T.) : Jennycliff B. (A.J.S.).

Females in berry: Feb. (s.P., A.J.S.); May (W.G.): July (R.A.T.). Hatching: Apr. (R.A.T.). Zoæa stage: Apr.-June; Aug.-Sept. (R.G.). Late stages: Sept. (R.G.).

\section{Nephropsidæ.}

Homarus vulgaris, * Milne-Edwards: $T$. Bell, Hist. Brit. Stalk-eyed Crust., p. 242, fig.

Common among rocks, occasionally between tide-marks; Rum B.; Wembury B.; efe. (R.A.T.): occasionally on sand; Eddystone Gds. (E.J.A.).

Females in berry: May-June (R.A.T.) : July (w.G., R.A.T.): Aug. (w.G.): Sept.-Dec. (R.A.T.). Hatching: May-June (w.G.): July (R.A.T.). Larvæ, newly hatched and first moult, very abundant at the surface, July 1897 (w.I.B.): larvæ at the surface, Aug. 1897 (E.W.L.H.).

* If the "law of priority" is to be rigidly followed, this species must be termed Astacus gammarus (Linn.). 
[ Macrura-contd.]

\section{Scyllaridæ.}

Arctus ursus, Dana: C. Heller, Crust. südl. Europa, p. 195 (as Scyllarus arctus).

Millbay Dock, single spec., Apr. 1892 (R.A.T.): off the Breakwater Lt., single spec. in a crab-pot, Aug. 1897 (J.T.c.): $5 \mathrm{~m}$. S. of the Eddystone, single spec. Jan. 1900 (R.A.T.).

Breeding: Mar. (S.P.).

\section{Palinuridæ.}

Palinurus vulgaris, Latreille: T. Bell, Hist. Brit. Stalk-eyed Crust., p. 213, fig.

Common among rocks; the Sound; etc. (R.A.T.).

Females in berry: Apr.-May (R A.T.): Dec. (w.H.). Hatching: July (J.T.c.). Phyllosoma stage: Mar. (R.G.).

\section{Galatheidæ.}

Galathea dispersa, Spence Bate: J. Bonnier, Bull. Sci. France Belg., ser. 3 , vol. i, p. 154 , fig.

Abundant everywhere, 10-30 fms. (s.P.): Queen's Gd., common; Duke Rk., common; Cawsand B., occasionally (R.A.T.): Millbay Pit (w.I.B., R.A.T.) : Rame-Eddystone Gds.; Mewstone Gds. ; etc. (w.I.B., R.A.T., S.P.): Eddystone Gds., on all the grounds and in almost every haul (E.J.A.).

Breeding : Mar. (w.G., R.A.T.): May (s.P.).

Galathea intermedia, Lilljeborg: J. Bonnier, Bull. Sci. France Belg., ser. 3, vol. i, p. 130, fig.

Asia Sh.; Millbay Ch.; 3 m. S.S.W. Breakwater Lt.; 5 m. S.W. Penlee; 1 m. S.S.W. Yealm Hd. (W.I.B.): Queen's Gd.; Mewstone Gds.; Rame-Eddystone Gds. (R.A.T. \& w.I.B.).

Breeding: Mar. (w.G., R.A.T.).

Galathea squamifera, Leach: J. Bonnier, Bull. Sci. France Belg., ser. 3 , vol. i, p. 143 , fig.

Abundant under stones on all rocky shores, L.W.-3 fms. (S.P.): Millbay Ch; Queen's Gd.; Yealm R.; occasionally (R.A.T.).

Breeding: Jan.-May (R.A.T.).

Galathea strigosa (Linnæus): J. Bonnier, Bull. Sci. France Belg., ser. 3, vol. i, p. 160 , fig.

The Breakwater, occasionally; Mt. Edgcumbe (R.A.T., S.P.): Drake's I. (т.v.H.): off Batten Breakwater ${ }^{1}$; off Yealm Hd., 18 fms. ${ }^{1}$ (J.T.C.).

\section{Callianassidæ.}

Upogebia stellata (Montagu): T. Bell, Hist. Brit. Stalk-eyed Crust., p. 223, fig. (as Gebia).

Occasional specs. between tide-marks in the Yealm and under Mt. Edgeumbe, also in dredgings from Queen's Gd, and a single spec. $5 \mathrm{~m}$. W. $\frac{1}{4} \mathrm{~S}$. of Rame Hd. (R.A.T.).

Breeding: Aug. (R.A.T.).

\section{Thaumastochelidæ.}

Calocaris Macandrea: T. Bell, Hist. Brit. Stalk-eyed Crust., p. 233, fig. Zoæa stage: Aug.-Sept. (R.G.). 


\section{Paguridæ.}

Eupagurus Bernhardus (Linnæus): T. Bell, Hist. Brit. Stalk-eyed Crust., p. 171, fig. (as Pagurus).

Generally distributed on sand, gravel and stones, 3-30 fms. (R.A.T., S.P.).

The shell inhabited by this species usually affords lodgment for one or more specs. of Adamsia polypus [= Sagartia parasitica], or else it is invested with a colony of Hydractinia echinata; also its apical whorls are occupied by the Polychæte Nereis fucata (R.A.T., S.P.).

Females in berry: Feb. (R.A.T.): Apr. (w.G., R.A.T.). Zoæa stage: Mar. (R.G.).

Eupagurus cuanensis (Thompson): T. Bell, Hist. Brit. Stalk-eyed Crust., p. 178, fig. (as Pagurus).

Mewstone Gds., not uncommon, generally with Suberites; RameEddystone Gds., occasionally (R.A.T.): Eddystone Gds., never in large numbers, and confined to the gravel grounds in the neighbourhood of the Eddystone (E.J.A.): Yealm R., occasionally (R.A.T.).

Females in berry: Mar.-Apr. (R.A.T.).

Eupagurus Prideauxi (Leach): $T$. Bell, Hist. Brit. Stalk-eyed Crust., p. 175, fig. (as Pagurus).

Moderately common, 15-30 fms. (R.A.T.): Eddystone Gds., absent on the fine sand of the 'inner' and 'outer' trawling grounds (E.J.A.).

Almost always associated with Adamsia palliata (R.A.T., S.P.): a spec. trawled $2 \mathrm{~m}$. S.W. of Penlee had an Adamsia polypus upon its shell as well as the normal $A$. palliata (E.W.L.H): occasionally the shell is invested with a colony of Hydractinia echinata in place of the Adamsia palliata, and very rarely both are present upon the same shell (s.P.).

Females in berry: Jan. (R.A.T.) : Mar.-July (w.G., R.A.T.): Sept. (s.P.). Hatching out: Mar.-Apr. (R.A.T.) : June (s.P.): July-Aug. (R.A.T.). Zoæa stage: Mar.-Apr. (R.G.).

Eupagurus sculptimanus (Lueas): T. Bell, Hist. Brit. Stalk-eyed Crust., p. 186, fig. (as Pagurus Forbesi).

Rame-Eddystone Gds., in Suberites (R.A T.).

Diogenes varians, Costa: C. Heller, Crust. südl. Europa, p. 170, fig.

Whitsand B. (т.v.H.).

Breeding: July (w.G.).

Anapagurus læivis (Thompson): T. Bell, Hist. Brit. Stalk-eyed Crust., p. 184, fig. (as Pagurus).

Common on the Mewstone Gds. with Epizoanthus incrustatus; the Sound, occasionally; moderately common on other grounds, 15-30 fms. (R.A.T.): Eddystone Gds. (E J.A.).

Breeding: Feb. (R.A.T.): Apr. (w.G., R.A.T.): May (w.G.): July (R.A.T.): Aug. (S.P.). 


\section{BRACHYURA.}

\section{Porcellanidæ.}

Porcellana longicornis (Linnæus): T. Bell, Hist. Brit. Stalk-eyed Crust., p. 193, fig.

More or less abundant on all grounds, L.W.-30 fms. (S.P.).

Females in berry: Mar.-Apr. (W.G., R.A.T., S.P.): May (W.G., S.P.): June (w.G., R.A.T.): July (w.G., R.A.T., s.P.): Aug. (R.A.T.). Zoæa stage : Mar. (R.G.).

Porcellana platycheles (Pennant): T. Bell, Hist. Brit. Stalk-eyed Crust., p. 190, fig.

Under stones on all rocky shores, L.W.-3 fms. (s.P.).

Females in berry : Mar. (R.A.T.): Apr. (W.G., R A.T.): May (w.G.) : June-July (w.G., S.P.): Aug. (R.A.T.). Zoæa stage: Aug. (R.G.).

\section{Dromiidæ.}

Dromia vulgaris, Milne-Edwards: T. Bell, Hist. Brit. Stalk-eyed Crust., p. 369 , fig.

Not yet observed in the Plymouth district, although specs. are occasionally taken a little outside the district on the Cornish coast (s.P.).

\section{Leucosiidæ.}

Ebalia Cranchi, Leach: T. Bell, Hist. Brit. Stalk - eyed Crust., p. 148, fig.

Cawsand B. ${ }^{1}$; Whitsand B. ${ }^{1}$ (T.V.H.): Eddystone Gds., on sandy gravel, E. of Hand Deeps (E.J.A.).

Ebalia tuberosa (Pennant): T. Bell, Hist. Brit. Stalk-eyed Crust., p. 141, fig. (as E. Pennanti).

Millbay Ch. (т.v.H.): not uncommon, 20-30 fms.; Mewstone Gds.; Rame-Eddystone Gds. ; etc. (R.A.T.): Eddystone Gds., together with $E$. tumefacta (E.J.A.).

Females in berry : May-June (R.A.T.).

Ebalia tumefacta (Montagu): T. Bell, Hist. Brit. Stalk-eyed Crust., p. 145 , fig. (as E. Bryeri).

Duke Rk., single spec. (т.V.H.): Eddystone Gds., abundant on coarse sandy gravel W. of the Eddystone, constantly present but less abundant on clean shell-gravel grounds (E.J.A.).

\section{Inachidæ.}

Macropodia egyptia: A. Milne-Edwards, Hist. Nat. Crust., vol. i, p. 280 .

Not uncommon on weedy ground (w.G.): very rare, 15-30 fms. (R.A.T.).

Macropodia longirostris (Fabricius): T. Bell, Hist. Brit. Stalk-eyed Crust., p. 6, fig. (as Stenorynchus tenuirostris).

Queen's Gd., not uncommon; Cawsand B., occasionally; moderately common outside the Sound, 15-30 fms.; Mewstone Gds.; Rame-Eddystone Gds.; etc. (R.A.T.): Eddystone Gds., especially abundant where the prevailing Hydroid is Sertularella Gayi (E.J.A.): Yealm R., occasionally (R.A.T.).

Breeding: Feb.-Apr. (R.A.T.) : May (w.G.) : July-Aug. (R.A.T.). 
[Brachyura-contd.]

Macropodia rostratus (Linnæus) : T. Bell, Hist. Brit. Stalk-eyed Crust.,

p. 2, fig. (as Stenorynchus phalangium).

Occasionally in dredgings from the Sound; Cawsand B., moderately common; Yealm R., not uncommon (R.A.T.).

Breeding: May (w.G.): Aug. (R.A.T.).

Acheus Cranchi, Leach: T. Bell, Hist. Brit. Stalk-eyed Crust., p. 10, fig. Queen's Gd.; Duke Rk. (T.V.H.): Yealm R. (R.A.T.).

Breeding: Aug. (R.A.T.) : Sept. (S.P.).

Inachus Dorsettensis (Pennant): T. Bell, Hist. Brit. Stalk-eyed Crust., p. 13, fig.

Queen's Gd., occasionally; common in $15-30 \mathrm{fms}$. outside the Sound; Mewstone Gds.; Rame-Eddystone Gds.; etc. (R.A.T.): Eddystone Gds. (E.J.A.): Yealm R., not uncommon (R.A.T.).

Females in berry: Jan.-Apr. (R.A.T.): June-Aug. (R.A.T., s.P.) : Sept. (s.P.). Zoæa stage: Mar.; July (R.G.).

Inachus Dorynchus, Leach: T. Bell, Hist. Brit. Stalk-eyed Crust., p. 16, fig.

Millbay Ch., occasionally; Duke Rk.; Yealm R., not uncommon (R.A.T.).

Female in berry: Mar. (R.A.T.).

\section{Maiidæ.}

Maia squinado (Herbst): T. Bell, Hist. Brit. Stalk-eyed Crust., p. 39, fig.

Moderately common in the Sound, among rocks and on sand with Zostera, etc., 5 fms. (R.A.T.): common amongst the rocks outside Plymouth Sound (E.J.A.): Cawsand B., not uncommon (R.A.T., S.P.): Mewstone 'Echinoderm' Gd.; Rame-Eddystone Gds.; occasionally (R.A.T.): Eddystone Gds.(E.J.A.): Yealm R., moderately common(R.A.T.).

Breeding: Mar.-Apr. (R.A.T.): May (s.P.): June (R.A.T.).

Pisa biaculeata (Montagu): T. Bell, Hist. Brit. Stalk-eyed Crust., p. 27, fig. (as P. Gibbsi).

Mewstone Ledge, single spec.; Yealm R., single spec. (R.A.T.).

Breeding: May (R.A.T.).

Hyas araneus (Linnæus): T. Bell, Hist. Brit. Stalk-eyed Crust., p. 31, fig.

Yealm R. (E.J.A., E.W.L.H., R.A.T., T.V.H.): the Mewstone (T.v.H.).

Hyas coarctatus, Leach: $T$. Bell, Hist. Brit. Stalk-eyed Crust., p. 35, fig.

Occasionally in 15-30 fms.; Rame-Eddystone Gds.; etc. (R.A.T.):

Eddystone Gds., occasionally on the fine sand grounds (E.J.A.):

Cawsand B., fairly common (A.J.S.).

Breeding: Mar.-Apr.; July (R.A.T.): Aug. (w.G.): Dec. (R.A.T.).

\section{Parthenopidæ.}

Eurynome Aspera (Pennant): T. Bell, Hist. Brit. Stalk-eyed Crust., p. 46, fig.

Fairly common, 10-30 fms., on gravel and sand (s.P.): Millbay Ch. and Pit, not uncommon; Asia Sh.; Queen's Gd. (R.A.T., s.P.): Duke Rk.; the Breakwater, between tide-marks ${ }^{1}$; Yealm R., occasionally in dredgings (R.A.T.): Rame-Eddystone Gds.; Mewstone 'Amphioxus' Gd. (R.A.T., S.P.): Eddystone Gds. (E.J.A., S.P.): Stoke Pt. Gds. (S.P.).

Breeding: Jan.-Feb. (w.G., R.A.T.): Mar.-Apr. (W.G., R.A.T., S.P.) May-June (s.P.) : July (R.A.T., s.P.): Sept. (s.P.). 
[Brachyura-contd.]

\section{Cancridæ.}

Cancer pagurus, Linnæus: T. Bell, Hist. Brit. Stalk-eyed Crust., p. 59, fig.

Small specs. common among stones between tide-marks on all rocky shores; large specs. seldom seen above low-water mark (s.P.).

Breeding: Jan. (R.A.T.): Feb., onwards (w.G.): Mar.-July; Dec. (R.A.T.).

Pirimela denticulata (Montagu): T. Bell, Hist. Brit. Stalk-eyed Crust., p. 72 , fig.

Drake's I., not uncommon on clean coarse shelly gravel (w.G., s.P.): Rum B. (R.A.T.): Yealm R. (т.v.H.): Stoke Pt. Gds. (s.P.).

Breeding: Feb.; Apr. (R A.T.).

Xantho hydrophilus (Herbst): $T$. Bell, Hist. Brit. Stalk-eyed Crust., p. 54 , fig. (as $X$. rivulosa).

Between tide-marks on all rocky shores, not uncommon under stones, etc. (S.P.).

Breeding: Mar. (R.A.T.): May (w.G., s.P.): June (w.G.).

Xantho incisus, Leach: T. Bell, Hist. Brit. Stalk-eyed Crust., p. 51, fig. (as $X$. florida).

Common between tide-marks on all rocky shores (s.P.).

Breeding: Apr. (R.A.T.): May (w.G.): June (w.G., R.A.T.).

\section{Eriphidæ.}

Pilumnus hirtellus (Linnæus): T. Bell, Hist. Brit. Stalk-eyed Crust, p. 68 , fig.

Not uncommon on all rocky shores under stones and in crevices, L.W.-5 fms. (s.P.): common in Lepralia foliacea; Mewstone Ledge ; etc. (R.A.T., S.P.).

Breeding: Mar. (s.P.): Apr. (w.G., R.A.T.): May-June (w.G.): July (S.P.).

\section{Portunidæ.}

Portunus arcuatus, Leach: T. Bell, Hist. Brit. Stalk-eyed Crust., p. 97, fig.

Queen's Gd. (R.A.T.): Cawsand B. (т.V.H., E.w.L.H.): Yealm R., not uncommon (R.A.T., T.V.H.): Laira (T.V.H.).

Breeding: Mar. (w.G.): Apr.-May (w.G., R.A.T.).

Portunus corrugatus (Pennant): T. Bell, Hist. Brit. Stalk-eyed Crust., p. 94 , fig.

Queen's Gd.; Millbay Ch.; not uncommon (R.A.T.): Drake's I., between tide-marks (T.v.H. $\left.{ }^{1}\right)$ : Cawsand B. (E.w.L.H.): Bovisand B. (J.T.C.).

Breeding: June; Aug. (R.A.T.).

Portunus Depurator (Linnæus): T. Bell, Hist. Brit. Stalk-eyed Crust., p. 101, fig.

Generally distributed, 3-30 fms., sometimes in large numbers (R.A.T., S.P.).

Breeding: Jan.-Feb. (R.A.T.): Mar.-Apr. (W.G., R.A.T., S.P.): MayJuly (w.G., R.A.T.) : Aug. (w.G.): Sept.; Nov. (R.A.T.). 
[Brachyura-contd.]

Portunus holsatus, Fabricius: T. Bell, Hist. Brit. Stalk-eyed Crust., p. 109, fig.

Queen's Gd.; Cawsand B.; Whitsand B. (E.w.L.H.).

Breeding: Mar. (w.G.).

Portunus marmoreus, Leach: T. Bell, Hist. Brit. Stalk-eyed Crust., p. 105, fig.

Breeding: May (w.G.).

Portunus puber (Linnæus): T. Bell, Hist. Brit. Stalk-eyed Crust., p. 90 , fig.

Common among stones on all rocky shores, between tide-marks5 fms. (s.P.).

Females in berry: Feb.-May; July (R.A.T.). Zoæas hatching out: Mar.-May (R.A.T.).

Portunus pusillus, Leach: T. Bell, Hist. Brit. Stalk-eyed Crust., p. 112, fig.

Not uncommon in dredgings from the Sound and outside grounds, 15-30 fms.; Queen's Gd., occasionally; Drake's I., occasionally between tide-marks; Yealm R., not uncommon (R.A.T.): Eddystone Gds., on gravel, and occasionally on sand grounds adjoining the gravel (E.J.A.).

Females in berry: Feb.-May (R.A.t.). Hatching out: Mar.; June (R.A.T.).

Bathynectes longipes (Risso): T. Bell, Hist. Brit. Stalk-eyed Crust., p. 361, fig. (as Portunus).

Near the Eddystone, on fine sand (w.G.).

Carcinus menas (Pennant): T. Bell, Hist. Brit. Stalk-eyed Crust., p. 76, fig.

Common everywhere, tide-marks-3 fms. (R.A.T., S.P.): Millbay Ch., occasionally (R.A.T.).

Females in berry: Jan.-Apr. (w.G., R.A.T.): May-Aug. (w.G.) : Nov. (R.A.t.): Dec. (w.G., R.A.T.). Zoæa stage: Feb.-Apr. (R.G.): July (R.A.T.).

Portumnus bigutratus (Risso): C. Heller, Crust. südl. Europa, p. 94 (as Platyonichus nasutus).

Drake's I., occasional specs. burrowing in fine gravel (w.G., R.A.T.).

Females in berry: Aug. (R.A.T.). Megalops stage: Aug. (R.G.).

Polybius Henslowi, Leach: T. Bell, Hist. Brit. Stalk-eyed Crust., p. 116, fig.

The $t$ is not uncommon at times on the shrimp-trawling grounds, particularly in Cawsand B., also swimming near the surface at the deeper water stations, but the $q$ has only twice been recorded at the Laboratory (S.P.).

Females in berry: Sept. (R.A.T., W.I.B.). Hatched: Sept. (R.A.T.).

Corystidæ.

Atelecyclus septemdentatus (Montagu): T. Bell, Hist. Brit. Stalkeyed Crust., p. 153, fig. (as A. heterodon).

Moderately common 15-30 fms. (R.A.T.): local but common where it occurs, generally on muddy gravel (s.P.): Rame-Eddy- 
[ Brachyura-contd. : Pycnogonida ]

stone Gds.; Mewstone Gds. (R.A.T., S.P.): Eddystone Gds., abundant on muddy gravel about $1 \mathrm{~m}$. E. of the Eddystone, on gravelly sand about $3 \mathrm{~m}$. W. of the Eddystone, and occasionally at other stations (E.J.A.).

Breeding: Apr.-July (R.A.T.).

Corystes Cassivelaunus (Pennant): T. Bell, Hist. Brit. Stalk-eyed Crust., p. 159 , fig.

Generally distributed on clean fine sand, 15-30 fms.; Cawsand B., not uncommon (R.A.T., S.P.).

Females in berry: May-July; Nov. (R.A.T.). Zoæa stage: Feb.May (R.G.). Megalops stage: Apr. (R.G.).

\section{Pinnotheridæ.}

Pinnotheres Pisum (Linnæus): T. Bell, Hist. Brit. Stalk-eyed Crust., p. 121, fig.

Mewstone 'Amphioxus' Gd.', in the mantle-cavity of Cardium norvegicum and of Glycimeris (W.G. \& R.A.T.).

Breeding: July-Aug. (R.A.T.).

\section{Ocypodidæ.}

Gonoplax Rhomboides (Linnæus): T. Bell, Hist. Brit. Stalk-eyed Crust., p. 130 , fig. (as $G$. angulata).

Cawsand B., rare; Jennycliff B. ${ }^{1}$ (s.P.): occasionally in $15-30$ fms.; Rame-Eddystone Gds.; Mewstone Gds. (R.A.T., s.P.): Eddystone Gds. (E.J.A., ${ }^{1}$ S.P.).

Hatched out in June (R.A.T.).

\section{PYCNOGONIDA.}

Pycnogonidæ.

Pycnogonum littorale (Strœm): G. O. Sars, Zool, Norske NordhavsExped., Pycnog., p. 7, fig.

Yealm R.; Breakwater (T.v.H.).

\section{Phoxichilidæ.}

Phoxichilus spinosus (Montagu): P. P. C. Hoek, Arch. Zool. expt, vol. ix, p. 518, fig.

Queen's Gd., occasionally (R.A.T.): Millbay Pit, several specs.; Yealm R. (T.v.H.).

\section{Phoxichilidiidæ.}

Anaplodactylus petiolatus (Kröyer): G. O. Sars, Zool. Norske

Nordhavs-Exped., Pyenog., p. 25, fig.

Asia Sh. (A.M.N., S.P.).

Pallenidæ.

Pallene Brevirostris, Johnston: P. P. C. Hoek, Arch. Zool, expt., vol. ix, p. 511, fig.

Asia Sh. (S.P.).

\section{Nymphonidæ.}

Nymphon gallicum: P. P. C. Hoek, Arch. Zool, expt., vol. ix, p. 501, fig. Occasionally between tide-marks in the Sound and in $2-5 \mathrm{fms}$. in Cawsand B. (R.A.T.).

Males carrying ova in Feb. (R.A.T.). 
[Pycnogonida—contd:: Bryozoa ]

Nymphon Gracile, Leach: P. P. C. Hoek, Arch. Zool. expt., vol. ix, p. 498 , fig.

Duke Rk., rare; Yealm R. (т.v.H.).

A male was observed carrying ova in May (R.A.T.).

Nymphon rubrum, Hodge: G. O. Sars, Zool. Norske Nordhavs-Exped., Pycnog., p. 58, fig.

Asia Sh. (A.M.N. $\left.{ }^{1}\right)$.

\section{Ammotheidæ.}

Ammothea echinata (Hodge): P. P. C. Hoek, Arch. Zool. expt., vol. ix, p. 508, fig.

Not uncommon, Millbay Ch. and Pit, Asia Sh., Queen's Gd., Yealm R., Rum B., Duke Rk., etc. (R.A.T.).

Breeding, Aug. (w.G.).

\section{BRYOZOA.}

\section{Aeteidæ.}

Aetea anguina (Linnæus): T. Hincks, Hist. Brit. Mar. Polyzoa, p. 4, fig. Queen's Gd., occasionally on Algæ; common on rocky ground

S. of the Breakwater (R.A.T.).

Aetea Recta, Hincks: T. Hincks, Hist. Brit. Mar. Polyzoa, p. 6, fig.

Not uncommon on shells, etc., 20-35 fms.; Mewstone Gds.; Rame-Eddystone Gds. (R.A.T.).

\section{Eucrateidæ.}

Eucratea chelata (Linnæus): T. Hincks, Hist. Brit. Mar. Polyzoa, p. 14, fig.

On a hulk near the Breakwater (R.A.T.): between Rame Hd. and the Eddystone, on Bowerbankia (E.J.A.).

\section{Cellulariidæ.}

Scrupocellaria reptans (Linnæus): T. Hincks, Hist. Brit. Mar. Polyzoa, p. 52, fig.

Plymouth (S.F.H.).

Scrupocellaria scrupea, Busk: T. Hincks, Hist. Brit. Mar. Polyzoa, p. 50, fig.

Mewstone, 25 fms. (s.F.H.).

Scrupocellaria scruposa (Linnæus): T. Hincks, Hist. Brit. Mar. Polyzoa, p. 45 , fig.

Common S. of the Breakwater, 20-35 fms.; Mewstone Gds.; Rame-Eddystone Gds.; Stoke Pt. Gds. (R.A.T., s.P.): Eddystone Gds., plentiful on the fine sand and gravels, generally attached to Polychrte tubes or to Cellaria, rare on the shell-gravel grounds (E.J.A.).

Caberea Boryi (Audouin): T. Hincks, Hist. Brit. Mar. Polyzoa, p. 61, fig.

Plymouth (S.F.H.). 


\section{Bicellariidæ.}

[Bryozoa-contd. $]$

Bicellaria ciliata (Linnæus): T. Hincks, Hist. Brit. Mar. Polyzoa, p. 68 , fig.

Moderately common, 5-35 fms., on Cellaria and on Hydroids (R.A.T.): Millbay Pit; Asia Sh. (R.A.T., S.P.): Queen's Gd., not uncommon (s.P.): Duke Rk. (E.J.A.): Mewstone Ledge, occasionally (R.A.T.): Rame-Eddystone Gds. (R.A.T., S.P.): Eddystone Gds., not uncommon on various Hydroids (E.J.A., S.P.).

With ovicells: March (R.A.T.).

Bugula avicularia (Linnæus): T. Hincks, Hist. Brit. Mar. Polyzoa, p. 75 , fig.

Eddystone Gds. (E.J.A, S.P.); Rame-Eddystone Gds. (s.P.).

Bugula flabellata, J. E. Gray: T. Hincks, Hist. Brit. Mar. Polyzoa, p. 80 , fig.

Common in dredgings from the Sound (R.A.T., S.P.): Mewstone

Ledge, moderately conmon (R.A.T.): Eddystone Gds., occasionally (E.J.A.).

Breeding: July, Sept. (R.A.T.).

Bugula plumosa (Pallas): T'. Hincks, Hist. Brit. Mar. Polyzoa, p. 84, fig. Plymouth, common (s.F.H.).

Bugula turbinata, Alder: T. Hincks, Hist. Brit. Mar. Polyzoa, p. 77, fig. Moderately common in dredgings from the Sound (R.A.T., S.P.).

\section{Flustridæ.}

Flustra papyracea, Ellis \& Solander: T. Hincks, Hist. Brit. Mar. Polyzoa, p. 118, tig.

Millbay Ch., occasionally (s.P.): Queen's Gd. (R.M.P.).

Flustra (? sp.) securifrons (Pallas): T. Hincks, Hist. Brit. Mar. Polyzoa, p. 120 , fig. Millbay Ch., occasionally (R.A.T.).

\section{Membraniporidæ.}

Membranipora aurita, Hincks: T. Hincks, Hist. Brit. Mar. Polyzoa, p. 159 , fig. Mewstone, 20 fnus. (T.H.T. $\left.{ }^{1}\right)$.

Membranipora catenularia (Jameson): T. Hincks, Hist. Brit. Mar. Polyzoa, p. 134, fig.

Mewstone Gds. (R.A.T.): Eddystone Gds. (E.J.A.).

Membranipora curvirostris, Hincks: T. Hincks, Hist. Brit. Mar. Polyzoa, p. 153, fig. Eddystone Gds. (R.M.P.).

Membranipora Dumerili (Audouin): T. Hincks, Hist. Brit. Mar.

Polyzoa, p. 156, fig. Eddystone Gds. (E.J.A.).

Membranipora Flemingi, Busk: T'. Hincks, Hist. Brit. Mar. Polyzoa, p. 156, fig. Eddystone Gds. (E.J.A.).

Membranipora flustroides, Hincks: T. Hincks, Hist. Brit. Mar. Polyzoa, p. 151, fig. Mewstone, 25 fms. (s.F.H.). 
[Bryozoa-contd.]

Membranipora imbellis, Hincks: T. Hincks, Hist. Brit. Mar. Polyzoa, p. 160 , fig.

Plymouth, a doubtful fragment (s.F.H.).

Membranipora Lacroixi, Audouin: T. Hincks, Hist. Brit. Mar. Polyzoa, p. 129 , fig.

Plymouth (R.M.P.).

Membranipora lineata (Linnæus): T. Hincks, Hist. Brit. Mar. Polyzoa, p. 143 , fig.

Cawsand B. (т.н.т.).

Membranipora membranacea (Linnæus): T. Hincks, Hist. Brit. Mar. Polyzoa, p. 140, fig

Generally distributed, on Laminaria, etc. (R.A.T., S.P.).

Membranipora pilosa (Linnæus): T. Hincks, Hist. Brit. Mar. Polyzoa, p. 137 , fig.

Common everywhere on Fucus, etc., between tide-marks (R.A.T., S.P.).

Membranipora Rosseli (Audouin): T'. Hincks, Hist. Brit. Mar. Polyzoa, p. 166, fig.

Queen's Gd.; Cawsand B.; Mewstone, 20 fms. (s.F.H.).

\section{Microporidæ.}

Micropora coriacea (Esper): T. Hincks, Hist. Brit. Mar. Polyzoa, p. 174, fig.

Mewstone, 25 fms. (s.F.H.).

\section{Cellariidæ.}

Cellaria fistulosa (Linnæus): T. Hincks, Hist. Brit. Mar. Polyzoa, p. 106, fig.

Common with C. sinuosa, especially on fine sand grounds, 15-30 fms. (E.J.A.).

Cellaria salicornioides, Lamouroux: T. Hincks, Hist. Brit. Mar. Polyzoa, p. 112, fig. (as C. Johnsoni).

Eddystone Gds. (E.J.A.).

Cellaria sinuosa (Hassall): T. Hincks, Hist. Brit. Mar. Polyzoa, p. 109 , fig.

Common with $C$. fistulosa, especially on fine sand grounds $15-30$ fms. (E.J.A.).

\section{Tubicellariidæ.}

Lagenipora socialis, Hincks: T. Hincks, Hist. Brit. Mar. Polyzoa, p. 235 , fig.

Mewstone, 25 fms. (s.F.H.).

\section{Cribrilinidæ.}

Cribrilina figularis (Johnston): T. Hincks, Hist. Brit. Mar. Polyzoa, p. 196, fig.

Mewstone 'Echinoderm ’ Gd. (R.A.T.); Eddystone Gds. (E.J.A.).

With ovicells: Apr. (R.A.T.).

Cribrilina punctata (Hassall): T. Hincks, Hist. Brit. Mar. Polyzoa, p. 190 , fig.

Mewstone, 25 fms. (s.F.H.). 
[Bryozoa-contd.]

Cribrilina radiata (Moll): T. Hincks, Hist. Brit. Mar. Polyzoa, p. 185, fig. Mewstone Ledge, very common (s.F.H.): Rame-Eddystone Gds., on Pecten maximus (R.A.T.): Eddystone Gds. (E.J.A.).

Membraniporella nitida (Johnston): T. Hincks, Hist. Brit. Mar.

Polyzoa, p. 200, fig.

Common on Lepralia foliacea (s.F.H.): Mewstone, 20 fms. (т.н.т.).

\section{Microporellidæ.}

Microporella ciliata (Pallas): T. Hincks, Hist. Brit. Mar. Polyzoa, p. 206, fig.

Mewstone, 20 fms., var. personata (s.F.H.): Rame-Eddystone Gds., occasionally, on Pecten maximus (R.A.T.) : Eddystone Gds. (E.J.A.).

Microporella impressa (Audouin): T. Hincks, Hist. Brit. Mar. Polyzoa, p. 214, fig.

Common on red sea-weeds (S.F.H.).

Microporella Malusir (Audouin): T. Hincks, Hist. Brit. Mar. Polyzoa, p. 211, fig.

Mewstone Ledge, very common (s.F.H.): Eddystone Gds. (E.J.A.).

Microporella violacea (Johnston): T. Hincks, Hist. Brit. Mar. Polyzoa, p. 216, fig.

Mewstone Ledge (s.F.H.): Eddystone Gds. (E.J.A.).

\section{Escharidæ.}

Lepralia foliacea (Ellis \& Solander): T. Hincks, Hist. Brit. Mar. Polyzoa, p. 300, fig.

Millbay Pit, occasionally; Queen's Gd., not uncommon; Mewstone Ledge, abundant; more or less common on all the outside grounds, 10-30 fms. (s.P.).

Lepralia Pallasiana (Moll): T. Hincks, Hist. Brit. Mar. Polyzoa, p. 297, fig.

Yealm R., $1 \frac{1}{2}$ fms. (т.H.T. $\left.{ }^{1}\right)$ : Yealm Sand-bank, between tidemarks (R.M.P.).

Lepralia pertusa (Esper): T. Hincks, Hist. Brit. Mar. Polyzoa, p. 305 , fig.

Yealm R. ${ }^{1}$; Eddystone Gds. (E.J.A.).

Umbonula verrucosa (Esper): T. Hincks, Hist. Brit. Mar. Polyzoa, p. 317, fig.

Drake's I., on rocks, between tide-marks (R.A.T., E.J.A.): Wembury B. (E.J.A.).

Chorizopora Brongniarti (Audouin): T. Hincks, Hist. Brit. Mar. Polyzoa, p. 224, fig.

Rame-Eddystone Gds., on Pecten maximus (R.A.T.): Eddystone Gds. (E.J.A.).

Porella concinna (Busk): T. Hincks, Hist. Brit. Mar. Polyzoa, p. 323, fig.

Mewstone, 20 fms. (s.F.H. $\left.{ }^{1}\right)$ : Eddystone Gds. (E.J.A.).

Escharoides quincuncialis (Norman): T. Hincks, Hist. Brit. Mar.

Polyzoa, p. 339, fig.

Plymouth, single spec., Apr. 1889 (s.r.H.). 
[Bryozoa-contd.]

Smittia cheilostoma (Manzoni): T. Hincks, Hist. Brit. Mar. Polyzoa, p. 349 , fig.

Eddystone Gds. (E.J.A.).

Smittia Landsborovil (Johnston): T. Hincks, Hist. Brit. Mar. Polyzoa, p. 341, fig.

Mewstone Ledge (s.F.H.).

Smittia trispinosa (Johnston): T. Hincks, Hist. Brit. Mar. Polyzoa, p. 353, fig.

Mewstone Ledge, excessively common (s.F.H.): Eddystone Gds. (E.J.A.).

Phylactella collaris (Norman): T. Hincks, Hist. Brit. Mar. Polyzoa, p. 358, fig.

Eddystone Gds. (E.J.A.).

Mucronella coccinea (Abildgaard): T. Hincks, Hist. Brit. Mar. Polyzoa, p. 371, fig.

Plymouth, very common (s.F.H.): Eddystone Gds. (E.J.A.).

Mucronella Peachi (Johnston): T. Hincks, Hist. Brit. Mar. Polyzoa, p. 360 , fig.

Eddystone Gds. (E.J.A.).

Mucronella variolosa (Johnston): T. Hincks, Hist. Brit. Mar. Polyzoa, p. 366 , fig.

Eddystone Gds (E.J.A.).

Mucronella ventricosa (Hassall): T. Hincks, Hist. Brit. Mar. Polyzoa, p. 363, fig.

Mewstone 'Echinoderm' Gd.; Rame-Eddystone Gds. (R.A.T.):

Eddystone Gds. (E.J.A.).

Palmicellaria Skenei (Ellis \& Solander): Tr. Ifincks, Hist. Brit. Mar.

Polyzoa, p. 379, fig.

Eddystone Gds. (E.J.A.).

Rhynchozoon Bispinosum (Johnston): T. Hincks, Hist. Brit. Mar.

Polyzoa, p. 385, fig. (as Rhynchopora).

Eddystone Gds. (E.J.A.).

Hippothoa distans, Macgillivray: T. Hincks, Hist. Brit. Mar. Polyzoa, p. 293, fig. (as H. flagellum). Mewstone, 25 fms. (s.F.H.).

Hippothoa divaricata, Lamouroux: T. Hincks, Hist. Brit. Mar. Polyzoa, p. 288, fig.

Queen's Gds., 4 fms. (т.H.T. $\left.{ }^{1}\right)$ : Mewstone 'Echinoderm' Gd.; Rame-Eddystone Gds., on Pecten maximus (R.A.T.): Eddystone Gds. (E.J.A.).

Schizoporella armata (Hincks): T. Hincks, Hist. Brit. Mar. Polyzoa, p. 258 , fig.

Mewstone, 25 fms. (s.F.H. $\left.{ }^{1}\right)$.

Schizoporella auriculata (Hassall): T. Hincks, Hist. Brit. Mar.

Polyzoa, p. 260, fig.

Eddystone Gds. (E.J.A.). 
Schizoporella Cecilin (Audouin): T. Hincks, Hist. Brit. Mar. Polyzoa, p. 269, fig.

Eddystone, 20 fms. (s.F.H.).

Schizoporella cristata, Hincks: T. Hincles, Hist. Brit. Mar. Polyzoa, p. 254, fig.

Mewstone Ledge, two small colonies in dead Pecten shell (s.F.H.). Schizoporella hyalina (Linnæus): T. Hincks, Hist. Brit. Mar. Polyzoa, p. 271, fig.

Millbay Ch. (т.н.т.): Plymouth, common; Yealm Sand-bank, abundant on Algæ (R.M.P.).

Schizoponella Johnstoni, Quelch; T. Hincks, Hist. Brit. Mar. Polyzoa, p. 246 , fig. (as $S$. simplex).

Mewstone, 25 fms. (s.F.H.).

Schizoporella linearis (Hassall): T. Hineks, Hist. Brit. Mar. Polyzoa, p. 247 , fig.

Plymouth, very common (S.F.H.): Millbay Ch. (R.A.T.): Eddystone Gds. (E.J.A.).

Schizoporella spinifera (Johnston): T. Hincks, Hist. Brit. Mar. Polyzoa, p. 241, fig.

Plymouth (s.F.H.).

Schizoporella unicornis (Johnston): T. Hincks, Hist. Brit. Mar. Polyzoa, p. 238, fig.

Mewstone, 25 fms. (s.F.H.).

Schizotheca fissa (Busk): T'. Hincks, Hist. Brit. Mar. Polyzoa, p. 284, fig. Eddystone Gd. (E.J.A.).

\section{Celleporidæ.}

Cellepora avicularis, Hincks: T. Hincks, Hist. Brit. Mar. Polyzoa, p. 406 , fig.

Mewstone, 20 fms. (т.H.T.): Eddystone Gds. (E.J.A.).

Cellepora Costazin, Audouin: T'. Hincles, Hist. Brit. Mar. Polyzoa, p. 411, fig.

Cawsand B. (R.M.P.).

Cellepora dichotoma, Hincks: T. Hincks, Hist. Brit. Mar. Polyzoa, p. 403 , fig.

Eddystone, 20-30 fms. (S.F.H.).

Cellepora pumicosa, Linnæus: T. Hincks, Hist. Brit. Mar. Polyzoa, p. 399, fig.

Common at most outside stations (R.M.P.).

Cellepora ramulosa, Linnæus: T. Hincles, Hist. Brit. Mar. Polyzoa, p. 401, fig.

Eddystone Gds. (E.J.A., S.P.): Rame-Eddystone Gds. (R.A.T., S.P.) : Stoke Pt. Gds. (s.P.).

\section{Crisiidæ.}

Crisia aculeata, Hassall : S. F. Harmer, Quart. Journ. Micr. Sci., ser. 2, vol. xxxii., p. 132, fig.

Not uncommon, 4-5 fms., on red seaweeds, stones, and sponges (S.F.H., R.A.T.).

Ovicells: Apr., May (s.F.H., R.A.T.). 
[Bryozoa-contd.]

Crisia connuta, (Linnæus) : T. Hincks, Hist. Brit. Mar. Polyzoa, p. 419, fig. [excl., var. geniculata, $=C$. geniculata] .

Fairly common, mostly on red seaweeds (s.F.H.): Queen's Gd.; Mewstone Ledge ; Rame-Eddystone Gds. (R.A.T.).

Ovicells: commonest Apr.-May (s.F.H.).

Crisia denticulata (Lamarck): S. F. Harmer, Quart. Journ. Micr. Sci., ser. 2, vol. xxxii., p. 129 , fig.

Plymouth, seldom found (s.F.H.): Queen's Gd., not uncommon; Duke Rk., occasionally common (R.A.T.).

Crisia eburnea (Linnæus): S. F. Harmer, Quart. Journ. Mier. Sci., ser. 2, vol. xxxii,, p. 131, fig.

Eddystone Gds. (E.J.A., R.A.T.) : common, almost always on red weeds or Sertularia (S.F.H.).

Ovicells: Feb.-May (s.F.H., R.A.T.); commonest, Mar.-Apr. (s.F.H.).

Crisia geniculata: H. Milne Edwards, Ann. Sci. Nat., Zool, ser. 2, vol. ix, p. 197, fig.

Rare (R.A.T.).

Crisia ramosa : S. F. Harmer, Quart. Journ. Mier. Sci., ser. 2, vol. xxxii., p. 134, fig.

Plymouth, 4-30 fms., the commonest species; generally on stones, but also on shells, red seaweeds, Cellaria, sponges, etc.; grows most luxuriantly in 4-6 fms. (s.F.H.): Queen's Gd., occasionally (R.A.T.): Eddystone Gds. (E.J.A.).

Breeding: Feb. (R.A.T.): Apr.-Aug., maximum in May-June (s.F.H.).

\section{Diastoporidæ.}

Diastopora obelia, Johnston: T. Hincks, Hist. Brit. Mar. Polyzoa, p. 462 , fig.

Eddystone Gds. (E.J.A.).

Diastopora patina (Lamarck): T. Hincks, Hist. Brit. Mar. Polyzoa, p. 458 , fig.

Common on shells, etc., 15-35 fms. (R.A.T.): Mewstone Ledge, fairly common (s.F.H.): Rame-Eddystone Gds. (R.A.T.): Eddystone Gds. (E.J.A.).

Diastopora sarniensis, Norman: T. Hincks, Hist. Brit. Mar. Polyzoa, p. 463 , fig.

Common on shells, stones, etc., $15-35$ fms. (R.A.T.): RameEddystone Gds. (R.A.T.): Eddystone Gds. (E.J.A.).

Diastopora suborbicularis: T. Hincks, Hist. Brit. Mar. Polyzoa, p. 464, fig.

Mewstone Ledge (s.F.H.): Eddystone Gds. (E.J.A.).

\section{Tubuliporidæ.}

Tubulipora liliacea (Pallas): S. F. Harmer, Quart. Journ. Micr. Sci., ser. 2, vol. xli, p. 90, fig.

Duke Rk., very occasionally; not uncommon on shells, stones, Hydroids, etc., S. of the Breakwater, 15-30 fms.; Rame-Eddystone Gds (R.A.T.): Eddystone Gds. (E.J.A.). 
Tubulipora Phalangea, Couch: S. F. Harmer, Quart. Journ. Micr. Sci., ser. 2, vol. xli, p. 94, fig.

Plymouth, common, 3-15 fms. (s.F.H.).

Tubulipora plumosa, W. Thompson: S. F. Harmer, Quart. Journ. Micr.

Sci., ser. 2, vol. xli, p. 105, fig.

Plymouth district, abundant on Cystoseira granulata and on Saccorhiza bulbosa (S.F.H.).

Stomatopora granulata, Milne-Edwards: T. Hincks, Hist. Brit. Mar.

Polyzoa, p. 425, fig.

Eddystone Gds. (E.J.A.).

Stomatopora Johnstoni, Heller: T. Hincks, Hist. Brit. Mar. Polyzoa, p. 430 , fig.

Eddystone Gds. (E.J.A.).

Stematopora major, Johnston: T. Hincks, Hist, Brit. Mar. Polyzoa, p. 427 , fig.

Eddystone Gds. (E.J.A.).

\section{Lichenoporidæ.}

Lichenopora hispida (Fleming): T. Hincks, Hist. Brit. Mar. Polyzoa, p. 473 , fig.

Common on all grounds $15-30 \mathrm{fms}$. (E.J.A.).

\section{Alcyonidiidæ.}

Alcyonidium gelatinosum (Linnæus): T. Hincks, Hist. Brit. Mar. Polyzoa, p. 491, fig.

In dredgings from the Sound, Mewstone Ledge, and S. of the Breakwater to 35 fms. (R.A.T., S.P.): Millbay Ch.; Asia Sh.; abundant (A.J.S., S.P.): Eddystone Gds. (E.J.A.).

Alcyonidium hirsutum (Fleming): T. Hincks, Hist. Brit. Mar. Polyzoa, p. 493 , fig.

Cawsand B. (T.H.T. $\left.{ }^{1}\right)$.

Alcyonidium mytili, Dalyell: T. Hincks, Hist. Brit. Mar. Polyzoa, p. 498 , fig. Plymouth (s.F.H.).

Alcyonidium parasiticum (Fleming): T. Hincks, Hist. Brit. Mar. Polyzoa, p. 502, fig. Plymouth, on Sertularia cupressina (s.F.H.).

\section{Flustrellidæ.}

Flustrella hispida (Fabricius): T. Hincks, Hist. Brit. Mar. Polyzoa, p. 506 , fig.

Very abundant everywhere between tide-marks, on Fucus, etc. (R.M.P.).

Breeding: early Feb.-mid. July; spermatozoa more abundant Feb.-Mar.; ova, Mar.-May ; larvæ, Apr.-July (R.M.P.).

\section{Vesiculariidæ.}

Amathia lendigera (Linnæus): T. Hincks, Hist. Brit. Mar. Polyzoa, p. 516 , fig.

Penlee-Rame Gds., common on Halidrys (R.M.P.). 
[Bryozoa-contd.: Amphineura]

Bowerbankia caudata (Hincks): T. Hincks, Hist. Brit. Mar. Polyzoa, p. 521 , fig.

The Cattewater (т.н.т.).

Bowerbankia imbricata (Adams): T. Hincks, Hist. Brit. Mar. Polyzoa, p. 519 , fig.

Millbay Dock (т.н.т.).

Breeding: Aug. (A.J.S.).

Bowerbankia pustulosa (Ellis \& Solander): Tr. Hincks, Hist. Brit.

Mar. Polyzoa, p. 522, fig.

Plymouth, very common (s.F.H.): Duke Rk. (т.н.т.).

\section{Cylindrœciidæ.}

Cylindrecium dilatatum (Hincks): T. Hincks, Hist. Brit. Mar. Polyzoa, p. 536 , fig.

Eddystone Gds. (E.J.A.).

\section{Hypophorellidæ.}

Hypophorella expansa, Ehlers: J. Joyeux-Laffuie, Arch. Zool. expt., ser. 2, vol. vi. p. 152, fig. (as Delagia chatopteri).

Not uncommon on the tubes of Chatopterus variopedatus (s.F.H.).

Triticellidæ.

Triticella Boecki: G. O. Sars, Forhandl. vid.-Selsk. Christiania, vol. 1873 , p. 397, fig.

Rame-Eddystone Gds., on a spec. of Gonoplax rhomboides (R.A.T. ${ }^{1}$ ).

\section{Pedicillinidx.}

Pedicillina cernua (Pallas): T. Hincks, Hist. Brit. Mar. Polyzoa, p. 565 , fig.

Not uncommon, L.W.-35 fms.; Tinside; Asia Sh.; Drake's I.; Queen's Gd.; Duke Rk.; Wembury B.; Rame-Eddystone Gds., on Hydroids, Cellaria, Algæ, etc. (R.A.T.): Millbay Dock (т.H.T.): Penlee-Rame Gds., on Bowerbankia imbricata (R.M.P.): Eddystone Gds. (E.J.A.).

Pedicillina gracilis, Sars: T. Hincks, Hist. Brit. Mar. Polyzoa, p. 570 , fig.

From $5 \mathrm{~m}$. S. of the Mewstone, 27 fms. (т.н.т.).

\section{AMPHINEURA.}

Neomeniidæ.

Rhopalomenta Aglaophenia (Kovalevsky \& Marion): G. Pruvot, Arch. Zool. expt., ser. 2, vol. ix, p. 720, fig. (as Proneomenia).

Common on Aglaophenia myriophyllum, generally coiled around the base of the stem of the Hydroid (S.P., E.J.A.).

Mrzomenia banyulensis: G. Pruvot, Arch. Zool. expt., ser. 2, vol. ix,

p. 715, fig. (as Dondersia).

Occasionally on Lafoea dumosa (R.A.T., S.P., E.J.A.).

Gonads ripe: Aug. (R.A.T.).

NEW SERIES. - VOL. VII. No. 2. 
Ischnochitonidæ.

[Amphineura-contd.: Prosobranchiata]

Callochiton lavis (Montagu): J. G. Jeffieys, Brit. Conch., vol. iii, p. 226, fig. (as Chiton).

The Sound, under stones at low tide (s.P.).

Craspedochilus cinereus (Linnæus): E. Forbes \& Hanley, Hist. Brit.

Moll., vol. ii, p. 402, fig. (as Chiton).

The Sound, under stones at low tide; Yealm R., abundant on the sand-bank (s.P.).

Craspedochilus onyx (Spengler): E. Forbes \& Hanley, Hist. Brit. Moll., vol. ii, p. 407, fig. (as Chiton asellus).

Abundant, 15-30 fms., especially on muddy gravel (s.P.).

\section{Acanthochitidæ.}

Acanthochites fascicularis (Linnæus): E. Forbes \& Hanley; Hist. Brit. Moll., vol. ii, p. 393, fig. (as Chiton).

Rocks under the Hoe, moderately common among barnacles; Millbay Ch.; Mewstone Ledge ${ }^{1}$; Yealm R., occasionally in dredgings (R.A.T.): Blackstone Rks., Wembury B., abundant; Yealm Sand-bank (s.P.).

\section{PROSOBRANCHIATA.}

Patellidæ.

Patella vulgata, Linnæus: J. G. Jeffreys, Brit. Conch.; vol, iii, p. 236, fig.

More or less abundant everywhere on rocks between tidemarks (R.A.T.) : Mt. Edgcumbe, very large specs. (A.J.S.).

Helcion pellucida (Linnæus): J. G. Jeffieys, Brit. Conch., vol, iii, p. 242 , fig.

On stems of Laminaria, common everywhere, especially Reny Rks. and the Bridge (R.A.T.).

\section{Acmæidæ.}

Acmea virginea (Müller): E. Forbes \& Hanley, Hist. Brit. Moll., vol. ii, p. 437.

More or less common under stones on all rocky shores, L.W.5 fms., gregarious; Drake's I., large specs. (s.P.): moderately common in dredgings from the Yealm (R.A.T.).

\section{Fissurellidæ.}

Emarginula conica, Schumacher: E. Forbes \& Hanley, Hist. Brit. Moll., vol. ii, p. 479 (as $E$. rosea).

Not uncommon, $10-30$ fms. (s.P.).

Emarginula fissura (Linnæus): E. Forbes \& Hanley, Hist. Brit. Moll., vol. ii, p. 477 , fig. (as E. reticulata and E. Mülleri).

Millbay Ch.; Queen's Gd. (s.P.): the Breakwater, between tidemarks (R.A.T.): Mewstone Ledge; occasional specs. on the outside grounds in 15-30 fms. (R.A.T., S.P.): Eddystone Gds., constantly present, but seldom numerous, on all grounds where shells are plentiful (E.J.A.). 
[Prosobranchiata - contd.]

Fissurella GrecA (Linnæus): E. Forbes \& Hanley, Hist. Brit. Moll., vol. ii, p. 469, fig. (as $F$. reticulata).

Occasional specs. on all rocky stations, L.W.-10 fms. (s.P.): occasionally between tide-marks; the Breakwater; Reny Rks.; the Mewstone; Church Reef, Wembury B.; not uncommon in dredgings from the Yealm R. (R.A.T.).

\section{Trochidæ.}

Eumargarita gremilandica (Chemnitz): E. Forbes \& Hanley, Hist. Brit. Moll., vol. ii, p. 528, fig. (as Trochus undulatus).

A single specimen, possibly merely an empty shell, $3 \mathrm{~m}$. S.W. Penlee Pt. (R.A.T.).

Gibbula cineraria (Linnæus): E. Forbes \& Hanley, Hist. Brit. Moll., vol. ii, p. 516, fig. (as Trochus).

Abundant nearly everywhere, between tide-marks-10 fms., under stones, on Zostera, Fucus, etc. (s.P.).

Gibbula magus (Linnæus): J. G. Jeffreys, Brit. Conch., vol. iii, p. 305, fig. (as Trochus).

Uncommon at Plymouth, occurring only on coarse shell-sand or gravel; Queen's Gd.; between the Knap and Panther Buoys, four very large specs. (S.P.): Millbay 'shell-gravel' Gd.; Mewstone 'Amphioxus' Gd. (R.A.T.).

Gibbula tumida (Montagu): E. Forbes \& Hanley, Hist. Brit. Moll.; vol. ii, p. 513, fig. (as Trochus).

Occasional specs. from the Rame-Eddystone, Eddystone, Stoke Pt., and other outside grounds (s.P.).

Gibbula umbilicata (Montagu): E. Forbes \& Hanley, Hist. Brit. Moll., vol. ii, p. 519, fig. (as Trochus).

Common at most stations, but not so abundant as G. cineraria, and occurs at a rather higher level, H.W.-3 fms. (s.P.).

Monodonta crassa (Montfort): J. G. Jeffreys, Brit. Conch., vol. iii, p. 317, fig. (as Trochus lineatus).

The distribution of this species at Plymouth appears to be a very limited one, but it is met with in some numbers upon restricted areas of the rocks, at and above high-water mark, at those stations where it occurs; N.E. Drake's I.; Blackstone Rks., Wembury B.; Yealm Sand-bank (S.P.).

Calliostoma granulatum (Born): E. Forbes \& Hanley, Hist. Brit. Moll., vol. ii, p. 499, fig. (as Trochus).

Moderately common on sandy gravel, 20-30 fms. (s.P.): Mewstone 'Echinoderm' Gd., common (R.A.T.): Eddystone Gds., on gravel with sand or muddy sand (E.J.A.).

Calliostoma striatum (Linnæus): E. Forbes \& Hanley, Hist. Brit. Moll., vol ii, p. 508, fig. (as Trochus).

Occasionally ; Cawsand B. ; Jennycliff B.; Yealm Est., common on Zostera (R.A.T.).

Calliostoma zizyphinus (Linnæus): E. Forbes \& Hanley, Hist. Brit. Moll., vol. ii, p. 491, fig. (as Trochus).

Common under stones and in crevices on all rocky shores at low water; a dwarf var. is not uncommon in the deeper water of the outside grounds (s.P.): Yealm R., a characteristic var. is common on the sand-bank (R.A.T.). 


\section{Turbinidæ.}

[ Prosobranchiata-contd.]

Phasianella pullus (Linnæus): E. Forbes \& Hanley, Hist. Brit. Moll., vol. ii, p. 538, fig.

More or less common everywhere on Algæ and Zostera, L.W.$10 \mathrm{fms}$; ; common among weed growing on ships'-bottoms, buoys, etc. (s.P.): Queen's Gd.; Jennycliff B.; Cawsand B.; etc. (R.A.T., s.P.).

\section{Littorinidæ.}

Lacuna divaricata (Fabricius): E. Forbes \& Hanley, Hist. Brit. Moll., vol. iii, p. 62, fig. (as $L$. vincta).

Common on Fucus-covered rocks near low-water mark (s.P.): rocks below the Hoe, large specs. moderately common; smaller specs. common on the Zostera in Jennycliff B., Cawsand B., and Yealm Est.; $1 \mathrm{~m}$. S. of the Mewstone, very occasionally (R.A.T.).

Breeding: Feb.-Apr. (R.A.T.).

Lacuna pallidula (da Costa): E. Forbes \& Hanley, Hist. Brit. Moll., vol. iii, p. 56, fig.

Not uncommon on Fucus-covered rocks under the Hoe (R.A.T.).

Breeding: Feb. (R.A.T.).

Lacuna Parva (da Costa): E. Forbes \& Hanley, Hist. Brit. Moll., vol. iii, p. 58, fig. (as L. puteolus).

Cawsand B. (R.A.T., S.P.).

Littorina littorea (Linnæus): J. G. Jeffreys, Brit. Conch., vol. iii, p. 368, fig.

Not uncommon between tide-marks on most shores, but seldom in any quantity; this form occurs at a lower zone than $L$. rudis (s.P.): St. John's Lake, abundant; Yealm R., common (A.J.S.).

Breeding: Feb. (w.G.).

LitTorina neritoides (Linnæus): J. G. Jeffreys, Brit. Conch., vol. iii, p. 361, fig.

Abundant on rocks above high-water mark (s.P.).

Littorina obtusata (Linnæus): J. G. Jeffreys, Brit. Conch., vol. iii, p. 356 , fig.

Abundant everywhere on Fucus, Zostera, etc. (s.P.).

Breeding: Feb. (w.G.): Mar. (s.P.).

LitTorina RUdis (Maton): J. G. Jeffreys, Brit. Conch., vol. iii, p. 364, fig.

Very abundant on all rocky shores near high-water mark (s.P.).

\section{Rissoiidæ.}

Rissola parva (da Costa): E. Forbes \& Hanley, Hist. Brit. Moll., vol. iii, p. 98 , fig.

Moderately common in the Sound between tide-marks; occasionally in dredgings from Millbay Ch., Asia Sh., etc. (R.A.T.).

Alvania cancellata (da Costa): E. Forbes \& Hanley, Hist. Brit. Moll., vol. iii, p. 80, fig. (as Rissoa crenulata).

Dead shells only (R.A.T.).

Alvania reticulata (Montagu): E. Forbes \& Hanley, Hist. Brit. Moll., vol. iii, pp. 82 and 84, fig. (as Rissoa calathus and $R$. Beanii).

Dead shells only (R.A.T.) 
[Prosobranchiata-contd.]

Manzonia costata (J. Adams): E. Forbes \& Hanley, Hist. Brit. Moll., vol. iii, p. 92, fig. (as Rissoa).

Drake's I.; Millbay Pit; occasionally (R.A.T.).

Zippora membranacea (J. Adams): E. Forbes \& Hanley, Hist. Brit. Moll., vol. iii, p. 109, fig. (as Rissoa labiosa).

Common on Zostera (s.P.): Cawsand B., very common; Yealm Est., moderately common (R.A.T.).

Onoba striata (J. Adams): E. Forbes \& Hanley, Hist. Brit. Moll., vol. iii, p. 94, fig. (as Rissoa).

Common on all rocky shores, gregarious under stones, particularly where there is a certain amount of silt, L.W.-10 fms. (s.P.).

Ceratia proxima, Alder: E. Forbes \& Hanley, Hist. Brit. Moll., vol. iii, p. 127, fig. (as Rissoa).

Dead shells only (R.A.T.).

Hyala vitrea (Montagu): E. Forbes \& Hanley, Hist. Brit. Moll., vol. iii, p. 125 , fig. (as Rissoa).

Dead shells only (R.A.T.).

Cingula semistriata (Montagu): E. Forbes \& Hanley, Hist. Brit. Moll., vol. iii, p. 117, fig. (as Rissoa).

Millbay Pit (R.A.T.).

Cingula trifasciata, J. Adams: E. Forbes \& Hanley, Hist. Brit. Moll., vol. iii, p. 122, fig. (as Rissoa cingillus).

Common between tide-marks on all rocky shores, gregarious under stones and in crevices, especially where there is a certain amount of silt; Yealm Sand-bank (s.P.).

Galeodina carinata (da Costa): E. Forbes \& Hanley, Hist. Brit. Moll., vol. iii, p. 73, fig. (as Rissoa striatula).

Dead shells (R.A.T.). Assimineidæ.

Paludestrina stagnalis (Baster): J. G. Jeffreys, Brit. Conch., vol. iv, p. 52, fig. (as Hydrobia ulvae).

Common in brackish water; the Hamoaze; Hooe Lake; etc. (R.A.T.).

\section{Adeorbidæ.}

Adeorbis subcarinatus (Montagu): E. Forbes \& Hanley, Hist. Brit. Moll., vol. ii, p. 541, fig.

Ram's Cliff Pt., common on rocks at low tide, Aug. 1887 (w.H.).

\section{Skeneidæ.}

Skenea Planorbis (Fabricius): E. Forbes \& Hanley, Hist. Brit. Moll., vol. iii, p. 156, fig.

Common among the roots of seaweeds, and corallines, rocks under the Hoe; Drake's I. ; etc. (R.A.T.).

\section{Capulidæ.}

Capulus hungaricus (Linnæus): J. G. Jeffreys, Brit. Conch., vol. iii, p. 269 , fig.

Occasionally on gravel grounds, $15-35$ fms., generally attached to Pecten opercularis (s.P.): Mewstone Gds., occasionally on P. opercularis (R.A.T., S.P.): Eddystone Gds. (E.J.A., S.P.).

Breeding: Jan.-Mar. (w.G.). 
Calyptraa chinensis (Linnæus): J. G. Jeffreys, Brit. Conch., vol. iii, p. 273 , fig.

The Sound, common in dredgings on stony ground, attached to stones, shells, etc.; Asia Sh.; Millbay Ch.; the Bridge; etc. (S.P.):

Yealm R., always present in dredgings; Cawsand B.; etc. (R.A.T.):

Yealm Sand-bank, between tide-marks (s.P.).

Breeding: July (R.A.T.): Aug.-Sept. (S.P.).

\section{Cypræidæ.}

Trivia europala (Montagu): E. Forbes \& Hanley, Hist. Brit. Moll., vol. iii, p. 495, fig. (as Cyproea).

More or less abundant everywhere, particularly on rocky or stony ground, L.W. -30 fms.; the form occurring in deeper water is usually small and has a smooth, fawn-coloured mantle (S.P.).

Ovula (Simnia) patula (Pennant): J. G. Jeffreys, Brit. Conch., vol. iv, p. 407, fig.

Not uncommon on Alcyonium digitatum, 10-30 fms.; Mewstone Ledge; Stoke Pt. Gds. ; etc. (s.P.) : Mewstone Ledge, on Eunicella ${ }^{1}$ (W.I.B.).

Spawn probably belonging to this species has been found in Apr., June-July (R.A.T.).

Erato Levis (Donovan): J. G. Jeffreys, Brit. Conch., vol. iv, p. 400, fig. (as Marginella).

Not uncommon on gravel grounds, $15-30$ fms. (s.P.).

\section{Naticidæ.}

Natica (Lunatia) Alderi, Forbes: J. G. Jeffreys, Brit. Conch., vol. iv, p. 224, fig.

Not uncommon on clean sand and gravel, L.W.-35 fms. (s.P.): Drake's I., sand-patch at N. end, occasionally; Cawsand B., not uncommon; Mewstone 'Echinoderm' Gd.; Rame-Eddystone Gds. (R.A.T., S.P.): Stoke Pt. Gds. (S.P.): Eddystone Gds. (E.J.A., S.P.): Yealm R., on the sand-bank opposite the Coastguard Station (R.A.T.). Breeding: June (R.A.T.).

Natica (Lunatia) catena (da Costa): J. G. Jeffreys, Brit. Conch., vol. iv, p. 220 , fig.

On sandy bottoms (W.G.): rare (s.P.).

Breeding: Apr. (w.G.).

\section{Lamellariidæ.}

Lamellaria perspicua (Linnæus): E. Forbes \& Hanley, Hist. Brit. Moll., vol. iii, p. 355, fig. ; p. 338, fig. (as L. tentaculata).

Not uncommon between tide-marks and in dredgings from the Sound, etc. (S.P.): occasionally on the outside grounds, $15-30 \mathrm{fms}$. (R.A.T., W.I.B., S.P.) : Yealm R., not uncommon (R.A.T., S.P.).

Spawn : Jan.-May (w.G.).

Velutina Lavigata (Pennant): J. G. Jeffreys, Brit. Conch., vol. iv, p. 240 , fig.

Mewstone 'Echinoderm' Gd. (R.A.T.). 


\section{Cerithiidæ.}

Bittium reticulatum (da Costa): J. G. Jeffreys, Brit. Conch., vol. iv, p. 258, fig. (as Cerithium).

Not uncommon under stones on rocky shores, L.W.-10 fms. (s.P.).

Triforis perversa (Linnæus): E. Forbes \& Hanley, Hist. Brit. Moll., vol. iii, p. 195, fig. (as Cerithium adversum).

Occasional specimens not uncommon; Eddystone Gds.; RameEddystone Gds.; Mewstone Gds. ; Stoke Pt. Gds. (s.P.).

Cerithiopsis tubercularis (Montagu): E. Forbes \& Hanley, Hist. Brit. Moll., vol. iii, p. 365, fig.

Not uncommon in dredgings from the Sound, generally on sponges; occasionally on the outside grounds in 15-30 fms.; Yealm R., common on red sponge (R.A.T.).

\section{Scalidæ.}

Scala clathratula (Adams): E. Forbes \& Hanley, Hist. Brit. Moll., vol. iii, p. 209, fig. (as Scalaria).

Dead shells only (R.A.T.).

Scala clathrus (Linnæus): E. Forbes \& Hanley, Hist. Brit. Moll., vol. iii, p. 206, fig. (as Scalaria communis).

Rum B., ${ }^{1}$ between tide-marks; Asia Sh. ${ }^{1}$; Yealm R. ${ }^{1}$ (R.A.T.).

Scala Turtoni (Turton): E. Forbes \& Hanley, Hist. Brit. Moll., vol. iii, p. 204, fig. (as Scalaria).

Stoke Pt. Gds. ${ }^{1}$ (s.P.).

\section{Pyramidellidæ.}

Brachystomia ambigua (Maton \& Rackett): J. G. Jeffreys, Brit. Conch., vol. iv, p. 124, fig. (as Odostomia pallida).

Common on the ears of Pecten opercularis (R.A.T., S.P.): on P. maximus (R.A.T.).

Turbonilla lactea (Linnæus): J. G. Jeffreys, Brit. Conch., vol. iv, p. 167, fig. (as Odostomia).

Occasionally under stones, particularly where there is a certain amount of silt, L.W.-10 fms.; Drake's I.; Jennycliff B.; etc. (S.P.): Asia Sh.; Wembury B. (R.A.T., S.P.).

Pyrgostelis interrupta (Totten): E. Forbes \& Hanley, Hist. Brit. Moll., vol. iii, p. 245 , fig. (as Chemnitzia rufa).

Rame-Eddystone Gds., occasionally (S.P.).

\section{Eulimidæ.}

Eulima polita (Linnæus): J. G. Jeffreys, Brit. Conch., vol. iv, p. 201, fig.

Not uncommon on muddy gravel, 15-35 fms. (s.P.): Mewstone 'Amphioxus' Gd,, not uncommon; Mewstone Ledge, occasionally; Stoke Pt. Gds.; Rame-Eddystone Gds. (R.A.T., S.P.): Eddystone Gds. (E.J.A., S.P.).

Eulima incurva (Renier) $[=E$. distorta, auctt., nec Deshayes]: $E$. $R$. Sykes, Proc. Malac. Soc., vol. v, p. 350, fig.

Common among sponges, etc.; Millbay Pit; Asia Sh.; Mallard Sh.; Yealm R.; etc. (s.P.). 
[Prosobranchiata-contd.]

Eulima (Leiostraca) bilineata (Alder): E. Forbes \& Hanley, Hist. Brit. Moll., vol. iii, p. 237, fig.

Occasionally; Rame-Eddystone Gds.; Eddystone Gds.; etc. (s.P.).

Eulima (Leiostraca) glabra (da Costa): E. Forbes \& Hanley, Hist.

Brit. Moll., vol. iii, p. 235, fig. (as E. subulata).

Occasionally; Millbay Pit; Mewstone Gds.; etc. (s.P.).

\section{Cæcidæ.}

Cemcum glabrum (Montagu): E. Forbes \& Hanley, Hist. Brit. Moll., vol. iii, p. 181, fig.

Dead shells only (R.A.T.).

Cacum imperforatum (G. Adams): E. Forbes \& Hanley, Hist. Brit. Moll., vol. iii, p. 178, fig. (as C. trachea).

Dead shells only (R.A.T.).

\section{Turritellidæ.}

Turritella communis, Lamarck: J. G. Jeffreys, Brit. Conch., vol. iv, p. 80, fig. (as T. terebra).

Common in places, on muddy and sandy gravel, 15-30 fms. (s.P.): Mewstone Gds. (R.A.T., S.P.): Rame-Eddystone Gds.; Stoke Pt. Gds. (S.P.): Eddystone Gds. (E.J.A., S.P.).

\section{Aporrhaidæ.}

Aporrhais pes-Pelecani (Linnæus): J. G. Jeffreys, Brit. Conch., vol. iv, p. 250 , fig.

Not uncommon, particularly on muddy gravel, 5-35 fms.; Jennycliff B., rare; Cawsand B., occasionally; Rame-Eddystone Gds.; Stoke Pt. Gds.; etc. (S.P.): Eddystone Gds. (E.J.A., S.P.).

Young shells in Aug. (A.J.S.).

\section{Buccinidæ.}

Buccinum undatum (Linnæus): J. G. Jeffieys, Brit. Concb., vol. iv, p. 285 , fig.

Mewstone 'Echinoderm' Gd., moderately common; RameEddystone Gds.; Yealm R., moderately common in dredgings, and not uncommon on the sand-bank (R.A.T.): Eddystone Gds., numerous on the gravel and coarser sands near the Eddystone (E.J.A.): Stoke Pt. Gds. (s.P.).

Breeding: Jan.-Apr. (w.G.). Hatching: Feb.-Mar. (R.A.T.).

Donovania minima (Montagu): J. G. Jeffreys, Brit. Conch., vol. iv, p. 313 (as Lachesis).

Not uncommon in the Sound, under stones, L.W.-20 fms. (s.P.).

Tritonofusus gracilis (Costa): E. Forbes \& Hanley, Hist. Brit. Moll., vol. iii, p. 416, fig. (as Fusus islandicus).

Dead shells only (R.A.T.).

\section{Muricidæ.}

Ocinebra erinacea (Linnæus): J. G. Jeffreys, Brit. Conch., vol. iv, p. 306, fig. (as Murex).

Moderately common on rocks between tide-marks (R.A.T., S.P.): Asia Sh.; Millbay Ch.; Yealm R.; occasionally (R.A.T.).

Spawn: Apr. (R.A.T., S.P.): May (w.G.). 
[Prosobranchiata-contd.]

Trophon muricatus (Montagu): E. Forbes \& Hanley, Hist. Brit. Moll., vol. iii, p. 439.

Occasionally taken in 15-30 fms.; not uncommon on the Mewstone 'Echinoderm' Gd. (R.A.T.).

Purpura lapillus (Linnæus): J. G. Jeffreys, Brit. Conch., vol. iv, p. 276 , fig.

Abundant on rocks between tide-marks everywhere (s.P.).

Spawn: Jan.-Apr. (w.G., R.A.T.): May (w.G.): July (s.P.): Nov. (w.G.).

\section{Nassidæ.}

Nassa reticulata (Linnæus): E. Forbes \& Hanley, Hist. Brit. Moll., vol. iii, p. 388, fig.

More or less common everywhere, L.W.-5 fms., especially on muddy sand; Cawsand B.; Jennycliiff B.; Drake's I.; Yealm R. ; etc. (S.P.).

Breeding: Feb. (w.G.): Mar. (w.G., R.A.T., S.P.): Apr. (w.G., R.A.T.): May (s.P.): June (R.A.T., S.P.): July (w.G.): Aug. (s.P.): Sept. (w.G.).

Nassa incrassata (Ström): E. Forbes \& Hanley, Hist. Brit. Moll., vol. iii, p. 391, fig.

More or less common on all rocky shores, gregarious under stones and in crevices, particularly where there is a certain amount of silt, L.W.-10 fms. (S.P.).

Breeding: Feb.-Mar. (s.P.) : Apr. (w.G., R.A.T., s.P.) : June (w.g.): Aug. (w.G., S.P.): Sept. (W.G.).

\section{Pleurotomidæ.}

Mangilia attendata (Montagu): J. G. Jeffreys, Brit. Conch., vol. iv, p. 377, fig. (as Pleurotoma).

Occasionally in muddy gravel, $20-30$ fms. (s.P.).

Mangilia nebula (Montagu): E. Forbes \& Hanley, Hist. Brit. Moll., vol. iii, p. 476, fig.

Queen's Gd. (R.A.T. $\left.{ }^{1}\right)$.

Mangilia (Bellardiella) gracilis, P. Fischer: J. G. Jeffreys, Brit. Conch., vol. iv, p. 363, fig. (as Defrancia).

Not uncommon, 20-35 fms., particularly on muddy gravel (s.P.).

Clathurella linearis (Montagu): $J$. G. Jeffireys, Brit. Conch., vol. iv, p. 368, fig. (as Defrancia).

Queen's Gd., not uncommon; Asia Sh.; occasionally on the outside grounds, $15-30$ fms. (R.A.T., S.P.): the Breakwater, occasionally (R.A.T.).

Clathurella purpurea (Montagu): J. G. Jeffreyz, Brit. Conch., vol. iv, p. 373, fig. (as Defrancia).

Occasionally under stones and in rock crevices, L.W.-5 fms.; Drake's I.; Asia Sh.; Hollow Rock B., Jennycliff B.; Blackstone Rks., Wembury B.; etc. (s.P.).

Breeding: Nov. (S.P.).

Clathurella reticulata (Renier): J. G. Jeffreys, Brit. Conch., vol. iv, p. 370, fig. (as Defrancia).

Occasionally, $15-30$ fms. (s.P.). 
[Tectibranchiata ]

TECTIBRANCHIATA.

\section{Actæonidæ.}

ACtaon tornatilis (Linnæus): J. G. Jeffreys, Brit. Conch., vol. iv, p. 433 , fig.

Dead shells occasionally taken (R.A.T.).

Tornatinidæ.

Tonnatina mamillata (Philippi): J. G. Jeffieys, Brit. Conch., vol. iv, p. 420, fig. (as Utriculus).

Dead shells occasionally taken (R.A.T.).

Tornatina truncatula (Bruguière): E. Forbes \& Hanley, Hist. Brit.

Moll., vol. iii, p. 510 (as Cylichna truncata).

Only dead shells (R.A.T.).

\section{Scaphandridæ.}

Scaphander lignarius (Linnæus): J. G. Jeffreys, Brit. Conch., vol. iv, p. 443 , fig.

Common on the trawling grounds outside Sound (W.I.B., S.P.).

Volvulella acuminata (Bruguière): J. G. Jeffreys, Brit. Conch., vol. iv, p. 411, fig. (as Cylichna).

Dead shell only (R.A.T.).

Bullinella cylindracea (Pennant): J. G. Jeffreys, Brit. Conch., vol. iv, p. 415, fig. (as Cylichna).

Dead shells only taken (R.A.T.).

\section{Bullidæ.}

Haminea hydatis (Linnæus): J. G. Jeffreys, Brit. Conch., vol. iv, p. 437, fig. (as Bulla).

Yealm Est. (w.G.): Jennycliff B. (R.A.T. $\left.{ }^{1}\right)$.

Roxania utriculus, Brocchi: E. Forbes \& Hanley, Hist. Brit. Moll., vol. iii, p. 533, fig. (as Bulla Cranchi).

A single specimen, $4 \frac{1}{2} \mathrm{~m}$. S.W. by W. Penlee Pt. (R.A.T.).

\section{Philinidæ.}

Philine aperta (Linnæus): J. G. Jeffreys, Brit. Conch., vol. iv, p. 457, fig.

Common on muddy sand bottom, at times very abundant; Cawsand B.; Jennycliff B.; Cattewater, etc. (W.I.B., S.P.) : sandy bottom Whitsand B. (w.G.).

Breeding: Apr. (w.G.): May-July (w.G., R.A.T.).

Philine Catena (Montagu): J. G. Jeffreys, Brit. Conch., vol. iv, p. 449, fig.

Mewstone Ledge (w.I.B. $\left.{ }^{1}\right)$ : Millbay Ch. (R.A.T.).

Philine punctata (J. Adams): J. G. Jeffreys, Brit. Conch., vol. iv, p. 453 , fig.

The Sound, single spec. among Bowerbankia; plentiful 20 fms., among shells covered with Bugula, 1891 (w.G.): Drake's I. (E.J.A. ${ }^{1}$ ): Asia Sh. ${ }^{1}$; Millbay Docks, occasionally on the piles (R.A.T.).

Philine scabra (Müller): J. G. Jeffreys, Brit. Conch., vol. iv, p. 447, fig. Whitsand B., 4-12 fms. (w.H. ${ }^{1}$ ). 
[Tectibranchiata-contd: Nudibranchiata]

Colpodaspis pusilla, M. Sars: W. Garstang, Proc. Zool. Soc., vol. 1894, p. 664, fig.

On rough ground, about $2 \mathrm{~m}$. S. of the Mewstone, Feb. 1893 (w.G.).

\section{Aplysiidæ.}

Aplysia punctata, Cuvier: W. Garstang, Journ. Mar. Biol. Assoc., ser. 2, vol, i, p. 401.

Queen's Gd., rare; Cattewater; Cawsand B.; Reny Rks.; Yealm R., moderately common in dredgings, very common at times on the shore, especially in May and June; Yealm Est., small specs. not uncommon on the Zostera (R.A.T.): E. end of Whitsand B., in quantity beneath seaweed on rocks (w.H.).

Breeding: Apr.-July (R.A.T.): June-Oct. (w.G.): maximum, May-June (A.J.S.).

\section{Pleurobranchidæ.}

Pleurobranchus plumula (Montagu): J. G. Jeffreys, Brit. Conch., vol. v, p. 11, fig.

Jennycliff B. (A.J.S.): Wembury B., on the shore; S. of the Mewstone (w.G.).

Oscanius membranaceus (Montagu): J. G. Jeffreys, Brit. Conch., vol. v, p. 10, fig. (as Pleurobranchus).

Net uncommon on the trawling grounds (W.I.B., s.P.): the Sound, unusually plentiful, especially in Millbay Ch. and the Hamoaze, 1893; young specs. common swimming at the surface in the Sound, Sept. 1892 (w.G.): Cattewater and Yealm R., very occasional (R.A.T.).

\section{Runcinidæ.}

Runcina coronata (Quatrefages): J.G. Jeffreys, Brit. Conch., vol. v, p. 15, fig. (as $R$. Hancocki).

Tide-pools below Hoe, very abundant Apr. 1889, small specs. in Sept. (W.G.): Rum B. (R.A.T. ${ }^{1}$ ): among corallines from rock-pools in the Sound; Yealm R. (w.I.B.).

\section{NUDIBRANCHIATA.}

Hermæidæ.

Herma BIfIDA (Montagu): J. Alder \& Hancock, Monogr. Brit. Nudibr. Moll., p. 53, fig.

St. Peter's Pt., Hamoaze, single spec. on Delesseria (w.G.): West Entrance of Sound, in townet, single spec.; Cawsand B., single spec. (w.I.B.) : off Penlee Pt., small spec. in townet (S.P.).

Hermat dendritica (Alder \& Hancock): J. Alder \& Hancock, Monogr. Brit. Nudibr. Moll., p. 53, fig.

Drake's I., two on Bryopsis (w.G.): inside Bovisand Pier, single spec. (R.A.T.): in Lab. tank on a stone from Bovisand B. (W.I.B.).

Breeding: June (w.G.).

StIliger Bellulus, d'Orbigny.

Cawsand B. (w.G., ${ }^{1}$. 


\section{Elysiidæ.}

[Nudibranchiata—contd.]

Elysia viridis (Montagu): E. Forbes \& Hanley, Hist. Brit. Moll., vol. iii, p. 614 , fig.

Yealm Est., common (G.C.B., w.G.): Duke Rk. ${ }^{1}$; middle of the Sound ${ }^{1}$; tide-pools below the Hoe ${ }^{1}$ (w.G.): Queen's Gd.; Yealm R.; uncommon (w.I.B.).

Breeding: Oct., and probably earlier (w.G.).

\section{Limapontiidæ.}

Limapontia capitata (Müller): J. Alder \& Hancock, Ann. Mag. Nat. Hist., ser. 2, vol. i, p. 402, fig. (as L. nigra).

Reny Rks. (w.H., w.G.): Cawsand B., abundant in tide-pools (w.G.): tide-pools below the Hoe, abundant Apr. 1890, disappeared Aug. and Sept. (w.G.): Drake's I., common on corallines (R.A.T., w.G.): Rum B., occasionally (R.A.T., S.P.).

Acteonia corrugata: J. Alder \& Hancock, Ann. Mag. Nat. Hist., ser. 2, vol. i, p. 403, fig.

Tide-pools below the Hoe (w.G. ${ }^{1}$ ): Jennycliff B. (s.P.): Bovisand B., single spec. among corallines (w.I.B.).

\section{Æolidiidæ.}

Folidia papillosa (Linnæus): J. Alder \& Hancock, Monogr. Brit. Nudibr. Moll., p. 48, fig. (as Eolis).

Fairly common on all stony shores (w.I.B., S.P.): Yealm R., specs. common and particularly fine, among heaps of drift Fucus, etc. (A.J.S.).

Breeding: Feb. (R.A.T.): Mar.-May (w.G., R.A.T.): June (w.G.): July-Aug. (R.A.T.).

Aolidella Alderi (Cocks): J. Alder \& Hancock, Monogr. Brit. Nudibr. Moll., p. 48, fig. (as Eolis).

Drake's I.; Rocks below the Hoe ${ }^{1}$ (E.J.A.): Yealm R. (w.I.B., w.G., R.A.T.): Reny Rks. (R.A.T.1): Wembury B., among corallines (W.I.B.).

Breeding: Aug. (w.G.).

Folidella glauca (Alder \& Hancock): J. Alder \& Hancock, Monogr. Brit. Nudibr. Moll., p. 48, fig. (as Eolis).

Millbay Ch., occasionally (R.A.T., W.I.B., A.J.S., S.P.): Drake's I., rarely (R.A.T., S.P.): Mallard Sh. (J.c.s. $\left.{ }^{1}\right)$ : Mt. Edgeumbe (w.G. ${ }^{1}$ ): Queen's Gd. (w.I.B., R.A.T.): Asia Sh. (S.P.): Cattewater, large spec. probably from trawl refuse; 5-6 m. S.W. Breakwater Lt.; Mewstone Ledge (W.I.B.) : several specs. under Laira Bridge (A.J.S.).

Berghia Carulescens (Guérin Méneville): R. Bergh, Verhandl. Zool.bot. Ges. Wien, vol. xxxii, p. 20.

Outside the Breakwater (w.G.).

Cuthona Peachi (Alder \& Hancock): J. Alder \& Hancock, Monogr. Brit. Nudibr. Moll., p. 50, fig. (as Eolis).

Two specs. with spawn on Hydractinia, 3-4 m. S. of the Mewstone (W.I.B.).

Cavolina amena (Alder \& Hancock): J. Alder \& Hancock, Monogr. Brit. Nudibr. Moll., p. 51.

Occasionally on stony ground; Millbay Ch.; Asia Sh.; New Gds. (w.I.B.). 
[ Nudibranchiata - contd.]

Cavolina aurantia (Alder \& Hancock): J. Alder \& Hancock, Monogr.

Brit. Nudibr. Moll., p. 51, fig. (as Eolis).

On piles, Millbay Dock ${ }^{1}$; buoy near the Breakwater ${ }^{1}$ (W.I.B. \&

R.A.T.): Millbay Ch., very rare, one on Garveia nutans (R.A.T., W.I.B.):

Duke Rk. (w.I.B.).

Breeding: May (w.I.B., R.A.T.).

Cavolina olivacea (Alder \& Hancock): J. Alder \& Hancock, Monogr.

Brit. Nudibr. Moll., p. 51, fig.

Occasionally on stony and rocky ground (w.I.B.): Queen's Gd., occasionally (w.I.B., R.A.T., S.P.): Asia Sh.; Drake's I.; Millbay Ch. and Pit (R.A.T.): Mewstone Gds. (w.G. ${ }^{1}$ ): Yealm R. (w.I.B.): Mewstone Ledge (W.I.B., S.P.).

Cratena viridis (Forbes): J. Alder \& Hancock, Monogr. Brit. Nudibr. Moll., p. 51, fig. (as Eolis).

Millbay Ch., single spec. (A.J.S. ${ }^{1}$ ): Duke Rk. (w.G. ${ }^{1}$ ): Mewstone Gds. (w.G.): Rame-Eddystone 'Cellaria' Gds. (w.I.B.): Eddystone Gds. (E.J.A.).

Tergipes Despectus (Johnston): J. Alder \& Hancock, Monogr. Brit. Nudibr. Moll., p. 52, fig.

Millbay Dock, on piles, common, Mar. 1901 (R.A.T.): Barn Pool (w.I.B.): buoy near Breakwater; Duke Rk. (R.A.T.): Mewstone Gds. (W.G.).

Breeding: Mar. (w.G., R.A.T.): Apr.-May (R.A.T.).

Embletonia pulchra (Alder \& Hancock) : J. Alder \& Hancock, Monogr.

Brit. Nudibr. Moll., p. 52, fig.

Asia Sh., single spec., apparently on Antennularia (w.I.B.).

Amphorina c.ærulea (Montagu): J. G. Jeffreys, Brit. Conch., vol. v, p. 51 (as Eolis).

Asia Sh.; Queen's Gd., not uncommon (s.P.): Rame-Eddystone Gds.; Mewstone-Eddystone Gds., on 'Cellaria' grounds (w.I.B.); Mewstone Ledge; off Tregantle (w.I.B. \& R.A.T.).

Galvina cingulata, Alder \& Hancock: J. Alder \& Hancock, Monogr. Brit. Nudibr. Moll., p. 51, fig. (as Eolis).

From among weed, etc., below the Laboratory (w.I.B.): Asia Sh.'

(w.I.B. \& R.A.T. ${ }^{1}$ ): Millbay Dock, on Plumularia from the piles;

Yealm Est., on Zostera; Yealm R., on Antennularia (w.I.B.):

Mewstone Gds. (w.G. ${ }^{1}$ ).

Breeding: July (w.G.).

Galvina exigua (Alder \& Hancock): J. Alder \& Hancock, Monogr.

Brit. Nudibr. Moll., p. 52, fig. (as Eolis).

Duke Rk. (w.G.): Barn Pool, several spees, on Obelia (w.I.B.).

Breeding: Mar. (w.G.).

Galvina PICTA, Alder \& Hancock: J. Alder \& Hancock, Monogr. Brit.

Nudibr. Moll., pp. 51, 52, fig. (as Eolis picta and E. Farrani).

Cawsand B., common on Zostera; Asia Sh.; Millbay Ch.; Queen's

Gd.; Yealm Est., on Zostera (w.I.B.): buoy near West Hoe, among Tubularia (s.P.). 
[Nudibranchiata-contd.]

Galvina tricolor (Forbes): J. Alder \& Hancock, Monogr. Brit. Nudibr. Moll., p. 51, fig. (as Eolis).

Fairly abundant on the trawling grounds, $20-35$ fms. (W.I.B., S.P.). Breeding: May; Nov. (w.G.).

Coryphella Gracilis (Alder \& Hancock) : J. Alder \& Hancock, Monogr. Brit. Nudibr. Moll., p. 49, fig.

Single spec., between Duke Rk. and Jennycliff; Cawsand B., single spec. (W.G.).

Coryphella Landsburgi (Alder \& Hancock): J. Alder \& Hancock, Monogr. Brit. Nudibr. Moll., p. 49, fig. (as Eolis).

Occasionally at most stations in the Sound and outside (W.I.B., S.P.) : twice amongst Hydroids, Duke Rk. (w.G.).

Coryphella lineata (Lovén): J. Alder \& Hancock, Monogr. Brit. Nudibr. Moll., p. 49, fig. (as Eolis).

Mewstone Ledge, rare (R.A.T.): on stone dredged in Firestone B.; Rame-Eddystone Gds. (W.I.B.).

Coryphella rufibranchialis (Johnston): J. Alder \& Hancock, Monogr.

Brit. Nudibr. Moll., p. 49, fig.

Fairly common on the 'Inner' trawling grounds (w.I.B., s.P.): Whitsand B. (W.G.).

Coryphella smaragdina $[?=C$. gracilis, var.]: J. Alder \& Hancock,

Monogr. Brit. Nudibr. Moll., p. 49, fig.

Millbay Ch.; Asia Sh.; May 1898 (w.I.B.).

Favorinus albus (Alder \& Hancock): J. Alder \& Hancock, Monogr.

Brit. Nudibr. Moll., p. 49, fig.

Drake's I. (w.G., w.I.B.) : Asia Sh., on Antennularia; Barn Pool, on Fucus; Batten B.; Duke Rk. (w.I.B.): Cawsand B. (w.G.): Yealm Est., on Zostera, fairly common in 1897 (w.I.B.).

Breeding: June (w.I.B.) : Nov. (w.G.).

Facelina coronata, Forbes \& Goodsir: J. Alder \& Hancock, Monogr.

Brit. Nudibr. Moll., p. 49, fig. (as Eolis).

Millbay Dock, on piles; Millbay Ch.; Cawsand B. (w.I.B.); the

Cattewater; Cobbler Buoy; Duke Rk.; off the Mewstone (w.G.):

reef between Wembury Pt. and the Mewstone (E.J.A.).

Breeding: Apr.-May (w.G.).

Facelina Drummondi (Thompson): J. Alder \& Hancock, Monogr. Brit.

Nudibr. Moll., p. 49, fig. (as Eolis).

Queen's Gd. ; Cawsand B.; Yealm Est. (w.I.B.).

Facelina punctata (Alder \& Hancock): J. Alder \& Hancock, Monogr.

Brit. Nubibr. Moll., p. 49, fig. (as Eolis).

Millbay Ch.; Cawsand B.; rocky ground off Penlee (w.I.B.): Mewstone Gds. (w.G., w.I.B. \& R.A.T.).

Calma glaucoides (Alder \& Hancock): J. Alder \& Hancock, Monogr. Brit. Nudibr. Moll., p. 50, fig. (as Eolis).

Queen's Gd. (W.I.B. \& R.A.T. ${ }^{1}$ ): Blackstone Rks., Wembury B. (s.P.): West Entrance of Sound, amongst goby eggs, which is apparently its natural habitat (w.I.B.): Mewstone Gds., generally in Buccinum shells occupied by Blennius ocellaris, sometimes as many as 50 in 
[ Nudibranchiata_contd.]

one shell; resembles the eggs with which it is associated, the colour varying according to whether the eggs are those of the Goby or Butterfly Blenny (A.J.S.).

Antiopella cristata (delle Chiaje): J. Alder \& Hancock, Monogr. Brit. Nudibr. Moll., p. 54, fig. (as Antiopa).

Rock-pool under the Hoe; Rum B. (W.G.): Queen's Gd. (s.P., w.I.B.): Millbay Docks, very large spec.; Eddystone Gds. (w.I.B.) : Mewstone Gds. (w.G.): Yealm R. (R.A.T.).

Breeding: Aug. (R.A.T.).

Antiopella hyalina, Alder \& Hancock: $J$. Alder \& Hancock, Monogr.

Brit. Nudibr. Moll., p. 54, fig. (as Antiopa).

Queen's Gd., several small specs. (W.I.B.): Duke Rk. (т.v.H. ${ }^{1}$ ):

Mewstone Ledge (w.I.B. \& R.A.T. ${ }^{1}$ ): Mewstone 'Echinoderm' Gd.;

2-4 m. N.E. of Eddystone (w.I.B.).

Hero formosa (Lovén): J. G. Jeffreys, Brit. Conch., vol. v, p. 63.

Not uncommon outside the Breakwater (w.G.): Eddystone Gds.;

Mewstone Gds.; Stoke Pt. Gds.; 6 m. S. of the Mewstone, in considerable numbers, Jan.-June 1895; has not been seen since 1898 (E.J.A.).

Breeding : Apr. (w.G.).

\section{Lomanotidæ.}

Lomanotus marmoratus (Alder \& Hancock): W. I. Beaumont, Proc. Irish Acad., ser. 3, vol. v, p. 846.

Fairly common on Antennularia in shallow water; Asia Sh.;

New Gds.; Millbay; etc. (w.I.B., s.P.): about $1 \mathrm{~m}$. S. of Mewstone, large spec. (w.I.B.) : $3 \mathrm{~m}$. S. of Mewstone* (w.G.).

Lomanotus portlandicus, Thompson: $W$. I. Beaumont, Proc. Irish Acad., ser. 3, vol. v, p. 842.

Very scarce; two specs., New Gds.; 4 m. S. of the Mewstone, large spec.; 3 m. S.S.W. of Rame Hd. (w.I.в.): Queen's Gd., $†$ young specimens on Antennularia (w.G.).

Spawned: Sept. (w.I.B.).

\section{Dotonidæ.}

Doto coronata (Gmelin) : J. Alder \& Hancock, Monogr. Brit. Nudibr. Moll., p. 48, fig.

Fairly plentiful on Antennularia at most stations (w.I.B., s.P.): always on calyptoblastic Hydroids (w.G.): Millbay Docks, on Plumularia; buoy near Breakwater; Drake's I., on the shore (w.I.B.) : Yealm R. (W.I.B., R.A.T.).

Breeding: most of the year (w.I.B., s.P.).

Doto fragilis (Forbes): J. Alder \& Hancock, Monogr. Brit. Nudibr. Moll., p. 48, fig.

Common on Antennularia, etc., from the outside grounds, less frequent in the Sound (W.G., W.I.B., S.P.).

Breeding during the greater part of the year (W.I.B., S.P.).

$$
\text { * As L. varians. } \quad+\text { As L. Genei. }
$$


[Nudibranchiata_contd.]

Doto pinnatifida (Montagu): J. Alder \& Hancock, Monogr. Brit. Nudibr. Moll., p. 48, fig.

Generally distributed and common on Antennularia in the Sound and outside (W.I.B., S.P.).

Breeding most of the year (W.I.B., S.P.).

Hancockia eudactrlota, Gosse: $F$. W. Gamble, Journ. Mar. Biol. Assoc., ser. 2, vol. ii, p. 193 .

Between Drake's I. and Breakwater Lt. (F.w.G. ${ }^{1}$ ); Cawsand B.; Yealm Est.; on Zostera and on Laminaria, rare (w.I.B.).

\section{Dendronotidæ.}

Dendronotus frondosus (Ascanius): J. Alder \& Hancock, Monogr. Brit. Nudibr. Moll., p. 47, fig. (as D. arborescens).

Local specimens are usually small (s.P.): occasionally in the Sound (G.c.B.) : Queen's Gd. (s.P., w.I.B.): R. Tamar, near Saltash, a large spec. (w.I.B.): Mewstone Ledge occasionally (R.A.T.): MewstoneEddystone Gds. (W.G.): Eddystone Gds. (E.J.A.): Rame-Eddystone Gds. (w.G., S.P.).

\section{Pleurophyllidiidæ.}

Pleurophyllidia Loveni, Bergh : E. Forbes \& Hantey, Hist. Brit. Moll., vol. iv, p. 290, fig. (as Diphyllidia lineata).

Single spec., $2 \mathrm{~m}$. N. of Eddystone (J.T.c.).

\section{Tritoniidæ.}

Tritonia Hombergi, Cuvier : J. Alder \& Hancock, Monogr. Brit. Nudibr. Moll., p. 46, fig.

The habitat of this form appears to be below the $30 \mathrm{fm}$. line (w.I.B., s.P.): Mewstone Ledge (т.V.H.): common on the 'outer' trawling grounds (w.G.): Eddystone Gds. (E.J.A., W.G., S.P.): Mewstone Gds. (J.T.C.).

Tritonia (Candiella) lineata, Alder \& Hancock: J. Alder \& Hancock, Monogr. Brit. Nudibr. Moll., p. 47, fig.

Stoke Pt. Gds., single spec. (s.P.).

Tritonia (Candiella) Plebeia, Johnston : J. Alder \& Hancock, Monogr. Brit. Nudibr. Moll., p. 47, fig.

Millbay Pit (A.J.S.): Mewstone Gds. (w.G.): Rame-Eddystone Gds. (w.I.B.): Eddystone Gds. (E.J.A., W.I.B.).

A pink variety is fairly common on Eunicella, especially from the Mewstone Ledge (w.I.B.).

Breeding: Aug.; Oct. (R.A.t.) : Nov. (w.G.).

\section{Dorididæ.}

Archidoris flammea (Alder \& Hancock): J. Alder \& Hancock, Monogr. Brit. Nudibr. Moll., p. 41, fig. (as Doris).

Duke Rk., single spec. on red sponge (w.G.): Queen's Gd. or Duke Rk., single spec. (w.I.B.).

ARChidoris (gen.?) Maculata, Garstang: W. Garstang, Journ. Mar. Biol. Assoc., ser. 2, vol. iv, p. 167 (as Doris).

Queen's Gd. (w.G., S.P.): Mewstone Ledge (т.V.H.): Eddystone Gds. (E.J.A., W.I.B.). 
[ Nudibranchiata-contd.]

Archidoris tuberculata (Cuvier): J. Alder \& Hancock, Monogr. Brit. Nudibr. Moll., p. 41, fig. (as Doris).

Not uncommon on most rocky shores and in dredgings from the Sound (W.G., w.I.B., s.P.): Yealnı R.; Rame-Eddystone Gds.; Eddystone Gds. (W.I.B.).

Breeding: Jan.-June (w.G.).

Rostanga coccinea (Forbes): J. Alder \& Hancock, Monogr. Brit. Nudibr. Moll., p. 42, fig. (as Doris).

Occasionally on the shore and in dredgings from the Sound on stony ground (w.I.B., s P.): Drake's I. (w.G., G.c B.): Yealm R.; Downderry, on the shore (w.I.B.)

Jorunna Johnstoni (Alder \& Hancock): J. Alder \& Hancock, Monogr. Brit. Nudibr. Moll., p. 42, fig. (as Doris).

Occasionally on rocky and stony shores, and in dredgings from the Sound (w.G., w.I.B., S.P.): Reny Rks.; Yealm Est., south shore (W.I.B.).

Platydoris (gen.?) testudinaria (Risso): J. G. Jeffreys, Brit. Conch., vol. v, p. 85 (as Doris).

Drake's I. (w.G., T.v.H.): Barn Pool (w.H., w.G.) : Garden Battery (E.J.A.): Mt. Edgcumbe; Millbay Ch.; Queen's Gd., very occasionally (R.A.T.): Duke Rk. (W.G.): Yealm R. (R.A.T.): Rame-Eddystone Gds. (W.I.B.).

\section{Polyceridæ.}

Ægires punctilucens (d'Orbigny): J. Alder \& Hancock, Monogr. Brit. Nudibr. Moll., p. 44, fig. (as AEgirus).

Millbay Pit and Ch., very occasionally; Drake's I., single spec. among coralline (R.A.T.): Queen's Gd. (T.V.H.): Duke Rk. (W.G., T.V.H.): Yealm R. (R.A.T.): Wembury B., few small specs. among corallines (T.V.H.): Eddystone Gds. (E.J.A.).

Triopa cla vigera (Müller): J. Alder \& Hancock, Monogr. Brit. Nudibr. Moll., p. 44, fig.

Millbay Pit, single spec. (w.I.B., R.A.T.): Mewstone Ledge, occasionally (R.A.T.) : Mewstone Gds. (w.G., E.J.A.): Stoke Pt. Gds.; Eddystone Gds. (E.J.A.).

Thecacera pennigera (Montagu) : J. Alder \& Hancock, Monogr. Brit. Nudibr. Moll., p. 44, fig.

Off Rame Hd., 20 fms., single spec. (w.G.): Queen's Gd. (s.P.).

Palio Lessoni (d'Orbigny): J. Alder \& Hancock, Monogr. Brit. Nudibr. Moll., p. 45, fig. (as Polycera ocellata and P. Lessonii).

Rum B. (W.I.B., R.A.T., S.P.): Duke Rk.; Mt. Edgcumbe; Drake's I. (w.G.): Queen's Gd. (T.V.H.): Asia Sh. (R.A.T.).

Polycera quadrilineata (Müller): J. Alder \& Hancock, Monogr. Brit. Nudibr. Moll., p. 45, fig.

In most years abundant on Zostera beds; Cawsand B.; Yealm Est.; etc. (W.I.B., S.P.): Drake's I., under stones (W.I.B.): Yealm R., occasionally (R.A.T., W.I.B.): Queen's Gd.(W.I.B.): Wembury B. (W.G.): Rame-Eddystone Gds.; Eddystone Gds. (w.I.B., s.P.): Bigbury B., large spec. close in, off Thurlestone (W.I.B.).

Breeding: July-Aug. (w.G.).

NEW SERIES, - VOL, VII. NO, 2 , 
[Nudibranchiata-contd.]

Acanthodoris pilosa (Müller): J. Alder \& Hancock, Monogr. Brit, Nudibr. Moll., p. 43, fig. (as Doris).

Rocks under the Lab. (E.J.A.): Drake's I., occasionally (E.J.A., R.A.T.): Millbay Ch., occasionally; Asia Sh., occasionally; Queen's Gd., not uncommon; Rum B.; the Breakwater (R.A.T.): Duke Rk. (т.v.H.,R.A.T.): Bovisand B. (T.v.H.): Wembury B. (W.G.): Yealm R., not uncommon (R.A.T.): $2 \mathrm{~m}$. N. of the Eddystone (J.T.C.).

Breeding: May (A.J.S.).

LAMELlidoris aspera, Alder \& Hancock: J. Alder \& Hancock, Monogr. Brit. Nudibr. Moll., p. 42, fig. (as Doris).

Between the Mallard and Cobbler Buoys, single spec. (w.G.):

Yealm Est., very rare (R.A.T.).

Lamellidoris bilamellata (Linnæus): $J$. Alder \& Hancock, Monogr. Brit. Nudibr. Moll., p. 43, fig. (as Doris).

Common; Batten B.; Jeunycliff B.; Duke Rk. (w.G.): Millbay

Ch.; the Breakwater; Yealm R. (W.I.B., R.A.T.): $4 \mathrm{~m}$. N.E. of the Eddystone (T.V.H. $\left.{ }^{1}\right)$.

Breeding: Mar.-Apr. (R.A.T.).

LAMELlidoris DePressa (Alder \& Hancock): J. Alder \& Hancock, Monogr.

Brit. Nudibr. Moll., p. 43, fig.

Inner side of the Breakwater ${ }^{1}$; Mewstone Ledge ${ }^{1}$ (w.I.B.).

Lamellidoris oblonga, Alder \& Hancock: J. Alder \& Hancock, Monogr.

Brit. Nudibr. Moll., p. 43, fig. (as Doris).

Extremely abundant at times on Cellaria; Mewstone Ledge;

Rame-Eddystone Gds.; Eddystone Gds.; etc. (w.I.B.).

Breeding: Apr. (w.G.).

Lamellidoris pusilla, Alder \& Hancock: J. Alder \& Hancock, Monogr.

Brit. Nudibr. Moll., p. 43, fig.

Plymouth (w.G.).

Breeding: Feb. (w.G.).

Lamellidoris sparsa, Alder \& Hancock: J. Alder \& Hancock, Monogr.

Brit. Nudibr. Moll., p. 43, fig. (as Doris). Off Stoke Pt. (w.G. ${ }^{1}$ ).

Goniodoris castanea, Alder \& Hancock: J. Alder \& Hancock, Monogr. Brit. Nudibr. Moll., p. 44, fig.

Occasionally under stones on most rocky shores (s.P.): below the

Lab. (E.J.A., W.G., S.P.): Millbay Ch. (w.I.B., R.A.T.): Drake's I. (E.J.A.):

Cattewater; Rum B.; Bovisand Cove (w.G.): Cawsand B. (T.v.H.):

Rame-Eddystone Gds.; Yealm R., occasionally (R.A.T.).

Breeding: Feb.; May; July; Sept. (w.G.).

Goniodoris nodosa (Montagu): J. Alder \& Hancock, Monogr. Brit. Nubibr. Moll., p. 44, fig.

Plentiful on most shore stations and in dredgings from the Sound (w.I.B., s.P.).

Breeding: Jan.-Apr. (w.G., R.A.T.): May; Sept.; Dec. (w.G.). Adults, with spawn, in large numbers on the shores of the Sound from Feb. to May; small specimens abundant in dredgings in June, July, and Aug.; probably an annual migrating to the shores to spawn (W.G.). 
[Nudibranchiata-contd.: Pulmonata: Scaphopoda: Pelecypoda]

Idalina (Idaliella) ASPERSA (Alder \& Hancock): J. Alder \& Hancock, Monogr. Brit. Nudibr. Moll., p. 46, fig. (as Idalia).

Yealm Est. ${ }^{1}$; off Penlee Pt. ${ }^{1}$ (w.G.).

Ancula cristata (Alder): J. Alder \& Hancock, Monogr. Brit. Nudibr. Moll., p. 45, fig.

Rocks below the Lab. (E.J.A.): near the Ladies' Bathing Pool (w.G., T.V.H.): on the piles at Millbay Dock (R.A.T.): Drake's I. (w.G., W.I.B., R.A.T.) : Cawsand B. (w.G.): Batten B., amongst corallines, etc. (W.I.B.): Jennycliff B. (R.A.T.).

\section{PULMONATA.}

Otinidæ.

Otina otis (Turton): J. G. Jeffreys, Brit. Conch., vol. v, p. 110, fig.

Common in empty barnacle shells near high-water mark on the rocks under West Hoe and below Laboratory (R.A.T.): very abundant near high-water mark in rock crevices, etc., gregarious within limited areas; Drake's I.; cave under Ram's Cliff Pt.; etc. (s.P.).

\section{Auriculidæ.}

Leuconia bidentata (Montagu): J. G. Jeffreys, Brit. Conch., vol. v, p. 104, fig. (as Melampus).

Rocks under West Hoe, in empty barnacle shells (R.A.T.): common in crevices of rocks near high-water mark; Jennycliff B.; Drake's I.; etc. (S.P.).

\section{SCAPHOPODA.}

Dentalium entalis, Linnæus: J. G. Jeffreys, Brit. Conch., vol. iii, p. 191, fig.

Not uncommon on the fine sand grounds, $15-30$ fms. (R.A.T., s.P.): Eddystone Gds., on all fine sand grounds except the 'Outer' trawling ground, and occasionally on gravel (E.J.A.).

\section{PELECYPODA.}

\section{Nuculidæ.}

Nucula nitida, G. B. Sowerby: E. Forbes \& Hanley, Hist. Brit. Moll., vol. ii, p. 218 , fig.

Jennycliff B., Cawsand B. ; in muddy sand (R.A.T.): Eddystone Gds., in the fine sand of the 'Outer' trawling ground (E.J.A.).

Nucula nucleus (Linnæus): E. Forbes \& Hanley, Hist. Brit. Moll., vol. ii, p. 215, fig.

Common on muddy gravel, 10-30 fms. (S.P.): Millbay shellgravel; Cawsand B.; Mewstone Gds. (R.A.T.): Eddystone Gds. (E.J.A.).

\section{Anomiidæ.}

Anomia ephippium, Linnæus: J. G. Jeffreys, Brit. Conch., vol. ii, p. 30 , fig.

More or less common everywhere, L.W.-30 fms., on stones, shells, rocks, etc. (R.A.T.): Eddystone Gds., wherever suitable attachment can be found, least frequent on the fine sand grounds (E.J.A.). 
[Pelecypoda-contd.]

Anomia patelliformis, Linnæus: J. G. Jeffreys, Brit. Conch., vol ii, p. 34, fig.

Eddystone Gds., wherever suitable attachment is to be found, least common on the fine sand grounds (E.J.A.).

Arcidæ.

Glyctmeris glycimeris (Linnæus): J. G. Jeffreys, Brit. Conch., vol. ii, p. 166, fig. (as Pectunculus).

Common in sandy and muddy gravel, 7-30 fms. (s.P.): moderately common on Mewstone Ledge in the patches of gravel between the rocks and occasionally from the other grounds round the Mewstone; Queen's Gd.; Cawsand B.; Rame-Eddystone Gds. (R.A.T.): Eddystone Gds. (E.J.A.).

Limopsis aurita (Brocchi): J. G. Jeffreys, Brit. Conch., vol. ii, p. 161, fig.

Several dead shells (s.P.).

Barbatia lactea (Linnæus): J. G. Jeffieys, Brit. Conch., vol. ii, p. 177, fig. (as Arca).

Occasionally between tide-marks, among stones, etc., and in 15-30 fms., particularly on muddy gravel (s.P.): Queen's Gd. (R.A.T., S.P.): Reny Rks., occasionally (R.A.T.).

Arca tetragona, Poli : J. G. Jeffreys, Brit. Conch., vol. ii, p. 180, fig.

Occasionally on all rocky shores, in crevices and among stones, L.W.-30 fms. (s.P.): not uncommon in rock crevices at Drake's I. and Rum B. ; occasionally from the Bridge and Millbay Ch. (R.A.T.).

\section{Mytilidæ.}

Mrtilus edulis, Linnæus: J. G. Jeffreys, Brit. Conch., vol. ii, p. 104, fig.

Very common on the coal-hulks moored in the Cattewater and on the piles of the Promenade Pier; young specs. are common between tide-marks on the rocks below the Hoe and occasionally from Drake's I. and Asia Sh. (R.A.T.): Yealm Sand-bank (S.P.).

Volsella barbata (Linnæus): J. G. Jeffreys, Brit. Conch., vol. ii, p. 114, fig. (as Mytilus).

Occasionally between tide-marks, under boulders (S.P.): Asia Sh. (R.A.T.).

Volsella phaseolina (Philippi): J. G. Jeffreys, Brit. Conch., vol. ii, p. 118, fig. (as Mytilus).

Not uncommon on the outside grounds in $15-30 \mathrm{fms}$; occasionally between tide-marks on the Breakwater and Drake's I.; Millbay Pit (R.A.T.).

Modiolaria marmorata (Forbes): J. G. Jeffreys, Brit. Conch., vol, ii, p. 122 , fig.

More or less common, buried in the tests of Tuuicates; Millbay Pit and Ch.; Mewstone Ledge; Yealm R.; etc. (R.A.T., S.P.).

\section{Pteriidæ.}

Pteria hirundo (Linnæus) : E. Forbes \& Hanley, Hist. Brit. Moll., vol. ii, p. 251, fig. (as Avicula tarentina).

Very rare from the offing (R.A.T.). 
[Pelecypoda-contd.]

Pinna fragilis, Pennant: J. G. Jeffreys, Brit. Conch., vol. ii, p. 99, fig. (as $P$. rudis).

Small specs. are occasionally taken on gravel off Rame Hd., 20-30 fms.; dead shells are common (R.A.T.): Queen's Gd.; Stoke Pt. Gds., etc.; small specs. only (s.P.).

\section{Ostreidæ.}

Ostrea edulis, Limnæus: J. G. Jeffreys, Brit. Conch., vol. ii, p. 38, fig. Millbay Ch.; Queen's Gd.; Mewstone Ledge; Yealm R.; stray specs. occasionally dredged (R.A.T.): inside the Bridge (S.P. ${ }^{1}$ ). Extensive oyster-beds are cultivated in the Tamar R. at Saltash, and in the Yealm R. (E.J.A.).

\section{Pectinidæ.}

Pecten maximus (Limnæus): $J$. G. Jeffieys, Brit. Conch., vol. ii, p. 73, fig.

Mewstone 'Echinoderm' Gd., not uncommon; Rame-Eddystone Gds., etc. (R.A.T.): Eddystone Gds., moderately abundant on the various gravel grounds (E.J.A., S.P.): Mewstone Ledge; Asia Sh.; Yealm Sand-bank, between tide-marks ${ }^{1}$ (R.A.T.).

Pecten (Hinnites) pusio (Linnæus): J. G. Jeffreys, Brit. Conch., vol. ii, p. 51, fig.

Not uncommon on all rocky shores, among stones, inside dead shells, etc., L.W.-30 fms. (S.P.): Drake's I. ; Asia Sh.; Queen's Gd.; the Breakwater; etc. (R.A.T., S.P.).

Pecten (Chlamys) varius (Linnæus) : J. G. Jeffreys, Brit. Conch., vol. ii, p. 53, fig.

The Breakwater, not uncommon at low tide; occasional specs. from Drake's I., Asia Sh., Queen's Gd., Rum B., Yealm R. (R.A.T.).

Pecten (AEquipecten) opercularis (Linnæus): J. G. Jeffreys, Brit. Conch., vol. ii, p. 59, fig.

More or less common everywhere in 15-30 fms., especially on the Mewstone 'Echinoderm' Gd. and about $5 \mathrm{~m}$. S.W. of Penlee Pt. ; occasionally from Queen's Gd. and Asia Sh.; the Breakwater, single small spec. between tide-marks (R.A.T.): Eddystone Gds., more or less abundant on all the grounds, often in beds which, while of limited extent, contain very numerous individuals (E.J.A., S.P.).

Breeding: June-Aug. (S.P.).

Pecten (Palliolum) tigerinus (Müller): E. Forbes \& Hanley, Hist. Brit. Moll., vol. ii, p. 285, fig.

Not uncommon on coarse muddy or sandy gravel; Stoke Pt. Gds.; Rame-Eddystone Gds.; etc. (s.P.): Mewstone 'Echinoderm' Gd. (R.A.T., S.P.) : Eddystone Gds., not abundant, for the most part on coarse ground (E.J.A.): the Breakwater, single spec. between tide-marks (R.A.T.).

\section{Limidæ.}

Lima hians (Gmelin): J. G. Jeffreys, Brit. Conch., vol. ii, p. 87, fig.

Abundant at extreme low tide among a small patch of stones with muddy gravel on the N. side of the Breakwater ${ }^{1}$; Reny Rks. ${ }^{1}$ (R.A.T.): Drake's I. (s.P. $\left.{ }^{1}\right)$. 
[Pelecypoda - contd.]

Lima Loscombi, G. B. Sowerby: J. G. Jeffreys, Brit. Conch., vol. ii, p. 85 , fig.

Not uncommon on the coarse grounds W. of the Eddystone (E.J.A., S.P.) : Rame-Eddystone Gds. (s.P.): Mewstone Gds. (R.A.T., S.P.): Stoke Pt. Gds. (W.G., S.P.).

Lima subauriculata (Montagu): J. G. Jeffreys, Brit. Conch., vol. ii, p. 82, fig.

Dead shells only (R.A.T.).

\section{Astartidæ.}

Astarte sulcata (da Costa): E. Forbes \& Hanley, Hist. Brit. Moll., vol. i, p. 452 , fig.

Not uncommon, 15-30 fms., generally on muddy or sandy gravel (R.A.T., S.P.).

\section{Cyprinidæ.}

Cyprina islandica (Linnæus): E. Forbes \& Hanley, Hist. Brit. Moll., vol. i, p. 441, fig.

Occasionally, 15-30 fms.; dead shells moderately common (R.A.T.).

\section{Lucinidæ.}

Lucina borealis (Linnæus): J. G. Jeffreys, Brit. Conch., vol. ii, p. 242, fig.

Occasionally on the Zostera bed N.E. of Drake's I. and on the Yealm Sand-bank; dead shells are very common in Cawsand B. (R.A.T.).

Lucina spinifera (Montagu): E. Forbes \& Hanley, Hist. Brit. Moll., vol. ii, p. 49, fig.

Rame-Eddystone Gds. (S.P. $\left.{ }^{1}\right)$ : dead shells not uncommon (R.A.T.).

Thyasira flexuosa (Montagu): J. G. Jeffreys, Brit. Conch., vol. ii, p. 247, fig. (as Axinus).

Yealm Sand-bank, occasionally; dead shells are very common in Cawsand B. (R.A.T.).

Montacuta substriata (Montagu): J. G. Jeffreys, Brit. Conch., vol. ii, p. 205, fig.

Common attached to the anal spines of Spatangus purpureus (R.A.T., S.P.).

Tellimya ferruginosa (Montagu): E. Forbes \& Hanley, Hist. Brit. Moll., vol. ii, p. 72, fig. (as Montacuta).

Yealm Sand-bank, common commensal with Echinocardium cordatum (R.A.T.).

Diplodonta rotundata (Montagu) : J. G. Jeffreys, Brit. Conch., vol. ii, p. 254 , fig.

Dead shells only (R.A.T.).

\section{Leptonidæ.}

Kellia suborbicularis (Montagu): E. Forbes \& Hanley, Hist. Brit. Moll., vol. ii, p. 87, fig.

Not uncommon on all rocky shores, L.W.-30 fms., in crevices, inside dead shells, etc., particularly where there is a thin layer of silt; gregarious (S.P.): Drake's I.; Asia Sh.; Queen's Gd.; Rum B. ; 
[ Pelecypoda-contd.]

Millbay Ch., in Saxicava borings; etc. (R.A.T., S.P.): Stoke Pt. Gds., in Pholadidea crypts, etc. (s.P.): Eddystone Gds.; generally in fine mud inside dead shells (E.J.A., S.P.).

Las p. 94, fig. (as Poronia).

Abundant between tide-marks on all rocky shores, in crevices, etc. (s.P.): rocks below the Hoe, very common among barnacles and the roots of Fucus, etc. (R.A.T.).

Lepton squamosum (Montagu): E. Forbes \& Hanley, Hist. Brit. Moll., vol. ii, p. 98 , fig.

Dead shells only (R.A.T.).

\section{Scrobiculariidæ.}

Scrobicularia plana (da Costa): E. Forbes \& Hanley, Hist. Brit. Moll., vol. i, p. 326, fig. (as S. piperata).

St. John's Lake, Hamoaze, common in fine tenacious mud (R.A.T.).

\section{Tellinidæ.}

Tellina crassa (Gmelin): E. Forbes \& Hanley, Hist. Brit. Moll., vol. i, p. 288 , fig.

Drake's I., occasionally between tide-marks (R.A.T.): Mewstone Gds., common; Rame-Eddystone Gds. (R.A.T., s.P.): Stoke Pt. Gds. (s.P.): Eddystone Gds. (E.J.A., S.P.): Yealm R., occasionally on coarse sand between tide-marks (R.A.T.).

Tellina donacina, Linnæus: E. Forbes \& Hanley, Hist. Brit. Moll., vol. i, p. 292, fig.

Yealm R., occasionally in coarse sand between tide-marks (R.A.T.).

Tellina fabula, Gronovius: E. Forbes \& Hanley, Hist. Brit. Moll., vol. i, p. 302, fig.

Not uncommon in the muddy sand of the Zostera beds; Cawsand B.; Batten B.; Jennycliff B.; etc. (s.P.).

Tellina squalida, Pulteney: E. Forbes \& Hanley, Hist. Brit. Moll., vol. i, p. 298, fig. (as T. incarnata).

Dead shells only (R.A.T.).

Macoma balthica (Linnæus): E. Forbes \& Hanley, Hist. Brit. Moll., vol. i, p. 304, fig. (as Tellina solidula).

Saltash (E.J.A. ${ }^{1}$ ).

\section{Donacidæ.}

Donax variegatus (Gmelin): E. Forbes \& Hanley, Hist. Brit. Moll, vol. i, p. 336, fig. (as D. politus).

Dead shells only (R.A T.).

Donax vittatus (da Costa): E. Forbes \& Hanley, Hist. Brit. Moll., vol. i, p. 332, fig. (as D. anatinus).

Whitsand B., occasional specimens (R.A.T.).

\section{Mactridæ.}

Mactra stultorum, Linnæus: J. G. Jeffieys, Hist. Brit. Moll., vol. ii, p. 422 , fig.

Dead shells only; these are common in West Ch. and Whitsand B. (R.A.T.). 
[Pelecypoda-contd.]

Spisula elliptica (Brown): E. Forbes \& Hanley, Hist. Brit. Moll., vol. i, p. 356, fig. (as Mactra).

West Entrance, abundant; Cawsand B., moderately common (s.P.).

Spisula solida (Linnæus): E'. Forbes \& Hanley, Hist. Brit. Moll., vol. i, p. 351, fig. (as Mactra).

Drake's I., common on sand-bank at E. end (s.P. \& A.J.s.): Yealm Sand-bank (R.A.T.).

Lutraria elliptica, Lamarek: J. G. Jeffreys, Brit. Conch., vol. ii, p. 428 , fig.

Drake's I., abundant in a small sandy patch on the Zostera bed on the N.E. side (s.P.): on the shore S. of Batten Castle and Yealm Sand-bank, occasionally (R.A.T.).

Gonads ripe: Mar.-Apr. (s.P.): May (R.A.T.).

\section{Veneridæ.}

Lucinopsis undata (Pennant): E. Forbes \& Hanley, Hist. Brit. Moll., vol. i, p. 435 , fig.

Dead shells only (R.A.T.).

Dosinia exoleta (Linnæus): E. Forbes \& Hanley, Hist. Brit. Moll., vol. i, p. 428, fig. (as Artemis).

Occasionally from gravel S. of Drake's I. and in Millbay Ch.; Yealm Sand-bank, not uncommon (R.A.T.) : Mewstone Gds.; Stoke Pt. Gds.; Rame-Eddystone Gds.; etc. (s.P.): Eddystone Gds. (E.J.A., S.P.).

Meretrix chione (Linnæus): E. Forbes \& Hanley, Hist. Brit. Moll., vol. i., p. 396, fig. (as Cytherea).

Dead shells only (R.A.T.).

Venus (Clausinella) fasciata (da Costa): E. Forbes \& Hanley, Hist. Brit. Moll., vol. i, p. 415, fig.

Common on coarse gravel, particularly on muddy or sandy gravel, 6-30 fms.; Queen's Gd.; Mewstone Gds.; Stoke Pt. Gds.; Rame-Eddystone Gds.; Eddystone Gds. (s.P.): Yealm Sand-bank, occasionally between tide-marks (R.A.T.).

Venus (Ventricola) casina, Linnæus: E. Forbes \& Hanley, Hist. Brit. Moll., vol. i, p. 405, fig.

Occasionally on gravel grounds, $10-30$ fms. (s.P.).

Venus (Ventricola) verrucosa, Linnæus: E. Forbes \& Hanley, Hist. Brit. Moll., vol. i, p. 401, fig.

Occasionally on mixed gravel grounds, 15-30 fms.; Rame-Eddystone Gds.; Mewstone Gds.; etc. (s.P.): Millbay Ch.; Yealm Sand-bank (R.A.T.).

Venus (Timoclea) ovata, Pennant: E. Forbes \& Hanley, Hist. Brit. Moll., vol. i, p. 419, fig.

Moderately common in the Sound on muddy sand and gravel, and occasionally outside in 15-30 fms. (R.A.T.).

Venus (Chamelæa) gallina, Linnæus: E. Forbes \& Hanley, Hist. Brit. Moll., vol. i, p. 408, fig. (as V. striatula).

Cawsand B., moderately common in sand; occasionally on the 
[Pelecypoda-contd.]

outside grounds, 15-30 fms.; Yealm Sand-bank, between tide-marks (R.A.T.).

Tapes aureus (Gmelin): E. Forbes \& Hanley, Hist. Brit. Moll., vol. i, p. 392, fig.

Dead shells only (R.A.T.).

Tapes decussatus (Linnæus): E. Forbes \& Hanley, Hist. Brit. Moll., vol. i, p. 379 , fig.

Laira (A.J.S.): Yealm Sand-bank, not uncommon (s.P.).

Tapes pullastra (Montagu): E. Forbes \& Hanley, Hist. Brit. Moll., vol. i, p. 382, fig.

Occasionally between tide-marks and in dredgings from the Sound; the variety "perforans" is moderately common between tide-marks where the rocks are bored by Saxicava; Yealm Sandbank, common (R.A.T.).

Tapes virgineus (Linnæus): E. Forbes \& Hanley, Hist. Brit. Moll., vol. i, p. 388 , fig.

Common in gravel in Millbay Ch.; occasional specs. from Drake's I., Jennycliff B., the Breakwater, Reny Rks., and from the outside grounds in $15-30$ fms. (R.A.T.).

Gouldia minima (Montagu): E. Forbes \& Hanley, Hist. Brit. Moll., vol. i, p. 446, fig. (as Circe).

Not uncommon on fine and medium gravel, 5-30 fms. ; Queen's Gd., occasionally; Mewstone Gds.; Stoke Pt. Gds. (s.P.): Eddystone Gds. (E.J.A., S.P.).

\section{Cardiidæ.}

Cardium echinatum, Linnæus: E. Forbes \& Hanley, Hist. Brit. Moll., vol. ii, p. 7, fig.

Living specs. are seldom obtained, 15-30 fms., although dead shells are moderately common; Cawsand B., single spec. (R.A.T.): Eddystone Gds., fresh dead shells are a characteristic feature of the fine sand of the 'Inner' and 'Outer' Trawling Gds. (E.J.A.).

Cardium edule, Limnæus: E. Forbes \& Hanley, Hist. Brit. Moll., vol. ii, p. 15 , fig.

Hamoaze, abundant in places; occasional specs. from Laira, Drake's I. and Rum B.; Yealm R., abundant just below Steer Pt. (A.J.S.).

Cardium (Lavicardium) norvegicum (Spengler): E. Forbes \& Hanley, Hist. Brit. Moll., vol. ii, p. 35, fig.

Common on gravel, 15-30 fms.; West Ch. and Queen's Gd., occasionally; Mewstone Ledge, moderately common in the patches of gravel between the rocks; Mewstone 'Amphioxus' Gd.; RameEddystone Gds. ; etc. (R.A.T., S.P.): Eddystone Gd. (E.J.A., S.P.).

\section{Garidæ.}

Gari costulata, Turton: J. G. Jeffreys, Brit. Conch., vol. ii, p. 394, fig. (as Psammobia).

Occasionally with G. tellinella; Rame-Eddystone Gds.; Eddystone Gds. ; etc. (s.P.). 
[Pelecypoda-contd.]

Gari ferromnsis (Chemnitz): J. G. Jeffireys, Brit. Couch., vol. ii, p. 396, fig. (as Psammobia).

Small specs. not uncommon in Cawsand B., and dead shells are moderately common in $15-30$ fms. (R.A.T.).

Gari tellinella, Lamarck: J. G. Jeffreys, Brit. Conch., vol. ii, p. 392, fig. (as Psammobia).

Millbay Ch.; Mewstone Gds., common in gravel and coarse sand (R.A.T.): Stoke Pt. Gds.; Rame-Eddystone Gds. ; etc. (s.P.).

Gari (Psammocola) depressa, Pennant: J. G. Jeffieys, Brit. Conch., vol. ii, p. 398, fig. (as Psammobia vespertina).

Yealm R., not uncommon in coarse sand between tide-marks (R.A.T.).

\section{Myidæ.}

Mya truncata, Linnæus: E. Forbes \& Hanley, Hist. Brit. Moll., vol. i, p. 163 , fig.

Single spec., Yealm Sand-bank (A.J.s.).

Corbula gibba (Olivi): E. Forbes \& Hanley, Hist. Brit. Moll., vol. i, p. 180, fig. (as C. nucleus and C. rosea).

Jennycliff B.; Cawsand B.; occasionally (R.A.T., S.P.): Mewstone Gds.; Stoke Pt. Gds.; Rame-Eddystone Gds., not uncommon on muddy gravel (s.P.).

\section{Solenidæ.}

Solecurtus antiquatus (Pulteney) : J. G. Jeffreys, Brit. Conch., vol. iii, p. 6 , fig.

Jennycliff B., single spec.; $4 \mathrm{~m}$.W. by S. of Rame Hd., single spec.; dead shells are not uncommon 2-4 m. S.W. of Rame Hd. (R.A.T.).

Solecurtus scopula (Turton): J. G. Jeffreys, Brit. Conch., vol. iii, p. 3, fig. (as $S$. candidus).

Eddystone Gds., single spec. (R.A.T.).

Cultellus pellucidus (Pennant): J. G. Jeffieys, Brit. Conch., vol. iii, p. 14, fig. (as Solen).

Jennycliff B.; Cawsand B.; common (R.A.T., S.P.) : Mallard Sh., in sandy mud (s.P.).

Ensis Ensis (Linnæus): J. G. Jeffreys, Brit. Conch., vol. iii, p. 16, fig. (as Solen).

Yealm Sand-bank, very common; Drake's I., not uncommon in sand at the N.E. corner; sandy patch S. of Batten Castle; Cawsand B.; etc. (R.A.T.).

Ensis siliqua (Linnæus): J. G. Jeffreys, Brit. Conch., vol. iii, p. 18, fig. (as Solen).

Dead shells only (R.A.T.).

Solen vagina, Linnæus: J. G. Jeffireys, Brit. Conch., vol. iii, p. 20, fig.

Drake's I., occasionally on muddy sand N. of the Island (s.P.): Yealm Sand-bank (R.A.T.).

\section{Saxicavidæ.}

Saxicava arctica (Linnæus): E. Forbes \& Hanley, Hist. Brit. Moll., vol. i, p. 141, fig.

Mewstone Gds.; Rame-Eddystone Gds. (R.A.T.): Eddystone Gds., on all grounds, attached to shells, the roots of Hydroids, etc. (E.J.A.). 
[Pelecypoda-contd.]

Saxicava Rugosa (Linnæus): E. Forbes \& Hanley, Hist. Brit. Moll., vol i., p. 146 , fig.

Every where boring in limestone, etc., L.W.-20 fms. (R.A.T.).

\section{Gastrochænidæ.}

Gastrochena dubia (Pennant): E. Forbes \& Hanley, Hist. Brit. Moll., vol. i, p. 132, fig. (as G. modiolina).

The Breakwater, boring in limestone; Asia Sh. ; Millbay Ch. and Pit; Yealm R., single spec. boring in an oyster shell (R.A.T.).

\section{Pholadidæ.}

Pholas dactylus, Linnæus: E. Forbes \& Hanley, Hist. Brit. Moll., vol. i, p. 108, fig.

Rum B., common in particular patches of shale rock (R.A.T., A.J.S.).

Barnea Parva (Pennant): E. Forbes \& Hanley, Hist. Brit. Moll., vol. i, p. 111, fig. (as Pholas).

Rum B., common (R.A.T.).

Pholadidea loscombiana, Goodall: E. Forbes \& Hanley, Hist. Brit. Moll., vol. i, p. 123, fig. (as P. papyracea).

Rum B., common at low tide (R.A.T.): abundant in the red rock of the Mewstone Ledge, Stoke Pt., etc., 10-20 fms. (s.P.).

\section{Teredinidæ.}

Teredo navalis, Linnæus: E. Forbes \& Hanley, Hist. Brit. Moll., vol. i, p. 74 , fig.

Not uncommon in drift and submerged wood (R.A.T.).

Breeding: July (w.G.).

\section{Pandoridæ.}

Pandora in equivalvis (Linnæus): E. Forbes \& Hanley; Hist. Brit. Moll., vol. i, p. 207 (as P. rostrata), p. 210 (as P. obtusa), fig.

Cawsand B., occasionally (R.A.T.): Rame-Eddystone Gds. (R.A.T., S.P.) : Eddystone Gds. (E.J.A., S.P.): Stoke Pt. Gds. (s.P.).

\section{Lyonsiidæ.}

Lyonsia norvegica (Chemnitz): E. Forbes \& Hanley, Hist. Brit. Moll., vol. i, p. 214 , fig.

Cawsand B., occasionally (R.A.T.): single spec. off Stoke Pt. (E.J.A.).

\section{Anatinidæ.}

Cochlodesma pretenue (Pulteney): E. Forbes \& Hanley, Hist. Brit. Moll., vol. i, p. 235, fig.

Dead shells only (R.A.T.).

Thracia fragilis, Pennant: E. Forbes \& Hanley, Hist. Brit. Moll., vol. i, p. 221 fig. (as T. phaseolina).

Single spec., S. of Batten Castle, in sand (R.A.T.).

Thracia pubescens (Pulteney): J. G. Jeffreys, Brit. Conch., vol. iii, p. 38 , fig.

Dead shells only (R.A.T.). 


\section{CEPHALOPODA.}

\section{Ommastrephidæ.}

Todaropsis Eblane (Ball): W. E. Hoyle, Journ. Mar. Biol. Assoc., ser. 2, vol. ii, p. 189.

Plymouth neighbourhood, single spec. (J.T.c. \& w.E.H.).

\section{Loliginidæ.}

Loligo Forbesi, Steenstrup: W. E. Hoyle, Proc. Phys. Soc. Edinburgh, vol. viii, p. 459 .

The Sound, occasionally (W.G.): practically absent, Jan.-June 1895 (E.J.A.) : Tamar R., below Saltash Bridge; Lynher R.; small specs. common, July 1897 (E.W.L.H.): 'Inner' trawling grounds, large spec. Aug. 1897 (w.I.B.).

Breeding: Apr.-Sept. (w.G.).

Loligo Marmore, Vérany: A. M. Norman, Ann. Mag. Nat. Hist., ser. 6, vol. v, p. 481.

Off the Draystone, single spec. (w.G.).

Loligo media (Linnæus): A. M. Norman, Ann. Mag. Nat. Hist., ser. 6, vol. v, p. 482.

Cawsand B.; Whitsand B.; etc. (w.G.): Jennycliff B. (R.A.T.): St. John's Lake, Hamoaze, Aug. 1898 (E.w.L.H.).

Breeding: Apr.-June (w.G.). Late embryos in Oct. (w.G.).

\section{Sepiidæ.}

SePia Elegans, d'Orbigny: G. Jatta, Fauna Flora Neapel, vol. xxiii, p. 160 , fig.

Not uncommon on the trawling grounds (E.w.L.H., W.I.B., S.P.).

Sepia officinalis, Linnæus: G. Jatta, Fauna Flora Neapel, vol. xxiii, p. 149, fig.

Comes into the bays and estuaries to spawn during July and Aug. (w.I.B.): Mewstone Gds.; Rame-Eddystone Gds.; etc., occasionally (R.A.T.) : very scarce during 1904 (A.J.S.).

Breeding: July-Sept. (R.A.T.). Hatching: Oct. (R.A.T.).

\section{Sepiolidæ.}

Sepiola Atlantica, d'Orbigny: A. M. Norman, Ann. Mag. Nat. Hist., ser. 6 , vol. v, p. 473 .

Common in Cawsand B., Jennycliff B., and Whitsand B.; occasionally on the outside grounds in 15-30 fms. (R.A.T., S.P.): the Cattewater, common (R.A.T.): Tamar R., common just below Saltash Bridge; Downderry, common in sandy pools; Yealm R. (w.I.B.).

Sepiola scandica, Steenstrup: A. M. Norman, Ann. Mag. Nat. Hist., ser. 6 , vol. v, p. 472 .

The Sound, single spec. Nov. 1887 (w.н.): Mewstone Gds., single spec. Oct. 1899 (w.I.B.).

Rossia macrosoma (delle Chiaji): A. M. Norman, Ann. Mag. Nat. Hist., ser. 6 , vol. v, p. 469 .

Two specs., autumn 1892 (w.G.): occasionally on the Trawling Gds. (A.J.S.). 


\section{Polypodidæ.}

Polypus vulgaris (Lamarck): J. G. Jeffreys, Brit. Conch., vol. v, p. 144, fig. (as Octopus).

On the shore and from lobster pots; generally only a few specimens are obtained during the summer months, but it varies greatly in abundance in different years (E.J.A.) : exceptionally plentiful * in 1900 (W.G.).

Moschites cirrosa (Lamarck): J. G. Jeffreys, Brit. Conch., vol. v, p. 146 (as Eledone), fig.

Frequently met with on the trawling grounds (E.J.A.): commonly caught in lobster pots (A.J.S.).

\section{TUNICATA.}

\section{Molgulidæ.}

Molgula oculata, Forbes: $H$. de Lacaze-Duthiers, Arch. Zool. expt., ser. 1, vol. vi, p. 516, fig. (as Anurella).

Mewstone Ledge (т.V.H.): not uncommon in clean fine gravel off the Mewstone (R.A.T.): Eddystone Gds. (E.J.A.).

Molgula simplex, Alder \& Hancock: $H$. de Lacaze-Duthiers, Arch. Zool. expt., ser. 1, vol, vi, p. 542, fig. (as Anurella).

Eddystone Gds., on Choetopterus tubes, not uncommon (E.J.A.).

\section{Cynthiidæ.}

Forbesella tessellata (Forbes): E. Forbes \& Hanley, Hist. Brit. Moll., vol. i, p. 39, fig. (as Cynthia limacina); W. A. Herdman, Journ. Linn. Soc., Zool., vol. xxiii, p. 578.

Rame-Eddystone Gds. (R.A.T.): Eddystone Gds., single spec. on fine gravel (E.J.A.).

Styelopsis grossularia, v. Beneden: $R$. Hartmeyer, Meeresf. Bergen [Bergens Mus. Publ.], pt. i, p. 46.

Abundant on rocks between tide-marks, Jennycliff B., Rum B., Mt. Edgeumbe; occasionally in dredgings from Millbay Ch., Queen's Gd., etc. (R.A.T.): Eddystone Gds., the small squat var. not uncommon on shells, particularly Pecten shells, on the gravels W. of the Eddystone (E.J.A.).

Breeding: May; Oct. (w.G.).

Polycarpa pomaria (Savigny): $R$. Hartmeyer, Fauna Arctica, vol. iii, p. 229.

Common at times in the Cattewater, but probably from trawl refuse; very plentiful on one occasion 4 m. W.N.W. of the Eddystone (R.A.T.): Eddystone Gds. (E.J.A.): Mewstone Ledge (A.J.S.).

\section{Ascidiidæ.}

Corella larvafformis, Hancock: W. A. Herdman, Journ. Linn. Soc., Zool., vol, xxiii, p. 588 .

Off the Eddystone (w.G.).

* Journ. Mar. Biol. Assoc., ser. 2, vol, ii, pp. 260-73. 
[Tunicata-contd.]

Corella parallelogramma (O. F. Müller): $R$. Hartmeyer, Meeresf. Bergen [Bergens Mus. Publ.], pt. i, p. 42, fig.

Duke Rk., single spec. on small stone; $2 \mathrm{~m}$. S. of Mewstone, single spec. on stone (w.G.).

Phallusia mamllata, Cuvier: $M$. P. A. Traustedt, Mitth. Zool.-Stat. Neapel, vol. iv, p. 456 , fig.

Moderately common in dredgings from the Yealm R., Mewstone Ledge; and occasionally from the Duke Rk., Cattewater, and West Ch. Not uncommon washed up on the Yealm Sand-bank between tide-marks (R.A.T.).

Ascidiella aspersa (O. F. Müller): $W$. A. Herdman, Journ. Linn. Soc., Zool., vol. xv, p. 281, fig. (as Ascidia).

Common on the piles of the Promenade Pier, and in Millbay Dock (R.A.T.) : Cattewater (T.V.H.): occasionally between tide-marks, Rum B. (R.A.T.): Yealm R. (R.A.T.): Yealm Sand-bank (A.J.S., R.A.T.). Breeding: Apr. (w.G.): June-July (R.A.T.).

Ascidiella scabra, O. F. Müller: $W$. A. Herdman, Journ. Linn. Soc., Zool., vol. xv, p. 284, fig. (as Ascidia).

Duke Rk.; Yealm R. (w.G.): Eddystone Gds., generally present, but particularly abundant where Sertularella Gayi is plentiful, growing at the base of the stem of the Hydroid (E.J.A., S.P.) : Mewstone Gds. (A.J.S., R.A.T., S.P.): Rame-Eddystone Gds. (R.A.T., S.P.): Stoke Pt. Gds. (S.P.).

Ascidia venosa, O. F. Müller: R. Hartmeyer, Meeresf. Bergen [Bergens Mus. Publ.], pt. i, p. 36, fig.

Eddystone Gds., occasional specs. (E.J.A.).

Ascidia depressa, Alder: W. Garstang, Journ. Mar. Biol. Assoc., ser. 2, vol. ii, p. 125 , fig.

Not uncommon on the Bolt Head shell gravel attached to stones (E.J.A.).

Ascidia mentula, O. F. Müller: W. Garstang, Journ. Mar. Biol. Assoc., ser. 2, vol. ii, p. 130 , fig.

Reny Rks., single spec., var. depressa (w.G., R.A.T.): Millbay Ch.; Mewstone Gds. (E.J.A.).

Ciona intestinalis (Linnæus): R. Hartmeyer, Fauna Aretica, vol. iii, p. 297 , fig.

Until 1901 this Ascidian was comparatively rare, having only been recorded occasionally from Millbay Ch., the Cattewater, Yealm R., Mewstone Ledge, and the Eddystone Gds., and the specimens were never of greater length than 4-5 inches. In that year the species became for a time very abundant in Millbay Docks, completely covering the piles and rafts, and many of the specimens from the Inner Dock were of extremely large size, measuring as much as a foot in length (E.J.A.): Stoke Pt. Gds., small specimens fairly common (s.P.).

Breeding: June-July (R.A.T.): Aug.-Sept. (w.G.). 
[ Tunicata_contd.]

\section{Clavelinidæ.}

Diazona violacea, Savigny: $W$. Garstang, Journ. Mar. Biol. Assoc., ser. 2, vol. ii, p. 63 .

Eddystone Gds. (W.G., R.A.T. ${ }^{1}$ ): Stoke Pt. Gds. (w.G.).

Perophora banyulensis, Lahille.

Duke Rk. (w.G.).

Perophora Listeri, Wiegmann: W. Garstang, Journ. Mar. Biol. Assoc., ser. 2, vol. ii, p. 58, fig.

Rocks under the Hoe, abundant (w.H.): Queen's Gd.; Millbay Pit; Asia Sh. (A.J.S.): Duke Rk.; Yealm Estuary (w.G.).

Pycnoclavella aurilucens: $W$. Garstang, Journ. Mar. Biol. Assoc., ser. 2 , vol. ii, p. 53, fig.

Mewstone Gds., attached to various objects from rough ground, $10-20 \mathrm{fms}$. ; once in the Sound, forming a thin growth on the stems of red weeds (w.G.): Mewstone Ledge, on Eunicella (R.A.T., S.P.).

Clatelina lepadiformis (O. F. Müller): $W$. Garstang, Journ. Mar. Biol. Assoc., ser. 2, vol. ii, p. 51.

Drake's I., occasionally at extreme low tide (w.G., R.A.T.): Queen's Gd. (R.A.T., S.P.) : Mt. Edgeumbe (w.G., T.v.H.): Duke Rk. (W.G., R.A.T.): Rum B.; Millbay Ch.; Mewstone Ledge; Wembury B. (т.v.H.): very rarely, in 10-15 fms. off the Mewstone and Penlee (W.G.).

Breeding: June (w.G.): July (R.A.T.).

\section{Distomidæ.}

Distaplia rosea, Della Valle: W. A. Herdman, Journ. Linn. Soc., Zool., vol. xxiii, p. 613 .

On stones, dead shells, etc., at Duke Rk. and elsewhere (w.G.).

Archidistoma AgGregatum: $W$. Garstang, Zool. Anz., vol. xiv, p. 422, fig.

Duke Rk., very abundant on stones; $2 \mathrm{~m}$. S. of Mewstone ${ }^{1}$ (w.G.).

Breeding: June (w.G.).

\section{Polyclinidæ.}

Aplidium zostericola: A. Giard, Arch. Zool, expt., ser. 1, vol. i, p. 636, fig.

Plymouth,attached to the stalks of Bowerbankia or Amathia (w.G.).

Amaroucium albicans (H. Milne-Edwards): W. A. Herdman, Journ. Linn. Soc., Zool., vol. xxiii, p. 623.

Off the Mewstone (w.G.).

Amaroudium Nordmanni (H. Milne-Edwards): A. Giard, Arch. Zool. expt., ser. 1, vol. i, p. 636 .

Church Reef, Wembury B. (w.G. ${ }^{1}$ ).

Breeding: June (w.G.).

Amaroucium punctum: $A$. Giard, Arch. Zool, expt., ser. 1, vol. ii, p. 495.

Single colony, $3 \mathrm{~m}$. S. of the Mewstone (w.G.).

Morchellium argus, Milne-Edwards: W. A. Herdman, Journ. Linn. Soc., Zool., vol. xxiii, p. 625 .

Common everywhere on rocks between tide-marks (R.A.T.): Millbay Ch. (т.v.H.): Millbay Docks, on wooden piles (w.G.): Duke Rk. (E.J.A., R.A.T.): Yealm R. (T.V.H., R.A.T.).

Breeding: Sept. (w.G.). 
[ Tunicata-contd.]

Morchellioides Alderi: W. A. Herdman, Proc. Lit. Phil. Soc. Liverpool, vol, xl, p. 291, fig.

Asia Sh., on roots of Laminaria and on stones and shells; Millbay Ch., on stones and shells; Bovisand B., on stones and shells (w.G.).

Fragarium elegans : A. Giard, Arch. Zool. expt., ser. 1, vol. i, p. 638, fig.

Duke Rk., on stones (w.G.).

Circinalium concrescens: A. Giard, Arch. Zool. expt., ser. 1, vol. i, p. 639 , fig.

Drake's I., on reef leading to the Bridge, on roots of Laminaria at extreme low-water; Picklecombe, E. of the Fort on roots of Laminaria; Devil's Pt., Stonehouse, under a stone, low-water, spring tide; Duke Rk., in crevices of stones; Wembury B., under overhanging rocks (W.G.).

\section{Botryllidæ.}

Botryllus violaceus, H. Milne-Edwards: A. Giard, Arch. Zool. expt., ser. 1, vol. i, p. 621, fig.

Common on rocks, under stones and on weed, at low water on all shores (w.G.).

Breeding: June-Aug. (w.G.).

Botrylloides rubrum, Milne-Edwards: A. Giard, Arch. Zool, expt., ser. 1, vol. i, p. 632, fig.

Under the Hoe, common under stones (w.G.).

Breeding: Aug.-Oct. (w.G.).

\section{Doliolidæ.}

Doliolum nationalis, Borgert*: A. Borgert, Ergebn. Plankton Expedn., vol. ii, pt. E. a. C., p. 21, fig.

Tow-nettings, Aug. and Sept., 1893, a considerable number (w.G.): very abundant during 1895 (т.V.H.): S. of the Eddystone, several specs. Nov. 1904 (L.H.G.).

\section{Salpidæ.}

Salpa fusiformis, Cuvier: W.A. Herdman, Rept. Voy. Challenger, vol. xxvii. (pt. lxxvi), p. 74, fig. (as $S$. runcinata-fusiformis).

Aggregated form at surface, N. of Eddystone, Aug., 1901 (R.A.T.).

Salpa mucronata, Forskal: $W$. A. Herdman, Rept. Voy. Challenger, vol. xxvii (pt. lxxvi), p. 79, fig. (as S. democratica-mucronata).

Large shoals visited the Sound in 1893, from the middle of June to the end of the first week in July (w.G.).

Breeding: Aug.-Oct. (w.G.).

\section{Appendiculariidæ.}

Oikopleura Dioica, Fol: H. Lohmann, Ergebn. Plankton Exped., vol. ii, pt. E. c., p. 76, fig.

Generally present in tow-nettings, occasionally common (R.A.T.).

Breeding: Mar.-Apr. (w.G.).

Fritillaria borealis: $H$. Lohmann, Ergebn. Plankton Exped., vol, ii, pt. E. c., p. 49, fig.

Plymouth; Mar., Oct., Nov., 1899 (P.T.c.): Eddystone, Aug., 1903 (L.H.G.)

\footnotetext{
* Previously recorded as $D$. Tritonis, Herdman.
} 